\title{
Graphen auf Siliziumcarbid: elektronische Eigenschaften und Ladungstransport
}

\author{
Dissertation \\ zur Erlangung des mathematisch-naturwissenschaftlichen Doktorgrades \\ "Doctor rerum naturalium" \\ der Georg-August-Universität zu Göttingen \\ im Promotionsprogramm ProPhys \\ der Georg-August University School of Science (GAUSS) \\ vorgelegt von \\ Thomas Christian Druga \\ aus Hameln
}

Göttingen, 2014 
1. Betreuungsausschuss

Prof. Dr. Rainer G. Ulbrich,

IV. Physikalisches Institut, Georg-August-Universität Göttingen

Prof.in Dr. Cynthia A. Volkert,

Institut für Materialphysik, Georg-August-Universität Göttingen

Prof. Dr. Christian Jooß,

Institut für Materialphysik, Georg-August-Universität Göttingen

Dr. Martin Wenderoth,

IV. Physikalisches Institut, Georg-August-Universität Göttingen

2. Mitglieder der Prüfungskommission

Referent: Prof. Dr. Rainer G. Ulbrich,

IV. Physikalisches Institut, Georg-August-Universität Göttingen

Korreferent: Prof. Dr. Hans Hofsäss,

II. Physikalisches Institut, Georg-August-Universität Göttingen

3. Weitere Mitglieder der Prüfungskommission

Prof. Dr. Cynthia A. Volkert,

Institut für Materialphysik, Georg-August-Universität Göttingen

Prof. Dr. Vasily Moshnyaga,

I. Physikalisches Institut, Georg-August-Universität Göttingen

Prof. Dr. Astrid Pundt,

Institut für Materialphysik, Georg-August-Universität Göttingen

Prof. Dr. Michael Seibt,

IV. Physikalisches Institut, Georg-August-Universität Göttingen

Tag der mündlichen Prüfung: 07.03.2014 




\section{Einleitung}

Um leistungsfähigere elektrische Bauelemente mit einer immer besseren Energieeffizienz zu erreichen, wird die Strategie der Miniaturisierung verwendet, wie sich an der zeitlichen Entwicklung der Gate-Länge von Transistoren nachvollziehen lässt. Diese liegt inzwischen bei ungefähr $20 \mathrm{~nm}$ und soll in den nächsten Jahren auf bis zu $10 \mathrm{~nm}$ reduziert werden [1]. Die herkömmliche Silizium-Technologie stößt dabei an ihre Grenzen und es wird nach möglichen alternativen Realisierungen von logischen elektrischen Bau-elementen gesucht [1]. Das Verhalten von elektrischen Bauelementen auf diesen Längenskalen wird von der Position und räumlichen Verteilung einzelner Atome und Defekte beeinflusst [2]. Die Grenze dieser Entwicklung ist spätestens bei der Verwendung einzelner Atome zur Übertragung von Informationen erreicht. Der Ladungstransport in elektrischen Leitern wird durch grundlegende mikroskopische physikalische Prozesse wie der Elektron-Phonon-, Elektron-Elektron- und der Potential-Streuung an Defekten bestimmt. Um Streumechanismen und deren physikalische Prozesse zu untersuchen, wurden in den letzten Jahrzehnten eine Vielzahl von Transportuntersuchungen unternommen, die darauf basieren, StromSpannungskennlinien aufzunehmen und aus deren Charakteristik in Abhängigkeit von weiteren Variablen wie einem von außen angelegten magnetischen Feld oder der Temperatur mikroskopische Streueigenschaften der betrachteten Proben abzuleiten. Der räumliche Verlauf des Potentials und elektrischen Stromes innerhalb der Proben bleibt bei diesen Verfahren jedoch unbekannt. Diese Lücke kann durch verschiedene Rastersondentechniken geschlossen werden. Diese ermöglichen einen Einblick in lokale elektronische und topographische Eigenschaften von Oberflächen im thermodynamischen Gleichgewicht wie auch im Nicht-Gleichgewicht. So konnte z.B. die Ionisierung einzelner Störstellen in Galliumarsenid und das daraus in der unmittelbaren Umgebung resultierende elektrostatische Potential an der Oberfläche analysiert [3] oder auf Silberfilmen der Prozess der Elektromigration beobachtet werden [4]. Ein zentraler Aspekt dieser Arbeit ist es, das Verfahren der Rastertunnelpotentiometrie (engl. Scanning Tunneling Potentiometry, STP) anzuwenden, welches 1986 von Muralt und Pohl entwickelt worden ist [5]. Dies kombiniert die atomare räumliche Auflösung eines Rastertunnelmikroskops (engl. Scanning Tunneling Microscop, STM) mit einer hohen energetischen Auflösung von bis zu wenigen $\mu \mathrm{eV}$, um den Spannungsabfall und den räumliche Variationen des elektrochemischen Potentials an 
einer stromtragenden Oberfläche zu untersuchen. Das zunehmende Interesse an den physikalischen Prozessen des Ladungstransportes hat zwar in den letzten Jahren zu verschiedenen technischen Realisierungen [6-9] und Messungen an verschiedenen Probensystemen geführt, jedoch gehört die Methode noch nicht zu den standardisierten Messverfahren der Rastertunnelmikroskopie.

Um einen Zugang zu Transporteigenschaften einer möglichst sauberen Oberfläche zu erhalten, ist eine gut definierte Umgebung, wie das Ultra-Hoch-Vakuum (UHV) sinnvoll. Zur Analyse der Streueigenschaften von Defekten ist es außerdem wünschenswert Transportexperimente bei niedrigen Temperaturen durchzuführen, um den Einfluss von ElektronPhonon-Streuprozessen zu minimieren.

In dieser Arbeit wird für die Untersuchung lokaler Transportfelder auf Längenskalen von einigen $100 \mathrm{~nm}$ bis in den atomaren Bereich epitaktisch gewachsenes ein- und zweilagiges Graphen auf der SiC(0001)-Oberfläche verwendet. Abbildung 1 zeigt einen $250 \times 250 \mathrm{~nm}^{2}$ große Topographie der Oberfläche sowie einen atomar aufgelösten Bereich innerhalb einer zweilagigen Graphen-Insel.

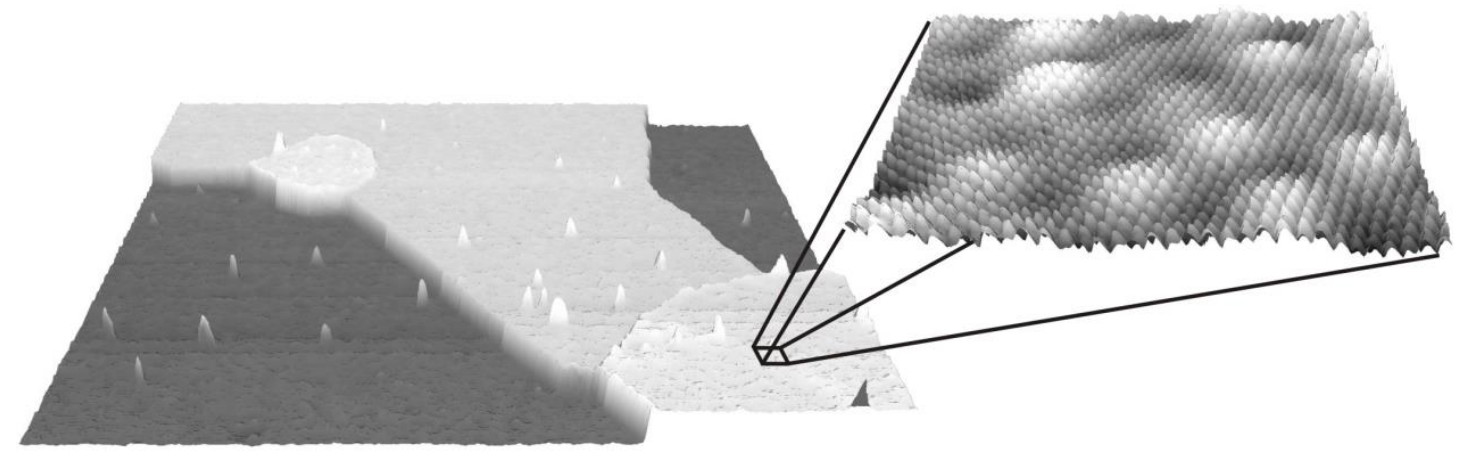

Abbildung 1: Dreidimensionale Darstellung eines 250×250 $\mathrm{nm}^{2}$ Ausschnittes epitaktisch gewachsener Graphen-Lagen auf $\mathrm{SiC}(0001)$ und $8 \times 8 \mathrm{~nm}^{2}$ großer Bereich der Oberfläche von zweilagigem Graphen.

Dieses Probensystem dient als prototypisches Modellsystem für die erstmalige Untersuchung von stromtragenden zweidimensionalen Leitern unter UHV-Bedingungen bei tiefen Temperaturen mit Hilfe der Rastertunnelpotentiometrie (6 K). Außerdem besteht aufgrund seiner möglichen zukünftigen technologischen Relevanz die grundlegende Frage, welchen Einfluss Defekte wie Substratstufen oder mögliche topographische und elektronische Inhomogenitäten auf den Ladungstransport haben. 
Kapitel 1 gibt eine kurze theoretische Einführung in die elektronischen Eigenschaften von Graphen.

Kapitel 2 verschafft einen Überblick über die theoretischen und experimentellen Grundlagen der in dieser Arbeit verwendeten Messmethoden. Insbesondere wird die Messgröße der Rastertunnelpotentiometrie in Verbindung mit verschiedenen Transportregimen diskutiert.

Kapitel 3 widmet sich den spezifischen topographischen und elektronischen Eigenschaften von epitaktisch gewachsenem Graphen auf der SiC(0001)-Oberfläche. Dabei wird zunächst in Kapitel 3.1 bis 3.3 auf die Eigenschaften des Substrates und bisherige Erkenntnisse im Bezug auf das Wachstum und der elektronischen Eigenschaften eingegangen. Die Umsetzung der Probenpräparation, die Kontaktierung und Charakterisierung wird in Kapitel 3.4 beschrieben. In Kapitel 3.5 werden die durch die Rastertunnelmikroskopie gewonnenen Erkenntnisse der topographischen und elektro-nischen Eigenschaften der verwendeten Proben dargelegt.

Kapitel 4 beschäftigt sich mit einem bisher in Kombination mit der Rastertunnel-potentiometrie nur wenig beachteten Effekt, der Thermospannung im Tunnelkontakt, welche zur Analyse der Streuung von Elektronen bei der Fermi-Energie genutzt werden kann. Dies wird auf einer bisher gut untersuchten metallischen Oberfläche, einem $\mathrm{Cu}(111)-K r i s t a l l$, demonstriert und Ergebnisse zur Thermospannung auf epitaktischen Graphen vorgestellt.

Kapitel 5 behandelt die lokalen Transporteigenschaften von epitaktischem Graphen. Es werden zum einen der Kontaktwiderstand zwischen den Kontakten und dem Graphen durch Messungen mit Hilfe der Raster-Kelvin-Mikroskopie ermittelt (Kapitel 5.1) und zum anderen die räumliche Verteilung des elektrochemischen Potentials aus den gewonnenen STP-Daten vorgestellt und der Einfluss der Thermospannung diskutiert (Kapitel 5.2 \& 5.3). Kapitel 5.4 ermittelt im Rahmen des ohmschen Gesetzes quantitativ die spezifischen Leitfähigkeiten von ein- und zweilagigen Graphenflächen und verschieden auftretenden Defekten. Die lokalen Eigenschaften des elektrochemischen Potentials an Substratstufen auf einlagigem Graphen sowie Übergängen zwischen ein- und zweilagigem Graphen im Bereich der Fermi-Wellenlänge werden in Kapitel 5.5 besprochen.

Kapitel 6 fasst die Ergebnisse dieser Arbeit zusammen. 


\section{Inhaltsverzeichnis}

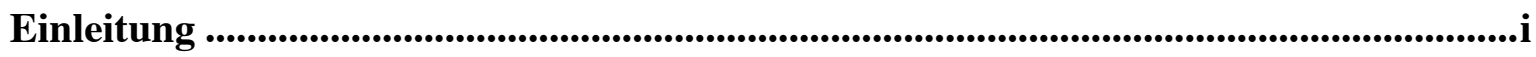

Inhaltsverzeichnis ..............................................................................................................................iv

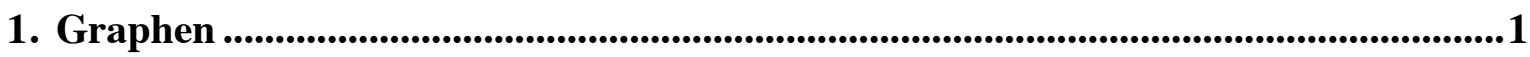

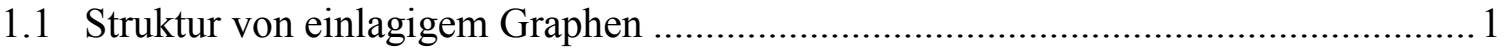

1.2 Elektronische Eigenschaften - Tight-Binding-Modell............................................. 2

1.3 Lineare Näherung am K-Punkt........................................................................ 4

1.4 Struktur und elektronische Eigenschaften von zweilagigem Graphen ....................... 6

2. Experimentelle Methoden und deren theoretische Grundlagen ...............................11

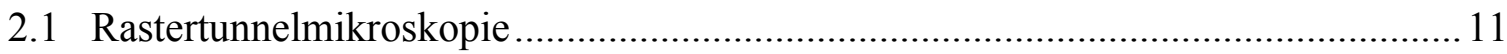

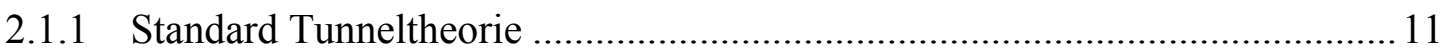

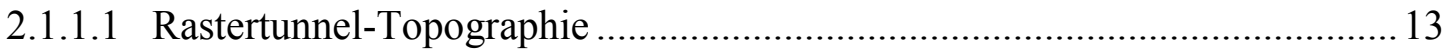

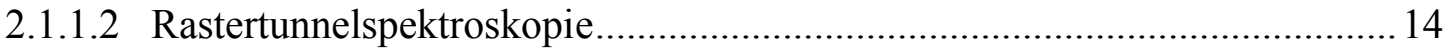

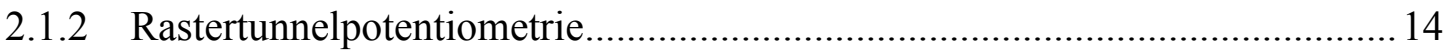

2.1.2.1 Relevante Längenskalen und Transportregime ........................................... 16

2.1.2.2 Konzept des lokalen elektrochemischen Potentials zur Beschreibung des

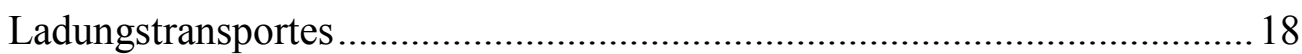

2.1.2.3 Beschreibung des elektrischen Transportes nach der Drude-Sommerfeld-

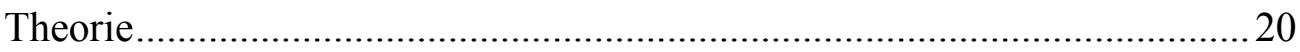

2.1.2.4 Ballistischer Transport und die Streuung an einzelnen Störstellen und

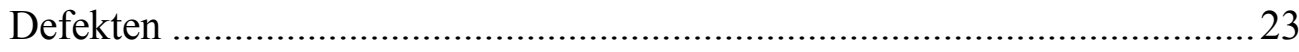

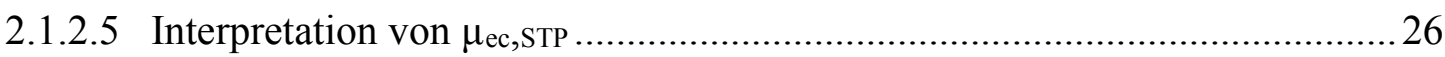

2.1.3 STM-Apparatur und technische Realisierung der Rastertunnelpotentiometrie

\section{Epitaktisches Graphen auf SiC: Wachstum, strukturelle und elektronische}

Eigenschaften

3.1 Eigenschaften des SiC-Substrates

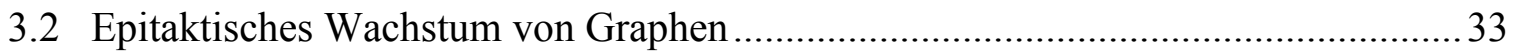

3.3 Strukturelle und elektronische Eigenschaften der $(6 \sqrt{3} \times 6 \sqrt{3}) \mathrm{R} 30^{\circ}$-Rekonstruktion sowie von ein- und zweilagigem epitaktischem Graphen ..................................... 38

3.4 Probenpräparation und Charakterisierung ........................................................... 43

3.5 Topographische und elektronische Untersuchung von ein- und zweilagigem Graphen mit dem Rastertunnelmikroskop ...........................................................5

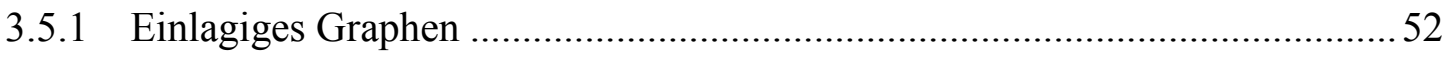

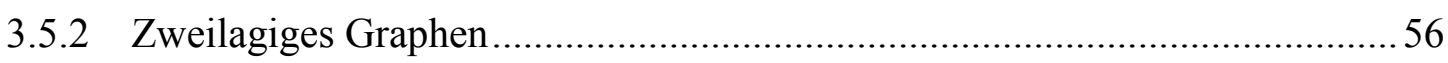


3.5.3 Weitere Defekte - Eigenschaften von ein- und zweilagigem Graphen sowie der Zwischenschicht 60

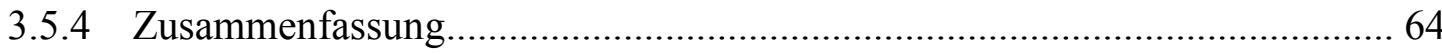

4. Thermospannung auf $\mathrm{Cu}(111)$ sowie epitaktischem Graphen ................................. 65

4.1 Einfluß der Thermospannung auf Tieftemperatur-Potentiometrie-Messungen ........ 65

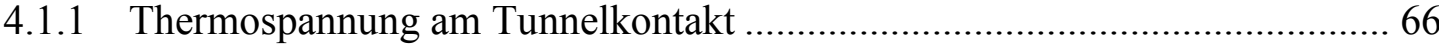

4.1.2 Charakterisierung der Thermospannung auf $\mathrm{Cu}(111)$................................... 67

4.2 Thermospannungssignaturen auf Graphen ............................................................ 71

5. Ladungstransportmessungen von der atomaren bis zur mesoskopischen Skala auf epitaktischem Graphen .................................................................................8 80

5.1 Untersuchung des Graphen-Metall-Kontaktwiderstandes auf semi-isolierendem

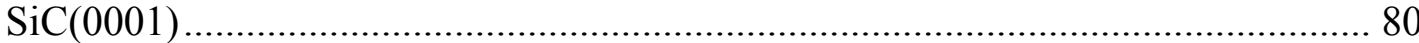

5.2 Rastertunnelpotentiometrie auf epitaktischen Graphen mit lateraler Querspannung 89

5.2.1 Thermospannungsanteil an Ladungstransportmessungen mit der Rastertunnelpotentiometrie sowie Grenzen der Auflösung .................................... 96

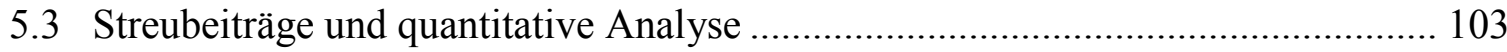

5.3.1 Selbstkonsistente Modellierung im Rahmen des ohmschen Gesetzes.......... 105

5.3.2 Diskussion und Einordnung in bisherige Ladungstransportuntersuchungen 112 5.4 Verlauf des elektrochemischen Potentials in der Umgebung von lokalisierten

Defekten 117

6. Zusammenfassung und Ausblick .......................................................................... 129

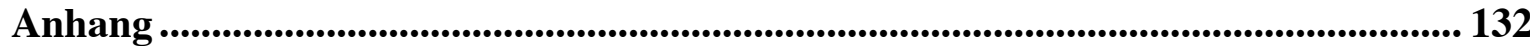

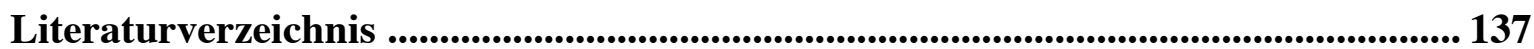

Abkürzungsverzeichnis ............................................................................................................................. 148

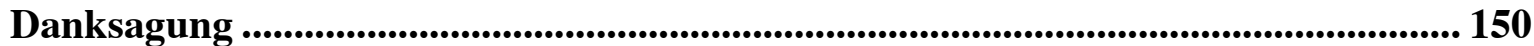

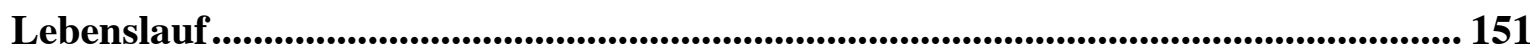





\section{Graphen}

\subsection{Struktur von einlagigem Graphen}

Eine Graphenschicht besteht aus einer atomaren Lage kovalent gebundener Kohlenstoffatome, welche in einem zweidimensionalen Wabengitter angeordnet sind. Ein Kohlenstoffatom hat vier äußere Valenzelektronen, von denen im Graphengitter drei über die in der Ebene liegenden $\mathrm{sp}^{2}$-Hybridorbitale zur Bindung zu den nächsten Nachbarn beitragen. Das verbleibende Elektron bildet ein einfach besetztes $\mathrm{p}_{\mathrm{z}}$-Orbital, welches senkrecht aus der Bindungsebene zeigt und mit $\mathrm{p}_{z}$-Orbitalen benachbarter Kohlenstoffatome ein delokalisiertes $\pi$-Elektronensystem bildet.

a)

$$
\mathrm{a}_{\mathrm{cc}}=0,142 \mathrm{~nm}
$$

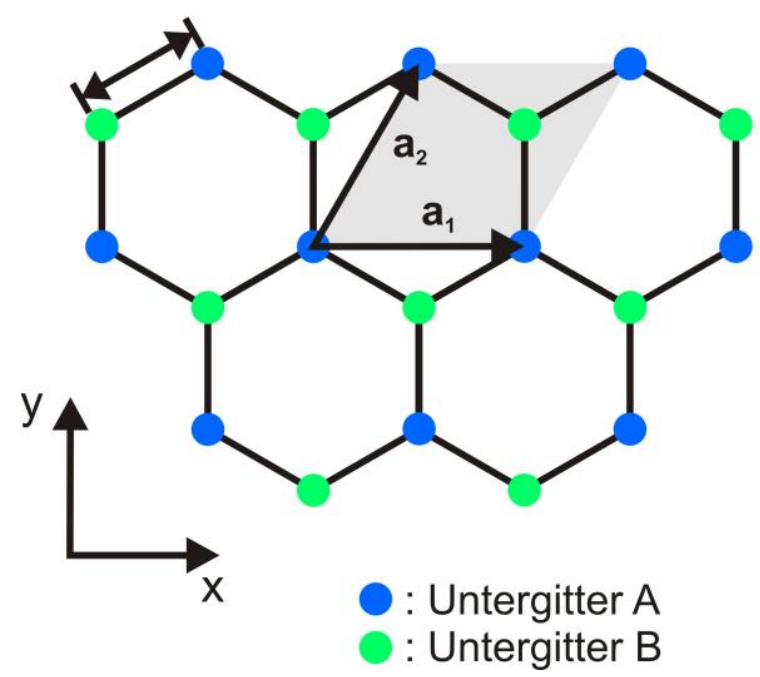

b)

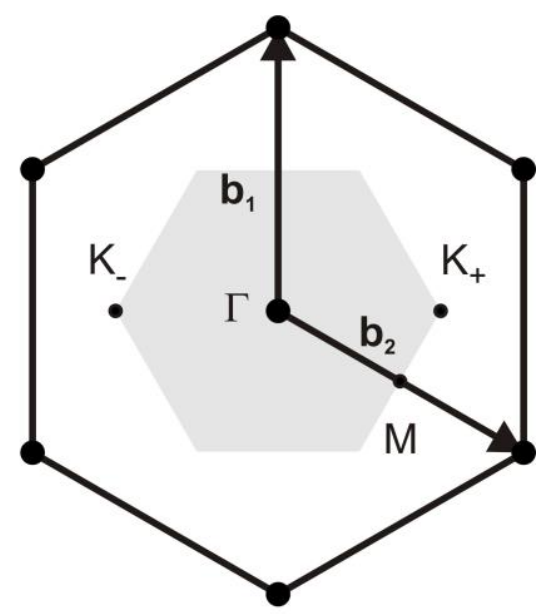

Abbildung 2: (a) Anordnung der Kohlenstoffatome in der Honigwabenstruktur. Die Einheitszelle des Graphengitters besteht aus einem triangulärem Gitter mit zweiatomiger Basis, das von den Gittervektoren und $\mathbf{a}_{1}$ und $\mathbf{a}_{2}$ aufgespannt wird. Die Gitterpunkte der beiden Untergitter sind grün und blau dargestellt. Schwarze Verbindungslinien deuten die $\sigma$-Bindungen zwischen den Kohlenstoffatomen an. Der Abstand zu den nächsten Nachbarn beträgt $\mathrm{a}_{\mathrm{cc}}=0,142 \mathrm{~nm}$. (b) Reziprokes Gitter mit den reziproken Gittervektoren $\mathbf{b}_{\mathbf{1}}$ und $\mathbf{b}_{\mathbf{2}}$. Die erste Brillouin-Zone ist ebenso hexagonal (grau schraffiert) mit den Hochsymmetrie Punkten $\Gamma$, K, M. Jeweils zwei gegenüberliegende Eckpunkte der Brillouin-Zone $\mathbf{K}_{+}$und $\mathbf{K}_{-}$sind nicht äquivalent, da sie nicht mit einem reziproken Gittervektor verbunden werden können. 
Abbildung 2 zeigt die Gitterstruktur von Graphen. Die Anordnung der Atome ist die eines triangulären Bravais-Gitters mit zweiatomiger Basis (A und B). Eine primitive Einheitszelle wird durch die beiden Gittervektoren $\mathbf{a}_{1}$ und $\mathbf{a}_{2}$ aufgespannt, wobei für die Gitterkonstante $\mathrm{a}=\left|\mathbf{a}_{\mathbf{1}}\right|=\left|\mathbf{a}_{\mathbf{2}}\right|=0,246 \mathrm{~nm}$ gilt. Der Abstand zweier benachbarter Kohlenstoffatome beträgt $\mathrm{a}_{\mathrm{CC}}=0,142 \mathrm{~nm}$. Die Honigwabenstruktur ist kein Bravais Gitter, da die Position direkt benachbarter Kohlenstoffatome nicht äquivalent sind bzw. nicht durch einen Gittervektor miteinander verbunden werden können. Das reziproke Gitter ist ebenso hexagonal und wird von den beiden Gittervektoren $\mathbf{b}_{\mathbf{1}}$ und $\mathbf{b}_{\mathbf{2}}$ aufgespannt. Die erste BrillouinZone ist grau schraffiert dargestellt. Das Zentrum wird mit $\Gamma$ bezeichnet und die äquivalenten Eckpunkte der Brillouinzone mit $\mathrm{K}_{+}$und $\mathrm{K}_{-}$. Die zu den Eckpunkten der Brillouin-Zone zugehörigen reziproken Gittervektoren lauten $\mathbf{K}_{\xi}=\xi\left(\frac{4 \pi}{3 a}, 0\right)$ mit $\xi= \pm 1$.

\subsection{Elektronische Eigenschaften - Tight-Binding-Modell}

Die Bandstruktur von Graphen kann mit Hilfe des Modells stark gebundener Elektronen oder auch der sog. Tight-Binding-Näherung beschrieben werden und wurde erstmals schon 1947 von Wallace berechnet [10]. Dabei wird in erster Näherung nur die Wechselwirkung der einfach besetzten $\mathrm{p}_{z}$-Orbitale bzw. $\pi$-Bindungen berücksichtigt. Die innerhalb der Ebene liegenden bindenden $\sigma$-Orbitale bzw. antibindenden $\sigma^{*}$-Orbitale sind energetisch weit unterhalb bzw. oberhalb der Fermi-Energie und tragen daher nicht zu physikalisch relevanten Größen wie der elektrischen Leitfähigkeit bei. Diese werden daher vernachlässigt [11].

Da das Graphengitter aus einer zweiatomigen Basis besteht, kann als Ansatz zur Lösung der Schrödingergleichung $H \Psi_{\boldsymbol{k}}(r)=\varepsilon_{\boldsymbol{k}} \Psi_{\boldsymbol{k}}(\boldsymbol{r})$ eine Linearkombination zweier Blochwellenfunktionen $\Psi_{A}$ und $\Psi_{B}$ gewählt werden [10-12], die den Atomen A bzw. B des jeweiligen Untergitters zugeordnet und mit den komplex konjugierten Koeffizienten $c_{\mathbf{k}}^{\mathrm{A} / \mathrm{B}}$ gewichtet werden:

$$
\begin{aligned}
& \Psi(r)=c_{\boldsymbol{k}}^{A} \Psi_{\boldsymbol{k}}^{A}(\boldsymbol{r})+c_{\boldsymbol{k}}^{B} \Psi_{\boldsymbol{k}}^{B}(\boldsymbol{r}) \\
& =\frac{1}{\sqrt{N}} \sum_{j}\left[e^{i \boldsymbol{k} \boldsymbol{R}_{j}^{A}} c_{\boldsymbol{k}}^{A} \phi\left(\boldsymbol{r}-\boldsymbol{R}_{j}^{A}\right)+e^{i \boldsymbol{k} \boldsymbol{R}_{j}^{B}} c_{\boldsymbol{k}}^{B} \phi\left(\boldsymbol{r}-\boldsymbol{R}_{j}^{B}\right)\right]
\end{aligned}
$$


Die Blochfunktionen werden dabei über die Summe der atomaren Wellenfunktionen $\phi(\boldsymbol{r}-$ $\boldsymbol{R}_{j}^{A / B}$ ) gebildet, wobei $\mathbf{R}_{\mathrm{j}}^{\mathrm{A} / \mathrm{B}}$ der j-te Gitterplatz des Untergitters A bzw. B ist und $\mathrm{N}$ die Anzahl der Einheitszellen angibt. Die Eigenwerte $\varepsilon_{\boldsymbol{k}}$ der Schrödingergleichung können mit Hilfe der Säkulargleichung

$$
\operatorname{det}\left[\boldsymbol{H}-\varepsilon_{\boldsymbol{k}} \boldsymbol{S}\right]=0
$$

bestimmt werden [11]. Dabei ist $\mathbf{S}$ die Überlapp-Integral-Matrix und $\mathbf{H}$ die Transfer-matrix, deren Elemente definiert sind als

$$
H_{i, j}=\left\langle\Psi_{\boldsymbol{k}}^{i}|\boldsymbol{H}| \Psi_{\boldsymbol{k}}^{j}\right\rangle, S_{i, j}=\left\langle\Psi_{\boldsymbol{k}}^{i} \mid \Psi_{\boldsymbol{k}}^{j}\right\rangle \text { miti,j } \in\{A, B\}
$$

Durch Einsetzten der Blochfunktionen und Umformung erhält man für die Transfermatrix und die Überlapp-Integral-Matrix jeweils eine $2 \times 2$ Matrix [11]

$$
H=\left(\begin{array}{cc}
\varepsilon & t f(\boldsymbol{k}) \\
t f(\boldsymbol{k})^{*} & \varepsilon
\end{array}\right), S=\left(\begin{array}{cc}
1 & s f(\boldsymbol{k}) \\
s f(\boldsymbol{k})^{*} & 1
\end{array}\right)
$$

mit $f(\boldsymbol{k})=e^{i k_{x} a / \sqrt{3}}+2 e^{-i k_{x} a / 2 \sqrt{3}} \cos \left(\frac{k_{y} a}{2}\right)$. Dabei ist t das Transfer-Integral oder auch Hopping Element und s das Überlapp-Integral. Im letzten Schritt kann die Dispersionsrelation durch Lösen der Säkulargleichung (1.2) durch Substitution von (1.4) berechnet werden. Als Lösung erhält man

$$
E(\boldsymbol{k})=\frac{\varepsilon \pm t|f(\boldsymbol{k})|}{1 \pm s|f(\boldsymbol{k})|} \text { mit }|f(\boldsymbol{k})|=\sqrt{1+4 \cos \frac{\sqrt{3} k_{x} a}{2} \cos \frac{k_{y} a}{2}+4 \cos ^{2} \frac{k_{y} a}{2}}
$$

Die berechnete Bandstruktur ist in Abbildung 3 (a) für die Brillouin-Zone und in Abbildung 3 (b) als Schnitt entlang der Hochsymmetriepunkte K- $\Gamma-\mathrm{M}-\mathrm{K}$ mit $\mathrm{s}=0$ und $\mathrm{t}=3,033 \mathrm{eV}$ dargestellt. Das Leitungs- und Valenzband d.h. bindendes bzw. antibindendes Band berühren sich an den K-Punkten und sind für die hier gezeigte Näherung mit $\mathrm{s}=0$ symmetrisch um die K-Punkte sowie zweifach entartet. Das Valenzband ist durch die zwei $\pi$-Elektronen je Einheitszelle voll besetzt, während das Leitungsband unbesetzt ist. Die Bandlücke geht gegen null als Konsequenz der Äquivalenz der beiden Atompositionen A und B und damit 
identischer Hauptdiagonalelemente $\varepsilon$ innerhalb der Graphen Einheitszelle [11]. In der Nähe der K-Punkte verläuft die Dispersion in erster Näherung linear, so dass die Quasiteilchen keine effektive Masse besitzen und die elektronischen Eigenschaften äquivalent zu Teilchen ohne Ruhemasse über einen Dirac-Hamiltonian ausgedrückt werden können.

a)

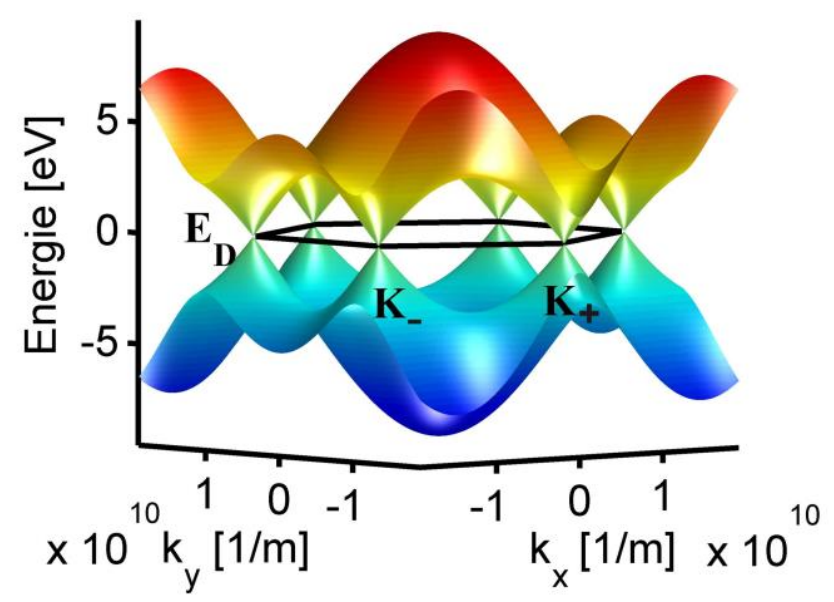

b)

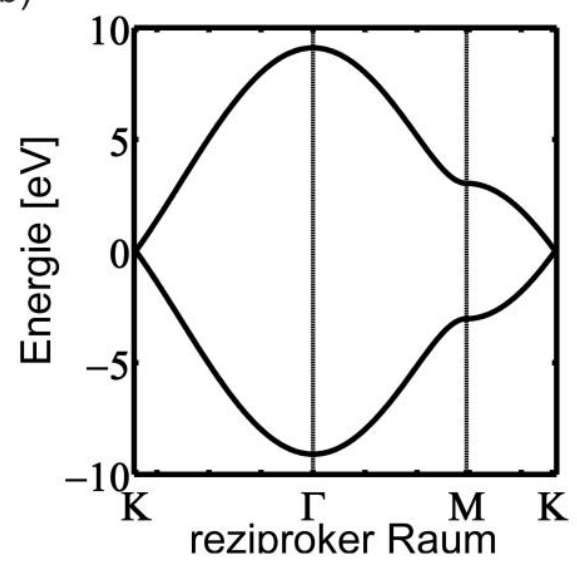

Abbildung 3: Dispersionsrelation $E(\mathbf{k})$ für einlagiges Graphen nach dem Tight-Binding-Modell mit $\varepsilon=$ $0 \mathrm{eV}, \mathrm{t}=3,033 \mathrm{eV}$ und $\mathrm{s}=0$. (a) Leitungs- und Valenzband sind symmetrisch und berühren sich an den inäquivalenten Eckpunkten der hexagonalen Brillouin-Zone $\mathbf{K}_{+}$und $\mathbf{K}_{-}$, welche auch als Dirac-Punkte $E_{D}$ bezeichnet werden. (b) Schnitt der Dispersionsrelation entlang der Hochsymmetriepunkte K-Г-M-K.

\subsection{Lineare Näherung am K-Punkt}

Um die elektronischen Eigenschaften des Graphen besser beschreiben zu können, kann die Dispersionsrelation um die beiden K-Punkte entwickelt werden. Dazu wird der Impuls $\boldsymbol{p}=$ $\hbar\left(\boldsymbol{k}-\boldsymbol{K}_{\xi}\right)$ gemessen vom Mittelpunkt $\mathbf{K}_{\xi}$ eingeführt. Der zugehörige Wellenvektor erhält die Abkürzung q. Die Kopplung t der beiden Untergitter A und B des Graphengitters wird im Hamiltonian (1.4) durch die Nebendiagonalelemente berücksichtigt. Mit Hilfe der Koordinatentransformation und den Koordinaten der nächsten Nachbarn $\boldsymbol{\delta}_{\mathbf{1}}, \boldsymbol{\delta}_{\mathbf{2}}$ und $\boldsymbol{\delta}_{\mathbf{3}}$ lässt sich somit der Ausdruck $f(\boldsymbol{k})$ (vgl. Gleichung (1.4)) linear entwickeln [13]. Berücksichtigt man nur Terme, die linear sind in $\mathbf{p}$, erhält man

$$
f(\boldsymbol{p}) \approx \frac{\sqrt{3} a}{2 \hbar}\left(\xi p_{x}-i p_{y}\right)
$$


und für den Hamilton Operator

$$
\boldsymbol{H}_{\xi}=\frac{\sqrt{3} a t}{2 \hbar}\left(\begin{array}{cc}
0 & \xi p_{x}-i p_{y} \\
\xi p_{x}+i p_{y} & 0
\end{array}\right)
$$

Die zugehörigen Energieeigenwerte und Eigenzustände für das Leitungs- (-) und Valenzband $(+)$ lauten dann

$$
E_{ \pm}= \pm v_{F} p \text { und } \Psi_{ \pm}=\frac{1}{\sqrt{2}}\left(\begin{array}{c}
1 \\
\pm \xi e^{i \xi \varphi}
\end{array}\right) e^{i p r / \hbar}
$$

mit der Definition der Fermigeschwindigkeit $v_{F}=\frac{\sqrt{3} t a}{2 \hbar}$ [13]. Der Impuls wurde dabei in Polarkoordinaten mit $\mathbf{p}=\left(\mathrm{p}_{\mathrm{x}}, \mathrm{p}_{\mathrm{y}}\right)=\mathrm{p}(\cos \varphi, \sin \varphi)$ ausgedrückt. Betrachtet man die Gesamtwellenfunktion $\Psi_{ \pm}$, setzt sich dieser zweikomponentige Vektor aus den beiden Anteilen der Blochwellenfunktionen der jeweiligen Untergitter A, B zusammen. Die relative Amplitude beider Wellenfunktionen zueinander kann neben dem normalen Elektronenspin als zusätzlicher Freiheitsgerad aufgefasst werden, welchen man als Pseudospin bezeichnet $[14,15]$. Dieser steht über den Winkel $\varphi$ in fester Beziehung zum Impuls p der Gesamtwellenfunktion. Um dies zu verdeutlichen, kann der Hamiltonian mit Hilfe der Pauli-Spinmatrizen in die Form

$$
H_{\xi}=\hbar v_{F}\left(\xi \sigma_{x} p_{x}+\sigma_{y} p_{y}\right)=\hbar v_{F} p \boldsymbol{\sigma} \cdot \boldsymbol{n}
$$

gebracht werden, wobei $\boldsymbol{\sigma}=\left(\sigma_{\mathrm{x}}, \sigma_{\mathrm{y}}, \sigma_{\mathrm{z}}\right)$ den Pseudospinvektor und $\mathbf{n}=(\xi \cos \varphi, \sin \varphi, 0)$ den Normalenvektor bildet [13]. Der Hamiltonoperator entspricht damit formal dem Dirac-Hamiltonian für relativistische Teilchen [13].Der Pseudospin hat die Eigenschaft in der Ebene von $\mathbf{p}$ zu liegen und von dessen Richtung abhängig zu sein. Dieser Umstand wird auch als Chiralität der Ladungsträger bezeichnet. Abbildung 4 zeigt schematisch die lineare Dispersionsrelation um die inäquivalenten K-Punkte für n-dotiertes, einlagiges Graphen sowie die Lage des Pseudospins (rote Pfeile) im Leitungsband. 


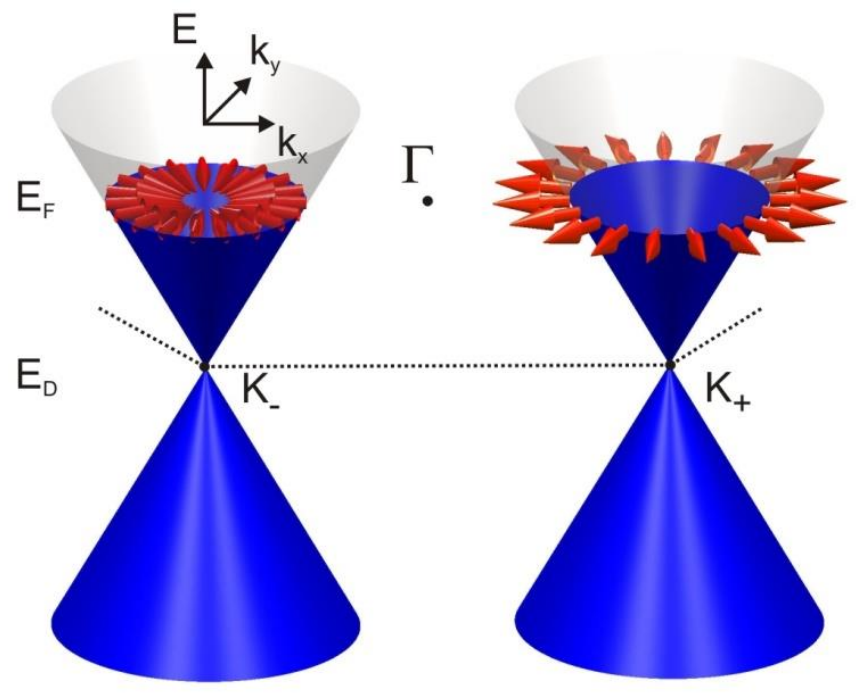

Abbildung 4: Schematische Darstellung der um die K-Punkte in erster Ordnung linear entwickelten Dispersionsrelation für einlagiges Graphen. Rote Pfeile deuten die Richtung des Pseudospins an, welcher für Elektronen im Leitungsband am $\mathbf{K}_{+}$-Punkt in der Ebene parallel zum Wellenvektor $\mathbf{q}$ und am $\mathbf{K}_{-}$-Punkt antiparallel zu q ausgerichtet ist. Elektronenzustände sind daher bei $\mathbf{K}_{+}$rechtshändig bzw. besitzen die Chiralität eins und am K_-Punkt linkshändig mit der Chiralität -1 .

Die Fermi-Energie ist im Gegensatz zum undotierten Fall ins Leitungsband verschoben, sodass die Zustände isotrop im Abstand vom Fermiwellenvektor $\left|\mathbf{q F}_{\mathbf{F}}\right|$ um $\mathrm{K}_{ \pm}$liegen. Im Leitungsband ist der Pseudospin um $\mathrm{K}_{+}$parallel zu p ausgerichtet und um $\mathrm{K}_{-}$antiparallel. Dreht der Impulswellenvektor p um den Winkel $2 \pi$, ändert sich die Phase der Gesamtwellenfunktion um den Betrag $\pi$ [13]. Diese Eigenschaft bezeichnet man auch als Berry-Phase [14], welche maßgeblich die Streueigenschaften von einlagigem Graphen bestimmt und Effekte wie das Klein-Tunneln hervorbringt.

\subsection{Struktur und elektronische Eigenschaften von zweilagigem Graphen}

Zweilagiges Graphen setzt sich aus zwei übereinanderliegenden, zueinander parallelen einlagigen Graphenschichten zusammen. Dabei hängen die elektronischen Eigenschaften des zweilagigen Graphens unter anderem davon ab, welche laterale Position die Atome der beiden Lagen zueinander besitzen [16]. Die bisher experimentell und theoretisch am häufigsten untersuchte Konfiguration ist die sog. AB-Stapelfolge (Bernal-Stacking), bei der zwei der vier Atome $\left(\mathrm{A}_{1}\right.$ und $\left.\mathrm{B}_{1}\right)$ innerhalb der Einheitszelle in der unteren Ebene und zwei Atome $\left(A_{2}\right.$ und $\left.B_{2}\right)$ in der oberen Ebene liegen. Die obere Ebene ist zur unteren um $180^{\circ}$ 
gedreht, so dass die Atome $A_{2}$ und $B_{1}$ direkt übereinanderliegen, während $B_{2}$ und $A_{1}$ über bzw. unterhalb keinen Partner besitzen (vgl. Abbildung 5).

a)

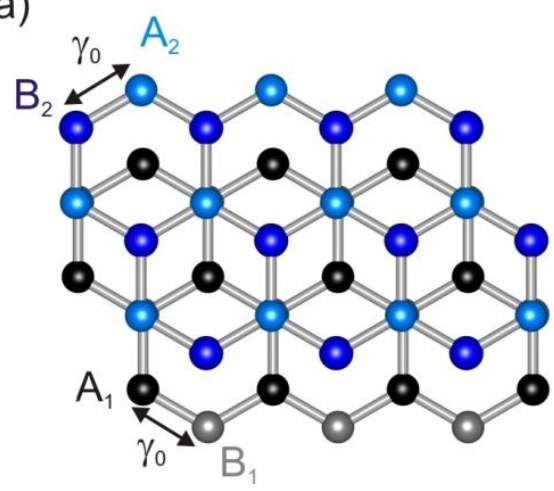

b)

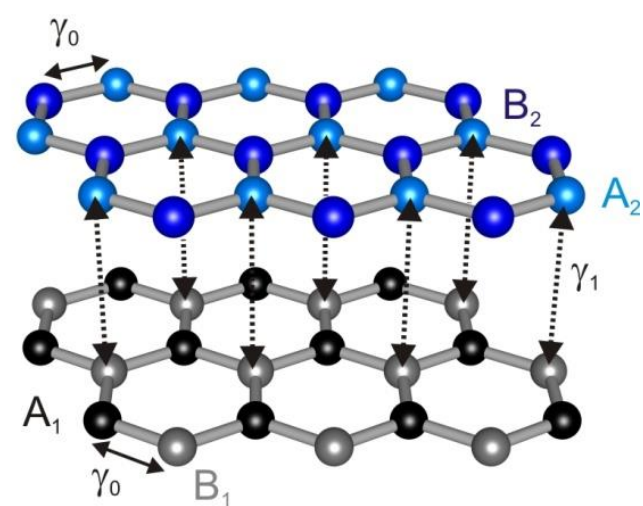

Abbildung 5: Geometrische Anordnung von zweilagigem Graphen in AB-Stapelfolge in der Draufsicht (a) und Seitenansicht (b). Atome der unteren Lage sind mit $\mathrm{A}_{1}$ und $\mathrm{B}_{1}$ und der oberen Lage mit $\mathrm{A}_{2}$ und $\mathrm{B}_{2}$ bezeichnet. Im vereinfachten Tight-Binding-Modell koppeln nächste Nachbarn innerhalb der Ebenen über $\gamma_{0}$, und direkt untereinander liegender Atome $\mathrm{A}_{2}-\mathrm{B}_{2}$ über $\gamma_{1}$.

Die starke Überlagerung der $\mathrm{p}_{\mathrm{z}}$-Orbitale an den Positionen $\mathrm{B}_{1}$ und $\mathrm{A}_{2}$ führt im Vergleich zu einlagigem Graphen zu zwei zusätzlichen Bändern [17]. Bei der Berechnung der Bandstruktur von zwei-lagigem Graphen mit dem Tight-Binding-Ansatz wird analog zu einlagigem Graphen vorgegangen, jedoch können unterschiedliche Hoppingparameter bzw. Kopplungs-konstanten mit berücksichtigt werden. Berücksichtig man die Kopplung unmittelbarer Nachbarn in der Ebene mit dem Kopplungsparameter $\gamma_{A_{1} B_{1}}=\gamma_{A_{2} B_{2}}=\gamma_{0}$ sowie den größten Überlapp zwischen zwei Ebenen mit $\gamma_{A_{2} B_{1}}=\gamma_{1}$, erhält man für den Hamiltonian den Ausdruck

$$
H=\left(\begin{array}{cccc}
-\frac{1}{2} \Delta & 0 & 0 & v_{F} \pi^{*} \\
0 & \frac{1}{2} \Delta & v_{F} \pi & 0 \\
0 & v_{F} \pi^{*} & \frac{1}{2} \Delta & \xi \gamma_{1} \\
v_{F} \pi & 0 & \xi \gamma_{1} & -\frac{1}{2} \Delta
\end{array}\right)
$$

mit $\pi=p_{x}+i p_{y}$ und $\pi^{*}=p_{x}-i p_{y}$, dem Valley-Index $\xi= \pm$ für $\mathrm{K}_{ \pm}$und der Fermigeschwindigkeit $v_{F}=a \gamma_{0}(\sqrt{3} / 2) / \hbar$ [18]. Das Symbol $\Delta$ bezeichnet eine mögliche Energiedifferenz zwischen der oberen und unteren Graphenlage, die zum einen künstlich durch ein 
von außen angelegtes elektrisches Feld oder aber durch unterschiedliche Dotierung der beiden Graphenschichten hervorgerufen werden kann. Als Lösung erhält man vier Bänder mit den Energieeigenwerten $E_{\alpha}^{ \pm}(\mathbf{p})$ mit $\alpha=1,2$, wobei $E_{2}^{ \pm}$die Bänder im Valenz- und Leitungsband bezeichnet, deren Bandkante unter bzw. oberhalb der Energie $\gamma_{1}$ liegen. Die Dispersionsrelation lautet

$$
E_{ \pm}^{\alpha}= \pm \sqrt{\frac{\gamma_{1}^{2}}{2}+\frac{\Delta^{2}}{4}+\hbar^{2} v_{F}^{2} q^{2}+(-1)^{\alpha} \sqrt{\frac{\gamma_{1}}{4}+\hbar^{2} v_{F}^{2} q^{2} \cdot\left(\gamma_{1}^{2}+\Delta^{2}\right)}}
$$

Abbildung 6 zeigt die Dispersionsrelation für zweilagiges Graphen mit verschiedenen Tight-Binding Parametern. In (a) beträgt die Potentialdifferenz zwischen beiden Graphenschichten $\Delta=0 \mathrm{eV}$. Für die Fermigeschwindigkeit wurde der Wert $v_{\mathrm{F}}=1,05 \cdot 10^{6} \mathrm{~m} / \mathrm{s}$ und für die Zwischenschicht-Kopplung der Wert $\gamma_{1}=0,48$ eV gewählt [19]. Im Gegensatz zu einlagigem Graphen zeigen die Bänder des zweilagigen Graphens eine parabolische Dispersionsrelation bei Energien nahe von $E_{\mathrm{F}}$. Für $\Delta \neq 0$ erhält man eine Bandlücke der Größenordnung $E_{\mathrm{g}} \approx \Delta$. Um die Transporteigenschaften an zweilagigem Graphen zu diskutieren wird in der Regel ein weiter vereinfachter effektiver $2 \times 2$-Hamiltonian angegeben [13], welcher die energetisch höher gelegenen Bänder nicht mit berücksichtigt. Definiert man sich wiederum einen Pseudospinvektor $\boldsymbol{\sigma}$ und Normalenvektor $\mathbf{n}_{2}=-(\cos 2 \varphi, \xi \sin 2 \varphi, 0)$, erhält man für diesen

$$
H_{B L, \xi}=-\frac{1}{2 m}\left(\begin{array}{cc}
0 & \left(\xi \mathrm{p}_{\mathrm{x}}-i p_{y}\right)^{2} \\
\left(\xi \mathrm{p}_{\mathrm{x}}+i p_{y}\right)^{2} & 0
\end{array}\right)=\left(\frac{p^{2}}{2 m}\right) \boldsymbol{\sigma} \cdot \boldsymbol{n}_{2}
$$

mit den Energieeigenwerte und Eigenzuständen

$$
E_{ \pm}= \pm \frac{p^{2}}{2 m}, \quad \Psi_{ \pm}=\frac{1}{\sqrt{2}}\left(\begin{array}{c}
1 \\
\mp e^{-i 2 \xi \varphi}
\end{array}\right) e^{-\frac{i p r}{\hbar}}
$$


a)

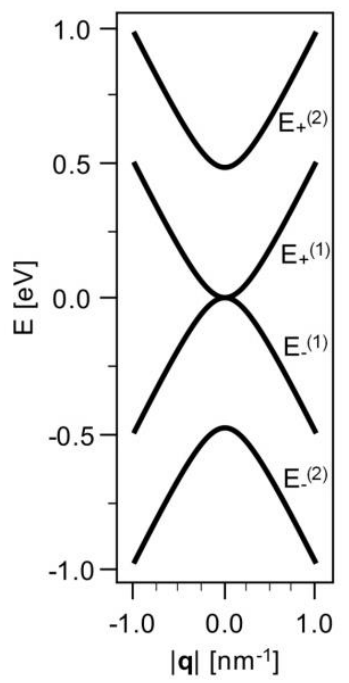

b)

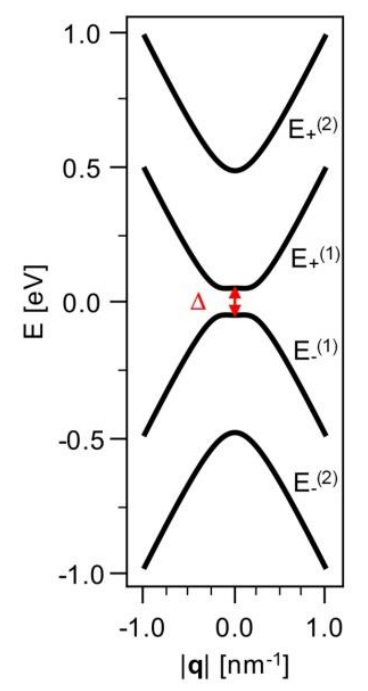

c)

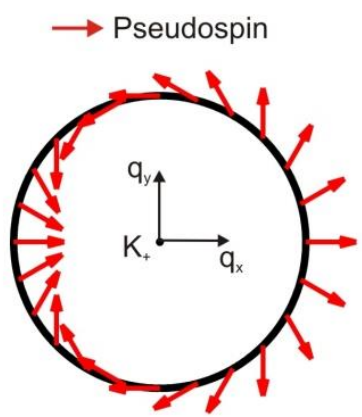

Abbildung 6: $(\mathrm{a}, \mathrm{b})$ Nach vereinfachtem Tight-Binding-Modell berechnete Dispersionsrelation für zweilagiges Graphen mit AB-Stapelfolge. Die Zwischenschichtkopplung beträgt $\gamma_{1}=0,48 \mathrm{eV}$, die Fermigeschwindigkeit $v_{\mathrm{F}}=1,05 \cdot 10^{6} \mathrm{~m} / \mathrm{s}$ und die Potentialdifferenz der beiden Graphenschichten $\Delta=0 \mathrm{eV}$ in (a) und $\Delta=0,1 \mathrm{eV}$ in (b). Die vier Bänder verlaufen für kleine Energien parabolisch. Die Potentialdifferenz kann entweder intrinsisch durch unterschiedliche Dotierung der beiden Schichten zustande kommen, wie es bei epitaktischem Graphen der Fall ist, oder aber durch ein von außen angelegtes, zu den Graphenschichten senkrechtem elektrischen Feld verursacht werden. (c) Schematische Darstellung einer Isoenergiekontur aus (a) um den $\mathrm{K}_{+}$Punkt sowie Pseudospinorientierung (rote Pfeile).

Ein direkter Vergleich zeigt die wesentlichen Unterschiede bzw. Gemeinsamkeiten zu einlagigem Graphen. Im Gegensatz zu einlagigem Graphen besitzt zweilagiges Graphen eine quadratische Dispersionsrelation vergleichbar $\mathrm{zu}$ konventionellen zweidimensionalen Elektronengasen mit einer effektiven Masse. Die Gesamtwellenfunktion lässt sich jedoch ebenso durch einen zweikomponentigen Spinor beschreiben, welcher die Aufenthaltswahrscheinlichkeit der Quasiteilchen auf den Untergittern $\mathrm{A}_{1}$ und $\mathrm{B}_{2}$ beschreibt. Dessen zusätzlicher Freiheitsgrad kann wiederum als Pseudospin ausgedrückt werden, welcher ebenso an p gekoppelt ist, sodass man hier auch von chiralen Ladungsträgern spricht. Der Pseudospin liegt ebenso wie bei einlagigem Graphen in der Ebene, beschreibt aber die relative Amplitude der beiden Wellenfunktionen auf der oberen und unteren Graphen-Lage. Im Gegensatz zu einlagigem Graphen beträgt der Phasenschub der Gesamtwellenfunktion bei Drehung von $\mathbf{p}$ um den Winkel $2 \pi$ ebenso $2 \pi$. 


\section{Experimentelle Methoden und deren theoretische Grundlagen}

\subsection{Rastertunnelmikroskopie}

\subsubsection{Standard Tunneltheorie}

Die theoretische Beschreibung des Tunnelprozesses beruht auf der Arbeit von John Bardeen aus dem Jahr 1961, in der die Tunnelwahrscheinlichkeit zwischen zwei makroskopischen, planparallelen Tunnelkontakten nach der sogenannten Transfer-Hamilton-Näherung beschrieben worden ist [20]. Diese Theorie lässt sich auf andere Geometrien übertragen, wie sie z.B. im STM-Experiment vorkommen.

Nach Bardeen wird der Tunnelstrom als Transfer der Elektronen zwischen ungestörten Proben- und Spitzenzuständen berechnet. Unter Verwendung der zeitabhängigen Schrödingergleichung und Fermis Goldener Regel ist die Rate w der durch die Barriere tunnelnden Elektronen gegeben über

$$
w_{\mu \nu}=\frac{2 \pi}{\hbar}\left|M_{\mu \nu}\right|^{2} \delta\left(E_{\nu}-E_{\mu}\right)
$$

wobei das Transfermatrixelement $\mathrm{M}_{\mu v}$ sich angeben lässt zu

$$
\left|M_{\mu \nu}\right|=\frac{\hbar}{2 m} \int_{S}\left(\chi_{\nu}^{*} \nabla \Psi_{\mu}-\Psi_{\mu} \nabla \chi_{\nu}^{*}\right) \mathrm{d} \mathbf{S} .
$$

Die Integration wird dabei über die Separationsfläche S ausgeführt, welche vollständig im Bereich zwischen Spitze und Probe liegt. Die $\delta\left(E_{v}-E_{\mu}\right)$-Funktion stellt sicher, dass nur Tunnelprozesse zwischen Elektronen gleicher Energie stattfinden, d.h. nur elastische Tunnelprozesse berücksichtigt werden. Bei einer gegebenen Probenspannung $U_{\text {Bias }}$ kann der Tunnelstrom über ein bestimmtes Energieintervall $e U_{\text {Bias }}$ durch die Summation aller Eigenenergien im betreffenden Energieintervall nach folgendem Ausdruck berechnet werden: 


$$
I=\frac{2 \pi}{\hbar} \sum_{\mu, \nu} f_{\mathrm{S}}\left(E_{\nu}^{\mathrm{S}}\right)\left[1-f_{\mathrm{P}}\left(E_{\mu}^{\mathrm{P}}+e U_{\mathrm{Bias}}\right)\right]\left|M_{\mu \nu}\right|^{2} \delta\left(E_{\nu}-E_{\mu}\right) .
$$

Die Besetzungsverteilung der Elektronen der Spitze bzw. Probe bei den jeweiligen Energien und Temperaturen wird dabei im thermischen Gleichgewicht durch die jeweiligen Fermi-Dirac-Verteilungen $f_{\mathrm{S}}(E, T)$ der Spitze bzw. $f_{\mathrm{P}}(E, T)$ der Probe beschrieben.

Nach Gleichung (2.3) finden Tunnelprozesse zwischen Zuständen gleicher Energie nur statt, wenn einer der Spitzen bzw. Probenzustände besetzt und der andere unbesetzt ist. Die Wahrscheinlichkeit eines solchen Überganges ist dabei durch das Betragsquadrat des Tunnelmatrixelementes gegeben. Um eine quantitative Berechnung des Tunnelstroms durchführen und mit experimentellen Daten vergleichen zu können sind weitere Annahmen sowie Vereinfachungen nötig, da zunächst einmal die im Tunnelmatrixelement vorkommenden Wellenfunktionen der Spitze und Probe unbekannt sind. Nach J. Tersoff und D. R. Hamann [21] lässt sich die Gleichung (2.3) für kleine Tunnelspannungen $\left(e U_{\mathrm{Bias}} \ll E_{\mathrm{F}}\right)$ sowie niedrige Temperaturen $\left(k_{\mathrm{B}} T \ll E_{\mathrm{F}}\right)$ weiter vereinfachen zu

$$
I_{\mathrm{T}}=\frac{2 \pi e^{2}}{\hbar} U_{\mathrm{Bias}} \cdot \sum_{\mu, \nu}\left|M_{\mu, \nu}\right|^{2} \delta\left(E_{\mu}^{\mathrm{P}}-E_{\mathrm{F}}\right) \delta\left(E_{\nu}^{\mathrm{S}}-E_{\mathrm{F}}\right) .
$$

Im zweiten Schritt schlugen Tersoff und Hamann vor, die im Allgemeinen unbekannte Wellenfunktion der Spitze durch radialsymmetrische s-förmige Kugelwellen um den Krümmungsmittelpunkt der Spitze $r$ anzunehmen sowie die Oberflächenzustände durch Wellenfunktionen zu beschreiben, die senkrecht zur Oberfläche exponentiell ins Vakuum abklingen:

$$
I_{\mathrm{T}}=\propto e U_{\mathrm{Bias}} \cdot \rho_{\mathrm{S}}\left(E_{\mathrm{F}}\right) \cdot \sum_{\mu}\left|\Psi_{\mu}\left(\boldsymbol{r}_{\mathrm{S}}\right)\right|^{2} \delta\left(E_{\mu}^{P}-E_{\mathrm{F}}\right)
$$

Der resultierende Tunnelstrom ist daher proportional zur angelegten Probenspannung, zur Zustandsdichte der Spitze bei der Fermienergie, welche im relevanten Energiebereich als konstant angenommen wird, sowie proportional zur Summe über alle Probenzustände. Diese wird als lokale Zustandsdichte (engl. Local Density of States, LDOS) bei der betrachteten Energie (hier: $E_{\mathrm{F}}$ ) bezeichnet: 


$$
\operatorname{LDOS}_{P}\left(\boldsymbol{r}_{\mathrm{S},} E_{\mathrm{F}}\right) \equiv \sum_{\mu}\left|\Psi_{\mu}\left(\boldsymbol{r}_{\mathrm{S}}\right)\right|^{2} \delta\left(E_{\mu}^{\mathrm{P}}-E_{\mathrm{F}}\right)
$$

Um auch für größere Probenspannungen bzw. Energiebereiche, wie sie z. B. bei der Untersuchung von Halbleitern aber auch metallischen Oberflächen eine adäquate theoretische Beschreibung des Tunnelstroms zu erhalten, muss die Energieabhängigkeit des Transfermatrixelementes mit berücksichtigt werden. Daher fügten R. J. Hamers et al. [22] einen energie-, spannungs- und tunnelabstandsabhängigen Transmissionskoeffizienten $T\left(E, e U_{\text {Bias }}, d\right)$ ein, der sich mit Hilfe der Wentzel-Kramers-Brillouin Näherung (WKB) $\mathrm{zu}$

$$
T\left(E, e U_{\mathrm{Bias}}, d\right)=\exp \left\{-\frac{2 d}{\hbar} \sqrt{2 m\left(\frac{\phi_{\mathrm{S}}+\phi_{\mathrm{P}}}{2}+\frac{e U_{\mathrm{Bias}}}{2}-E\right)}\right\}
$$

berechnen lässt. Dieser Ausdruck beinhaltet die von der Energie und angelegten Spannung abhängige Form der Tunnelbarriere sowie deren Größe durch die Angabe einer gemittelten Austrittsarbeit $\phi_{\mathrm{S}}$ der Spitze und Probe $\phi_{\mathrm{P}}$. Somit erhält man nach N. D. Lang [23] bei ersetzten der Summe durch das Integral über das Energieintervall $\left[E_{\mathrm{F}}, e U_{\mathrm{Bias}}\right]$ für den Tunnelstrom den Ausdruck

$$
I_{\mathrm{T}} \propto \int \rho_{S}\left(E-e U_{\mathrm{Bias}}\right) \cdot \operatorname{LDOS}\left(\boldsymbol{r}_{S}, d=0, E\right) \cdot T\left(E, e U_{\mathrm{Bias}}, d\right) d E
$$

In dieser Näherung ist der Tunnelstrom proportional zur integralen lokalen Zustandsdichte im betrachteten Energieintervall und zum spitzen- sowie probenabhängigen Transmissionskoeffizienten.

\subsubsection{Rastertunnel-Topographie}

Um eine Abbildung der Probenoberfläche zu erhalten, wird bei der Rastertunnelmikroskopie das grundlegende Verfahren der Konstant-Strom-Topographie verwendet. Bei einer 
festgelegten Spannungsdifferenz ${ }^{1} U_{\text {Bias }}$ zwischen Spitze und Probe sowie einem vorgegebenen Sollwert des Tunnelstroms, wird die Spitze zeilenweise über die Probenoberfläche gerastert. Der Abstand $z$ zwischen Spitze und Probe wird dabei über einen Regelkreis derart eingestellt, das der gemessene Tunnelstrom dem Sollwert entspricht. In der Regel werden an jedem Messpunkt $(x, y)$ die $z$-Werte $z(x, y)$ für $I_{\mathrm{T}}=I_{\text {soll }}$ aufgenommen und z.B. farbkodiert dargestellt. Die erhaltenen Daten werden als Topographie bezeichnet. Unter der Annahme einer im Höhenprofil flachen Probe gibt die Topographie bei kleinen Spannung die Änderung der LDOS der Probenoberfläche wider. Bei höheren Spannungen werden gemäß Flächen konstanter integraler LDOS im entsprechenden Energieintervall $\left[E_{\mathrm{F}}, e U_{\mathrm{Bias}}\right]$ abgebildet. Zeigt die Oberflächenstruktur zusätzlich Höhenvariationen, erhält man als Messsignal eine nicht triviale Überlagerung aus den räumlichen Höhenvariationen und den räumlichen Änderungen der elektronischen Eigenschaften der Probenoberfläche.

\subsubsection{Rastertunnelspektroskopie}

Eine der am häufigsten verwendeten spektroskopischen Messmethoden in der Rastertunnelmikroskopie ist die Bestimmung der differentiellen Leitfähigkeit des Tunnelkontaktes dI/dU. Diese ist in der Regel abhängig vom Ort $(x, y)$, dem über die Topographie definierten Abstand $z$, sowie der über den Tunnelkontakt abfallenden Spannung $U_{\text {Bias }}$. Um die differentielle Leitfähigkeit zu bestimmen, wird an jedem Messpunkt zunächst bei einem bestimmten Setzstrom $I_{\mathrm{T}}$ und Spannung $U_{\mathrm{Bias}}$ die Topographie eingeregelt und darauf folgend bei festgehaltener Spitzenposition eine $I\left(U_{\text {Bias }}\right)$-Kennlinie aufgenommen. Die Ermittlung der differentiellen Leitfähigkeit erfolgt im Anschluss numerisch durch Ableitung der $I\left(U_{\text {Bias }}\right)$-Kennlinie.

\subsubsection{Rastertunnelpotentiometrie}

Die grundlegende Idee der Rastertunnelpotentiometrie ist in Abbildung 7 dargestellt. Sie besteht darin, den Spannungsabfall an stromdurchflossenen Probenoberflächen mit dem la-

\footnotetext{
${ }^{1} U_{\text {Bias }}$ bezeichnet die von außen angelgete Spannungsdifferenz zwischen Spitze und Probe, es wird das relative Probenpotential im Bezug zur Spitze angegeben, für $U_{\text {Bias }}<0$ tunneln Elektronen von der Probe in die Spitze, für $U_{\text {Bias }}>0$ tunneln Elektronen von der Spitze in die Probe.
} 
teralen Auflösungsvermögen eines Rastertunnelmikroskops zu bestimmen. Die erste Realisierung solch eines Experimentes gelang Muralt und Pohl 1986 vier Jahre nach Erfindung der Rastertunnelmikroskopie. Durch Anlegen einer Querspannung $U_{\text {ext }}$ an eine elektrisch leitfähige Probe kann ein Strom durch die Oberfläche der Probe injiziert werden. Um den lokalen Spannungsabfall $U_{\text {Trans }}(x, y)$ zwischen zwei Orten der Probe zu bestimmen, wird das Spitzenpotential $U_{\mathrm{S}}$ an jedem topographischen Messpunkt $z(x, y)$ bei festgehaltener Spitzenposition und damit konstantem Tunnelwiderstand $R_{\mathrm{T}}$ derart adjustiert, dass der resultierende mittlere Tunnelstrom $\left\langle I_{T}\right\rangle$ zwischen Spitze und Probe verschwindet.

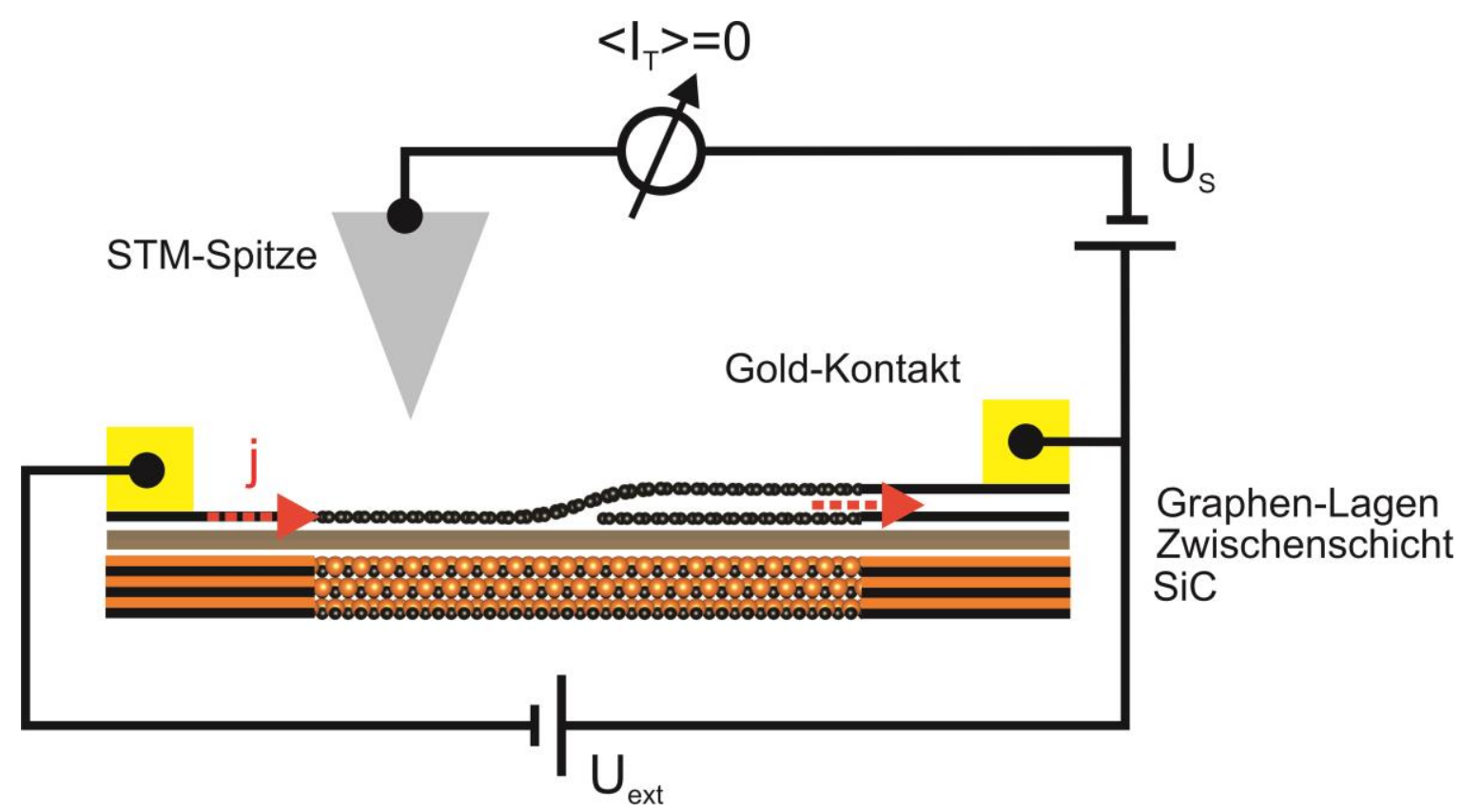

Abbildung 7: Schematische Darstellung des in dieser Arbeit verwendeten Rastertunnelpotentiometrie-Experiments. Die vom Substrat elektrisch isolierten Graphenschichten werden über Goldkontakte elektrisch kontaktiert, so dass sich über eine angelegte Spannung $U_{\text {ext }}$ ein Strom durch die oberen Graphenschichten aufprägen lässt.

Die Idee ist nun, dass dann analog zu einem Potentiometer $U_{S}(x, y)=U_{\text {Trans }}(x, y)$ gilt und damit der lokale Spannungsabfall an einer stromtragenden Probe bestimmt werden kann.

Die Interpretation der durch die Messvorschrift definierten Größe $U_{\mathrm{S}}(x, y)$ bei $I_{\mathrm{T}}=0$ ist im Allgemeinen jedoch nicht trivial. Betrachtet man z.B. den Lösungsansatz für den Tunnelstrom nach Bardeen, so muss nach der Messvorschrift für die Rastertunnelpotentiometrie gelten: 


$$
0=\frac{4 \pi e}{\hbar} \int\left[f_{\mathrm{P}}(E)-f_{\mathrm{S}}\left(E-e U_{\text {Bias }}\right)\right] \rho_{\mathrm{P}}(E) \rho_{\mathrm{S}}\left(E-e U_{\text {Bias }}\right)|M|^{2} d E
$$

Für den in der Potentiometriemessung verwendeten Fall $U_{\text {Bias }}=0$ ist der Tunnelstrom $I_{\mathrm{T}} \neq 0$, wenn die Verteilungsfunktion der besetzten Elektronenzustände von Spitze und Probe bzw. dessen elektrochemischen Potentiale nicht identisch sind. Dies ist genau dann der Fall, wenn Spitze und Probe eine Temperaturdifferenz besitzen oder aber die elektronischen Zustände der Probe durch eine angelegte Querspannung sich im NichtGleichgewicht befinden. Im ersten Fall misst man mit dem Verfahren der Rastertunnelpotentiometrie die sog. Thermospannung $U_{\text {Th }}$ des Tunnelkontaktes. Auf diesen Effekt wird ausführlich in Kapitel 4 eingegangen. Im zweiten Fall definiert die Messvorschrift das lokale elektrochemische Potential $\mu_{\mathrm{ec}}(x, y)$ der Probe im Sinne einer Transport- bzw. NichtGleichgewichtsgröße. Der Begriff des lokalen elektrochemischen Potentials wird in den folgenden Kapiteln im Zusammenhang mit grundlegenden Konzepten des Ladungstransportes erläutert und der erwartete Verlauf der Messgröße auf verschiedenen für den Ladungstransport relevanten Längenskalen und Transportregimen diskutiert.

\subsubsection{Relevante Längenskalen und Transportregime}

Um Transportregime in verschiedene Klassen einzuteilen und damit eine Auswahl der entsprechenden physikalischen Modelle zu treffen, werden Abmessungen des betrachteten Probensystems, wie dessen Länge $l$ und Breite $b$, ins Verhältnis zu charakteristischen Längenskalen gesetzt [24]:

- $\lambda_{\mathrm{F}}$ bezeichnet die Wellenlänge eines Elektrons bei der Fermienergie. Diese liegt in der Größenordnung von einigen Angström bei Metallen und bis zu einigen $10 \mathrm{~nm}$ bei Halbleitern. Bei Graphen ist $\lambda_{\mathrm{F}}$ abhängig von der Elektronenkonzentration $n$ gemäß $\lambda_{\mathrm{F}}=\sqrt{4 \pi / n}$. Epitaktisches Graphen auf $\operatorname{SiC}(0001)$ besitzt eine Fermiwellenlänge von $\lambda_{\mathrm{F}} \approx 10 \mathrm{~nm}$ (Kapitel 4.2).

- $l_{\varphi}$ bezeichnet die Phasenkohärenzlänge der Elektronen, d.h. die Strecke bei welcher die Phase der Elektronenwellenfunktion definiert ist. Inelastische Streuprozesse wie die Elektron-Elektron-Streuung oder Elektron-Phonon-Streuung sorgen für einen Verlust der Phaseninformation. Die Phasenkohärenzlänge für epitaktisches Graphen auf SiC(0001) liegt im Bereich von $l_{\varphi} \approx 140 \mathrm{~nm}$ [25]. 
- $l_{e}$ ist die elastische mittlere freie Weglänge und definiert den mittleren Weg eines Elektrons zwischen zwei elastischen Streuprozessen. Die Phase der Elektronenwellenfunktion bleibt dabei erhalten.

- $\quad l_{\xi}$ gibt die mittlere Länge an, auf welcher die Wellenfunktion lokalisiert ist. Der Grad der Lokalisierung hängt dabei von der Stärke und Dichte der in der Probe vorhandenen Streupotentiale ab.

Grundsätzlich lässt sich durch die charakteristischen Längenskalen der elektronische Transport zwischen mesoskopischen und makroskopischen Transportregimen einteilen.

Bei makroskopischen Transportregimen sind die Probenabmessungen größer als alle anderen für den Transport relevanten Längenskalen $l, b \gg l_{e}, l_{\varphi}, \lambda_{F}, l_{\xi}$.

Das dabei wohl bekannteste makroskopische Transportregime ist das des klassischen diffusiven Transportes, für das $l, b \gg l_{e} \geq l_{\varphi} \gg \lambda_{\mathrm{F}}$ gilt. Die Beschreibung erfolgt in der Regel durch das sog. Drude-Modell. Weitere Transportregime sind die der schwachen und starken Lokalisierung $\left(l, b, l_{\xi} \gg l_{\varphi} \gg l_{e}>\lambda_{\mathrm{F}}\right.$ bzw. $\left.l_{\xi} \approx l_{e} \approx \lambda_{\mathrm{F}}\right)$, bei denen das klassische Drude-Modell versagt und abhängig vom Verhältnis von $l_{\varphi}, l_{\xi}, l_{e}$ und $\lambda_{\mathrm{F}}$ Interferenzeffekte der Elektronen berücksichtigt werden müssen.

Das mesoskopische Transportregime zeichnet sich dadurch aus, dass eine oder mehrere der relevanten Längenskalen $l_{\xi}, l_{e}, l_{\varphi}, \lambda_{\mathrm{F}}$ kleiner als die Länge oder Breite des betrachteten Probensystems ist. Hier kann zwischen diffusivem, ballistischem und quasiballistischem Transport unterschieden werden, wie in Abbildung 7 skizziert ist. Beim mesoskopisch diffusiven Transport sind die Probenabmessungen wie bei makroskopischen Proben $l, b>l_{e}$. Beim ballistischen Transport hingegen gilt $l \ll l_{e}$, d.h. Elektronen werden nur an den Rändern gestreut, innerhalb der Probe findet keine Streuung statt. Eine Beschreibung auf diesen Längenskalen ist mit dem Landauer-Büttiker-Formalismus möglich. Mesoskopische Systeme die weder diffusiv noch ballistisch sind, werden quasiballistisch genannt. 
a)

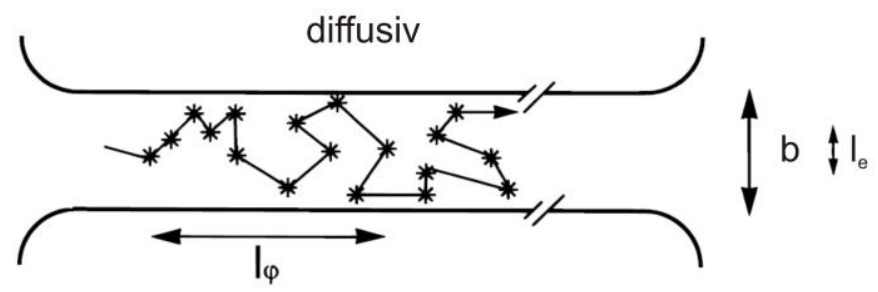

b)
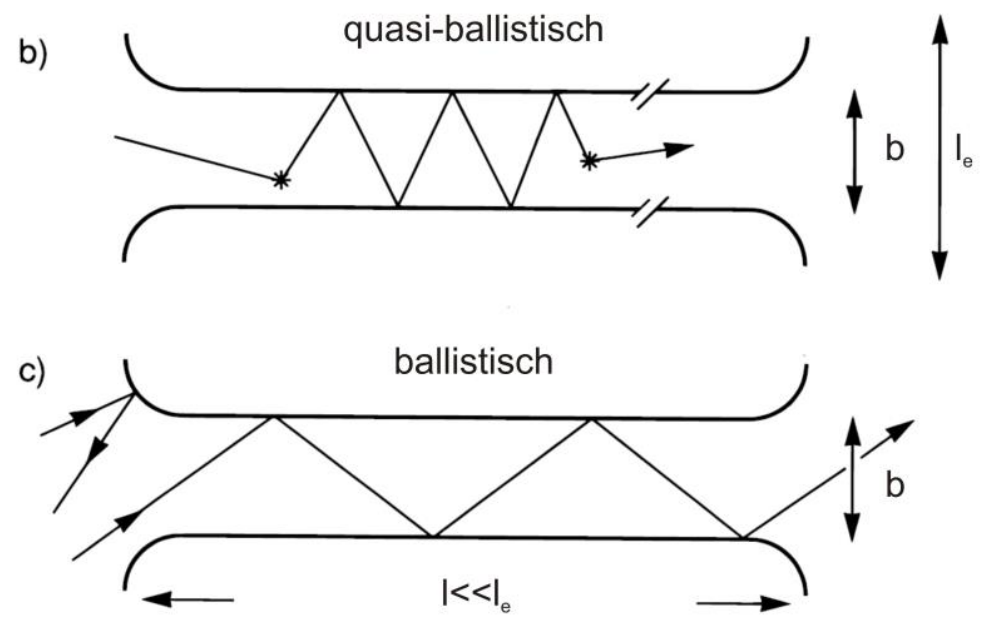

Abbildung 8: Schematische Darstellung der Streuung eines Elektrons im a) diffusiven $\left(l, b>l_{e}\right)$, (b) quasiballistischen und (c) ballistisch $\left(l \ll l_{e}\right)$ mesoskopischen Transportregime in Anlehnung an [26].

\subsubsection{Konzept des lokalen elektrochemischen Potentials zur Beschreibung des Ladungstransportes}

Ladungstransport ist im allgemeinen ein statistischer, ortsabhängiger Nichtgleichgewichtsprozess, für dessen Beschreibung, abhängig von betrachteten Längenskalen in der Regel Konzepte und Größen aus der statistischen Physik als auch aus der Quantenmechanik kombiniert werden. Die elementaren Größen zur Beschreibung sind dabei das elektrostatische Potential $\phi(\boldsymbol{r})$ sowie die Ladungsdichte $n(\boldsymbol{r})$, welche unter der Bedingung der Ladungsneutralität innerhalb eines Leiters die Poisson-Gleichung erfüllen müssen. Diese Größen sind zwar an jedem Punkt eines stromdurchflossenen Systems wohl definiert, jedoch theoretisch wie auch experimentell nicht leicht zugänglich. Dabei beruht der Stromfluss auf einer Nicht-Gleichgewichtsverteilung der Besetzungsfunktion der Elektronen.

Im thermodynamischen Gleichgewicht eines homogenen Festkörpers wird die Besetzungsfunktion $f(\boldsymbol{k})$ der Elektronen in Abhängigkeit des entsprechenden Wellenvektors $\mathbf{k}$ oder der Energie des entsprechenden Zustandes $f(E)$ über die Fermi-Dirac-Verteilung angegeben, für die 


$$
f(E)=\frac{1}{1+e^{\left(\frac{E-\mu_{e c}}{k_{B} T}\right)}} \text { mit } \mu_{e c}=\mu_{c}-e \phi
$$

gilt. ${ }^{2}$ Dabei bezeichnet $\mu_{c}$ das chemische, $\phi$ das elektrostatische Potential und $-e$ die Elementarladung der Elektronen. In einem zum einen inhomogenen und zum anderen sich im Nicht-Gleichgewicht befindlichen System sind jedoch streng genommen weder das chemische noch das elektrochemische Potential definiert. Ebenso wenig kann eine gleichzeitig vom Orts- und Wellenvektor abhängige Verteilungsfunktion $f(\boldsymbol{k}, \boldsymbol{r})$ angegeben werden. Um dennoch den Ladungstransport auf mesoskopischen Längenskalen räumlich aufgelöst beschreiben zu können, überführt man die thermodynamischen Gleichgewichtsvariablen, die die Energie der entsprechenden Zustände festlegen, in lokale Größen $\mu_{e c}(\boldsymbol{r})$ und $\mu_{c}(\boldsymbol{r})$. Dafür teilt man das System in endliche kleine Gebiete ein und nimmt weiterhin an, dass sich die Besetzung der Elektronen durch lokale Quasi-Fermi-Energien und Verteilungen beschreiben lassen. Bei den gewählten Bereichen geht man zum einen davon aus, dass sie groß genug sind, um sie durch statistische, thermodynamische Zustandsvariablen beschreiben zu können und gleichzeitig klein genug, um die Bedingung lokaler Gleichgewichte zu erfüllen [27]. Geht man außerdem von stationären zeitunabhängigen Zuständen aus, lässt sich ein lokales elektrochemisches Potential definieren, welches gegeben ist über

$$
\mu_{\mathrm{ec}}(\boldsymbol{r}):=\mu_{\mathrm{c}}(\boldsymbol{r})-e \phi(\boldsymbol{r})[27]
$$

Das Konzept des lokalen elektrochemischen Potentials und einer damit verbundenen ortsund energieabhängigen Verteilungsfunktion wird bei der theoretischen Berechnung mesoskopischer Transportprobleme verwendet. Auf Längenskalen unterhalb der Fermiwellenlänge bricht dieses Konzept jedoch zusammen, wie nummerische Berechnungen zeigen, und es wird eine Mittelung über Raumbereiche in der Größenordnung der Fermiwellenlänge notwendig [28]. Bis zu welchen Längenskalen der theoretische Ansatz eines lokalen

\footnotetext{
${ }^{2}$ Für gewöhnlich wird bei der Definition der Fermi-Verteilung das chemische Potential verwendet [145]. Dies gilt strenggenommen jedoch nur für homogene Festkörper. Möchte man jedoch im thermodynamischen Sinne die Ladung der Elektronen mit berücksichtigen, ist es angebracht das elektrochemische Potential $\mu_{\mathrm{ec}} \mathrm{zu}$ verwenden. Betrachtet man z.B. einen pn-Übergang im thermodynamischen Gleichgewicht, besitzt dieses ein konstantes elektrochemisches Potential. Aufgrund der unterschiedlichen Dotierung kommt es jedoch zu einer Ladungsverschiebung und zu einem räumlich variierendem elektrostatischem Potential $\phi(\boldsymbol{r})$, so dass ebenso die Eigenergien der Bänder um den Energiebeitrag $e \phi(\boldsymbol{r})$ variieren.
} 
elektrochemischen Potentials und die Verwendung einer auf Basis der Fermiverteilung definierten lokalen Verteilungsfunktion seine Gültigkeit hat, kann nur experimentell überprüft werden. Mit dem Rastertunnelmikroskop hat man die Möglichkeit die elektronischen wie auch Transporteigenschaften auf Längenskalen von einigen $\mu \mathrm{m}$ bis hinunter zu einer lateralen Auflösung im Ångström-Bereich zu untersuchen. Eine ausführliche Darstellung der Nutzung des Konzeptes der Quasi-Fermi-Energien und elektrochemischen Potentiale im Zusammenhang mit dem Ladungstransport auf mesoskopischen Längenskalen findet sich im Buch von S. Datta „Electronic Transport in mesoscopic Systems“ [29]. Auf Grundlage der dort beschriebenen Theorien soll im Folgenden die Theorie des diffusiven und ballistischen Ladungstransportes mit vereinzelten Störstellen dargestellt werden.

\subsubsection{Beschreibung des elektrischen Transportes nach der Drude-Sommerfeld-} Theorie

Der elektrische Transport von Elektronen in Metallen wurde im Jahr 1900 von Paul Drude mit einer klassischen Modellvorstellung eines freien Elektronengases beschrieben und 1933 von A. Sommerfeld und H. Bethe korrigiert, indem sie das Paulische Ausschlussprinzip und die daraus resultierende Fermi-Dirac-Statisitk berücksichtigten [30]. Das DrudeSommerfeld-Modell basiert auf den Annahmen, dass die Elektronen und Ionen des Festkörpers nur durch unabhängige Stöße wechselwirken und die Wechselwirkung der Elektronen untereinander vernachlässigbar ist.

Die mittlere Stromdichte $\mathbf{j}$ durch ein Einheitsvolumen lässt sich durch die mittlere Geschwindigkeit $\boldsymbol{v}_{\boldsymbol{m}}=\hbar\langle\boldsymbol{k}\rangle / m_{e}$ multipliziert mit der Anzahl der Ladungsträger n pro Einheitsvolumen und deren Ladung -e ausdrücken und beträgt

$$
\boldsymbol{j}=-e n \boldsymbol{v}_{\boldsymbol{m}}=-\frac{e n \hbar}{m_{e}}\left(\langle\boldsymbol{k}\rangle-\langle\boldsymbol{k}\rangle_{\mathbf{0}}\right)=-\frac{e n \hbar}{m_{e}} \delta \boldsymbol{k}
$$

Dabei bezeichnet $\hbar\langle\mathbf{k}\rangle_{0}$ die mittlere Impulsverteilung des ruhenden Elektronengases im thermodynamischen Gleichgewicht und $\hbar\langle\boldsymbol{k}\rangle$ die des Nichtgleichgewichts [30]. Einen Stromfluss erhält man damit nur bei einer nicht verschwindenden mittleren Impulsverteilung der Elektronen von $\delta \boldsymbol{k}$. Die zeitliche Änderung des mittleren Impulses setzt sich dabei aus zwei Komponenten zusammen, einer von außen wirkenden Kraft in Form eines konstanten elektrischen Feldes $\boldsymbol{F}=-\boldsymbol{e} \boldsymbol{E}$ und der Streuung der Elektronen an Defekten. Dabei 
wird angenommen, dass im Ensemblemittel unmittelbar nach jedem Stoß die ursprüngliche Impulsverteilung $f(\boldsymbol{k})$ wieder hergestellt ist und die durch das elektrische Feld gewonnene Energie über Phononen an die Umgebung abgegeben wird. Nach dem Relaxationsansatz wird die Änderung des mittleren Elektronenimpulses durch Streuereignisse durch eine mittlere Streuzeit $\tau$ ausgedrückt, so dass gilt

$$
\hbar \frac{d\langle\boldsymbol{k}\rangle}{d t}=\left.\hbar \frac{\partial\langle\boldsymbol{k}\rangle}{\partial t}\right|_{\mathrm{Kraft}}+\left.\hbar \frac{\partial\langle\boldsymbol{k}\rangle}{\partial t}\right|_{\text {Streu }}=-e \boldsymbol{E}-\hbar \frac{\delta \boldsymbol{k}}{\tau}
$$

Im Folgenden werden nur stationäre Prozesse betrachtet, d.h. im zeitlichen Mittel ändert sich die Impulsverteilung der Elektronen nicht, so dass $\frac{d\langle\boldsymbol{k}\rangle}{d t}=0$ gilt. Damit erhält man eine Änderung der Impulsverteilung der Ladungsträger im Vergleich zum Gleichgewichtsfall um

$$
\hbar \delta \boldsymbol{k}=-e \boldsymbol{E} \tau
$$

und mit Gleichung (2.12) das ohmsche Gesetz

$$
\boldsymbol{j}=\frac{n e^{2} \tau}{m} \cdot \boldsymbol{E}=n e \mu=\frac{n e^{2} l}{m v_{\mathrm{F}}} \boldsymbol{E}=\sigma \cdot \boldsymbol{E}
$$

Dabei ist $\mu=\frac{e \tau}{m}$ die Mobilität der Ladungsträger und $l_{\mathrm{mfp}}=v_{\mathrm{F}} \tau$ definiert die mittlere freie Weglänge, die ein Elektron innerhalb der Streuzeit zwischen zwei Streuprozessen zurücklegt. Für die Geschwindigkeit der Elektronen verwendet man die Fermigeschwindigkeit $v_{\mathrm{F}}=\hbar k_{\mathrm{F}} / m$, da nach dem Paulischen Ausschlussprinzip Elektronen nur von besetzten $\mathrm{Zu}$ ständen in unbesetzte streuen können, welche wiederum nur bei der Fermienergie innerhalb der thermischen Verbreiterung von $\sim \mathrm{k}_{\mathrm{B}} \mathrm{T}$ liegen. Die Besetzung der Zustände $\boldsymbol{k}$ eines zweidimensionalen Elektronengases, welches durch die Besetzungsfunktion $f(\boldsymbol{k})$ bestimmt wird, entspricht der einer verschobenen runden Fermi-Fläche, wie in Abbildung 9 (a) dargestellt ist. Für die energetische Verteilung der Elektronen gilt bei angelegtem elektrischen Feld $f(\boldsymbol{k})=f(\boldsymbol{k}+\delta \boldsymbol{k})$. Da nur einige Elektronen durch das elektrische Feld von $\boldsymbol{k}^{-}$Zuständen in $\boldsymbol{k}^{+}$Zustände wechseln, wird bei der Beschreibung von mesoskopischen Trans- 
portproblemen die verschobene Besetzungsfunktion der Elektronen bzw. das elektrochemische Potential in zwei Teile $f\left(\boldsymbol{k}^{+}\right)$und $f\left(\boldsymbol{k}^{-}\right)$, mit der Energie $\mu_{\mathrm{ec}}^{+}$bzw. $\mu_{\mathrm{ec}}^{-}$unterteilt. Dabei tragen nur Elektronen mit Wellenvektoren $\mathbf{k}^{+}$im Energieintervall $\mu_{\mathrm{ec}}^{+}>E>\mu_{\mathrm{ec}}^{-}$in Richtung des elektrischen Feldes zum Nettostrom bei, wie in Abbildung 9 (b) dargestellt ist.

Abbildung 9 zeigt im Rahmen dieser Theorie den räumlichen Verlauf des elektrochemischen Potentials, der quasi-elektrochemischen Potentiale $\mu_{\mathrm{ec}}^{+}$und $\mu_{\mathrm{ec}}^{-}$sowie der elektrostatischen Energie innerhalb eines homogenen Leiters gemäß der beschriebenen Drude-Sommerfeld-Theorie. Da im stationären Fall eines strom-durchflossenen homogenen Leiters die Ladungsdichte konstant ist, variiert auch das chemische Potential bzw. die Anzahl der Ladungsträger nicht und es herrscht Ladungsneutralität. Aufgrund der Tatsache, dass es keine lokalisierten Streuzentren gibt, ergibt sich auch keine räumliche Abhängigkeit der Verteilungsfunktion. Die elektrostatische Potentialdifferenz wird innerhalb der Kontakte durch entsprechende Ladungen gemäß der Poisson-Gleichung aufgebaut. Das elektrochemische Potential folgt dem elektrostatischen während das chemische Potential konstant bleibt, wie in Abbildung 9 (c) illustriert ist. 
a)

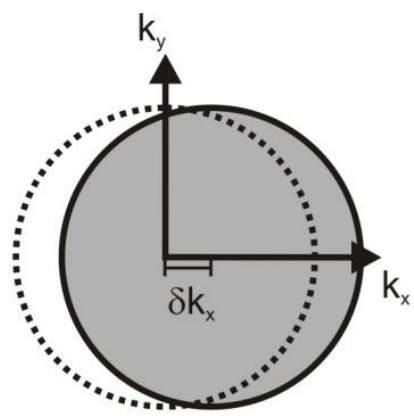

b)

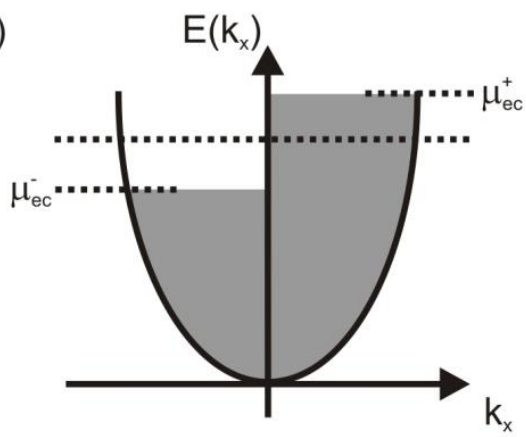

c)

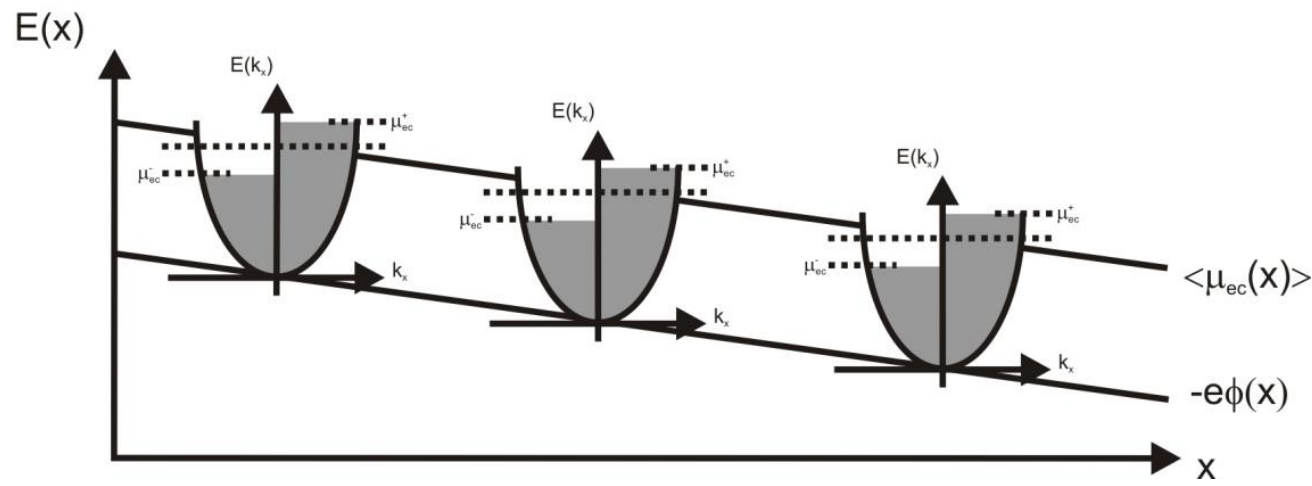

Abbildung 9: (a) Um $\delta k_{\mathrm{x}}$ verschobene Fermi-Verteilung nach dem Drude-Sommerfeld Modell bei einem von außen angelegtem elektrischen Feld $-E_{\mathrm{x}}$. (b) Einteilung der Besetzungsfunktion bzw. des elektrochemischen Potential in zwei separate sogenannte quasi-Potentiale $\mu_{\mathrm{ec}}^{+}(x)$ und $\mu_{\mathrm{ec}}^{-}(x)$. Stromtragende Zustände, die in positiver $\mathrm{x}$-Richtung laufen, sind bis zur Energie $\mu_{e c}^{+}(x)$ besetzt und in negativer $\mathrm{x}$-Richtung bis zur Energie $\mu_{\mathrm{ec}}^{-}(x)$. Nur Elektronen im Energieintervall $\mu_{\mathrm{ec}}^{-}<E<\mu_{\mathrm{ec}}^{+}$tragen zum Nettostrom bei. (c) Verlauf des lokalen elektrochemischen Potentials $\mu_{\mathrm{ec}}(x)$ sowie der elektrostatischen Energie - $e \phi(x)$ an einem makroskopischen, homogenen Leiter. Aufgrund der Bedingung der Ladungsneutralität gilt an jedem $\operatorname{Ort} \mu_{\mathrm{ec}}(x)+$ $e \phi(x)=\mu(x)=$ const. und der Verlauf von $\mu_{\mathrm{ec}}$ entspricht dem Verlauf von $-e \phi$.

\subsubsection{Ballistischer Transport und die Streuung an einzelnen Störstellen und Defekten}

Ein erfolgreiches Konzept zur Beschreibung des Ladungstransportes mesoskopischer Transportregime ist der von Rolf Landauer eingeführte Formalismus. Der grundlegende Ansatz dabei ist anzunehmen, dass die Ursache für den elektrischen Transport von Ladungsträgern innerhalb eines Leiters nicht ein über den Leiter abfallendes elektrisches Feld ist, sondern über die Kontakte in den Leiter injizierte Ladungsträger, die mit einer bestimmten Wahrscheinlichkeit diesen durchqueren [31]. Die Streuung an z.B. Defekten und Störstellen führt zu einer selbstkonsistenten Anhäufung der Ladungsträger und damit innerhalb des Leiters zu einem inhomogenen elektrischen Feld bzw. einer inhomogenen Potentialverteilung [31]. 
Der elektrische Leitwert für einen makroskopischen zweidimensionalen homogenen Leiter der Breite b und Länge 1 sowie einer spezifischen Leitfähigkeit wird durch das ohmsche Gesetz

$$
G=\sigma \frac{b}{l}
$$

beschrieben. Für eine immer kleiner werdende Länge des Leiters erwartet man demnach intuitiv einen kontinuierlich ansteigenden Leitwert. Experimentell beobachtet man jedoch für den Leitwert innerhalb eines ballistischen Leiters unterhalb der mittleren freien Weglänge, dass dieser einen maximalen Wert erreicht, für den

$$
G=\frac{2 e^{2} \cdot M}{h}=\frac{M}{12,9} \mathrm{k} \Omega^{-1}
$$

mit der transversalen Modenanzahl $M \in \mathbb{N}_{>0}$ gilt [29]. Aufgrund der endliche Breite b des Leiters spalten die Elektronenenergien auf und es bilden sich Subbänder aus. Die maximale Anzahl der Moden kann dabei unter der Annahme periodischer Randbedingungen und der Fermiwellenlänge zu $M=\operatorname{Int}\left(\frac{2 b}{\lambda_{F}}\right)$ abgeschätzt werden, wobei Int(x) eine ganze Zahl kleiner x ist [29]. Da innerhalb des ballistischen Leiters keine Streuung der Elektronen auftritt und damit auch kein Spannungsabfall, wird der quantisierte Leitwert bzw. Widerstand $G^{-1}$ und der dazugehörige Spannungsabfall dem Übergang zwischen den Kontakten und dem ballistischen Leiter zugeordnet und auch als Kontaktwiderstand bezeichnet.

Der durch die Geometrie bestimmte maximale Leitwert eines Leiters kann nun durch zusätzliche Defekte wie Fehlstellen, Punktdefekte oder Fremdatome herabgesetzt werden. Diese stören lokal das periodische Potential des Kristalls. Es kommt zur Streuung der Elektronen und einer Herabsetzung des Leitwertes. Quantenmechanisch lassen sich solche Defekte durch Potentialbarrieren beschreiben. Nimmt man eine Barriere innerhalb eines ballistischen Leiters an, dessen Transmission $T$ die mittlere Wahrscheinlichkeit bestimmt, mit welcher ein Elektron diese durchqueren kann, erhält man nach Landauer für den an den Kontakten gemessen Leitwert den Ausdruck 


$$
G=\frac{2 e^{2} \cdot M}{h} \cdot T
$$

Ohne Berücksichtigung der Kontaktwiderstände und Messung des Potentials innerhalb des ballistischen Leiters vor und hinter dem Streuzentrum ist $T$ durch $T /(1-T)$ in Gleichung (3) zu ersetzen.

Abbildung 10 veranschaulicht den erwarteten Verlauf des elektrochemischen Potentials $\mu_{e c}(\boldsymbol{r})$, der elektrostatischen Energie - $e \phi(\boldsymbol{r})$ sowie der Ladungsdichte $n(\boldsymbol{r})$ innerhalb eines ballistischen Leiters mit einem lokalisierten Streuzentrum gemäß S. Datta [29]. Während innerhalb eines ballistischen Leiters keine Spannung abfällt und das elektrochemische Potential konstant ist, wird an einer Barriere ein Teil der ankommenden Elektronen mit 1 $T$ reflektiert, während der andere Teil die Barriere mit der Wahrscheinlichkeit $T$ überquert. Die Anzahl der Elektronen unmittelbar vor und hinter dem Streuzentrum ändert sich und es kommt zu einer abrupten Änderung von $\mu_{\mathrm{ec}}$ an der Barriere wie in Abbildung 10 (a) dargestellt ist. Der Spannungsabfall über die Barriere ist dabei durch die Differenz der elektrochemischen Potentiale $\mu_{1}$ und $\mu_{2}$ über $e U=\mu_{1}-\mu_{2}$ gegeben. Vor der Barriere herrscht ein Ladungsüberschuss und hinter der Barriere eine Verarmung von Ladungsträgern. Dieser Ladungsdipol wird als Landauer- Widerstandsdipol bezeichnet, welcher erstmals 1957 als mikroskopische Ursache für den Restwiderstand in Metallen von R. Landauer theoretisch mit Hilfe der semiklassischen Transporttheorie beschrieben worden ist. Damit innerhalb des Leiters die Bedingung der Ladungsneutralität gewährleistet ist und die lokalen elektrochemischen Potentiale links und rechts von der Barriere entsprechend ohne Streuer konstant ist, muss sich gemäß $\mu_{\mathrm{ec}}(r)=\mu_{\mathrm{c}}(\boldsymbol{r})-e \phi(\boldsymbol{r})$ die Ladungsdichte und das elektrostatische Potential selbstkonsistent adjustieren. Aufgrund einer endlichen Abschirmlänge kann das elektrostatische Potential bzw. die energetische Position der Leitungsbandkanten ES, 1 bzw. ES, 2 links und rechts des Streuzentrums jedoch nicht einer abrupten Änderung des elektrochemischen Potentials folgen, und es kommt zu einem Überschuss an Elektronen vor bzw. einer Verarmung an Elektronen hinter dem Streuzentrum [28, 29]. 
(a)

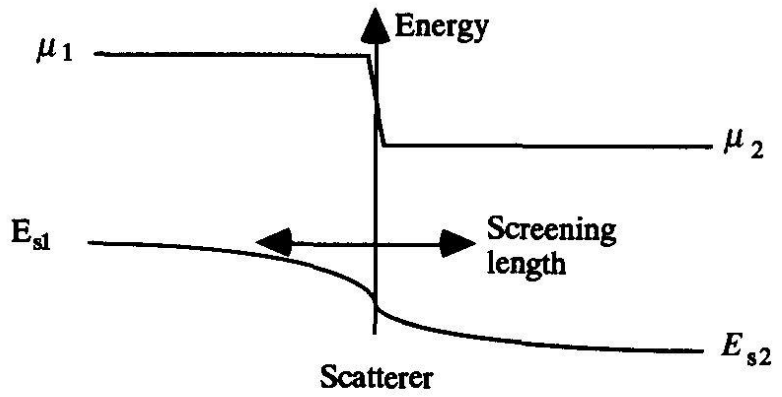

(b)

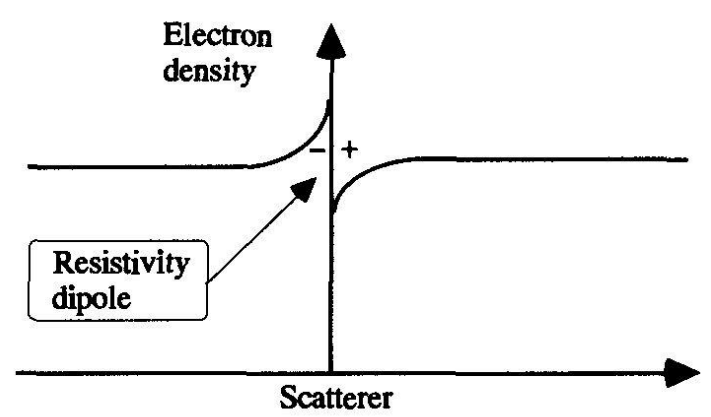

(c)

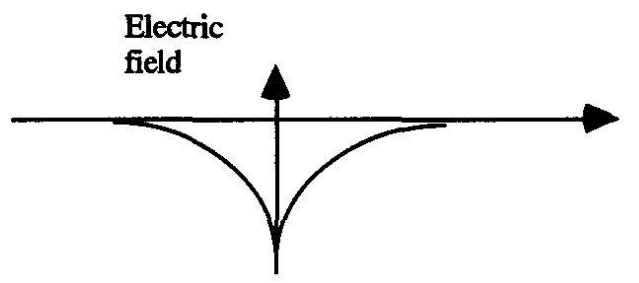

Abbildung 10: (a) Schematischer Darstellung des räumlichen Verlaufes der elektrochemischen Potentiale $\mu_{\mathrm{ec}, 1}$ vor und $\mu_{\mathrm{ec}, 2}$ hinter einer Potentialbarriere, welche in einen ansonsten ballistischen Leiter eingebettet ist, und Darstellung der energetischen Position der Leitungsbandkanten $E_{\mathrm{S}}$ im Fall eines Elektronentransportes von links nach rechts [29]. (b) Ladungsdichte $n(\mathbf{r})$ und (c) dazugehöriges elektrisches Feld [29].

\subsubsection{Interpretation von $\mu_{\mathrm{ec}, \mathrm{STP}}$}

Nach der in Kapitel 2.1.2 vorgestellten Messvorschrift erfolgt ein Abgleich der Besetzungsfunktion der Spitze $f_{\mathrm{S}}(E)$ mit einer vom Ort abhängigen Besetzungsfunktion der Probe $f_{\mathrm{P}}(E, \boldsymbol{r})$. Ist das elektrochemische Potential der Spitze durch eine angelegte Spannung Us derart adjustiert, dass im zeitlichen Mittel kein Tunnelstrom fließt, befindet sich die Spitze im Gleichgewicht. Die in Kapitel 2.1.2.3 eingeführten quasi-elektrochemischen Potentiale der Spitze $\mu_{\mathrm{ec}, \mathrm{S}}^{+}$und $\mu_{\mathrm{ec}, \mathrm{S}}^{-}$sind identisch. Im Gegensatz dazu befindet sich die Probe durch einen lateralen Stromfluss im Nicht-Gleichgewicht, und folglich sind auch die quasi-elektrochemischen Potentiale an einem Ort der Probe $\mu_{\mathrm{ec}, \mathrm{P}}^{+}$bzw. $\mu_{\mathrm{ec}, \mathrm{P}}^{-}$nicht identisch. Beim Messprozess mit der Rastertunnelpotentiometrie wird durch anlegen einer Spannung 
das elektrochemische Potential der Spitze derart adjustiert, dass im zeitlichen Mittel kein Tunnelstrom zwischen Spitze und Probe fließt. Gemäß der Definition des lokalen elektrochemischen Potentials befindet sich die Spitze mit dem Teilbereich des stromdurchflossenen Leiters im Gleichgewicht. Im einfachsten Fall ist die Transmission zwischen Spitze und Probe im betrachteten Energieintervall wie auch die Zustandsdichte von Probe und Spitze konstant, und es lässt sich durch die Messvorschrift ein lokales elektrochemisches Potential $\mu_{\mathrm{ec}}(\boldsymbol{r})$ definieren, so dass das ermittelte elektrochemische Potential der Spitze als der Mittelwert

$$
\mu_{\mathrm{ec}, \mathrm{STP}}(\mathbf{r})=\frac{\mu_{\mathrm{ec}, \mathrm{P}}^{+}(\mathbf{r})+\mu_{\mathrm{ec}, \mathrm{P}}^{-}(\mathbf{r})}{2}
$$

der quasi-elektrochemischen Potentiale der Probe interpretiert werden kann. Im Fall von wie in Kapitel 2.1.2.4 beschriebenem diffusivem Ladungstransport in der Probe ist die gemessene elektrochemische Potentialdifferenz der Spitze zwischen zwei Probenorten $\Delta \boldsymbol{r}$ gleich der elektrostatischen Energiedifferenz $e \Delta U$, da das chemische Potential konstant ist, und die elektrostatische Energie dem elektrochemischem Potential folgen kann. Demnach misst man die elektrostatische Potentialdifferenz zwischen zwei Probenorten.

Im ballistischen Transportregime innerhalb der Abschirmlänge ist die Anzahl der Elektronen bzw. das chemische Potential als auch deren energetische Verteilungen nicht trivial beschreibbar. Es kommt zu Änderungen der elektrostatischen Energie - $e \phi$ und dem chemischen Potential. Eine einfache Beschreibung der Messgröße $\mu_{\mathrm{ec}, \mathrm{STP}}$ im Sinne einer elektrostatischen Energie ist dann nicht mehr gegeben.

\subsubsection{STM-Apparatur und technische Realisierung der Rastertunnelpotentiome- trie}

Die hier vorgestellten Experimente wurden an einem in der Arbeitsgruppe entwickelten Tieftemperatur-Rastertunnelmikroskop bei Basisdrücken von $\mathrm{p}<5 \cdot 10^{-11} \mathrm{mbar}$ durchgeführt. Der Aufbau des STM-Kopfes entspricht dem sogenannten Besocke-Typ [32]. Die grobe Positionierung der STM-Spitze erfolgt durch drei segmentierte Röhrenpiezos nach dem Slip-Stick-Verfahren. Der STM-Kopf ist unter einen Helium-Bad-Kryostaten mit einem Fassungsvermögen von 41 montiert. Zusätzlich zur thermischen Kontaktierung sind Silberdrähte am oberen Teil des STM-Kopfes angebracht. Der STM-Kopf sowie HeliumBad-Kryostat wird zusätzlich durch einen Stickstofftank thermisch isoliert. Damit wird an 
der Probenaufnahme eine Temperatur von ungefähr $6 \mathrm{~K}$ erreicht. Die maximal erreichbare Messzeit an einem Ort der Probe beträgt 20 Stunden, bevor der Helium- wie auch Stickstoffkryostat wieder befüllt werden müssen. Drei über UHV-Fenster realisierte optische Zugänge erlauben die genaue Positionierung der STM-Spitze über der Probe sowie beispielsweise eine optische Anregung [33].

Zur elektrischen Kontaktierung der Probe besitzt die Probenaufnahme 6 voneinander isolierte niederohmige elektrische Kontakte, welche jeweils mit einem Strom von bis zu 1,5 A belastet werden können.

Für die Ansteuerung des STM-Kopfes sowie der Datenerfassung steht eine in der Arbeitsgruppe entwickelte Elektronik sowie Messsoftware zur Verfügung. Im Folgenden soll auf die wesentlichen Bestandteile, welche für die Umsetzung von Ladungstransportmessungen mit der Rastertunnelpotentiometrie notwendig sind, eingegangen werden.

Das hier vorgestellte Verfahren der Rastertunnelpotentiometrie wurde von M. A. Schneider entwickelt, von J. Homoth auf eine digitale Ansteuerung erweitert und für Ladungstransportuntersuchungen bei tiefen Temperaturen erstmals in dieser Arbeit eingesetzt.

Eine zusammenfassende, detaillierte Beschreibung und die technische Realisierung ist im Kontext dieser Arbeit entstandenen Veröffentlichung in Review of Scientific Instruments zu finden [34].

Bei dem in dieser Arbeit realisierten Potentiometrie-Verfahren wird das lokale Probenpotential $U_{\text {Trans }}(x, y)$ bestimmt, indem das Potential der Spitze an jedem topographischen Messpunkt $z(x, y)$ so eingeregelt wird, dass $\left\langle I_{\mathrm{T}}\right\rangle=0$ für eine von außen angelegte Probenspannung von $U_{\text {Bias }}=0$ gilt. In Abbildung 11 sind die für die Rastertunnelpotentiometrie wesentlichen Bestandteile schematisch dargestellt. Diese lassen sich in drei Teile unterteilen: der herkömmlichen STM-Elektronik, welche einen zusätzlichen 16-Bit Digital-Analog-Wandler enthält, eine Spannungsquelle zum Aufprägen von lokalen Transportfeldern, realisiert durch zwei weitere 16-Bit DAUs sowie als zentrales Element einen geeigneten Stromverstärker mit einem zusätzlichen ansteuerbaren nicht-invertierten Eingang, welcher sowohl für Standardmessverfahren, wie der Rastertunneltopographie als auch zur Bestimmung der Variationen des lokalen Probenpotentials genutzt wird. Herzstück des Stromver- 
stärkers ist ein rauscharmer Operationsverstärker des Typs AD549 mit einem Rückkopplungswiderstand von $R_{\mathrm{K}} \approx 1 \mathrm{G} \Omega$ und einem daraus resultierenden Verstärkungsfaktor von $\approx 1,2 \cdot 10^{9} \mathrm{~V} / \mathrm{nA}$.

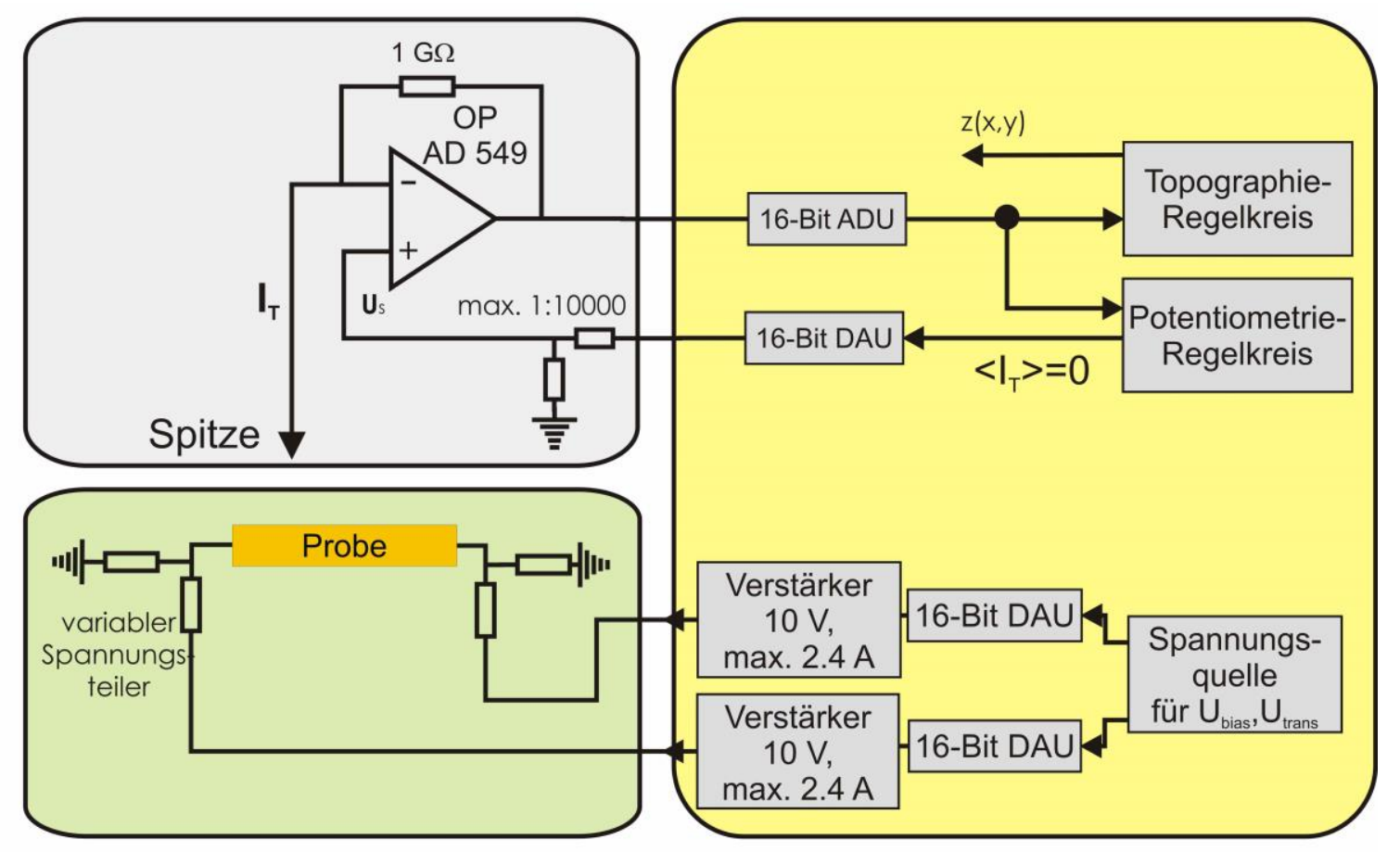

Abbildung 11: Schematische Darstellung der im Vergleich zu herkömmlichen STM-Elektronik wesentlichen Erweiterungen zur Durchführung des in dieser Arbeit verwendeten Rastertunnelpotentiometrie-Verfahrens. Die Abkürzungen DAU und ADU stehen für Digital-Analog bzw. Analog-Digital-Umsetzer.

Das gemessene Stromrauschen der gesamten Elektronik beträgt bei voller Verkabelung und einer Frequenzbandbreite von $1 \mathrm{kHz}$ ungefähr $300 \mathrm{fA}^{3}$. Der invertierte Eingang des Stromverstärkers ist direkt mit der STM-Spitze verbunden. Da beim idealen Operationsverstärker keine Spannungsdifferenz zwischen den Eingängen besteht, kann über den nicht-invertierten Eingang das Kompensationspotential der Spitze gemäß der Potentiometrie-Messvorschrift eingestellt werden. Dies geschieht über einen zusätzlichen 16-Bit DAU mit dahintergeschaltetem Spannungsteiler. Um die Topographie- und Potentiometriedaten zu erhalten, wird ein Zeitmultiplexverfahren verwendet. Bei diesem Verfahren wird an jedem Messpunkt zunächst im ersten Zeitintervall durch einen Proportional-Integral-Regler (PI-

\footnotetext{
${ }^{3}$ Eine Ausführliche Darstellung des frequenzabhängigen Rauschverhaltens der einzelnen im Aufbau verwendeten Komponenten, wie den DAUs und des Stromverstärkers ist in der Dissertation von J. Homoth zu finden [132].
} 
Regler) die Topographie und im zweiten Zeitintervall über den Potentiometrie-Regelkreis, welcher ebenso über einen PI-Regler realisiert ist, das lokale Probenpotential bestimmt.

Die theoretische Auflösungsgrenze dieses Potentiometrieverfahrens wird bei Vernachlässigung aller weiteren Rauschquellen, wie der einzelnen Elektronikbauteile, bei kleinen Tunnelströmen $\left(e\left|R_{\mathrm{T}} I_{\mathrm{T}}\right| \ll k_{B} T\right)$ durch das thermische Spannungs- bzw. auch Johnson-Nyquist-Rauschen des Tunnelkontaktes dominiert [35], welches gegeben ist über

$$
\Delta U=\sqrt{4 k_{\mathrm{B}} T R_{\mathrm{T}} \Delta f}[36,37] .
$$

Um eine hohe Auflösung zu erreichen, ist es nach Gleichung 2.20 daher im Experiment sinnvoll neben tiefen Temperaturen und möglichst kleinen Bandbreiten $\Delta f$ bei niedrigen Tunnelwiderständen zu arbeiten. Dabei muss jedoch beachtet werden, dass mit niedrigeren Tunnelwiderständen ebenso größere Kräfte und eine stärkere elektronische Kopplung zwischen Spitze und Probe herrschen, so dass daher immer ein Kompromiss zwischen einer guten Auflösung und einer nicht invasiven Spitze gefunden werden muss. In Kapitel 4.2 wird anhand von Nullmessungen gezeigt, dass bei Raumtemperatur auf Graphen ein Auflösungsvermögen von nur $30 \%$ oberhalb des theoretisch erwarteten Auflösungsvermögens mit der hier verwendeten Apparatur erreicht werden kann. 


\section{Epitaktisches Graphen auf SiC: Wachstum, strukturelle und elektronische Eigenschaften}

Die strukturellen und elektronischen Eigenschaften von graphitisiertem $\mathrm{SiC}$ werden seit Beginn der 1970er Jahre untersucht. Die Entdeckung der experimentellen Realisierbarkeit von Graphen 2004 mit einigen seiner theoretisch vorhergesagten elektronischen Eigenschaften, wie dem Quantenhall-Effekt, hat zu einem rasanten Anstieg der wissenschaftlichen Untersuchungen von theoretischer als auch experimenteller Seite von Graphen auf verschiedensten Substraten wie auch $\mathrm{SiC}$ geführt. Im folgenden Kapitel der Arbeit sollen die grundlegenden Eigenschaften des Substrats, ein Überblick über bisherige Erkenntnisse zum epitaktischem Wachstum sowie die elektronischen Eigenschaften von Graphen, insbesondere in Bezug auf die $\mathrm{SiC}(0001)$-Oberfläche gegeben werden. Im Anschluss wird eine detaillierte Charakterisierung der strukturellen und elektronischen Eigenschaften der in dieser Arbeit präparierten Proben mit Hilfe der niederenergetischen Elektronenbeugung (engl. Low Energie Electron Diffraction, LEED), der Rasterkraft- und Raster-Kelvin-Mikroskopie sowie Rastertunnelmikroskopie und Spektroskopie gegeben.

\subsection{Eigenschaften des SiC-Substrates}

Siliziumcarbid ( $\mathrm{SiC}$ ) existiert in über 250 verschiedenen kristallinen Formen, den sogenannten Polytypen. Innerhalb des Kristalls liegen die vier Valenzelektronen der Siliziumund Kohlenstoffatome $\mathrm{sp}^{3}$-hybridisiert vor, so dass jedes Atom in der Tetraederstruktur zu seinen nächsten vier Nachbarn gebunden ist, wie in Abbildung 12 (a) dargestellt ist. Eine Ebene der in der Tetraederstruktur gebundenen Silizium- und Kohlenstoffatome bezeichnet man auch als SiC-Doppellagen. Die einzelnen Polytypen unterscheiden sich in der Anordnung und Anzahl der SiC-Doppellagen innerhalb einer Einheitszelle. Si-C Bindungen übereinander liegender Ebenen sind entweder um $60^{\circ} \mathrm{zu}$ den nächsten Nachbarn rotiert und liegen in der hexagonalen oder auch Zinkblende Struktur vor oder sind zueinander gespiegelt in der Wurtzit- bzw. kubischen Struktur. Die kristallographische c-Achse ist dabei definiert als Ebenennormale zu den SiC-Doppellagen, so dass alle Polytypen nach der Stapelfolge entlang der [111]-Richtung fürs kubische Gitter oder entlang der [0001]-Richtung fürs hexagonale Gitter eingeordnet werden können (siehe Abbildung 12 (c)). Die Bezeichnung der verschiedenen Polytypen erfolgt in der Regel nach der Ramsdell-Notation, nach 
der ein SiC-Kristall mit einer Nummer für die Anzahl der SiC-Bilagen innerhalb einer Einheitszelle entlang der c-Achse und dem Buchstaben C, H oder R entsprechend der Kristallstruktur der Einheitszelle kubisch, hexagonal oder rhomboedrisch bezeichnet wird.

a)

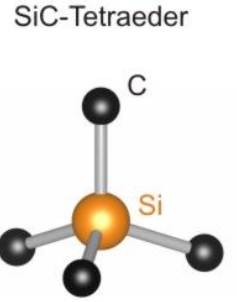

b)

SiC-Doppellage

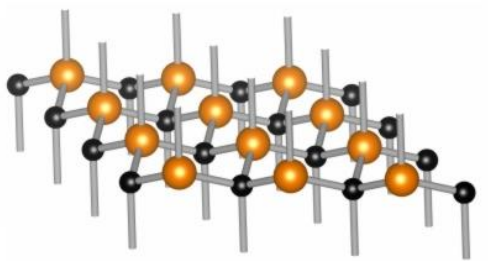

c)
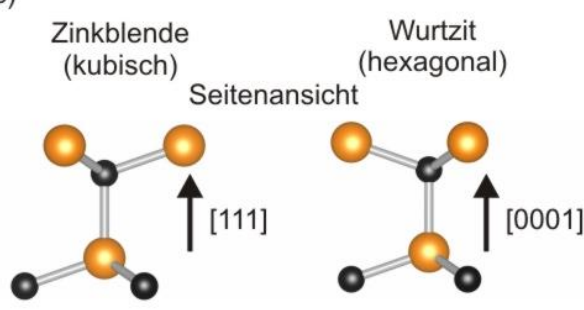

Draufsicht

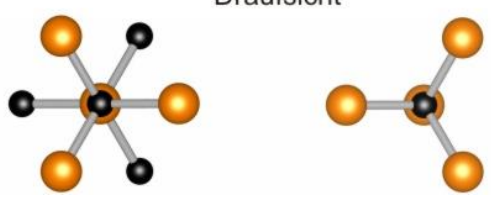

Abbildung 12: (a) SiC Tetraeder mit $\mathrm{sp}^{3}$ hybridisierten Kohlenstoffatomen und Siliziumatom. (b) SiC-Doppellage. (c) Die beiden möglichen Bindungskonfigurationen zweier übereinanderliegender Doppellagen SiC in Seitenan- und Draufsicht für die kubische und hexagonale Struktur. Bei der kubischen Struktur sind die SiC Bindungen benachbarter Doppellagen um $60^{\circ}$ entlang der [111]-Richtung rotiert und bei der hexagonalen Struktur an der (0001)-Ebene gespiegelt.

In Abbildung 13 sind die drei häufig vorkommenden Polytypen 3C, 4H und 6H-SiC(0001) dargestellt. Für das epitaktische Wachstum von Graphen wurden bisher die Polytypen 4H$\mathrm{SiC}(0001)$ und $6 \mathrm{H}-\mathrm{SiC}(0001)$ verwendet. Alle Polytypen des SiC sind Halbleiter mit unterschiedlichsten Bandlücken. Diese erstrecken sich über einen energetischen Bereich von 2,3 eV für rein kubisches $3 \mathrm{C}$ bis 3,6 eV für rein hexagonales $2 \mathrm{H}-\mathrm{SiC}$. 


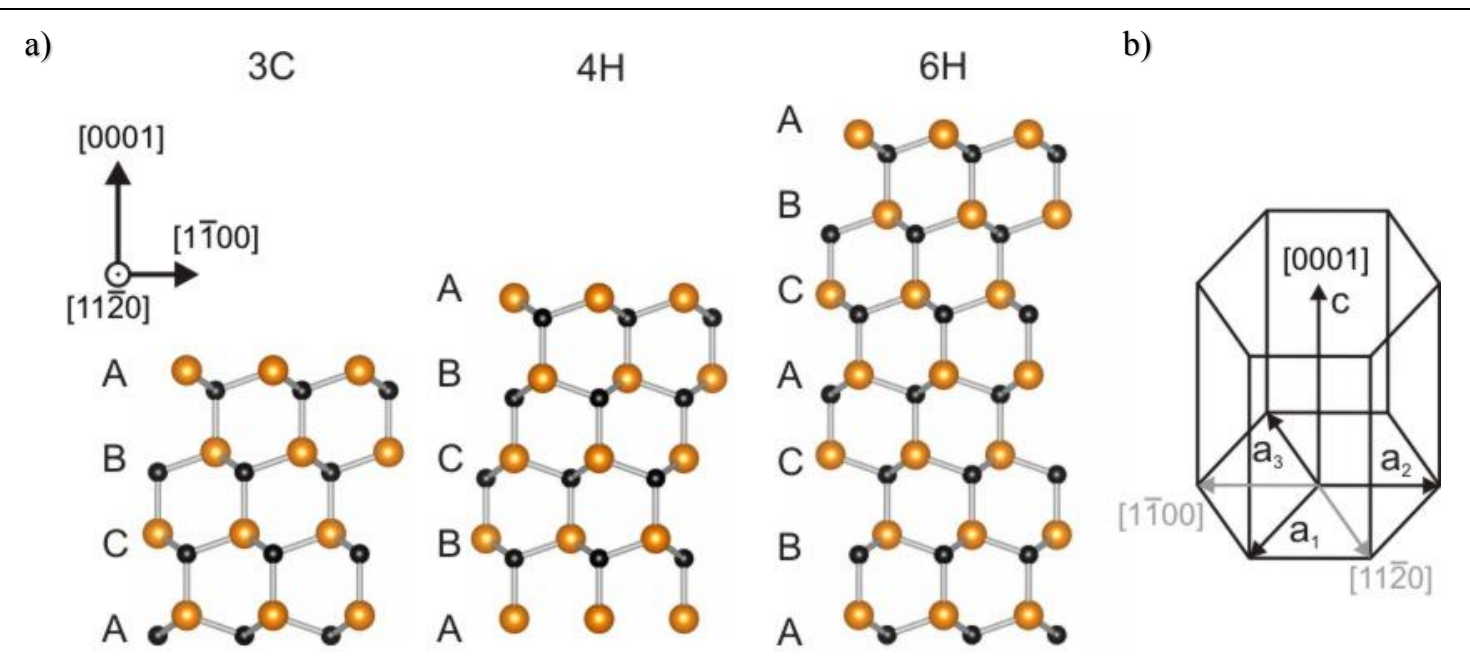

Abbildung 13: (a) Seitenansicht der drei häufigsten SiC Polytypen 3C, 4H und 6H entlang der Richtung [1120]. (b) Für SiC wird im Allgemeinen die hexagonale Kristallnotation mit vier Miller-Indizes verwendet. Die zugehörigen Vektoren $\mathbf{a}_{1}, \mathbf{a}_{2}$ und $\mathbf{a}_{3}$ liegen in einer Ebene, der vierte Vektor $\mathbf{c}$ senkrecht auf dieser. Die oberste Atomlage der (0001) Ebene entlang der [0001]-Richtung ist mit Silizium terminiert, die (0001)-

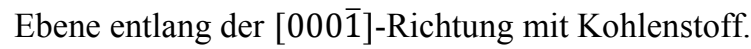

\subsection{Epitaktisches Wachstum von Graphen}

Es gibt drei grundlegende Verfahren epitaktische Graphenfilme auf Halbleitern bzw. Metallen herzustellen, die alle auf der Zersetzung von Carbiden beruhen: die teilweise thermische Zersetzung von $\mathrm{SiC}$, das Kracken von Hydrocarbongas auf Carbiden (z.B. TaC, TiC) oder Metallen wie Nickel oder Platin und die Segregation von Kohlenstoffatomen aus $\mathrm{Ru}(0001)$ [38]. Dabei hat das Verfahren der thermischen Zersetzung von $\mathrm{SiC}$ den großen Vorteil, dass einkristalline Wafer schon über einen geraumen Zeitraum in der Halbleiterindustrie etabliert und daher preiswert und in hoher Qualität vorhanden sind. Das Wachstum von epitaktischem Graphen auf $\mathrm{SiC}$ beruht auf der thermischen Zersetzung des SiC. Beim Erhitzen des Wafers sublimieren aufgrund des im Vergleich zu Kohlenstoff niedrigeren Dampfdruckes des Siliziums Silizium-Atome aus dem Kristall. Aus den zurückbleibenden Kohlenstoffatomen bilden sich die einzelnen Graphenschichten. Betrachtet man die reinen stöchiometrischen Verhältnisse, werden drei SiC-Bilagen benötigt, um eine Lage Graphen zu bilden.

Für das epitaktische Wachstum werden überwiegend die beiden SiC-Polytypen $4 \mathrm{H}$ und $6 \mathrm{H}$ mit den beiden polaren Ebenen (0001) und (0001) verwendet. Das Wachstum sowie die 
elektronischen Eigenschaften der gewachsenen Graphenschichten hängen nicht von der Wahl der verschiedenen Polytypen ab, unterscheiden sich jedoch drastisch von der Wahl der polaren Richtung [0001] bzw. [0001] . Auf der Kohlenstoff-terminierten Oberfläche in

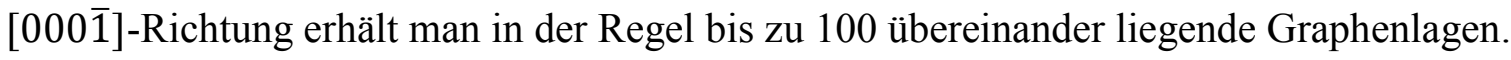
Die Anzahl der Kohlenstofflagen ist auf der Kohlenstoff-terminierten Oberfläche wesentlich stärker von der Wachstumstemperatur und der Zeit abhängig als auf der Silizium-terminierten Oberfläche und daher schwerer zu kontrollieren. Die elektronischen Eigenschaften dieser Filme entsprechen aber denen von einlagigem Graphen, da diese untereinander entkoppelt sind [39].

Im weiteren Verlauf der Arbeit möchte ich mich auf die Beschreibung des Wachstums sowie den elektronischen Eigenschaften von Graphenlagen auf der Si-terminierten Oberfläche beschränken.

Die erste erfolgreiche Graphenpräparation ist van Bommel et al. schon 1975 über die thermische Zersetzung des SiC im UHV gelungen [40]. Bei der Präparation der Graphenlagen auf der Silizium- terminierten Fläche des SiC(0001)-Einkristalls durchläuft die Oberfläche eine Vielzahl von temperaturabhängigen Oberflächenrekonstruktionen wie Anhand von LEED-Untersuchungen gezeigt worden ist [41-43]. Abbildung 14 zeigt den schematischen Präparationsverlauf sowie LEED-Aufnahmen nach den einzelnen Präparationsschritten $[13,41]$. Ausgangspunkt ist das $\mathrm{SiC}(0001)$-Substrat, welches unter einer zusätzlichen Siliziumdeposition von typischerweise $1 \mathrm{ML} / \mathrm{min}$ bei $750^{\circ} \mathrm{C}$ vorbehandelt wird. Die erhaltene Oberfläche hat eine siliziumreiche $3 \times 3$-Rekonstruktion. $\mathrm{Ab} 800^{\circ} \mathrm{C}$ setzt die Siliziumdesorption von der Oberfläche ein [44]. Bei $1000^{\circ} \mathrm{C}$ beobachtet man die $1 \times 1$ Rekonstruktion, die bei weiterer Erhöhung der Temperatur in die $(\sqrt{3} \times \sqrt{3}) \mathrm{R} 30^{\circ}-$ und schließlich in die kohlenstoffreiche $(6 \sqrt{3} \times 6 \sqrt{ } 3)$ R $30^{\circ}$-Oberflächenrekonstruktion übergeht. Diese ist vermutlich größtenteils kovalent zu der darunterliegenden SiC-Lage gebunden (vgl. Kapitel 3.3). Im Temperaturbereich von $1200^{\circ} \mathrm{C}$ bis $1280^{\circ} \mathrm{C}$ setzt die Bildung von Graphen ein, während die Desorption des Siliziums fortschreitet. Wird die Temperatur und/oder die Zeit weiter erhöht, nimmt die Zahl der Graphenlagen zu. Sie bilden eine epitaktisch gewachsene Schicht, wie Anhand der LEED-Aufnahmen in Abbildung 14 zu erkennen ist [13, 41]. 

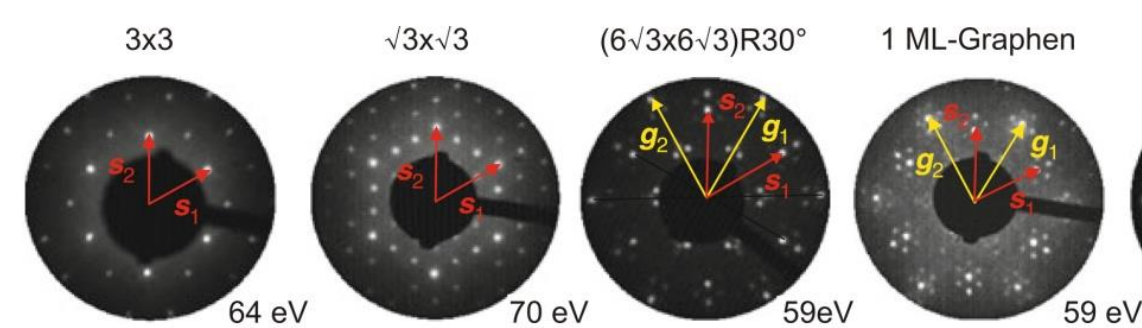

(1x1) Graphite

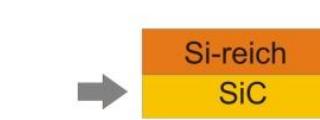

$750^{\circ} \mathrm{C}$

Tempern unter Si-Fluss

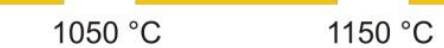

Si-Desorption unter SiC-Zersetzung Si-Fluss

Abbildung 14: Schematischer Präparationsverlauf und LEED-Aufnahmen zu den einzelnen Präparationsschritten nach [41] und in Anlehnung an [13]. Die reziproken Gittervektoren des SiC-Substrates $s_{1}$ und $s_{2}$ sowie die des Graphens $g_{1}$ und $g_{2}$ sind jeweils in den LEED-Aufnahmen eingezeichnet.

Um das Fehlen von Rotationsdomänen auf den gewachsenen Proben zu erklären, haben Emtsev et al. ein inzwischen weitgehend akzeptierten Wachstumsprozess vorgeschlagen, nachdem sich eine neue Graphenlage aus der zuvor an das Substrat gebundenen Zwischenlage bildet und währenddessen vom Substrat aus ein neuer Interface-Layer entsteht [41]. Dieses Szenario wird von Ab-initio-Berechnungen gestützt [45]. Ein weiteres Indiz für dieses Modell ist die Tatsache, dass die oberste Graphenlage kontinuierlich über Substratstufen verläuft, wie atomar aufgelöste STM-Topographien [46] sowie TEM-Untersuchungen gezeigt haben [47].

Aufgrund der hohen Sublimationsrate von Silizium während der Wachstumsphase und Defekt-induzierten unterschiedlichen Desorptionsraten an z.B. Stufenkanten [48] führt die UHV-Wachstumsmethode zu definierten Graphenlagen von maximal einigen $100 \mathrm{~nm}^{2}$, einem inhomogenen Wachstum von ein bis einigen Graphenlagen auf dem Substrat sowie einer Aufrauhung von Substratstufen und Bildung von hexagonalen Löchern (sog. Pits). Abbildung 15 (a) fasst die morphologische Entwicklung der Oberfläche während der Bildung der $\sqrt{3} \times \sqrt{3}$-Rekonstruktion bis hin zur Bildung der dritten Graphenlage zusammen, welche auf AFM-Messungen (Inset Abbildung 15 (a) ii)) und Aufnahmen mit dem niederelektronischen Elektronenmikroskop (LEEM) beruhen [49]. Während die Stufenstruktur bei der Bildung der $\sqrt{3} \times \sqrt{3}$-Rekonstruktion erhalten bleibt, raut die Oberfläche aufgrund der Desorption von Silizium beim Übergang zur $(6 \sqrt{3} \times 6 \sqrt{3})$ R $30^{\circ}$-Rekonstruktion auf, und es bilden sich zahlreiche Terrassen und Stufen. 
a)

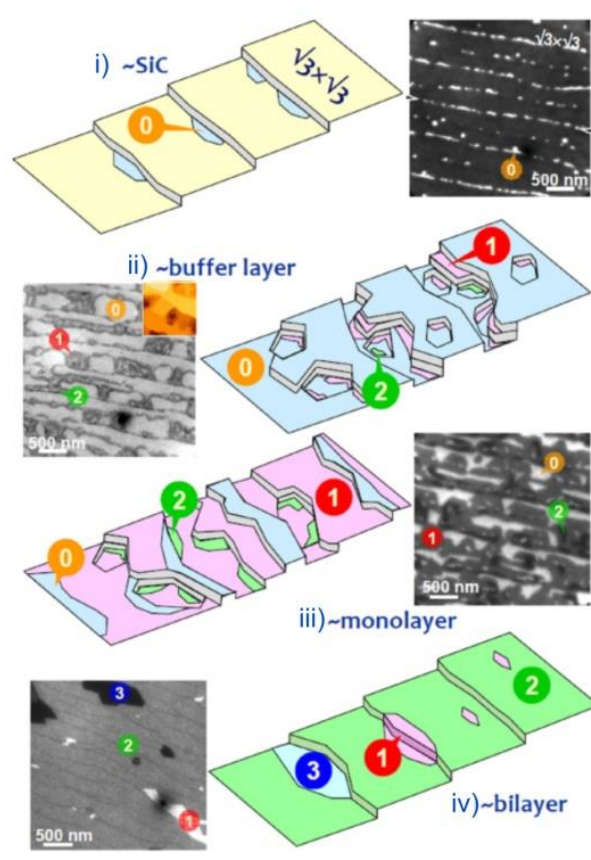

b)

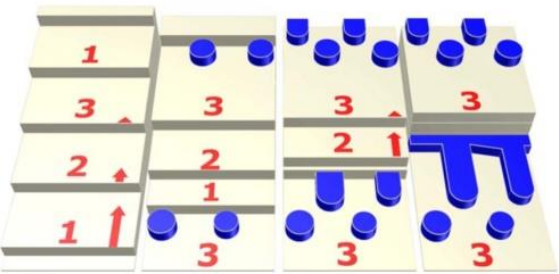

Abbildung 15: (a) Vereinfachte Schematische Darstellung und LEEM-Aufnahmen der SiC-Oberfläche beim Durchlaufen der unterschiedlichen Wachstumsphasen von der i) $\sqrt{3} \times \sqrt{3}$-Rekonstruktion bis zu iv) zweilagigem Graphen nach [49]. Die Anzahl der Lagen ist farblich und nummerisch kodiert. Ein- und zweilagiges Graphen bilden sich vorzugsweise zunächst an Substratstufen. (b) Schematische Darstellung des Wachstumsmodells für zweilagiges Graphen nach [48]. Aufgrund der unterschiedlichen Stapelfolge haben SiC-Substratstufen unterschiedliche Siliziumdesorptionsraten, so dass sich diese mit unterschiedlicher Geschwindigkeit zurückbilden. Daher treten gehäuft dreifache Substrat-Stufenhöhen auf, in deren unmittelbarer Umgebung sich als erstes zweilagiges Graphen bildet.

Die Graphenbildung setzt zunächst an unteren Terrassen der Stufenkanten ein, wobei die mit einlagigem Graphen bedeckte Fläche größer als die Bedeckung von zweilagigem Graphen ist. Mit zunehmender Zeit und weiterer Si-Desorption steigt die Lagenanzahl. Bei einer Bedeckung der Oberfläche mit hauptsächlich zweilagigem Graphen ähnelt die Terrassenstruktur der Oberfläche der zu Präparationsbeginn [49]. Die Bildung von zweilagigem Graphen auf 6H-SiC geht von den SiC-Substratstufen aus, wie von Hupalo et al. beobachtet worden ist. Dabei treten mit zunehmender Zeit vermehrt dreifache SiCSubstratstufen auf, wie es in Abbildung 15 (b) nach [48] dargestellt ist. Es wird in Analogie zur unterschiedlichen Adsorptionsrate von Silizium auf den drei zueinander um $60^{\circ}$ rotierten SiC-Bilagen angenommen, dass die Ursache für das Step-Bunching unterschiedliche Desorptionsraten der drei verschiedenen SiC-Bilagen Oberflächen des 6H-SiC-Polytyps sind. Daraus resultiert eine unterschiedliche Rückbildungsgeschwindigkeit der Stufen, wie 
in Abbildung 15 (b) durch die roten Pfeile dargestellt ist. Um die Silizium-Sublimationsrate von der Oberfläche zu kontrollieren und zu verlangsamen, und damit ein Wachstum mit großflächigen Graphenlagen einheitlicher Schichtdicke zu erreichen, wurden verschiedene Verfahren wie das Wachstum unter Argonatmosphäre [50] oder die CCS-Methode (engl. confiment controlled sublimation) entwickelt [51]. Diese Verfahren ermöglichen das Wachstum von defektfreien Graphenlagen einheitlicher Schichtdicke über mehrere $100 \mu \mathrm{m}^{2}$. Des Weiteren hat neben der Silizium-Desorptionsrate die Morphologie des verwendeten Substrates, wie Stufen und Defektdichte und der verwendete Polytyp einen großen Einfluss auf die Qualität der Graphenfilme [44, 52].

Aufgrund der komplexen Morphologie und des inhomogenen Wachstums der Zwischenschicht sowie der ersten und zweiten Graphenlage von im UHV gewachsenen Proben treten für gewöhnlich unterschiedlichste Stufenhöhen auf den Proben auf. Wählt man die letzte SiC-Schicht als Nullpunkt, wird eine Höhe der Zwischenschicht zwischen 232 pm und 250 pm angenommen $[53,54]$. Die darauffolgenden Graphenschichten liegen nahe am Zwischenschichtabstand von Graphit, welcher 335 pm bei Raumtemperatur beträgt $[55,56]$. Mögliche Stufenhöhen von ein- und zweilagigem Graphen sind in Anlehnung an Filleter in Abbildung 16 mit den entsprechenden Höhenunterschieden zusammengefasst [53].

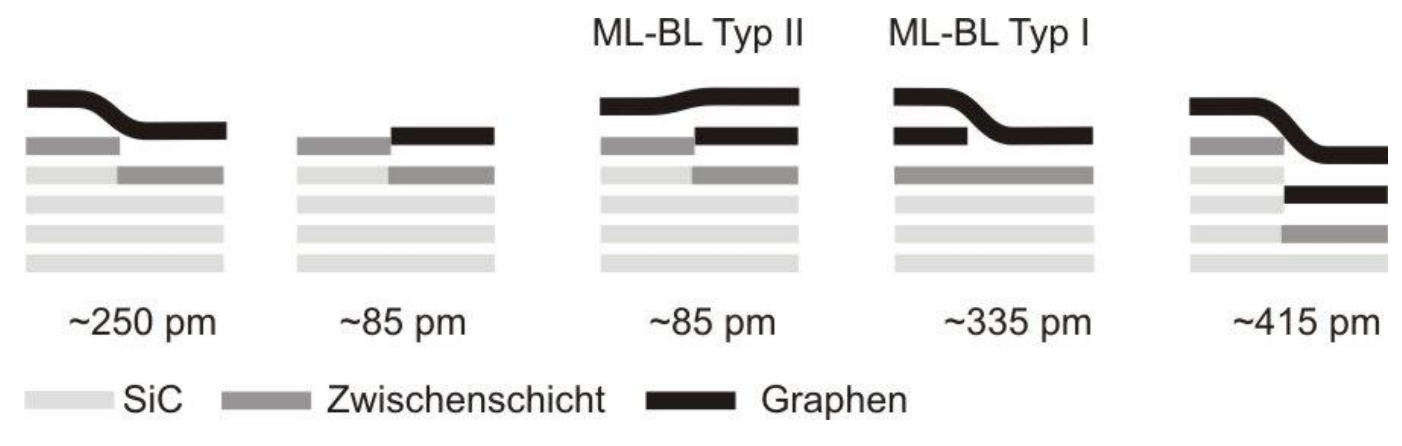

\begin{abstract}
Abbildung 16: Schematische Darstellung an verschiedenen möglichen Kombinationen von SiC-Stufenhöhen, welche mit der Zwischenschicht bzw. einlagigem und zweilagigem Graphen bedeckt sind in Anlehnung an [53]. Das Interface sowie SiC-Bilagen besitzen eine Höhe von 250 pm, ein- und mehrlagiges Graphen eine Höhe von 335 pm. Die oberste Graphenlage verläuft durchgängig.
\end{abstract}

Drei Konfigurationen sind an dieser Stelle besonders hervorzuheben, da diese in Kapitel 5 in Bezug auf den lateralen Ladungstransport näher untersucht werden. Dies ist der Übergang einer Lage Graphen mit darunterliegender Substratstufe sowie der Übergang von einer 
zu zwei Lagen Graphen mit darunterliegender Substratstufe (Typ I) und ohne darunterliegender Substrat-Stufe (Typ II).

\subsection{Strukturelle und elektronische Eigenschaften der $(6 \sqrt{3} \times 6 \sqrt{3}) R 30^{\circ}$ - Rekonstruktion sowie von ein- und zweilagigem epitaktischem Graphen}

Die genaue strukturelle Zusammensetzung der Zwischenschicht von epitaktischem Graphen und dessen Bindungsverhältnisse zum darunterliegenden Substrat werden bis heute sowohl von theoretischer als auch experimenteller Seite kontrovers diskutiert. Dabei werden verschiedene Modelle vorgeschlagen, die sich sowohl in der geometrische Anordnung als auch dem genauen Anteil an Silizium- und Kohlenstoffatomen und deren Bindungsverhältnisse zum Substrat und untereinander unterscheiden [40, 42, 57-61]. Während STMAufnahmen hauptsächlich eine 6×6-Periodizität und nur in seltenen Fällen eine $(6 \sqrt{3} \times 6 \sqrt{ } 3) \mathrm{R} 30^{\circ}$-Struktur zeigen [62], werden mit niederenergetischer Elektronenbeugung ausschließlich Beugungsreflexe einer $(6 \sqrt{3} \times 6 \sqrt{3})$ R $30^{\circ}$-Rekonstruktion beobachtet. Die Angabe der Periodizität bezieht sich dabei auf das SiC-Substrat mit der Gitterkonstanten von $\mathrm{a}_{\mathrm{SiC}}=3,08 \AA$.

Das am meisten verbreitete Modell der Zwischenschicht, welches diesen Widerspruch auflösen kann, ist das eines wie bei Graphen hexagonalen Kohlenstoffgitters, indem ungefähr jedes dritte Kohlenstoffatom kovalent mit einem der darunterliegenden Si-Atome des Substrates gebunden ist. Die $6 \sqrt{3} \times 6 \sqrt{ } 3$ ) R $30^{\circ}$-Einheitszelle beinhaltet dabei 108 SiliziumAtome des darunterliegenden Substrates und 338 Kohlenstoffatome der darüberliegenden Zwischenschicht. Die Einheitszelle der Kohlenstoff-Lage ist dabei um $30^{\circ}$ in Bezug auf die $\mathrm{SiC}\langle 10 \overline{1} 0\rangle$ Richtung rotiert. Um eine möglichst genaue räumliche Übereinstimmung zwischen den Silizium-Atomen des Substrates und den Kohlenstoffatomen der Zwischenschicht zu erhalten, ist die Zwischenschicht leicht verspannt. Die eigentliche Graphenlage liegt über der Zwischenschicht und koppelt nur über Van der Waals-Wechselwirkung mit der darunterliegenden Zwischenschicht.

Abbildung 17 zeigt das theoretische Modell der relaxierten $6 \sqrt{3} \times 6 \sqrt{3})$ R $30^{\circ}$-Rekonstruktion in der Draufsicht und Seitenansicht entlang der [1100]-Richtung nach DFT Berechnungen von [63]. Die Atome der Zwischenschicht oder auch 0-ten Lage sind entlang der [0001]- 
Richtung mit einer $6 \times 6$ Periodizität durchmoduliert. Der Abstand der Kohlenstoffatome zur Substratebene variiert um 89 pm. Bindungen zum Substrat sind nicht eingezeichnet.

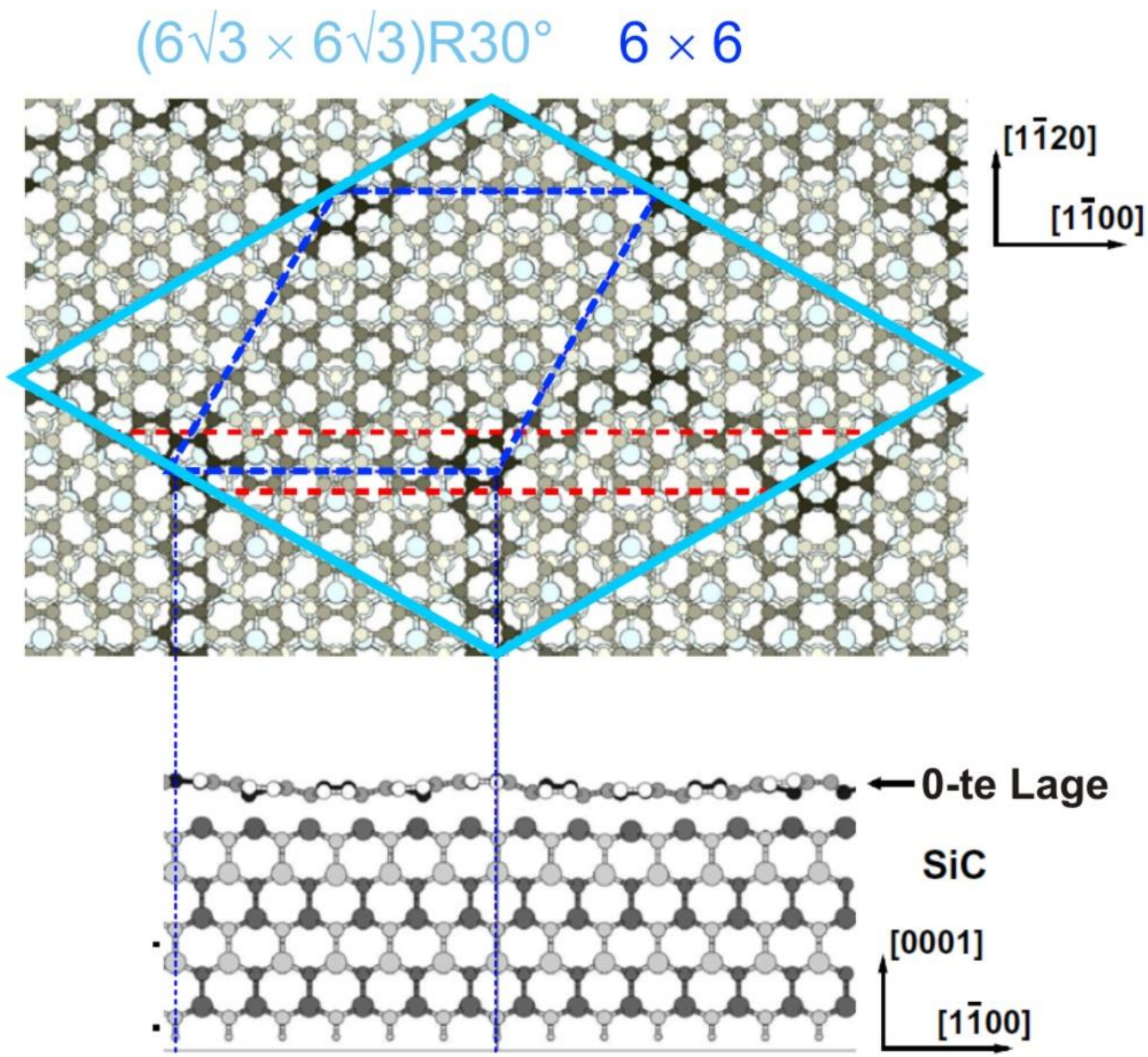

Abbildung 17: Schematische Darstellung der relaxierten $(6 \sqrt{3} \times \sqrt{3})$ R $30^{\circ}$-Rekonstruktion in Draufsicht (oben) und Seitenansicht (unten) mit darunterliegendem Substrat nach [63]. In der Draufsicht sind die Kohlenstoffatome der $(6 \sqrt{3} \times \sqrt{3}) \mathrm{R} 30^{\circ}$-Rekonstruktion (grüne Linie) grau schraffiert, wobei dunklere Atome entlang der [0001]-Richtung höher liegen als heller eingefärbte. Ebenso in blau eingezeichnet ist die quasi- Einheitszelle mit der 6×6-Periodizität. Rot markierter Bereich kennzeichnet die in der Seitenansicht dargestellten Atome der 0-ten Lage, deren Grauwerte entlang der [11̄20]-Richtung zunehmend sind.

Experimentelle Ergebnisse von Core-Level-Spektroskopien weisen darauf hin, dass 9/10 aller Si-Atome des Substrates an jedes dritte Kohlenstoffatom des Interfaces gebunden sind [41]. Gleichzeitig wird angenommen, dass eine bestimmte Anzahl von freien Bindungen zwischen dem Substrat und der Rekonstruktion aufgrund der Gitterfehlanpassung des Graphengitters mit dem SiC-Gitter vorhanden sind. Ein von diesem Modell leicht abgewandeltes ist das von Qi et al. [59], bei dem die 0-te Graphenschicht bzw. die Zwischenschicht 
periodische Einschlüsse von Hexagon-Pentagon-Heptagon $\left(\mathrm{H}_{5,6,7}\right)$ Defekten enthält. Dieses Modell stützt sich ebenso auf STM-Untersuchungen, welche jedoch im Gegensatz zu bisherigen STM-Untersuchungen mit Eisenspitzen durchgeführt worden sind. Jedoch konnte bisher auf der Zwischenschicht kein atomar durchgängiges Kohlenstoffgitter mit Hilfe des STMs für Spannungsbereiche von $-1,5 \mathrm{~V}$ bis $-25 \mathrm{mV}$ beobachtet werden [64]. Außerdem erklären diese beiden Modelle nur unzureichend die topographische Struktur der Zwischenschicht ohne darüberliegender Graphenschicht, welche von zahlreichen Gruppen beobachtet wird [60,65]. Abbildung 18 (a) zeigt eine typische STM-Aufnahme der Zwischenschicht ohne darüberliegende Graphenschicht von Hu et al. [65]. Auf der Oberfläche sitzen zahlreiche dreieckige Strukturen bzw. Cluster (grün markiert), die zueinander in einer 6×6Periodizität angeordnet sind.
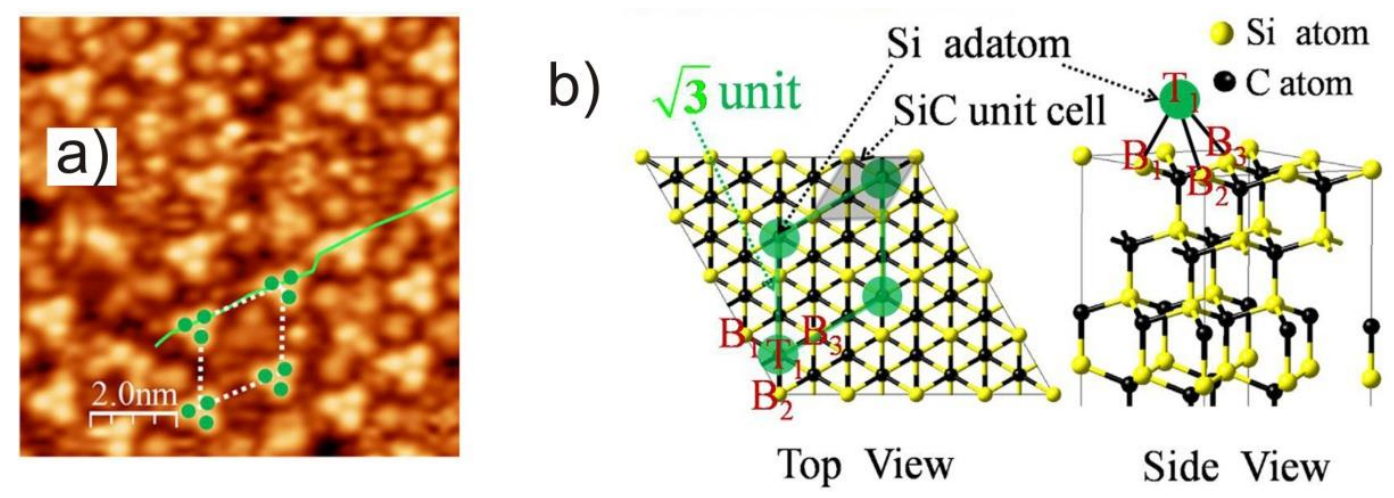

Abbildung 18: (a) STM-Topographie der Zwischenschicht und (b) dazugehöriges Strukturmodell nach Hu et al. $\left(U_{\text {Bias }}=1,7 \mathrm{~V}, I_{\mathrm{T}}=176 \mathrm{pA}\right)[65]$. Die Topographie zeigt Trimere durch grüne Punkte markiert, welche sich auf Gitterplätzen der 6×6-Rekonstruktion anordnen. (b) Drauf- und Seitenansicht des SiC-Kristalls. Grüne Kreise markieren mögliche Adatompositionen innerhalb eines Trimers. Jedes Silizium-Atom ist an drei darunterliegende Silizium-Atome kovalent an Positionen $\operatorname{der}(\sqrt{3} \times \sqrt{3})$ R30 $30^{\circ}$-Rekonstruktion zu finden.

Es wird vermutet, dass jedes der Trimere sich aus drei Si-Adatomen zusammensetzt, welche an der $\mathrm{T}_{1}$ Position, wie in Abbildung 18 (b) dargestellt, jeweils an drei Si-Atome des darunterliegenden SiC-Substrates gebunden sind. Somit ist jeweils ein Danglingbond je Adatom vorhanden. Die einzelnen Si-Adatome liegen wiederum auf Gitterplätzen der $(\sqrt{3} \times \sqrt{3}) R 30^{\circ}$-Rekonstruktion des SiC-Substrates, wie schematisch in Abbildung 18 (b) gezeigt. Die Zwischenräume sollen nicht näher spezifizierten amorphen Kohlenstoff enthalten, welcher als Quelle für die Graphenbildung dient. 
Um die elektronischen Eigenschaften der $(6 \sqrt{3} \times 6 \sqrt{3})$ R $30^{\circ}$-Rekonstruktion sowie der darüberliegenden Graphenschichten zu analysieren, wurden zahlreiche Photoemissionsstudien durchgeführt. Abbildung 19 zeigt die mit Hilfe der winkelaufgelösten Photoemission (engl. ARPES) aufgenommene Valenzbandstruktur der $(6 \sqrt{3} \times 6 \sqrt{3})$ R $30^{\circ}$-Rekonstruktion und der von einlagigem Graphen nach [41]. Beide Spektren weisen s-Bänder auf, so dass davon ausgegangen wird, dass die Anordnung der Kohlenstoffatome in der $(6 \sqrt{3} \times 6 \sqrt{3}) \mathrm{R} 30^{\circ}$-Rekonstruktion identisch zu der in der darüberliegenden Graphenlage ist. Im Gegensatz zum gut erkennbaren $\pi$-Band auf Graphen, ist dieses auf der $(6 \sqrt{3} \times 6 \sqrt{3}) \mathrm{R} 30^{\circ}$-Rekonstruktion nahezu nicht vorhanden. Gleichzeitig sind neben den lokalisierten Zuständen $\mathrm{g}_{1}$ und $\mathrm{g}_{2}$ bei 0,5 eV und 1,6 eV an der Fermi-Energie keine weiteren Zustände sichtbar. Dies spricht für ein nicht-metallisches Interface $[19,41]$.

(a) $(6 \sqrt{ } 3 \times 6 \sqrt{ } 3)$

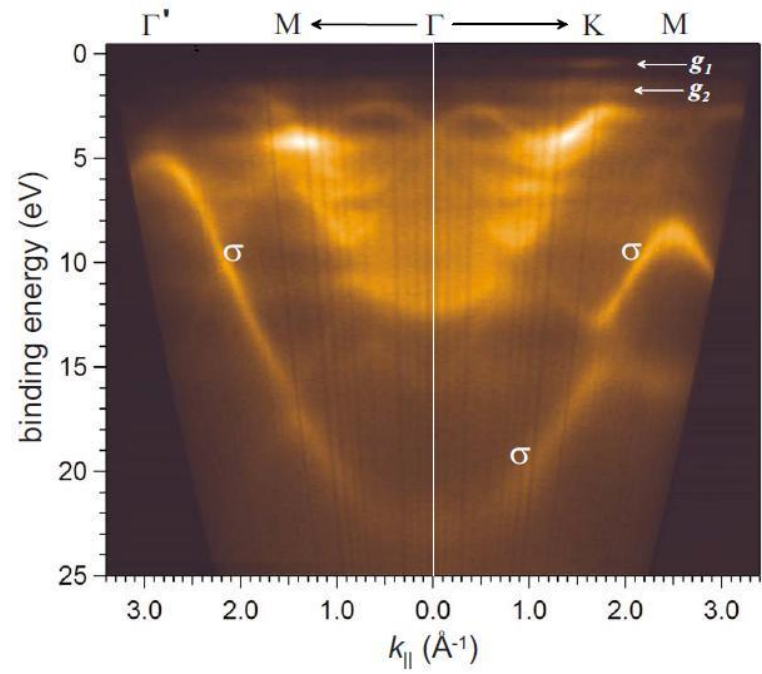

(b) graphene

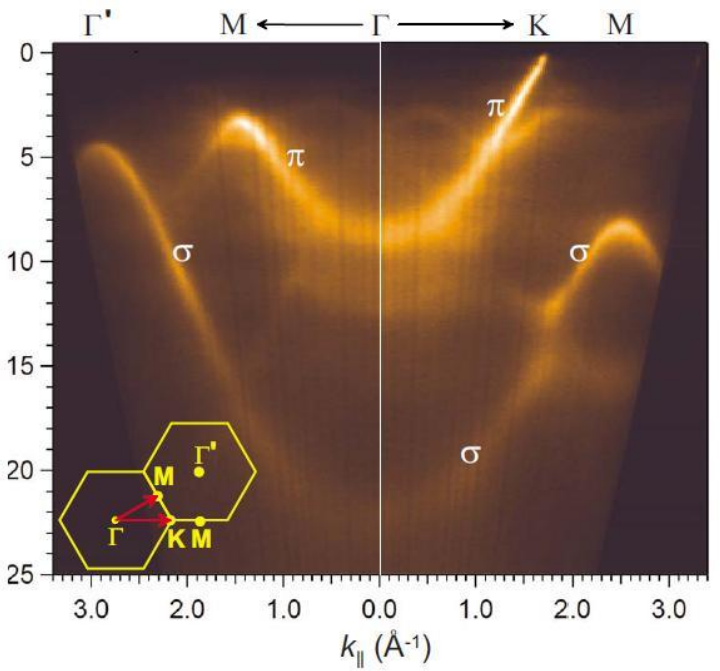

Abbildung 19: Photoemissionsdaten der Zwischenschicht (a) und einlagigen Graphens (b) in Abhängigkeit der Bindungsenergie und der $\mathrm{k}_{\|}$- Komponente des Elektronenimpulses entlang der ГКМ- und ГМГ-Richtung nach [41]. Die Zwischenschicht wie auch die einlagige Graphenschicht zeigen die für Graphen typischen $\sigma-$ Bänder zwischen 5,1 und 22,7 eV. Bis auf lokalisierte Zustände $\mathrm{g}_{1}$ und $\mathrm{g}_{2}$ bei 0,5 eV und 1,6 eV sind keine Zustände nahe $E_{\mathrm{F}}$ bei der somit nicht-metallischen Zwischenschicht sichtbar.

Höher aufgelöste ARPES-Messungen der Valenzbänder um den K-Punkt von ein- und zweilagigem Graphen sind in Abbildung 20 (a) bzw. 11 (b) von Ohta et al. zu sehen [19]. 


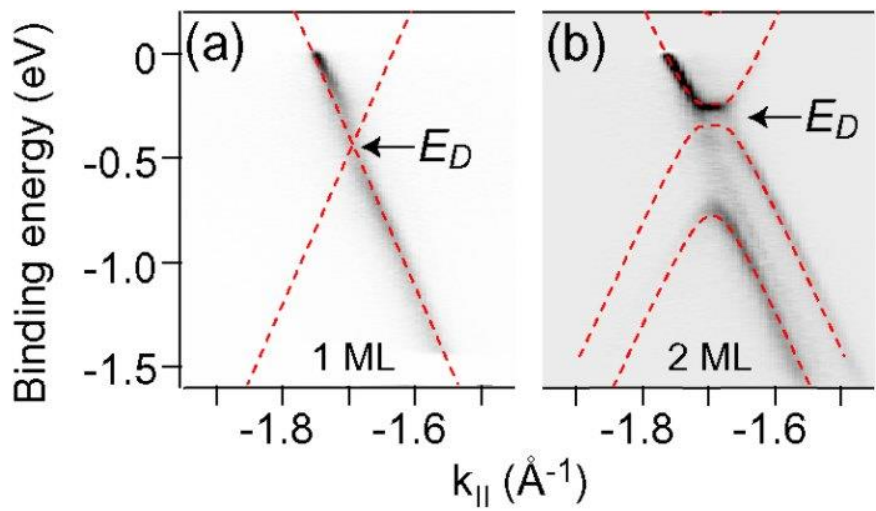

(c)

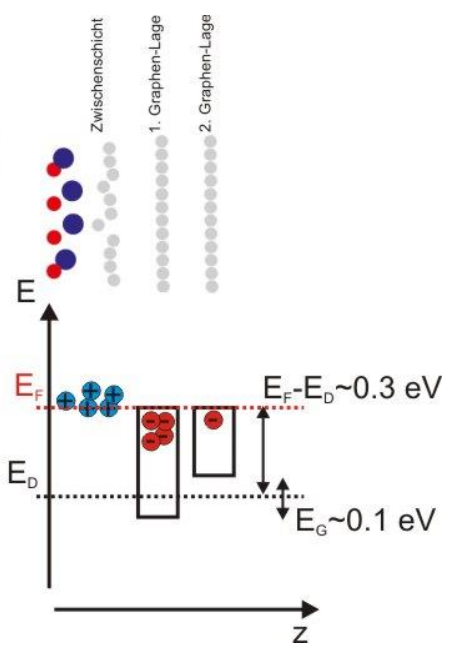

Abbildung 20: ARPES-Messungen nach [19] der Valenzbänder von ein- und zweilagigem Graphen entlang der Symmetriepunkte M-K- $\Gamma$ der Brillouin-Zone $\left(\mathrm{k}_{\|, \Gamma}=0 \AA^{-1}, \mathrm{k}_{\|, \mathrm{K}}=-1,703 \AA^{-1}, \mathrm{k}_{\|, \mathrm{M}}=-2,555 \AA^{-1}\right)$. Die Bänder lassen sich gut durch das Tight-Binding-Modell für ein- und zweilagiges Graphen beschreiben (rot gestrichelte Linien). Beide Lagen sind n-dotiert, für einlagiges Graphen liegt $E_{\mathrm{D}} \approx-0,44 \mathrm{eV}$, für zweilagiges Graphen bei $E_{\mathrm{D}} \approx-0,3 \mathrm{eV}$. (c) Schematische Darstellung der räumlichen Ladungsverteilung auf zweilagigem epitaktischem Graphen in Anlehnung an [66, 67].

Sowohl ein- als auch zweilagiges Graphen zeigt ein lineares bzw. parabolisches Verhalten nahe $E_{\mathrm{D}}$, wie man es nach dem Tight-Binding-Ansatz erwartet. Abweichungen vom linearen Spektrum des einlagigen Graphens werden auf Wechselwirkungen der Elektronen untereinander sowie mit Phononen und Plasmonen zurückgeführt [68]. Der Dirac-Punkt liegt für einlagiges Graphen bei $-0,44 \mathrm{eV}$ unterhalb von $\mathrm{E}_{\mathrm{F}}$ und für zweilagiges Graphen bei $0,3 \mathrm{eV}$. Im Vergleich zu freistehendem Graphen hat epitaktisches Graphen eine höhere Elektronenkonzentration. Die Elektronendichte ist für ein- und zweilagiges Graphen nahezu identisch und hängt nicht von der Lagenanzahl ab. Die Dotierung der Graphenlagen mit Elektronen stammt nicht wie zunächst vermutet von Dotieratomen des Substrates sondern kann durch Donatorzustände der Zwischenschicht erklärt werden [67, 69]. Dabei findet ein Ladungstransfer von Elektronen der Zwischenschicht in die Graphenlagen statt. Analog zu anderen zweidimensionalen Elektronengasen wie der $\operatorname{Si}(111)-\sqrt{3} \times \sqrt{3}) \mathrm{R} 30^{\circ}$-Silber Oberflächenrekonstruktion kann dabei die Dotierung der obersten Graphenlage durch zusätzliches Aufbringen von z.B. Kalium auf die Graphenoberfläche beeinflusst werden [66]. Die auf zweilagigem Graphen beobachtete Bandlücke von ungefähr $E_{\mathrm{G}} \approx 110 \mathrm{meV}$ wird durch die ungleiche Ladungsverteilung zwischen der ersten und zweiten Graphenlage verursacht. Die Ladungsträgerkonzentration der ersten Lage liegt um eine Größenordnung 
über der der zweiten Kohlenstofflage, so dass die beiden übereinanderliegenden Graphenlagen eine Potentialdifferenz $\left|E_{1}-E_{2}\right| \approx 110 \mathrm{meV}$ besitzen. Dies ist im Inset von Abbildung 20 (c) in Anlehnung an $[66,67]$ dargestellt.

\subsection{Probenpräparation und Charakterisierung}

Zur Herstellung von ein- bis zweilagigem Graphen wurde ein mit Stickstoff dotierter 2Zoll SiC(0001)-Wafer der Firma Cree mit einer spezifizierten Dicke von 230 bis $280 \mu \mathrm{m}$ verwendet. Der spezifische Widerstand beträgt $0,062 \Omega \mathrm{cm}$ und die vom Hersteller angegebene Fehlneigung ist kleiner als $0,05^{\circ}$. Die Silizium-Seite ist zusätzlich vom Hersteller chemisch poliert worden, um eine möglichst defektfreie Oberfläche zu erhalten. Abbildung 21 (a) zeigt eine $2 \times 2 \mu \mathrm{m}^{2}$ große AFM-Topographie und in Abbildung 21 (b) das simultan dazu aufgenommene Phasenbild.

(a)

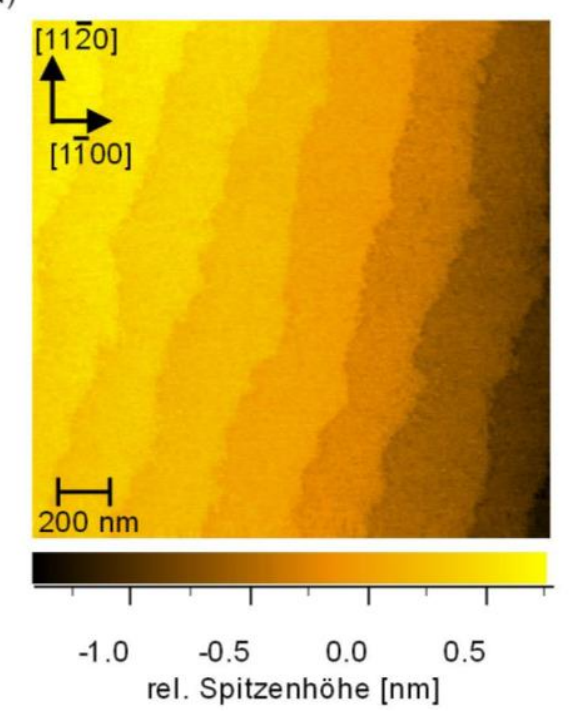

(b)

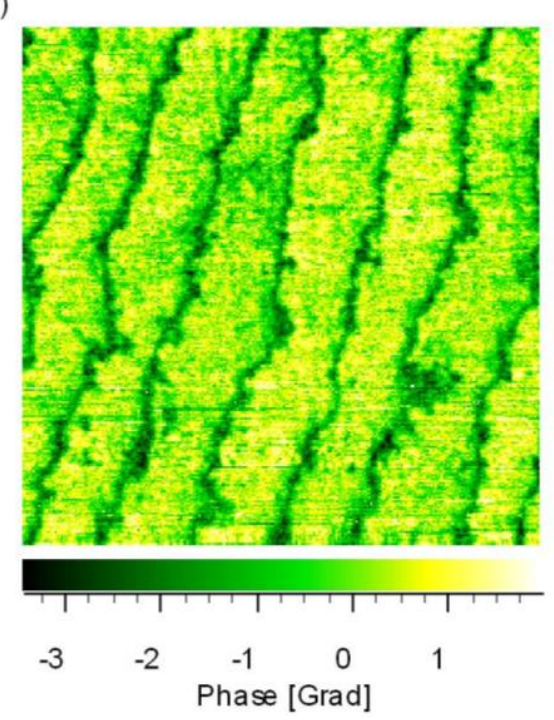

Abbildung 21: (a) $2 \times 2 \mu \mathrm{m}^{2}$ große AFM-Topographie des für die Präparation verwendeten SiC(0001) Substrates. Die Stufenkanten der einzelnen ca. $200 \mathrm{~nm}$ breiten Terrassen verlaufen nahezu parallel entlang der [11̄̄0]. (b) Das Phasenbild der AFM-Topographie zeigt keine nennenswerten Kontraste auf den Terrassen.

Die Oberfläche zeigt atomar glatte Terrassen von ca. $200 \mathrm{~nm}$ Breite. Bei den Stufen handelt es sich um monoatomare Doppellagen der SiC(0001)-Oberfläche. Die mittlere Stufenhöhe in Abbildung 21 (a) beträgt 261(20) pm und stimmt im Rahmen der Messgenauigkeit des 
AFMs gut mit der theoretischen Stufenhöhe von 0,25 nm überein. Das dazugehörige Phasenbild zeigt keine besonderen Merkmale.

Um für die Präparation geeignete Probengrößen zu haben, wurden aus dem Wafer Probenstücke der Größe $2 \times 8 \mathrm{~mm}^{2}$ herausgebrochen. Dazu wurden mit Hilfe einer Diamantritznadel entlang der [11 $\overline{2} 0]$ - und [11̄00]-Richtung Ritze gesetzt und Probenstücke der entsprechenden Größe aus dem Wafer gespalten.

Abbildung 22 zeigt den Aufbau des für die Graphenpräparation entwickelten Probenhalters. Das Probenstück liegt über zwei Tantalauflagen und wird über zwei darüberliegenden Tantalblechen auf einer Fläche von 0,5 bis 1,5 mm mit Hilfe von Kuper-Nickel Schrauben eingeklemmt und gleichzeitig elektrisch kontaktiert. Da sowohl die Bleche als auch der Wafer oxidiert sind, beträgt der Gesamtwiderstand vor Präparationsbeginn einige $\mathrm{k} \Omega$.

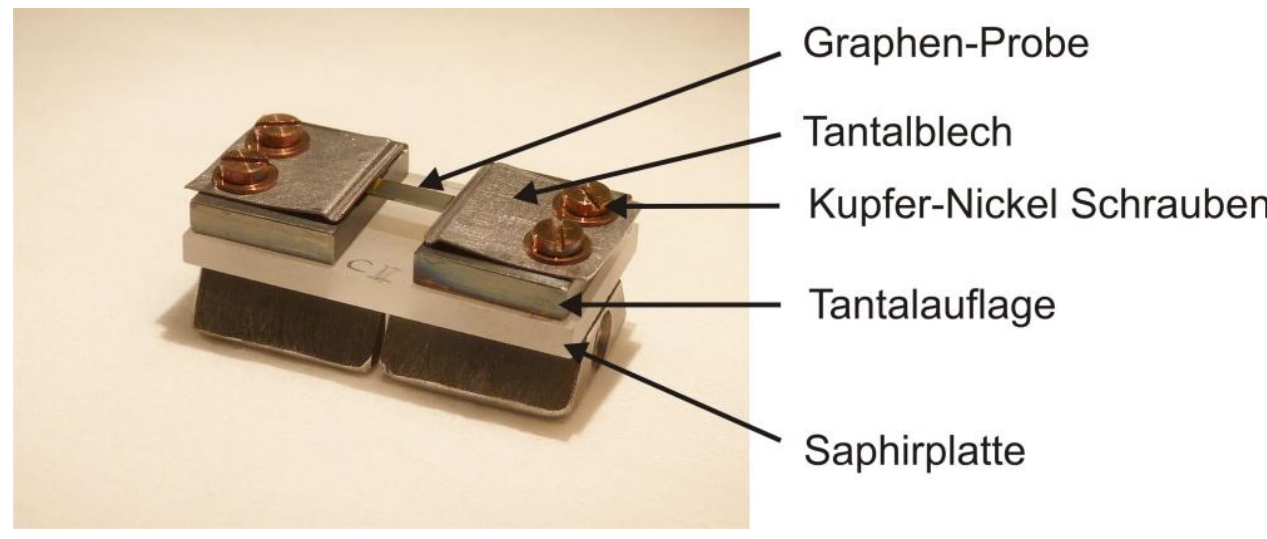

Abbildung 22: Probenhalter mit eingebauter Grapen-Probe zur Präparation und Untersuchung im STM, einsetzbar für Probentemperaturen zwischen $6 \mathrm{~K}$ und $1800 \mathrm{~K}$.

Die eigentliche Graphenpräparation findet unter UHV-Bedingungen bei Basisdrücken von $3 \cdot 10^{-11}$ mbar statt. Dazu wird die Probe über die Kontakte zunächst über 6 bis 12 Stunden resistiv zwischen $500{ }^{\circ} \mathrm{C}$ und $600{ }^{\circ} \mathrm{C}$ geheizt, um Verunreinigungen vom Probenhalter als auch von der SiC-Oberfläche zu entfernen. Im nächsten Schritt wird die Temperatur in mehreren Zyklen innerhalb weniger Sekunden von $600{ }^{\circ} \mathrm{C}$ auf $1450{ }^{\circ} \mathrm{C}$ erhöht. Die steilen Temperaturrampen sollen ein Aufrauen der Oberfläche durch eine zu starke Siliziumdesorption vor der eigentlichen Graphenbildung verhindern [48]. Währenddessen ist gleichzeitig ein Druckanstieg in der Präparationskammer zu verzeichnen, der zunächst 
durch die Oxiddesorption von der SiC-Oberfläche als auch durch die anschließende Siliziumdesorption verursacht wird. Gleichzeitig erhitzen sich auch weitere Komponenten der Präparationsanlage. Daher wird die eigentliche Wachstumstemperatur für 10 bis $60 \mathrm{~s}$ gehalten und die Wachstumsphase bei Kammerdrücken oberhalb von $1 \cdot 10^{-9}$ mbar abgebrochen und die Substrattemperatur wider unterhalb $600{ }^{\circ} \mathrm{C}$ gebracht, um eine zusätzliche Kontamination durch warm werdende Anlagenteile zu verhindern. Diese Heizzyklen werden fünf- bis zehnmal wiederholt. Durch die einzelnen Heizschritte diffundiert ebenso Tantal in das Siliziumcarbid und bildet stabile ohmsche Kontakte bei Raumtemperatur bis hin zu mehreren $100{ }^{\circ} \mathrm{C}$ [47], so dass der Probenwiderstand nach der Präparation bei RT in der Größenordnung von einigen Ohm liegt.

Die Qualität der präparierten Graphenfilme wird im Anschluss der Präparation mit der niederenergetischen Elektronenbeugung (engl. Low Energy Electron Diffraction, LEED) überprüft. Abbildung 23 (a) zeigt die Beugungsreflexe der unpräparierten Probe vor den Heizschritten. Die LEED-Aufnahme zeigt die typischen SiC 1×1-Reflexe.

(a)

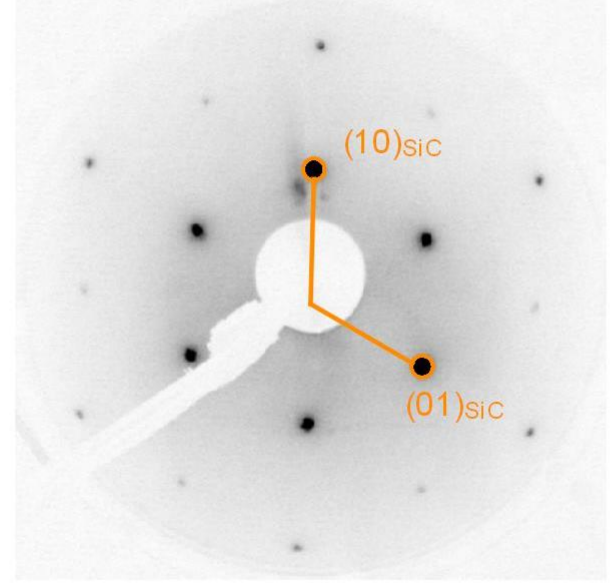

(b)

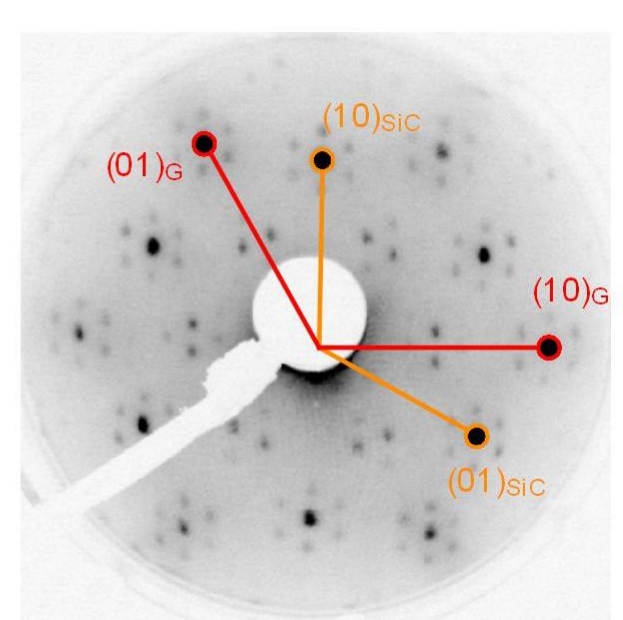

Abbildung 23: (a) LEED-Aufnahme des SiC(0001)-Substrats bei einer kinetischen Energie von $171 \mathrm{eV}$, (b) LEED-Aufnahme einer Graphenprobe mit einer Bedeckung von ein bis zwei Lagen, aufgenommen bei 108 eV. Die Präparationstemperatur betrug $1450{ }^{\circ} \mathrm{C}$ für 4 min. Beugungsspots erster Ordnung des SiC-Gitters sind orange und des Graphengitters rot gekennzeichnet.

Diese haben eine sechszählige Symmetrie, da aufgrund der Stapelfolge auf der Oberfläche die drei zueinander um die c-Achse um $60^{\circ}$ rotierten SiC-Doppellagen auftreten können 
[70]. Nach einer erfolgreichen Präparation erhält man neben den SiC 1×1-Spots eine Vielzahl zusätzlicher Beugungsreflexe, wie in Abbildung 23 (b) zu sehen ist. Die Graphen $1 \times 1$ Spots sind um $30^{\circ} \mathrm{zu}$ den $\mathrm{SiC} 1 \times 1-$ Reflexen rotiert und fallen mit Positionen der im reziproken Raum kleinsten Einheitszelle der $(6 \sqrt{3} \times 6 \sqrt{ } 3)$ R30 $30^{\circ}$-Überstruktur zusammen. Die Position aller weiteren Spots lässt sich als Linearkombination der reziproken Einheitsvektoren des Substrates und der $(6 \sqrt{ } 3 \times 6 \sqrt{ } 3) \mathrm{R} 30^{\circ}$-Überstruktur darstellen und haben in der Regel eine geringere Intensität [42]. Die Intensität der einzelnen Spots ist stark energieabhängig. Eine Unterscheidung zwischen der Zwischenschicht sowie ein- und zweilagigem Graphen kann prinzipiell Anhand von relativen Intensitätsvergleichen bestimmter Spots mit Hilfe der niederenergetischen Elektronenbeugung vorgenommen werden [71], setzt aber eine genaue Positionierung der Proben bezüglich des auftreffenden Elektronenstrahls voraus.

Eine für die Transportmessung wichtige niederohmige elektrische Kontaktierung der Graphenschicht bei tiefen Temperaturen konnte aber über diese Kontaktierung nicht realisiert werden. Daher wurden die Proben nach der Präparation ausgeschleust und unter Hochvakuumbedingungen über eine Schattenmaske Goldkontakte in einem Abstand von $2 \mathrm{~mm}$ und einer Dicke von 15 bis $50 \mathrm{~nm}$ auf die Graphenschicht aufgedampft. Diese Art der Kontaktierung ermöglicht ohmsche Kontakte im Temperaturbereich von $6 \mathrm{~K}$ bis Raumtemperatur. Die Stabilität der elektrischen Kontakte auch für höhere Temperaturen ist sehr wichtig, da es nötig ist, die Graphenproben vor den STM-Untersuchungen im UHV noch einmal auf bis $600{ }^{\circ} \mathrm{C} \mathrm{zu}$ heizen, um Verunreinigungen von der Oberfläche $\mathrm{zu}$ beseitigen. Abbildung 24 zeigt I(U)-Kurven ein- und derselben Probe, welche zwischenzeitlich für mehrere Wochen ex situ gelagert und anschließend bei $350-600{ }^{\circ} \mathrm{C}$ für $5 \mathrm{~h}$ im UHV gesäubert wurde. Die I(U)-Kurven wurden bei 6 K direkt im STM über eine Zweipol-Messung an den Kontakten bestimmt. Der Verlauf der Kurven ist linear und für beide Polaritäten symmetrisch. Daraus lässt sich ein Gesamtwiderstand der Probe nach der ersten Messung zu (1259 \pm 25$) \Omega$ und der zweiten zu (1252 \pm 25$) \Omega$ bestimmen. Der Fehler wurde hier mit $2 \%$ abgeschätzt. Die bestimmten Widerstandswerte unterscheiden sich nur geringfügig. Anhand der elektrischen Charakterisierung kann man daher davon ausgehen, dass das Aussetzen der Proben an Luft und das erneute notwendige Tempern die Qualität dieser nicht wesentlich beeinträchtigt. Mit den Kontaktabständen von 4,2(1) mm und einer Breite von 1,8(1) mm der Probe erhält man einen makroskopischen Flächenwiderstand der epitaktisch gewachsenen 


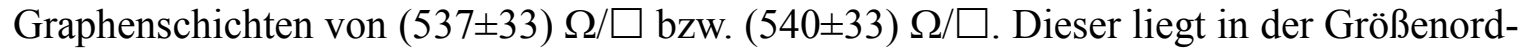
nung bisheriger Transportstudien, welche in Kapitel 5 im Zusammenhang mit eigenen mikroskopischen Transportmessungen vorgestellt werden.

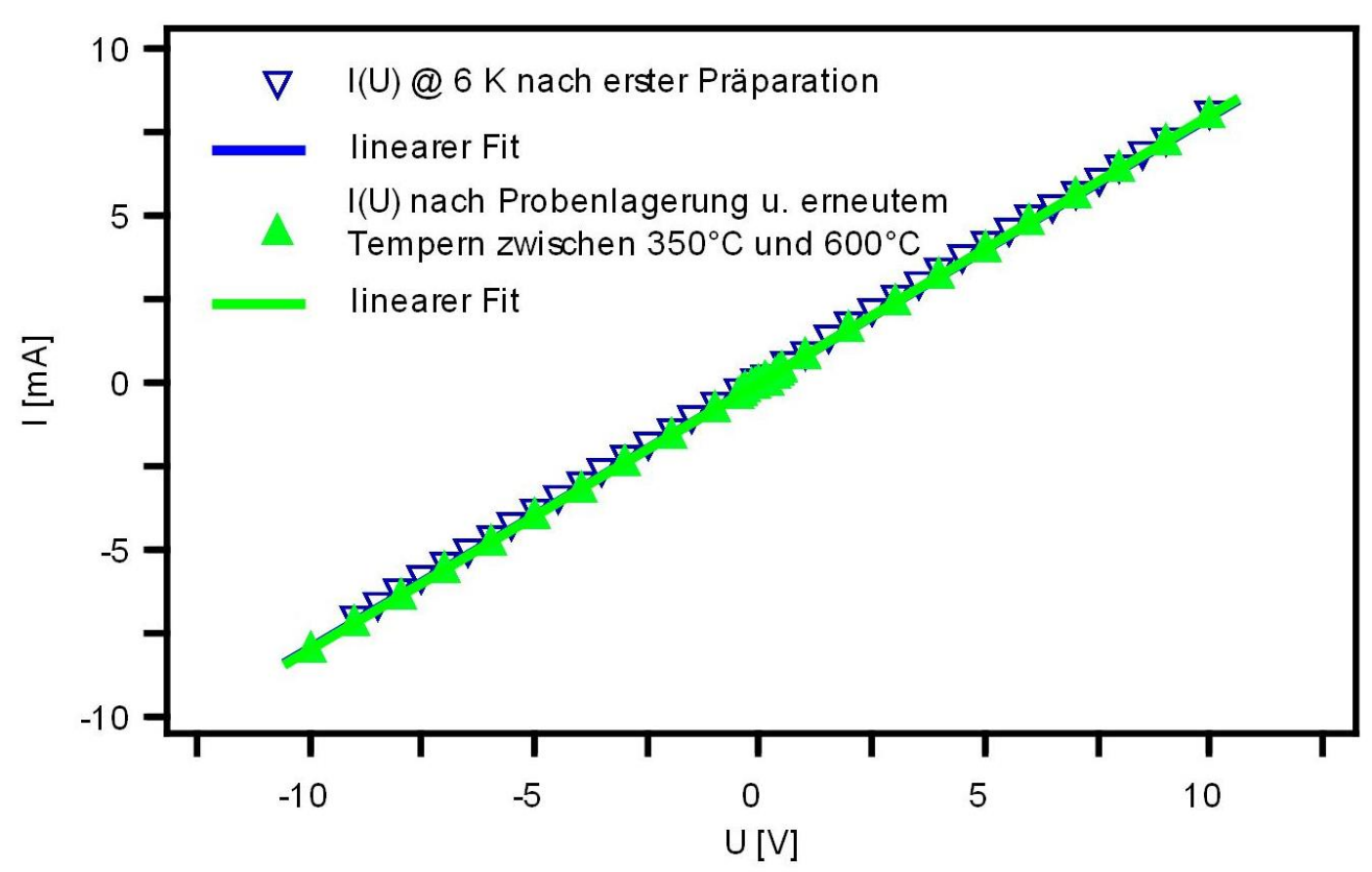

Abbildung 24: Im UHV bei 5,6 K gemessene I(U)-Kennlinien einer fertig präparierten SiC(0001)-Graphen Probe unmittelbar nach der erster Präparation (blau) sowie nach Lagern an Atmosphäre, erneutem Einschleusen ins UHV sowie Tempern zwischen $350-600{ }^{\circ} \mathrm{C}$ (grün). Die I(U)-Kennlinien verlaufen linear und symmetrisch um den Nullpunkt. Der Probenwiderstand zeigt im Rahmen des Messfehlers von 2 \% keine signifikante Widerstandsänderung. Er beträgt im Mittel (1256 \pm 36$) \Omega$.

Wie in Kapitel 5.1 mit Hilfe der Raster-Kelvin-Mikroskopie gezeigt wird, tragen die Kontakte keinen signifikanten Beitrag zum Gesamtwiderstand bei. Ebenso ist der Beitrag des Substrates vernachlässigbar, da die Ladungsträger des SiC-Substrates zu tiefen Temperaturen hin ausfrieren und damit der Widerstand des Substrates bei $6 \mathrm{~K}>1 \mathrm{M} \Omega$ wird [72].

Eine topographische Analyse der präparierten Proben erfolgt anschließend mit dem AFM ex situ. Abbildung 25 (a) zeigt einen $4 \times 4 \mu \mathrm{m}^{2}$ großen Bereich einer für 2 min. bei max. $1600{ }^{\circ} \mathrm{C}$ geheizten Probe und das simultan dazu aufgenommene Phasenbild (Abbildung 25 (b)). Die Morphologie hat sich im Vergleich zum nicht präparierten Substrat deutlich verändert. Die Fläche atomar glatter Bereiche hat zugenommen und die Kontur der Stufen hat sich auf der Längenskala von einigen $100 \mathrm{~nm}$ aufgeraut. 
(a)

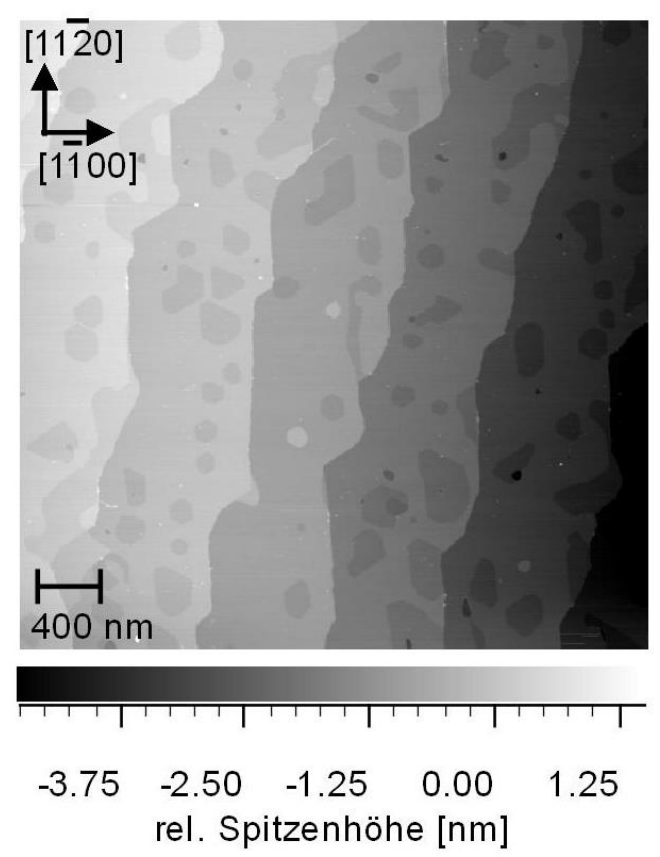

(b)

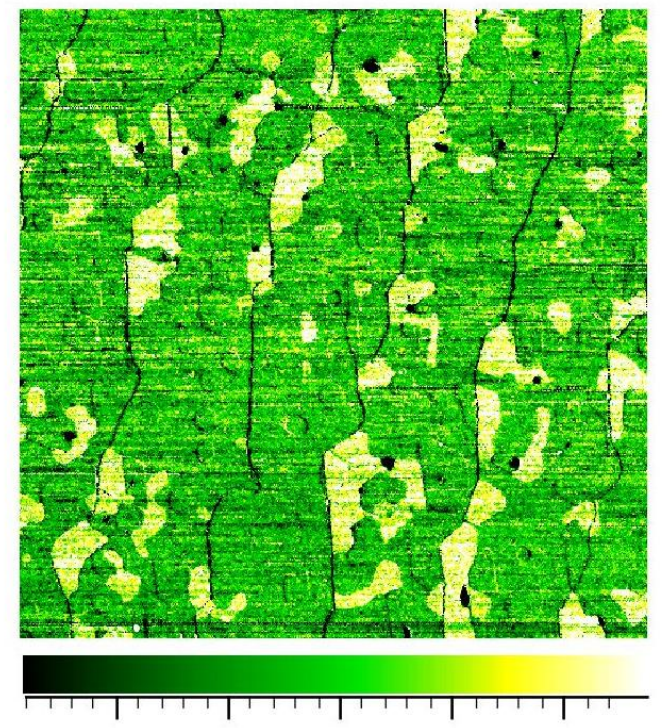
$\begin{array}{lllll}-0.50 & -0.25 & 0.00 & 0.25 & 0.50\end{array}$
Phase [Grad]

Abbildung 25: (a) $4 \times 4 \mu \mathrm{m}^{2}$ AFM-Topographie einer Graphen-SiC(0001) Probe. Die Breite der großen Terrassenstrukturen hat sich im Vergleich zum unpräparierten SiC-Substrat ungefähr verdreifacht, die Orientierung ist jedoch im Vergleich zum Substrat unverändert. Die mittlere Höhendifferenz der Terrassenstrukturen entspricht der von drei SiC-Bilagen. (b) Simultan aufgenommenes Phasenbild zeigt zwei Phasenkontraste, anhand derer ein- und zweilagiges Graphen identifiziert werden kann.

Dabei treten verschiedenste Stufenhöhen auf, die sich zum einen auf Substratstufen und zum anderen auf eine unterschiedliche Bedeckung dieser mit ein oder zwei Lagen Graphen zurückführen lassen. Auf den größeren Terrassen findet man eine große Anzahl von hexagonalen Löchern (eng. Pits) mit einer Fläche von $1 \mu \mathrm{m}^{2}$ bis einigen $\mu \mathrm{m}^{2}$. Die Orientierung der Kanten ist in den $\langle 11 \overline{2} 0\rangle_{\text {SiC }}$-Richtungen. Die großskalige Orientierung der Stufenkanten verläuft senkrecht zur [11̄00]-Richtung und entspricht damit der Stufenkantenorientierung des unbehandelten $\mathrm{SiC}(0001)$ Wafers. Außerdem weisen diese im Mittel eine Stufenhöhe von $\sim 750$ pm auf. Dies entspricht drei SiC-Doppellagen und bestätigt das durch StepBunching beeinflusste Graphenwachstum. Die Verteilung der Bereiche von ein- und zweilagigem Graphen weisen ebenso auf die Korrektheit des Wachstumsmodells von Hupalo et al. hin. In Abbildung 25 (b) ist die gleichzeitig zur Topographie aufgenommene relative Phasenänderung zwischen der Anregungs- und aktuellen Frequenz der AFM-Spitze gezeigt. Wie in Kapitel 3.5 dargestellt wird, zeigen die mit dem Rastertunnelmikroskop bei 
gleichen Präparationsparametern untersuchten Proben eine Bedeckung von ein- und zweilagigem Graphen. Daher ist hier davon auszugehen, dass die relative Phasenverschiebung durch die unterschiedliche Bedeckung von ein- und zweilagigem Graphen zustande kommt. Die Bereiche mit positiven Phasenschub in Abbildung 25 (b) sind hauptsächlich auf den unteren Terrassen der SiC-Dreifachstufen zu finden und können daher anhand ihrer Lage, wie man es vom Wachstumsmodell erwartet, zweilagigem Graphen zugeordnet werden. Eine alleinige Zuordnung mit Hilfe des rel. Phasenkontrastes ist jedoch nicht eindeutig möglich, da Stärke und Vorzeichen von den mechanischen und elektrischen Eigenschaften des AFM-Kontaktes abhängen. Daher wurde zusätzlich die räumliche Variation der Kontaktpotentialdifferenz zwischen Spitze und Probe bzw. das Oberflächenpotential der präparierten Oberflächen untersucht.

Abbildung 26 zeigt einen $2 \times 2 \mu \mathrm{m}$ großen Ausschnitt einer Graphenprobe, bei der neben der Topographie simultan das Oberflächenpotential (b) und die Phase (c) aufgenommen worden sind. Ebenso wie in der Phase beobachtet man mit der Topographie korrelierte Bereiche unterschiedlichen Oberflächenpotentials. Aufgrund der räumlichen Verteilung der Bereiche ist wiederum davon auszugehen, dass es sich bei den Bereichen höherer Kontaktpotentialdifferenz und damit niedrigerer Austrittsarbeit um zweilagiges und Bereiche mit niedrigerer Kontaktpotentialdifferenz und damit höherer Austrittsarbeit um einlagiges Graphen handelt. Daher besitzt an Luft einlagiges Graphen eine größere Austrittsarbeit als zweilagiges Graphen. Die Kontaktpotentialdifferenz zwischen ein- und zweilagigem Graphen beträgt $\Delta U_{\mathrm{CPD}, \mathrm{BL}-\mathrm{ML}}=144(10) \mathrm{mV}$ und ist mit bisherigen Messungen an Luft vergleichbar.

Untersuchungen der Austrittsarbeiten von Graphen im UHV sowohl mit dem AFM als auch der Photoemissionsspektroskopie zeigen jedoch einen umgekehrten Trend [53, 73], wie es auch von der Theorie erwartet wird. Die Austrittsarbeit nimmt demnach von einlagigem zu mehrlagigem Graphen zu. Ursache für die Diskrepanz zwischen UHV-Messungen und Messungen an Luft ist die Kontamination der Oberfläche. So gehen Brunett et al. davon aus, dass auf einlagigem Graphen an Atmosphäre im Gegensatz zu zweilagigem Graphen ein adsorbierter Wasserfilm liegt [74]. 
a)

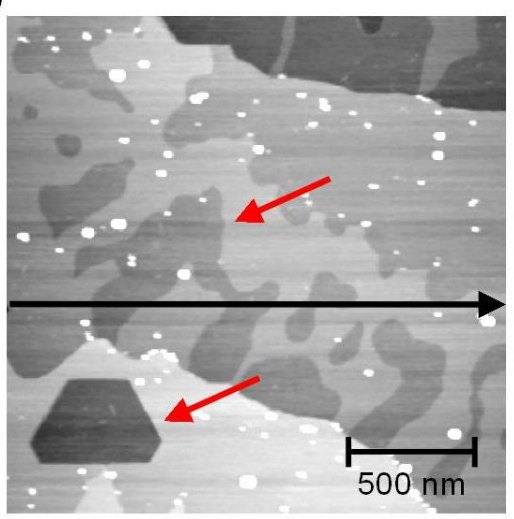

c)

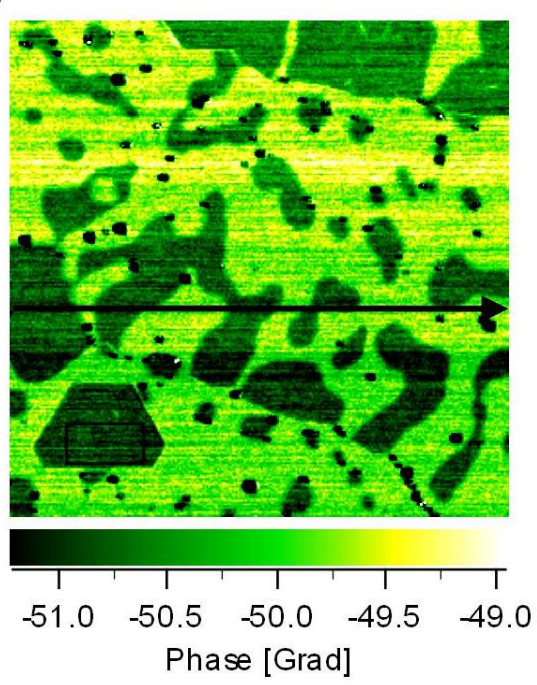

b)
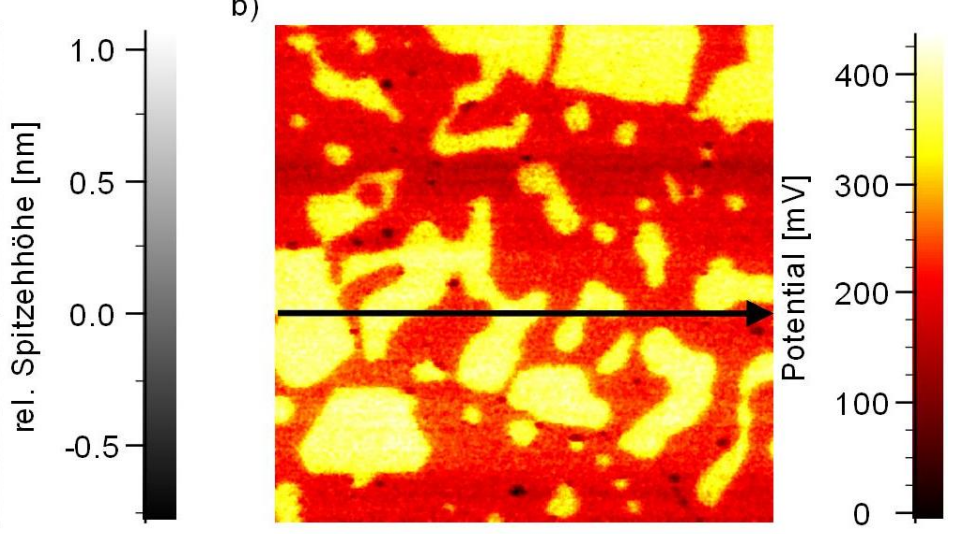

d)

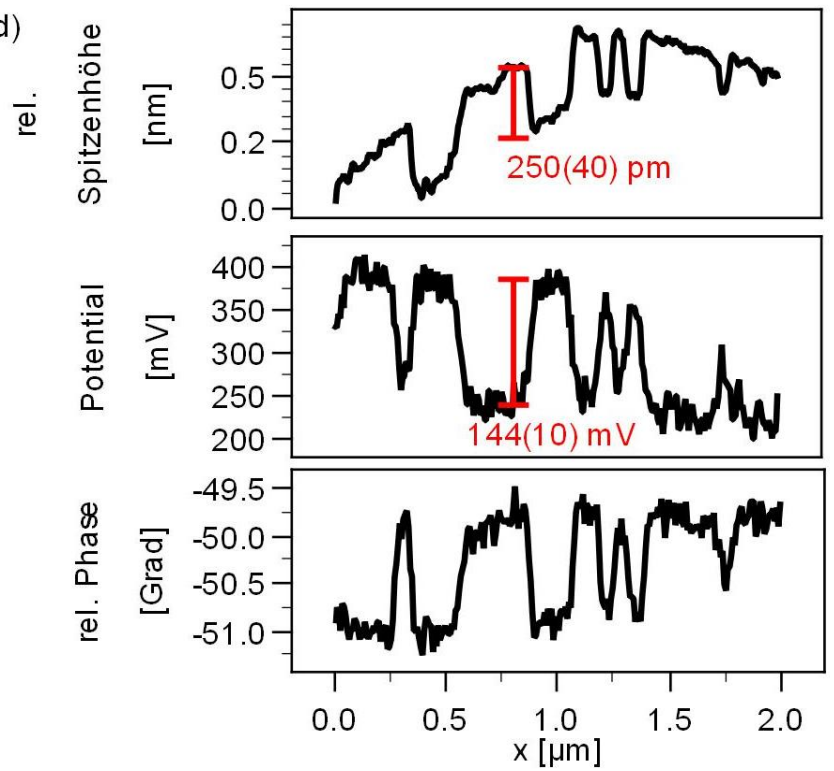

Abbildung 26: Bestimmung der lokalen Austrittsarbeit von ein- und zweilagigem Graphen an Luft. (a) $2 \times 2 \mu \mathrm{m}$ AFM-Topographie mit ein-und zweilagiger Graphen-Bedeckung. (b) Simultan aufgenommene Kontaktpotential- und (c) Phasendifferenz sowie (d) laterale Schnitte durch (a)-(c). Kontaktpotential- und Phasendifferenz sind räumlich korreliert und erlauben die Zuordnung der Graphenschichten. Die Kontaktpotentialdifferenz zwischen ein- und zweilagigem Graphen beträgt $\Delta U_{\mathrm{CPD}, \mathrm{ML}, \mathrm{BL}} \approx 144 \mathrm{mV}$.

Zusätzlich wurde der Einfluss des Umgebungsgases auf die Austrittsarbeiten von ein- und zweilagigem Graphen von Pearce et al. untersucht. Die Autoren zeigen, dass Umgebungsgase wie $\mathrm{O}_{2}, \mathrm{H}_{2} \mathrm{O}$ und $\mathrm{NO}_{2}$ zu einer höheren Austrittsarbeit auf einlagigem im Vergleich zu zweilagigem Graphen führen [75]. Die unterschiedliche Kontamination von ein- und zweilagigem Graphen mit u.a. einem Wasserfilm führt ebenso dazu, dass die erwarteten theoretischen Stufenhöhen zwischen ein- und zweilagigem Graphen mit AFM-Messungen an Atmosphäre nicht bestimmt werden können [74, 76]. So entspricht die Höhendifferenz zwischen der im Vergleich zu einlagigem Graphen niedrigeren zweilagigen Graphenschicht 
in Abbildung $26-250(40) \mathrm{pm}$ und $-670 \mathrm{pm}$, und entspricht damit keiner theoretisch erwarteten oder im UHV gemessenen Stufenhöhe. Daher ist eine Identifikation der Graphenlagen anhand von reinen Rasterkraft-Topographiemessungen an Atmosphäre ungeeignet. Vielmehr muss sowohl die Phaseninformation zusammen mit der gesamten Morphologie als auch das Oberflächenpotential bei der Bestimmung mittels AFM mit berücksichtigt werden. Die in dieser Arbeit untersuchten, und durch kurze Heizzyklen von etwa 2 min zwischen $1450{ }^{\circ} \mathrm{C}$ und $1600{ }^{\circ} \mathrm{C}$ präparierten Proben haben typische Graphenbedeckungen von $20 \%$ bis $60 \%$ mit zweilagigem Graphen. Die restliche Oberfläche ist mit einlagigem Graphen bedeckt. Größere Bereiche, welche mit unrekonstruierten SiC oder der Zwischenschicht-Rekonstruktion bedeckt sind, treten hauptsächlich bei niedrigeren Präparationstemperaturen auf. 


\subsection{Topographische und elektronische Untersuchung von ein- und zweilagigem Graphen mit dem Rastertunnelmikroskop}

\subsubsection{Einlagiges Graphen}

Abbildung 27 zeigt eine charakteristische atomar aufgelöste 14,5 $\times 14,5 \mathrm{~nm}^{2}$ Topographie von einlagigem Graphen. Im 1,2×1,2 $\mathrm{nm}^{2}$ Inset von Abbildung 27 (a) ist die Honigwabenstruktur des Graphengitters zu sehen. Im Gegensatz zu den LEED-Aufnahmen zeigt die Topographie zusätzlich zu dem Graphengitter eine quasi-periodische 6×6-Einheitszelle (vgl. Kapitel 3.4), die durch die Zwischenschicht hervorgerufen wird.

a)

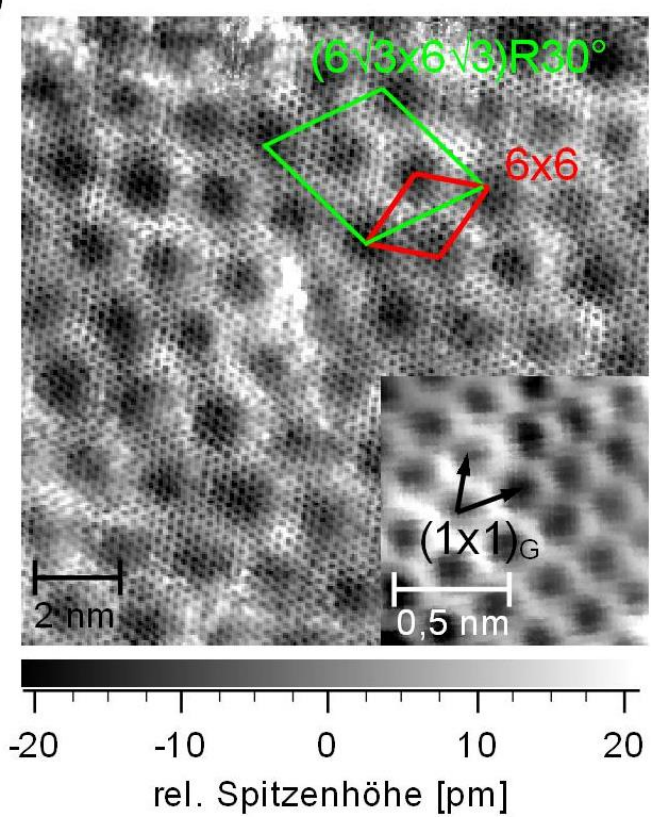

b)

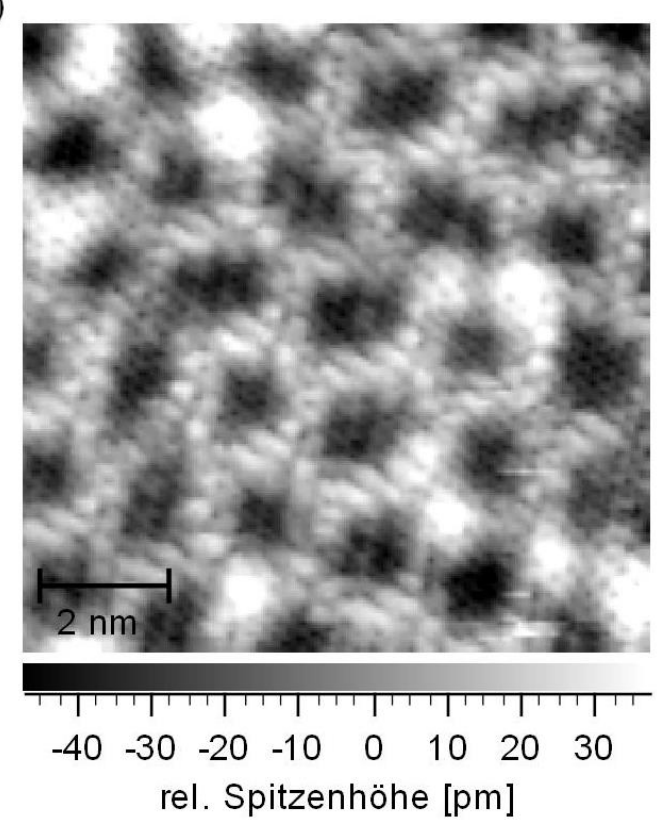

Abbildung 27: (a) $14,5 \times 14.5 \mathrm{~nm}^{2}$ große Topographie von einlagigem Graphen $\left(U_{\text {Bias }}=-0,04 \mathrm{~V}, I_{\mathrm{T}}=0,07 \mathrm{nA}\right)$. Die Topographie wird von zwei Korrugationen dominiert, dem Graphengitter mit der für einlagiges Graphen typischen Honigwabenstruktur (siehe 1,2×1,2 $\mathrm{nm}^{2}$ Inset) und der quasiperiodischen 6×6-Überstruktur, welche Höhenänderungen in der Größenordnung von $\sim 20$ pm in der Topographie hervorruft. Die jeweiligen Einheitszellen sind schwarz bzw. rot markiert. Zusätzlich ist in grün die Einheitszelle der $(6 \sqrt{3} \times 6 \sqrt{3})$ R30 $0^{\circ}$-Rekonstruktion eingezeichnet, welche sich jedoch in den STM-Daten zeigt. (b) $10 \times 10 \mathrm{~nm}^{2}$ großer Ausschnitt einer einlagigen Graphenschicht, aufgenommen bei höherer Spannung ( $\left.U_{\text {Bias }}=-0,5 \mathrm{~V}, I_{\mathrm{T}}=90 \mathrm{pA}\right)$. Die Graphen-Lage wird für höhere Tunnelspannungen zunehmend transparent, und es wird hauptsächlich die darunterliegende quasiperiodische Zwischenschicht abgebildet. 
Eine $5 \times 5$ Periodizität, wie von $[62,77,78]$, konnte hier bei keiner der verwendeten Proben beobachtet werden. Für höhere Tunnelspannungen (vgl. Abbildung 27 (b), $U_{\text {Bias }}=-0,5 \mathrm{~V}$ ) wird die obere Graphenlage transparent, und es werden zunehmend Zustände der rekonstruierten Zwischenschicht sichtbar [60].

Abbildung 28 zeigt Multi-Bias-Topographien im Energiebereich von -0,2 bis 0,2 V. Diese geben einen Einblick in die integrale lokale Zustandsdichte von einlagigem Graphen im gewählten Energieintervall $E_{\mathrm{F}} \pm U_{\text {Bias }}$. In den gewählten Energiebereichen treten räumlich lokalisierte Zustände als Erhöhungen in den Topographien auf, deren Ausdehnung einige Gitterkonstanten des Graphens betragen. Dabei treten sie an verschiedenen Positionen und bei unterschiedlichen Energien auf, wie z.B. der Vergleich der einzelnen Strukturen bei 0,04 und 0,04 V zeigt. Es gibt Kontraste, welche nur für besetzte Zustände, für unbesetzte sowie für beide auftreten (rot, grün und schwarz markiert).

a)
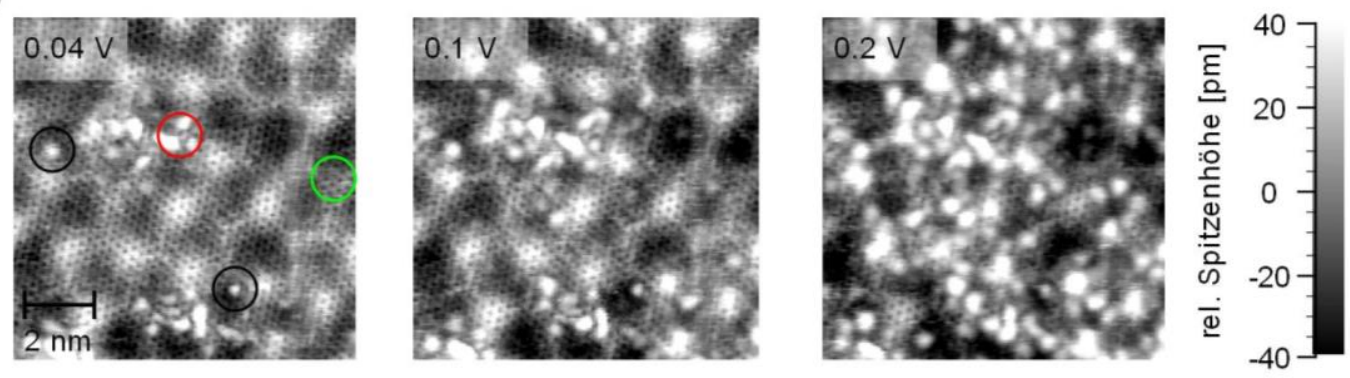

b)
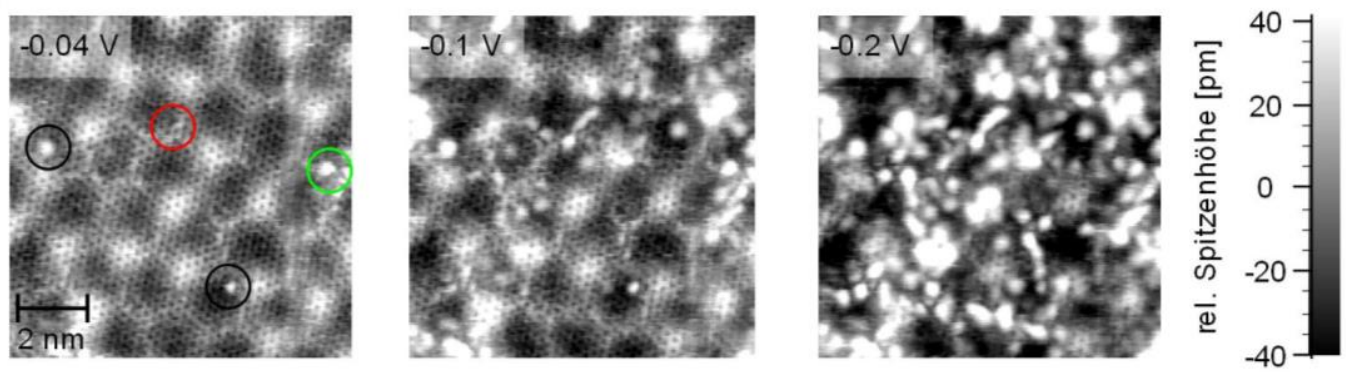

Abbildung 28: Multi-Bias-Topographien $\left(10 \times 10 \mathrm{~nm}^{2}, I_{\mathrm{T}}=0,15 \mathrm{nA}\right)$ von einlagigem Graphen für (a) positive Spannungen von 0,04 V, 0,1 V und 0,2 V und (b) negative Spannungen von -0,04 V, -0,1 V und -0,2 V. Für Energien nahe der Fermi-Energie (-0,04 bis 0,04 eV) sind neben der Honigwabenstruktur des Graphengitters sowie der 6×6 Überstruktur vereinzelt in Abhängigkeit der Bias-Spannung unterschiedliche räumlich lokalisierte Erhöhungen erkennbar (schwarze, rot, grün). Diese werden im Allg. lokalisierten Defekt-Zuständen in der Zwischenschicht zugeschrieben. Die Defekt-Zustände erstrecken sich dabei über mehrere $100 \mathrm{meV}$ um die Fermi-Energie. 
Vergleichbare Kontraste in den Topographien wurden ebenso im Energieintervall von -0,2 bis $-0,5 \mathrm{~V}$ und 0,2 bis $0,5 \mathrm{~V}$ beobachtet $[60,79,80]$.

Der elektronische Einfluss der Zwischenschicht auf die Graphenlage, selbst bei der FermiEnergie, wird durch Messungen der differentiellen Leitfähigkeit auf einlagigem Graphen deutlich. Abbildung 29 (a) zeigt eine $7 \times 7 \mathrm{~nm}^{2}$ große Topographie, zu welcher an jedem Messpunkt eine I(U)-Kennlinie im Spannungsbereich von -168 mV bis $162 \mathrm{mV}$ aufgenommen worden ist. Das über den gesamten Bereich gemittelte dI/dU-Signal ist in Abbildung 29 (b) zusammen mit Einzelspektren an vier verschiedenen Orten mit unterschiedlichen Signaturen dargestellt. Die Einzelspektren weichen deutlich vom gemittelten Spektrum ab und weisen lokalisierte Zustände in der differentiellen Leitfähigkeit bei -92 mV, -53 mV, $34 \mathrm{mV}$ und $35 \mathrm{mV}$ auf. Die Leitfähigkeitskarten in Abbildung 29 (c) zeigen die räumliche Verteilung und die Ausdehnung der entsprechenden Zustände. Auch hier zeichnet sich keine räumlich geordnete Struktur der gefundenen Signaturen ab. Außerdem sind die lokalisierten Zustände über ein breites energetisches Spektrum verteilt. Die Ausdehnung dieser Zustände liegt bei unter 0,5 nm bis hin zu einigen $\mathrm{nm}$. Ebenso zeigt sich, dass diese Defekte die elektronische Struktur des einlagigen Graphens um die Fermi-Energie deutlich beeinflussen (vgl. Abbildung 29 (c), dI/dU-Karte bei 0 V). Die erste Graphenlage ist demnach selbst bei der Fermi-Energie nicht vollständig von der Zwischenschicht entkoppelt und wird stark von dessen Defekten beeinflusst. Messungen der differentiellen Leitfähigkeit von Cervenka et. al. zeigen eine erhöhte differentielle Leitfähigkeit bei $\pm 500 \mathrm{mV}$ und $\pm 200 \mathrm{mV}$ an Orten der lokalisierten Zustände [79, 80]. Sie vermuten, dass es sich bei den lokalisierten Zuständen bei $500 \mathrm{mV}$ und $200 \mathrm{mV}$ hauptsächlich um abgesättigte SiliziumDangling-Bonds zwischen dem SiC-Substrat und der Zwischenschicht handeln muss. Dabei stützen sie Ihre Beobachtungen auf Photoemissionsdaten, welche bisher vermutlich auflösungsbedingt zwei lokalisierte Zustände der Zwischenschicht bei $-0,5 \mathrm{eV}$ und 1,6 eV identifiziert haben. Die hier in den eigenen STM-Daten zusätzlich gefundenen lokalisierten Zustände im Energiebereich zwischen $\pm 100 \mathrm{mV}$ sowie dessen aperiodisches Auftreten zeigt, dass es sich bei der Zwischenschicht nicht um eine perfekte, sondern um eine stark durch verschiedene Defekte geprägte Rekonstruktion handeln muss. Dabei kann es sich z.B. um Fehlstellen, gesättigte oder auch nicht gesättigte Silizium-Dangling-Bonds und eingeschlossene Adsorbaten, wie Wasserstoff handeln. 
a)

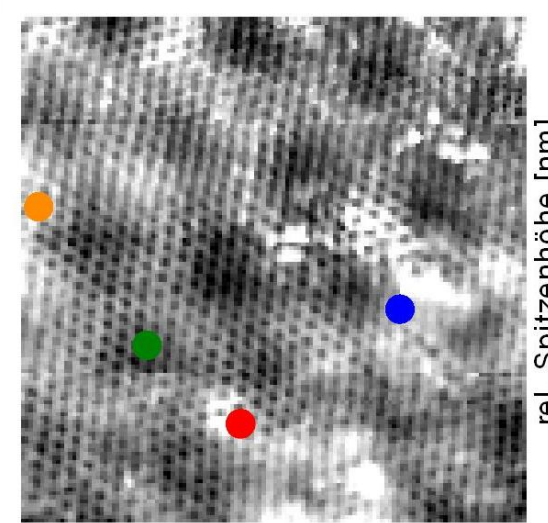

c)

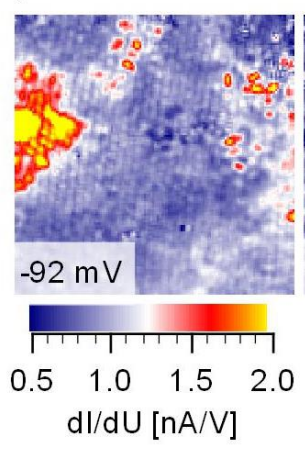

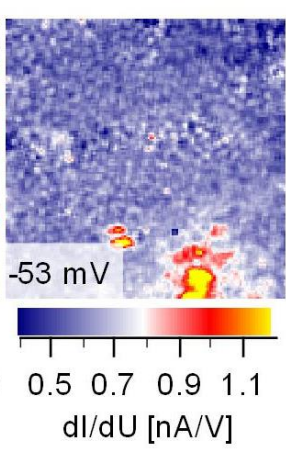

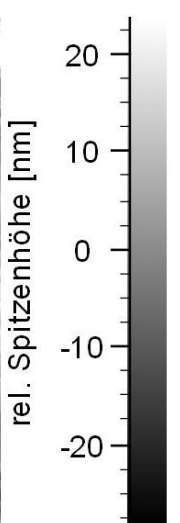

b)

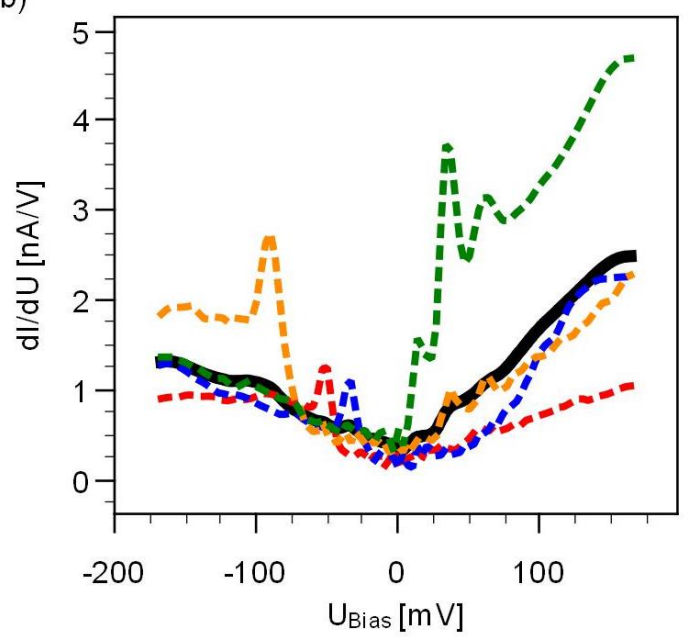

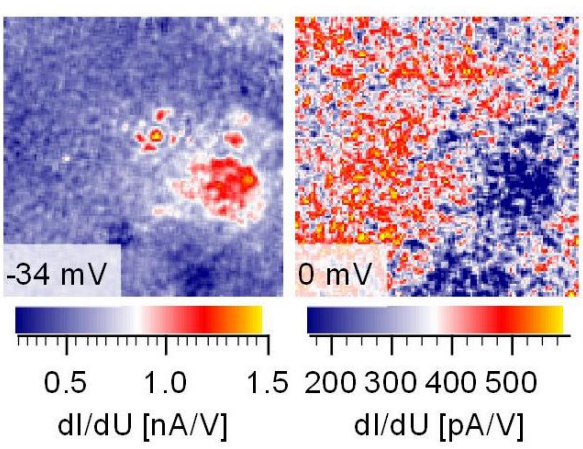

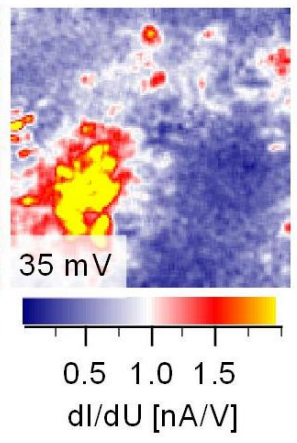

Abbildung 29: (a) $7 \times 7 \mathrm{~nm}^{2}$ große Topographie und (b) über gesamten Bildausschnitt gemitteltes dI/dU-Signal (schwarze Linie) sowie ausgewählte Einzelspektren an verschiedenen räumlichen Positionen (vgl. Topographie) mit ausgeprägten Peaks bei $-92 \mathrm{mV},-53 \mathrm{mV},-34 \mathrm{mV}, 35 \mathrm{mV}$ (orange, rote, grüne und blau Linie), welche auf lokalisierte Zustände des Interface-Layer hindeuten. (c) Räumliche dI/dU-Karten bei entsprechenden Energien zeigen, die unterschiedliche räumliche Verteilung der einzelnen Zustände und deren räumliche Ausdehnung von bis zu einigen nm. Selbst bei der Fermi-Energie ( $0 \mathrm{~V})$ sind räumliche Schwankungen im dI/dU-Signal sichtbar.

Aufgrund der starken räumlichen Variation des dI/dU-Signals ist die Angabe eines „Standardspektrums" für einlagiges Graphen anhand der eigenen Daten nicht möglich und erklärt möglicherweise auch die große Varianz in der bisher veröffentlichten Literatur [47, 79, 81, 82]. Nach dem Tight-Binding-Ansatz, welcher die mit Hilfe der Photoemission bestimmte Dispersionsrelation gut beschreibt [19], erwartet man für n-dotiertes einlagiges Graphen eine lineare Zustandsdichte nahe der Fermi-Energie. Alle bisher veröffentlichten Spektroskopien auf epitaktischem Graphen auf SiC(0001) weisen jedoch die Gemeinsamkeit auf, dass die differentielle Leitfähigkeit um die Fermi-Energie im Bereich von ca. $\pm 100 \mathrm{mV}$ auf einen endlichen Wert einbricht $[47,79,82]$ oder sich sogar auf Null reduziert [81]. Dieser 
experimentelle Befund wird auch als „Pseudogap“ bezeichnet, wobei seine Ursache bisher nicht abschließend geklärt werden konnte. Dabei wurde als mögliche Ursache zunächst ein phononenunterstützter Tunnelprozess, wie er auf einlagigem Graphen auf $\mathrm{SiO}_{2}$ gefunden worden ist [83], als mögliche Ursache diskutiert [81], inzwischen jedoch wiederum ausgeschlossen [79].

\subsubsection{Zweilagiges Graphen}

a)

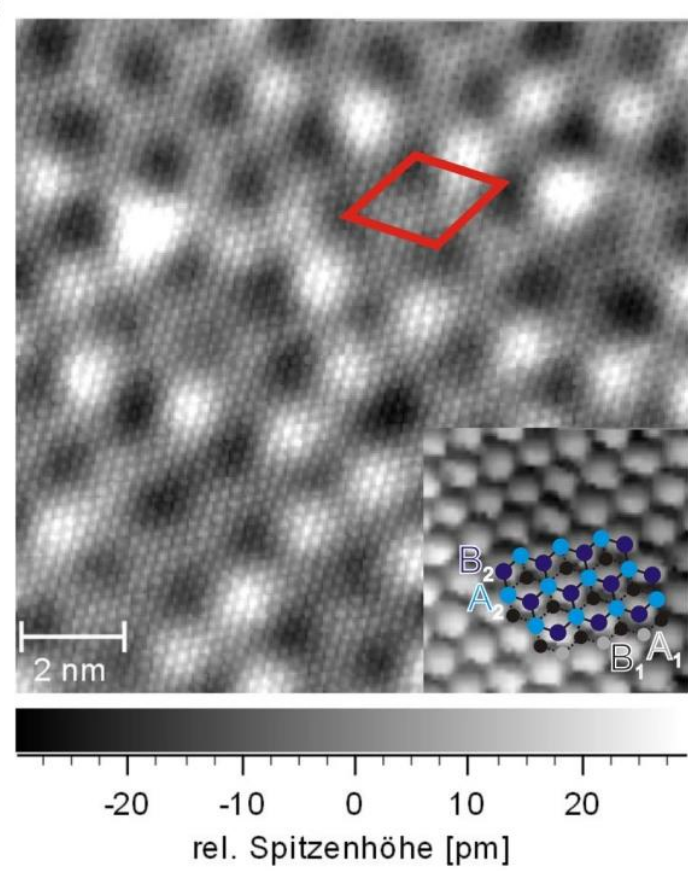

b)

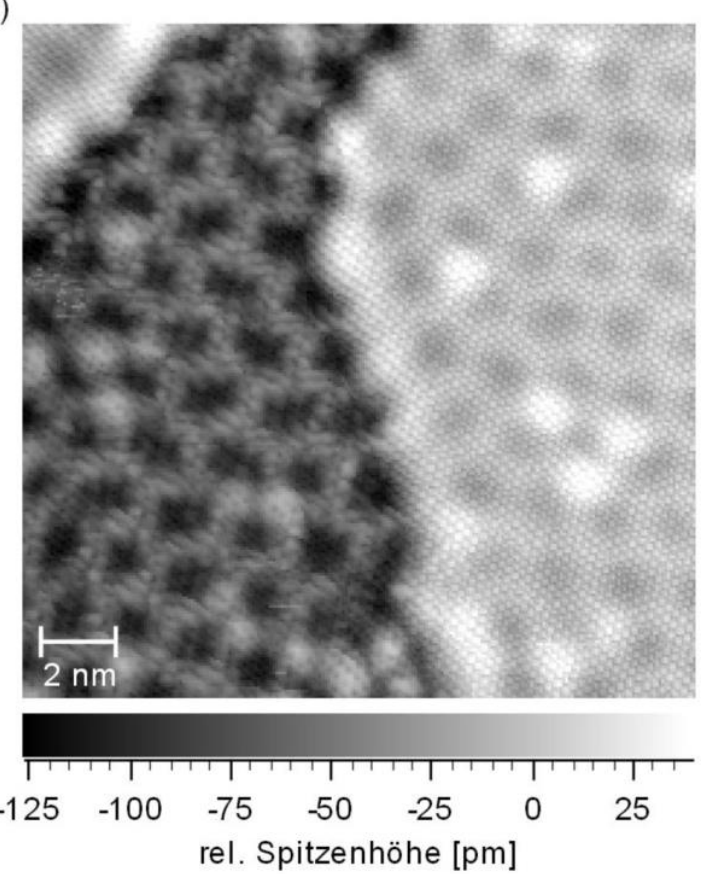

Abbildung 30: (a) $13 \times 13 \mathrm{~nm}^{2}$ großer Ausschnitt zweilagigen Graphens. Die Topographie zeigt wie bei einlagigem Graphen die hexagonale Struktur des Graphengitters sowie dem überlagert die 6×6-Periodizität der Zwischenschicht $\left(U_{\text {Bias }}=-0,56 \mathrm{~V}, I_{\mathrm{T}}=60 \mathrm{pA}\right)$. Abhängig von der Energie wird jedoch nur eines der beiden Graphen-Untergitter abgebildet. (b) Direkter Vergleich mit einlagigem Graphen an einem ML-BL-Übergang. Zweilagiges Graphen weist zwar ebenso die 6×6-Periodizität der Zwischenschicht auf, zeigt aber im Gegensatz zu einlagigem Graphen keine einzelnen Strukturen innerhalb einer Einheitszelle der Zwischenschicht. Außerdem ist die Modulationsamplitude der 6×6-Periodizität deutlich reduziert $\left(18 \times 18 \mathrm{~nm}^{2}, U_{\text {Bias }}=-0,5 \mathrm{~V}\right.$, $\left.I_{\mathrm{T}}=90 \mathrm{pA}\right)$.

Abbildung 30 (a) zeigt einen $13 \times 13 \mathrm{~nm}^{2}$ großen Ausschnitt von zweilagigem Graphen. Die Oberfläche zeigt ebenso wie die erste Graphenlage eine 6×6-Periodizität (Einheitszelle rot markiert), wobei die rel. Höhenänderungen geringer ausfallen. Ein direkter Vergleich bei- 
der Graphenlagen ist in Abbildung 30 (b) zu sehen. Die unter ein- und zweilagigem Graphen liegende Zwischenschicht ist durchgängig. Die Modulationsamplitude der 6×6-Periodizität ist reduziert, wie ebenso durch andere STM-Gruppen beobachtet worden ist [47, $60,81,84]$.

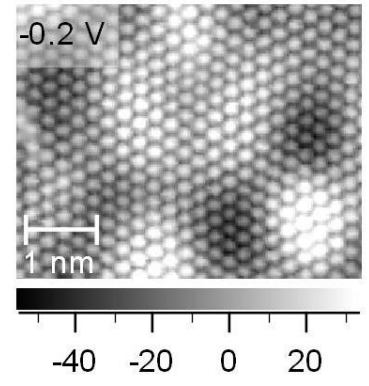

rel. Spitzenhöhe [pm]

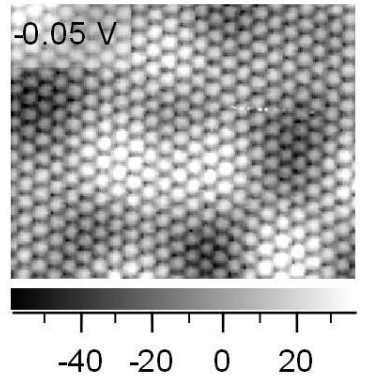

rel. Spitzenhöhe $[\mathrm{pm}]$

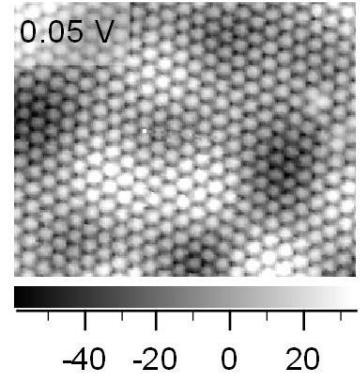

rel. Spitzenhöhe $[\mathrm{pm}]$

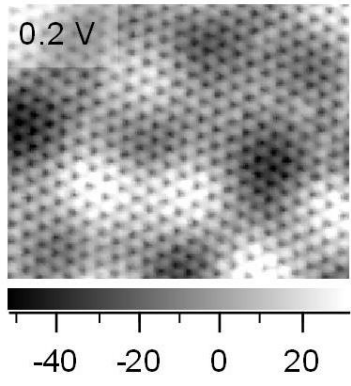

rel. Spitzenhöhe $[\mathrm{pm}]$

Abbildung 31: Topographien desselben Probenbereiches von zweilagigem Graphen, aufgenommen bei unterschiedlichen Spannungen (v. 1. n. r: -0,2 V, -0,05 V, 0,05 V und 0,2 V). Im Vergleich zu einlagigem Graphen wird nur eines der beiden Untergitter für Energien nahe $\mathrm{E}_{\mathrm{F}}$ abgebildet. Lokalisierte Zustände wie auf einlagigem Graphen sind nicht vorhanden.

Im Gegensatz zu einlagigem Graphen zeigt zweilagiges Graphen für niedrige Spannungen um $\mathrm{E}_{\mathrm{F}}$ eine trigonale Periodizität. Daher wird aufgrund von STM-Daten angenommen, dass zweilagiges Graphen auf SiC(0001) eine AB-Stapelfolge (engl. Bernal-Stacking) besitzt [47, 60, 85]. Da bei der AB-Stapelfolge nur unter einem der beiden Untergitter (o. B. d. A. $\mathrm{A}_{2}$ ) der oberen Lage ein Atom der unteren Lage sitzt (Untergitter $\mathrm{B}_{1}$ ), sind die beiden Untergitter $\left(\mathrm{A}_{2}, \mathrm{~B}_{2}\right)$ der oberen Lage nicht äquivalent. Dies führt im Energiebereich der Zwischenschichtkopplung um $\mathrm{E}_{\mathrm{D}}$ zu einer reduzierten Zustandsdichte für das Untergitter $\mathrm{A}_{2} \mathrm{im}$ Vergleich zum Untergitter $B_{2}$ [60]. Daher wird bei niedrigen Spannungen nahe der FermiEnergie nur eines der beiden Untergitter in den STM-Topographien abgebildet, wie in Abbildung 30 (a) und Abbildung 31 zu sehen ist. Die Korrugation im Spannungsbereich von $-0,2 \mathrm{~V} \leq U_{\mathrm{Bias}}<0,2 \mathrm{~V}$ ändert sich nicht wesentlich und zeigt das trigonale Gitter. Es tragen hauptsächlich Zustände des Leitungs- bzw. Valenzbandes, welche die Kopplung zwischen dem Untergitter $\mathrm{B}_{2}$ und $\mathrm{A}_{1}$ sowie der nächsten Nachbarn innerhalb einer Graphenlage berücksichtigen, zum Tunnelstrom bei. Mit Einsetzen des zweiten Leitungsbandes ändert sich die Korrugation und ein Teil der Elektronen tunnelt in die unbesetzten Zustände des zweiten Leitungsbandes, wie in Abbildung 31 für $U_{\text {Bias }}=0,2 \mathrm{~V}$ sichtbar ist. Für höhere Spannungen wird wiederum die hexagonale Struktur des Graphengitters beobachtet [85]. 
Stark lokalisierte Zustände der Zwischenschicht, wie sie in den Topographien von einlagigem Graphen auftreten, sind nicht zu erkennen, wie ein Vergleich der Abbildung 31 und Abbildung 28 zeigen. Der verminderte Einfluss der lokalisierten Zustände auf die zweite Graphenlage kann mit dem größeren vertikalen Abstand der zweiten Graphenlage von der Zwischenschicht begründet werden.

Um die elektronischen Eigenschaften von zweilagigem Graphen zu charakterisieren, wurden ebenso wie auf einlagigem Graphen Messungen der differentiellen Leitfähigkeit durchgeführt. Dazu wurden an $128 \times 61$ topographischen Messpunkten (vgl. Abbildung 32 (a)) einzelne I(U)-Kurven aufgenommen und anschließend nummerisch differenziert. Beispielhaft sind drei Einzelspektren im Inset von Abbildung 32 (b) dargestellt. Die über den gesamten Probenbereich gemittelte differentielle Leitfähigkeit ist in Abbildung 32 (b) gezeigt. Die über den Probenbereich gemittelte differentielle Leitfähigkeit stimmt qualitativ mit bisherigen Messungen der differentiellen Leitfähigkeit auf epitaktischem Graphen überein $[47,85,86]$. Die Spektren weisen einen Einbruch der differentiellen Leitfähigkeit um 0,32 eV mit einer Breite von ca. $110 \mathrm{meV}$ sowie einen Anstieg der Leitfähigkeit bei ca. $150 \mathrm{meV}$ auf. Diese Merkmale der Spektren werden der energetischen Position des DiracPunkt $E_{\mathrm{D}}$, der Bandlücke $\mathrm{E}_{\mathrm{G}}$ und dem Einsetzen des zweiten Leitungsbandes $E_{\mathrm{L}, 2}$ zugeordnet. Berechnungen der Zustandsdichte von Lauffer et al. [47] anhand des in Kapitel 1.4 beschriebenen Tight-Binding-Modells von zweilagigem Graphen zeigen für die genannten Merkmale eine gute Übereinstimmung zwischen der berechneten Zustandsdichte aus Abbildung 32 (c) und der experimentellen differentiellen Leitfähigkeit aus Abbildung 32 (b). Die aus den Spektren extrahierten Werte für $E_{\mathrm{D}}, E_{\mathrm{G}}$ und $E_{\mathrm{L}, 2}$ stimmen ebenso mit ermittelten Werten aus Photoemissionsspektroskopie-Experimenten überein [19]. 
a)

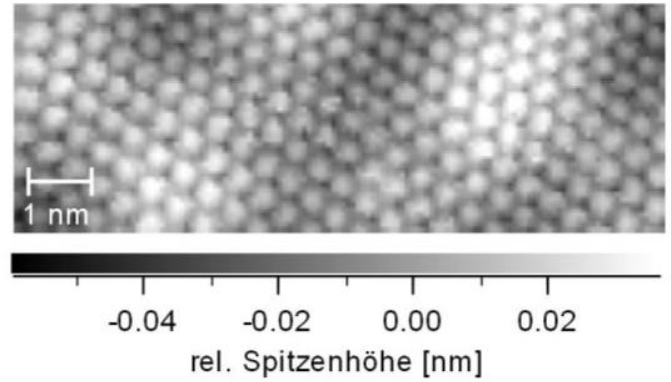

b)

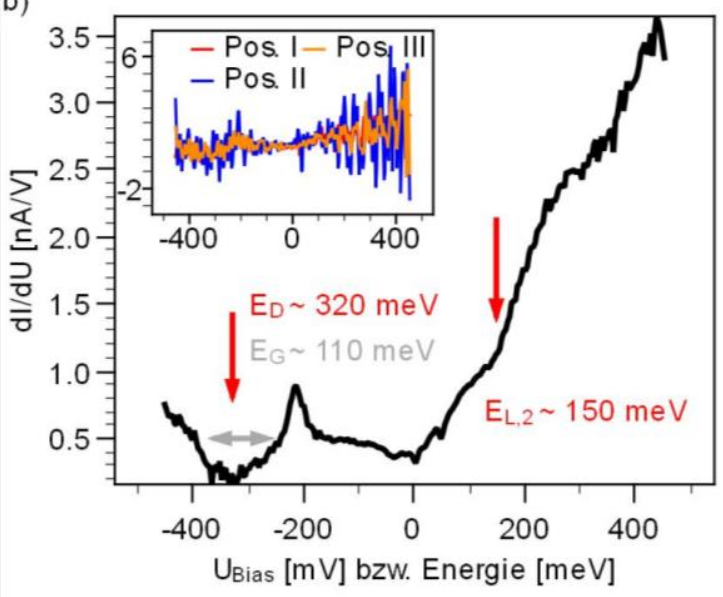

c)

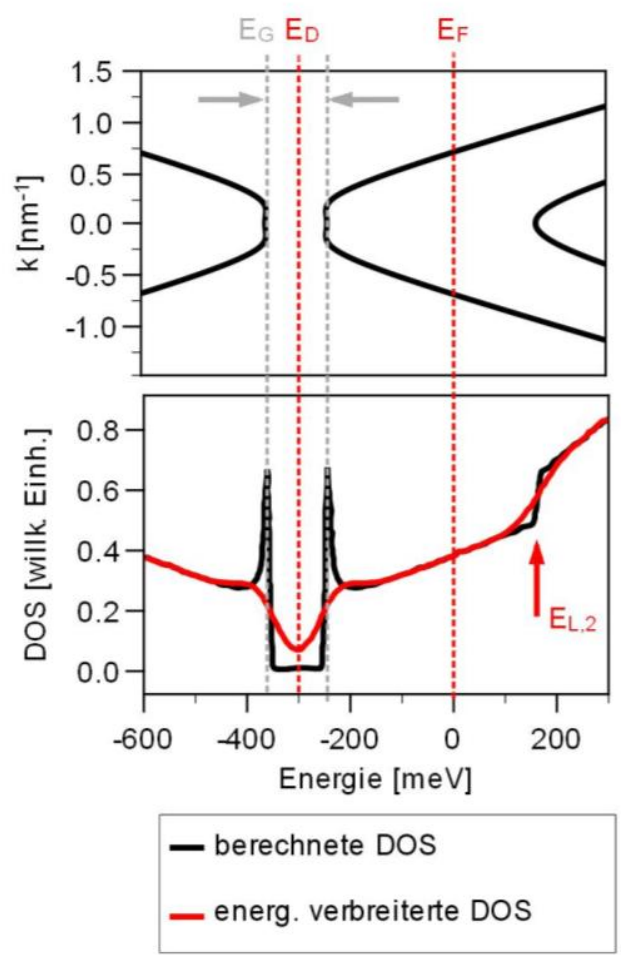

Abbildung 32: (a) Topographie $\left(5,2 \times 1,8 \mathrm{~nm}^{2}, U_{\text {Bias }}=-0,455 \mathrm{~V}, I_{\mathrm{T}}=200 \mathrm{pA}\right)$ von zweilagigem Graphen und (b) aus dem Bildbereich gemitteltes dI/dU-Signal von 7808 Einzelspektren. Die räumliche Mittelung über die Einzelspektren ist aufgrund des schlechten Signal-zu-Rausch-Verhältnisses nötig. Zum Vergleich sind Einzelspektren im Inset von (b) zu sehen. Anhand der charakteristischen spektroskopischen Merkmale können der Dirac-Punkt $E_{\mathrm{D}}$, die Bandlücke $E_{\mathrm{G}}$ und das Einsetzten des zweiten Leitungsbandes $E_{\mathrm{L}, 2}$ bestimmt werden (durch Pfeile markiert). (c) Tight-Binding-Bandstruktur und berechnete Zustandsdichte nach exp. Daten von Lauffer u.a. ohne und mit energetischer Verbreiterung der Zustandsdichte von 0,2 eV [47]. Der direkte Vergleich liefert eine gute Übereinstimmung. 


\subsubsection{Weitere Defekte - Eigenschaften von ein- und zweilagigem Graphen sowie der Zwischenschicht}

a)

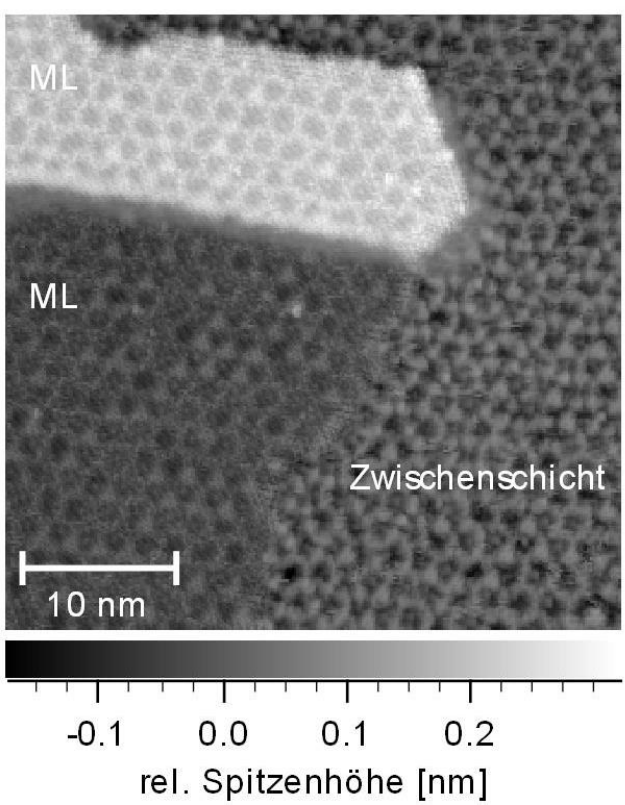

b)

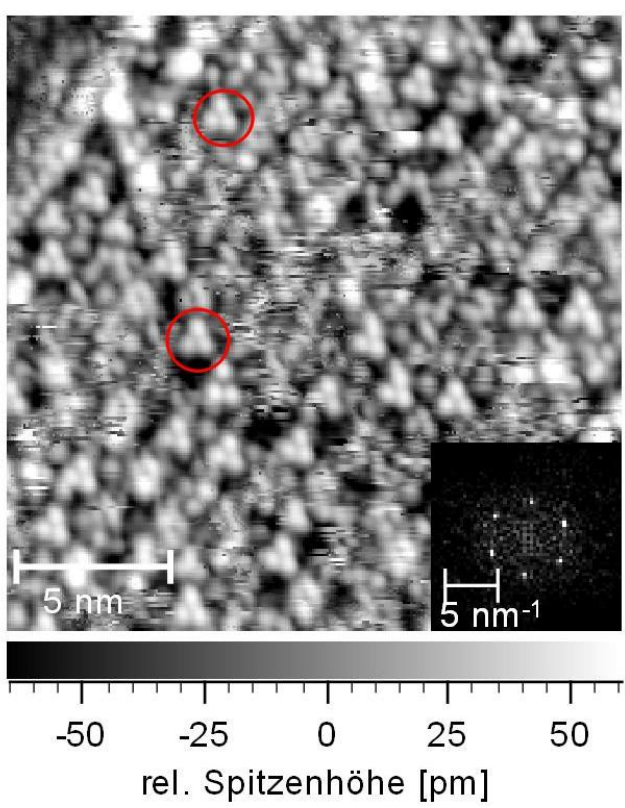

Abbildung 33: (a) STM-Topographie $\left(U_{\text {Bias }}=-1,4 \mathrm{~V}, I_{\mathrm{T}}=80 \mathrm{pA}, 40 \times 40 \mathrm{~nm}^{2}\right)$ mit einlagigem Graphen (ML) auf zwei Terrassen umgeben von der Zwischenschicht. (b) Vergrößerter Bereich der Zwischenschicht-Lage $\left(U_{\text {Bias }}=-1,4 \mathrm{~V}, I_{\mathrm{T}}=100 \mathrm{pA}, 20 \times 20 \mathrm{~nm}^{2}\right)$. Der Ausschnitt der FFT zeigt die Spots der quasiperiodischen $6 \times 6$ Einheitszelle mit der dazugehörigen Periodizität von $\sim 18 \AA$. Es sind zahlreiche Trimere auf der ansonsten unstrukturierten Oberfläche zu erkennen.

Abbildung 33 zeigt einen ML-ML-Übergang mit einer darunterliegenden Substratstufe sowie einen angrenzenden Bereich, der keine Graphensignaturen besitzt. Eine vergrößerte Aufnahme in Abbildung 33 (b) zeigt, dass es sich dabei um die Zwischenschicht handelt, die nicht durch darüberliegendes epitaktisches Graphen bedeckt ist. Die FFT-Darstellung im Inset von Abbildung 33 (b) zeigt hexagonal angeordnete Spots, welche der Periodizität des hexagonalen Gitters von $6 \cdot \mathrm{a}_{\mathrm{SiC}}=18,42 \AA$ entsprechen. Ein Graphengitter ist nicht erkennbar, wie ebenso von S. W. Poon berichtet wird [64]. Durch den direkten Vergleich mit der angrenzenden Graphenlage ist eine eindeutige Identifizierung der Zwischenschicht möglich.

Abbildung 34 (a) zeigt die Eigenschaften der Zwischenschicht bei der Bedeckung mit einbzw. zweilagigem Graphen. Die Zwischenschicht scheint eine starke Unordnung aufzuweisen, wie die Topographie aus Abbildung 34 (a) zeigt. Im linken Teil der Topographie ist die 
Oberfläche mit einlagigem und im rechten Teil mit zweilagigem Graphen bedeckt. Auf die elektronischen Eigenschaften von zweilagigem Graphen wurde in 3.5.2 eingegangen. Die aufgrund der Zwischenschicht auftretende 6×6-Periodizität ist im Vergleich zum unbedeckten Fall besser erkennbar. Die Periodizität wird durch eine Versetzung gestört, welche offensichtlich die Zwischenschicht entlang mehrerer Einheitszellen verzerrt, wie im linken oberen Abschnitt des Bildes durch weiße Striche markiert ist. Außerdem sind zahlreiche Erhöhungen sowohl auf dem einlagigem als auch zweilagigem Graphen sichtbar. Diese haben auf einlagigem Graphen wie im unbedeckten Fall die Struktur von Trimeren oder auch Dimeren $\left(\left|U_{\text {Bias }}\right| \geq 0,5 \mathrm{~V}\right)$, und wurden unter anderem ebenso von Rutter et. al. beobachtet [60]. Auf zweilagigem Graphen erscheinen diese Defekte als gewölbte Erhöhungen. Die atomare Korrugation des Graphengitters bleibt dabei erhalten, wie in Abbildung $27 \mathrm{zu}$ sehen ist.

Die Dichte der Defekte ist annähernd auf beiden Lagen identisch und beträgt $\mathrm{n}_{\mathrm{D} \approx} 0,05 / \mathrm{nm}^{2}$. Daher ist anzunehmen, dass es sich bei den Defekten scheinbar um den selben Defekttyp der Zwischenschicht handelt. Die genaue Art des Defektes wird noch kontrovers diskutiert. So handelt es sich nach dem in Kapitel 3.3 vorgestellten Adatom-Modell von Hu et. al. um Si-Adatome einer siliziumreichen Zwischenschicht, deren Zwischenräume mit amorphen Kohlenstoffringen gefüllt ist [65]. Nach dem Modell der $6 \sqrt{ } 3 \times 6 \sqrt{3}$-rekonstruierten Zwischenschicht muss es sich um offene Si-Bindungen des Substrates handeln, die aufgrund der Gitterfehlanpassung keine Bindungen zu den ansonsten kovalent gebundenen Kohlenstoffatomen der Zwischenschicht zu den darunterliegenden Si-Atomen besitzen [87]. In beiden Modellen handelt es sich um offene Si-Bindungen. Nimmt man das Modell von $\mathrm{Hu}$ et. al. an, entspricht jedes Trimer drei Siliziumatomen mit jeweils einer ungesättigten Bindung. Es ist naheliegend anzunehmen, dass diese als Donatoren für die darüberliegende Graphenschicht fungieren und jedes Trimer drei Elektronen an die Graphenschicht abgibt. Mit der gegebenen Defektdichte erhält man eine defektinduzierte Elektronenkonzentration des epitaktischen Graphens von $\mathrm{n}=1,5 \cdot 10^{13} \mathrm{~cm}^{-2}$, welche gut mit der experimentell in Kapitel 4.2 über die Fermiwellenlänge bestimmten Elektronenkonzentration der Graphenschicht von $\mathrm{n}=1,3(2) \cdot 10^{13} \mathrm{~cm}^{-2}$ übereinstimmt.

Eine in dieser Arbeit beobachtete Eigenschaft dieser Defekte sowohl auf ein- als auch zweilagigem Graphen ist, dass einige dieser nicht positionsfest sind, wie zwei hintereinander 
aufgenommene Topographien am selben Ort in Abbildung 34 (b) und (c) zeigen. Die Dauer einer Aufnahme betrug jeweils 13 min. Die Positionen, bei denen Defekte von Abbildung 34 (a) zu Abbildung 34 (b) nicht mehr sichtbar sind, sind rot markiert, Defekte, die sich neu bilden, sind grün markiert. Die Ursache für diese Oberflächenmodifikationen kann anhand der Datenlage nicht geklärt werden. Es kann sich z.B. unabhängig von der Anwesenheit der STM-Spitze um thermisch induzierte Oberflächendiffusion der entsprechenden Adatome handeln, oder aber es kann durch Wechselwirkung der Spitze mit der Oberfläche zu den Modifikationen kommen.

a)

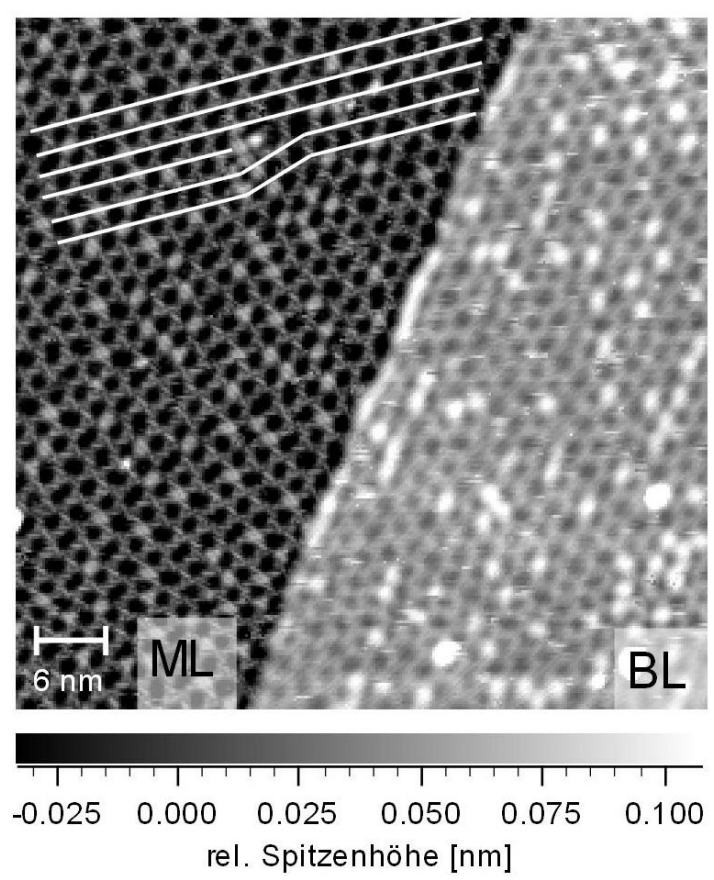

b)

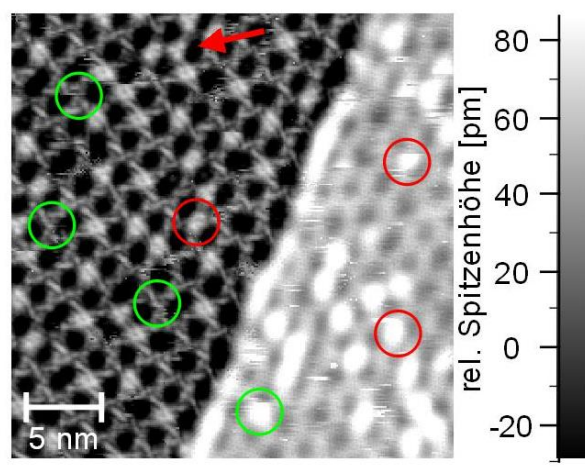

c)

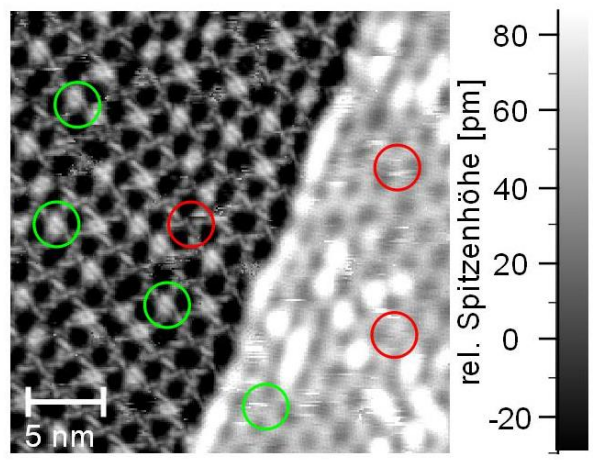

Abbildung 34: (a) 60×60 $\mathrm{nm}^{2}$ Topographie einer ein- und zweilagigen Graphen-Region (ML links, BL rechts, $\left.I_{\mathrm{T}}=50 \mathrm{pA}, U_{\mathrm{Bias}}=-2 \mathrm{~V}\right)$. Die unter der Monolage liegende Zwischenschicht zeigt zwar eine langreichweitige 6×6-Periodizität, die Einheitszellen weisen jedoch Irregularitäten auf. Im oberen Bildrand ist eine Versetzung des Interfaces durch weiße Linien markiert. Es sind zahlreiche Interface-Defekte sowohl auf ein- als auch zweilagigem Graphen erkennbar, wie in (b) durch einen roten Pfeil markiert ist. (b) und (c) Die Oberflächendefekte sind nicht statisch, wie die zwei aufeinanderfolgenden Bilder zeigen. Rote Kreise markieren Defekte, welche in Bild (b) zu sehen sind und in Bild (c) nicht zu sehen sind, grüne Kreise entsprechend umgekehrt $\left(30 \times 30 \mathrm{~nm}^{2}, I_{\mathrm{T}}=50 \mathrm{pA}, U_{\text {Bias }}=-2 \mathrm{~V}\right)$.

Neben den hier beschriebenen Punktdefekten der Zwischenschicht treten zwei weitere Defekte auf ein- und zweilagigem Graphen auf. Abbildung 35 zeigt eine $600 \times 600 \mathrm{~nm}^{2}$ große 
Übersichts-Topographie mit einer Bedeckung von ein- und zweilagigem Graphen mit unterschiedlicher Stufenhöhen.

a)

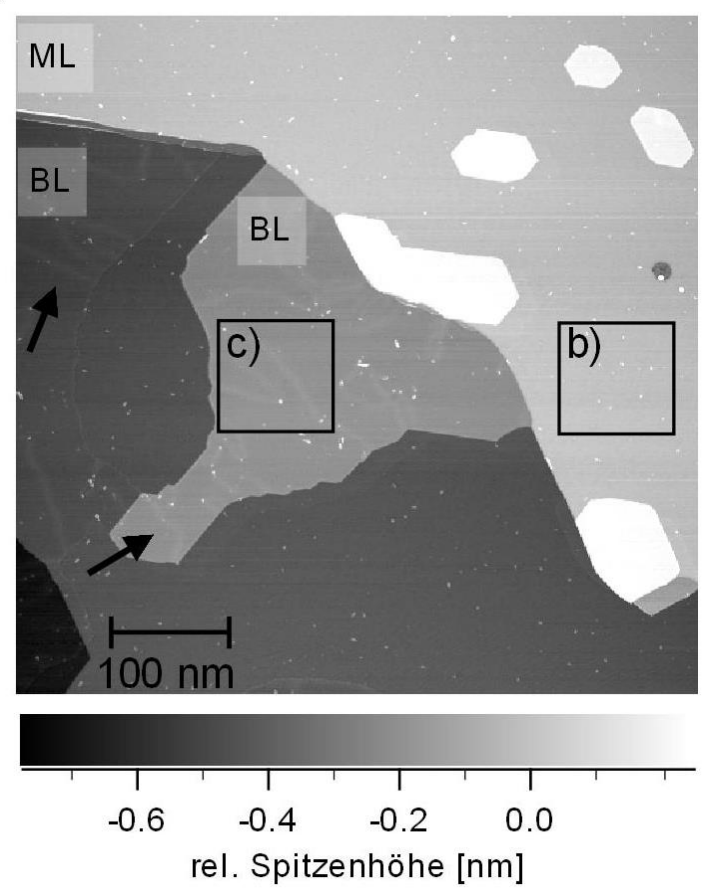

b)
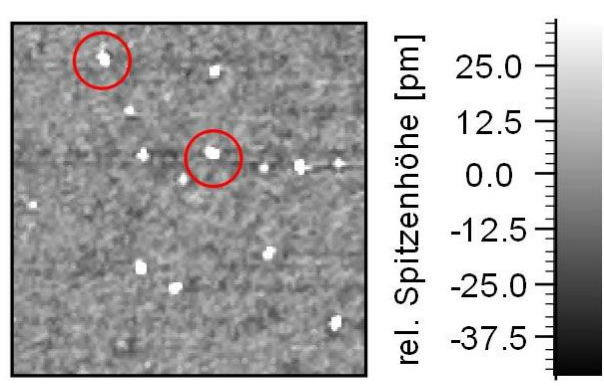

c)
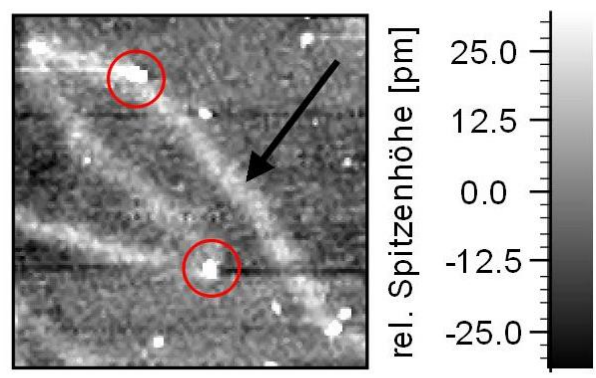

Abbildung 35: Langreichweitige Defekte auf zweilagigem Graphen durch Verspannungen. (a) Topographie von ein- und zweilagigem Graphen mit verschiedenen Stufenhöhen $\left(600 \times 600 \mathrm{~nm}^{2}, 512 \times 512\right.$ Pixel, $I_{\mathrm{T}}=0,3 \mathrm{nA}$, $U_{\text {Bias }}=60 \mathrm{mV}$ ). Vergrößerte Ausschnitte von $100 \times 100 \mathrm{~nm}^{2}$ sind in Abbildung (b) bzw. (c) dargestellt. Zweilagiges Graphen zeigt linienartige Erhöhungen, welche einige $10 \mathrm{pm}$ hoch sind und sich über mehrere $100 \mathrm{~nm}$ erstrecken können, die durch Faltung der oberen Graphenschicht entstehen. Dabei fallen lokalisierte Punktdefekte mit diesen räumlich zusammen ((c), rot markiert). Im Gegensatz dazu sind auf einlagigem Graphen in Abbildung (b) keine Falten zu beobachten.

Dabei sind auf beiden Lagen Punktdefekte lokalisiert, die in den STM-Topographien einige 100 pm hoch sind, wie in den vergrößerten Ausschnitten von einlagigem Graphen in Abbildung 35 (b) und zweilagigem Graphen in Abbildung 35 (c) durch rote Kreise markiert sind. Die atomare Struktur dieser Defekte ist unbekannt. Zusätzlich zu den Punktdefekten treten auf zweilagigem Graphen linienartige Defekte auf (siehe schwarze Pfeile), welche eine Höhe von einigen 10 pm besitzen und deren Orientierung und räumlicher Verlauf willkürlich erscheint (vgl. Abbildung 35 (a) und (b), schwarze Pfeile). Diese Falten der Graphenschicht werden engl. als Wrinkles bezeichnet. Ihr Zustandekommen wird dadurch erklärt, dass Graphen im Gegensatz zum darunterliegenden Substrat einen negativen thermischen Ausdehnungskoeffizienten besitzt. Beim Abkühlen des Substrates nach der 
Präparation wird die Graphenlage gestaucht, und es kommt zu einer Verspannung von bis zu 0,8 \% [88]. Es wird angenommen, dass die Antwort der ein- und zweilagigen Graphenschicht auf die Kompression dabei unterschiedlich ausfällt, da mit zunehmender Lagenanzahl die Zwischenschichtkopplung und damit die Kohäsionsenergie der Lagen untereinander abnimmt. Die Verspannungen werden dabei lokal über Punktdefekte in einlagigem Graphen und über die Wrinkles in zweilagigem Graphen abgebaut [89].

\subsubsection{Zusammenfassung}

Die in dieser Arbeit präparierten Proben weisen zahlreiche aus den letzten Jahren mit Hilfe der Rastertunnelmikroskopie ermittelte Eigenschaften von ein- wie auch zweilagigem Graphen auf. Es konnte anhand der spektroskopischen Analyse von einlagigem Graphen gezeigt werden, dass neben den bisherigen lokalisierten Zuständen weitere in der Nähe der Fermi-Energie existieren und besonders auch bei $E_{\mathrm{F}}$ Variationen in der differentiellen Leitfähigkeit sichtbar sind. Für zweilagiges Graphen konnte anhand der Spektroskopie der Dirac-Punkt, die Bandlücke sowie das Einsetzten des zweiten Leitungsbandes bestimmt werden. Offen bleibt die Frage nach der genauen Struktur des Interfaces. Die beobachtete Defektdichte innerhalb der Zwischenschicht liefert unter der Annahme, dass diese als Donatoren für das epitaktische Graphen wirken, eine plausible Ladungsträgerkonzentration der epitaktisch gewachsenen Graphenschicht.

Im Rahmen der Doktorarbeit hat sich gezeigt, dass die spektroskopische Charakterisierung im Vergleich zu anderen in der Arbeitsgruppe verwendeten Probensystemen wie GaAs(110) oder $\mathrm{Cu}(111)$ eine niedrigere energetische und räumliche Auflösung hat. Eine mögliche Ursache hierfür können die beobachteten Oberflächenmodifikationen sein, welche sich scheinbar in der Zwischenschicht abspielen. Neben den in der Zwischenschicht meist als Trimere erscheinenden lokalisierten Defekten, treten auf ein- und zweilagigem Graphen auch Punktdefekte auf, die sich durch eine größere rel. Spitzenhöhenänderung auszeichnen und eine geringere Defektdichte besitzen. Aufgrund der beim Wachstum auftretenden Kompression der ein- und zweilagigen Graphenschicht kommt es auf zweilagigem Graphen zur Faltenbildung, den sogenannten Wrinkles. 


\section{Thermospannung auf $\mathrm{Cu}(111)$ sowie epitaktischem Graphen}

\subsection{Einfluss der Thermospannung auf Tieftemperatur-Potentiometrie- Messungen}

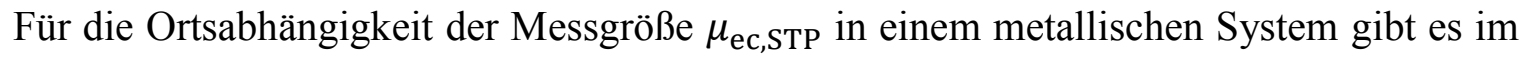
Allgemeinen zwei grundlegende Ursachen: zum einen eine Nicht-Gleichgewichtsverteilung des elektrochemischen Potentials der Probe hervorgerufen durch ein laterales Transportfeld, wie in Kapitel 2.1.2 diskutiert worden ist, und zum anderen eine Temperaturdifferenz zwischen Spitze und Probe, d.h. Spitze und Probe befinden sich nicht im thermischen Gleichgewicht. Im letzteren Fall führt die Temperaturdifferenz zwischen Spitze und Probe zu einer messbaren Thermospannung am Tunnelkontakt bei verschwindendem Tunnelstrom in Analogie zum klassischen Seebeck-Effekt.

Um Potentiometriedaten eines Transportexperiments richtig interpretieren zu können, ist es daher unerlässlich, beide Ursachen voneinander unterscheiden zu können. In diesem Kapitel soll zunächst eine kurze Einleitung über bisherige theoretische und experimentelle Arbeiten zur Thermospannung am Tunnelkontakt gegeben werden. Im Rahmen dieser Doktorarbeit hat sich gezeigt, dass das hier verwendete Tieftemperatur-STM konstruktionsbedingt bei 6 K einen Temperaturunterschied zwischen Spitze und Probe besitzt. Das Auftreten der Thermospannung wird durch Messungen auf einem Cu(111)-Einkristall demonstriert und dessen Eigenschaften diskutiert. Die $\mathrm{Cu}(111)$-Oberfläche wurde gewählt, da diese unter anderem mit dem STM in Bezug auf seine elektronischen Eigenschaften intensiv untersucht worden ist [90,91]. Insbesondere wird gezeigt, wie die Thermospannung aus bestehenden Datensätzen minimiert werden kann und wo die Grenzen dieses Verfahrens liegen. Im Anschluss werden Thermospannungssignaturen auf den SiCGraphen-Proben analysiert und mit Hilfe der Thermospannungssignaturen dominierende Streumechanismen auf ein- und zweilagigem Graphen diskutiert. 


\subsubsection{Thermospannung am Tunnelkontakt}

Der erste der drei thermoelektrischen Effekte (Seebeck-, Pelletier- und Thomson-Effekt) wurde von Seebeck im Jahr 1821 an einem geschlossenen Stromkreis, welcher aus zwei unterschiedlichen Metallen zusammengesetzt war, beobachtet $[92,93]$. Besitzen die Kontaktstellen verschiedene Temperaturen $T_{1}$ und $T_{2}$, kommt es zu einem Diffusionsstrom innerhalb des Stromkreises, welcher dem Temperaturgradienten entgegenwirkt. Ist der Stromkreis geöffnet, baut sich durch entsprechende Ladungsanhäufung entlang des Stromkreises ein elektrisches Feld, das sogenannte Thomson-Feld auf, welches die Diffusion der thermisch angeregten Ladungsträger kompensiert. Die daraus resultierende Thermospannung $U_{\text {Th }}$ kann an den Enden des Leiters abgegriffen werden. Die Änderung der Thermospannung mit sich verändernder Temperatur wird als Thermokraft oder Seebeck-Koeffizient bezeichnet und ist für eine bestimmte Temperatur als $S=\lim _{d T \rightarrow 0} \frac{d U_{T h}}{d T}$ definiert. Die Größe des Seebeck-Koeffizienten und dessen Temperaturabhängigkeit ist direkt gekoppelt an die elektrische Leitfähigkeit. Das Konzept der Thermospannung lässt sich ebenso für einmetallische Punktkontakte [94] wie auch Tunnelbarrieren übertragen [95]. Die Thermospannung im Tunnelkontakt eines STM wurde bisher theoretisch von C. R. Leavens und G. C. Aers [96] sowie J.A. Støvneng und P. Lipavský [97] behandelt. Einen einfachen analytischen Zugang zur Thermospannung erhält man über die Theorie von J.A. Støvneng und P. Lipavský welche auf der Tunneltheorie von Tersoff und Hamann basiert.

Für die Thermospannung erhalten J. A. Støvneng und P. Lipavský unter der Annahme einer linearen Zustandsdichte der Spitze und Probe bei der Fermi-Energie folgenden Ausdruck:

$$
U_{\mathrm{Th}}=\frac{\pi^{2} \cdot k_{B}^{2}}{6 \cdot e}\left(T_{\mathrm{S}}^{2}-T_{\mathrm{P}}^{2}\right)\left(\left.\frac{1}{\rho_{\mathrm{S}}} \frac{\partial \rho_{S}}{\partial E}\right|_{E_{\mathrm{F}}}+\left.\frac{1}{\rho_{\mathrm{P}}} \frac{\partial \rho_{\mathrm{P}}(\mathbf{r})}{\partial E}\right|_{E_{\mathrm{F}, z=0}}+\frac{z}{\hbar} \sqrt{\frac{2 \cdot m}{\phi}}\right)
$$

mit den Temperaturen von Spitze und Probe $\mathrm{T}_{\mathrm{S}}$ und $\mathrm{T}_{\mathrm{P}}$, deren lokaler Zustandsdichte $\rho_{S}$ und $\rho_{P}$, dem Tunnelabstand $z$ sowie der effektiven Barrierenhöhe $\phi$ [97]. Anhand von Gleichung 4.1 lassen sich grundlegende Eigenschaften der Thermospannung ablesen. So ist die Thermospannung unter anderem sensitiv auf lokale Änderungen der Zustandsdichte der Probe bei der Fermi-Energie bei ansonsten festgehaltenen Parametern. Dies wurde erstmals 
mit atomar aufgelösten Thermospannungsmessungen am Tunnelkontakt von Weaver, Williams und Wickramasinghe an geheizten Gold und $\mathrm{MoS}_{2}$-Substraten demonstriert [98-100]. Unter anderem konnte die chemische Sensitivität der Methode durch die unterschiedlichen Beiträge der Thermospannung innerhalb einer Einheitszelle des $\mathrm{MoS}_{2}$ gezeigt werden. Darauf folgende Arbeiten bestimmten unter anderem den Seebeck-Koeffizienten zwischen heterogenen Metalloberflächen wie $\mathrm{Ag}(111)$ auf $\mathrm{Au}(111)$ oder Pb auf $\mathrm{Au}(111)$ [101-103]. Aufgrund der hohen lateralen Auflösung im Subångström-Bereich und energetischen Auflösung von einigen $\mu \mathrm{V}$ eignet sich die Methode der Thermospannungs- Rastertunnelpotentiometrie ebenso, um das Streuverhalten von Elektronen bei der Fermi-Energie an Defekten zu studieren, wie es am Beispiel von Goldstufen und Oberflächenrekonstruktionen der $\mathrm{Au}-$ und $\mathrm{Cu}(111)$ Oberfläche demonstriert worden ist [104-106].

Nach Gleichung 4.1 ist die Thermospannung ebenso linear abhängig vom Abstand zwischen Spitze und Probe. Dieser Zusammenhang konnte bisher nur grob an Thermospannungsmessungen auf $\mathrm{Au}(111)$ bei Raumtemperatur und einer Temperaturdifferenz von $15 \mathrm{~K}$ zwischen Spitze und Probe sowie einer relativen Abstandsänderung von bis zu 300 pm evaluiert werden [106].

\subsubsection{Charakterisierung der Thermospannung auf $\mathrm{Cu}(111)$}

Um die Thermospannung am Tunnelkontakt des verwendeten Tieftemperatur-STMs zu bestimmen wurden Potentiometriemessungen an der $\mathrm{Cu}(111)$-Oberfläche vorgenommen. Diese bildet durch einen Shockley-Oberflächenzustand ein zweidimensionales Elektronengas an der Oberfläche mit einer parabolischen Dispersionsrelation. Die Leitungsbandkante liegt $440 \mathrm{meV}$ unterhalb von $\mathrm{E}_{\mathrm{F}}$ mit einer effektiven Masse von $m^{*}=0,38 \cdot m_{0}[91,107]$. Die Fermi-Wellenlänge beträgt $30 \AA$. Abbildung 36 (a) zeigt eine Topographie der $\mathrm{Cu}(111)$ Oberfläche mit einer monoatomaren Stufe. An solchen Stufen und Punktdefekten kommt es zur Streuung der Elektronen des Oberflächenzustandes und damit zu periodischen Oszillationen in der lokalen Zustandsdichte, den sogenannten Friedel-Oszillationen. Diese werden für kleine Spannungen von der Topographie abgebildet, da in erster Näherung angenommen werden kann, dass die Topographieabbildung proportional zur Änderung der lokalen Zustandsdichte ist. 

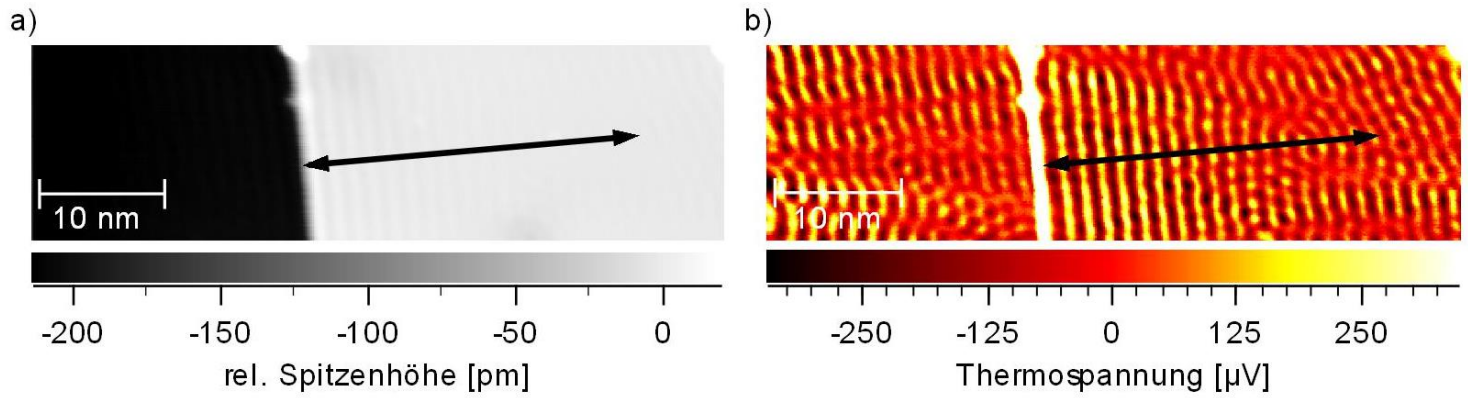

c)

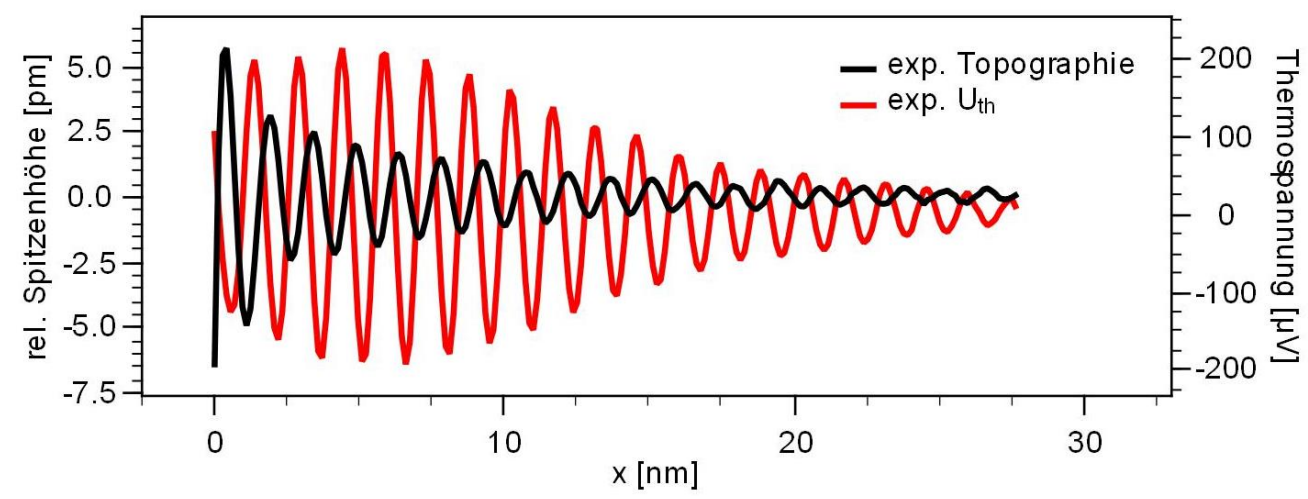

Abbildung 36: (a) Topografie einer $\mathrm{Cu}(111)$-Oberfläche mit monoatomarer Stufe. Aufgrund der Streuung der Elektronen an dem Defekt kommt es zu Modulationen in der lokalen Zustandsdichte, sogenannten Frideloszillationen. (b) Simultan aufgenommene Thermospannung mit Hilfe der Rastertunnel-potentiometrie. Die Thermospannung ist proportional zur Ableitung der lokalen Zustandsdichte nach der Energie bei $E_{\mathrm{F} .}$ (c) Lateraler Schnitt entlang des in (a) und (b) markierten Bereiches.

Die Periodizität der beobachteten Wellenmuster beträgt $15 \AA$ und entspricht damit der halben Fermi-Wellenlänge von $\mathrm{Cu}(111)$. Abbildung 36 (b) zeigt die zur Topographie simultan mit Hilfe der RTP aufgenommene Thermospannung. Diese liegt in der Größenordnung von einigen $100 \mu \mathrm{V}$ und zeigt die charakteristischen Merkmale der Thermospannung. Ein direkter Vergleich der Oszillationen in Abbildung 36 (c) zeigt, dass die Oszillationen im Thermospannungssignal phasenverschoben zu denen in der Topographie sind und Ihre maximale Amplitude von ca. $200 \mu \mathrm{V}$ erst im Abstand von einigen Nanometern erreicht wird. Dies wurde ebenso auf der $\mathrm{Au}(111)$-Oberfläche beobachtet [101, 104].

Das gemessene Thermospannungssignal ist ein direkter Beweis, dass sich Spitze und Probe nicht im thermischen Gleichgewicht befinden können. Dabei ist durch die Konstruktion der Anlage anzunehmen, dass die Spitze eine wesentlich höhere Temperatur besitzt als die Probe. Die Probentemperatur konnte durch ein an der Probenaufnahme montiertes Thermoelement auf 5,6(1) $\mathrm{K}$ bestimmt werden und hat folglich eine gute thermische Ankopplung an den Helium-Kryostaten. Im Gegensatz dazu besteht eine thermische Verbindung 
zum Heliumkryostaten am STM-Kopf nur über einige Silberhaare, welche auf eine wiederum thermisch schlecht leitfähige Edelstahlhalterung am STM-Kopf montiert sind. Daher ist davon auszugehen, dass sich die Spitze durch die wesentlich stärker erwärmt als die Probe, da durch die optischen Zugänge Wärmestrahlung eindringt.

Um die Temperaturdifferenz zwischen Spitze und Probe nach der auf Tersoff und Hamann basierenden Theorie für die Thermospannung nach Gleichung 4.1 aus den gezeigten experimentellen Daten abzuschätzen, wird die lokale Barrierenhöhe, sowie die orts- und energieabhängige lokale Zustandsdichte der Probe benötigt. Die Zustandsdichte der Spitze wird als konstant angenommen. Die lokale Zustandsdichte der Probe $\rho_{P}$ an einer Stufe kann durch den Ausdruck

$$
\begin{gathered}
\rho_{\mathrm{P}}(E, x, y, z)=\frac{m^{*}}{\pi \hbar^{2}} e^{-x / l_{\varphi}}\left(1-R J_{0}\left(2 k_{E} x\right)\right) e^{-2 \alpha(E) z} \\
\operatorname{mit} \alpha(E)=\frac{1}{\hbar} \sqrt{2 m_{0}(\phi-E)+\hbar^{2} k_{\|}} \text {und } k_{E}=\frac{1}{\hbar} \sqrt{2 m^{*}\left(E-E_{\Gamma}\right)}
\end{gathered}
$$

beschrieben werden [90, 91, 108]. Dabei ist R die Reflektivität der Stufe, J $\mathrm{J}_{0}$ die Besselfunktion 0 -ter Ordnung und $l_{\varphi}$ die Phasenkohärenzlänge. Die lokale Barrierenhöhe wurde durch Topographien am selben Ort zu verschiedenen Stromsetzwerten bei der in Abbildung 36 (a) gezeigten Stufe bestimmt und beträgt 3,8(1) eV. Für die Phasenkohärenzlänge wurde der Wert $l_{\varphi}=33 \mathrm{~nm}$ gewählt $[108,109]$. Um die Topographie zu simulieren wurde die relative Spitzenhöhenänderung senkrecht zur Stufe nach Gleichung 2.8 berechnet, indem der experimentell verwendete Setzstrom von 0,6 nA auf einen für Metalle typischen Tunnelabstand von $0,5 \mathrm{~nm}$ normiert worden ist. Die beste Übereinstimmung zwischen den theoretisch berechneten und experimentellen Daten wurde für eine Reflektivität der Stufenkante von $R=0,35$ erzielt. Im nächsten Schritt wurde der Thermostrom für verschwindende Bias-Spannung bei gegebenem Spitzen-Probenabstand und die dazugehörige Thermospannung unter der Bedingung $I_{\mathrm{T}}\left(U_{\mathrm{Th}}, T_{\mathrm{S}}, T_{\mathrm{P}}\right)=0$ nach Gleichung 2.9 für verschiedene Temperaturen berechnet. Dies hat zur Folge, dass der Verlauf der theoretisch berechneten Thermospannung nicht exakt dem experimentellen Verlauf entsprechen kann.

Abbildung 37 (a) zeigt die anhand der gewählten Parameter berechnete Topographie und das zugehörige Thermospannungssignal für eine Spitzentemperatur von $108 \mathrm{~K}$. Ein direkter quantitativer Vergleich mit den experimentellen Daten ist an dieser Stelle nur eingeschränkt 
möglich, da die lokale Zustandsdichte an der Stufenkante durch umliegende Defekte und Adsorbate gestört ist, und es somit im Vergleich zum theoretischen Fall einer ungestörten, unendlich ausgedehnten Stufe zu zusätzlichen Interferenzen kommt.

a)

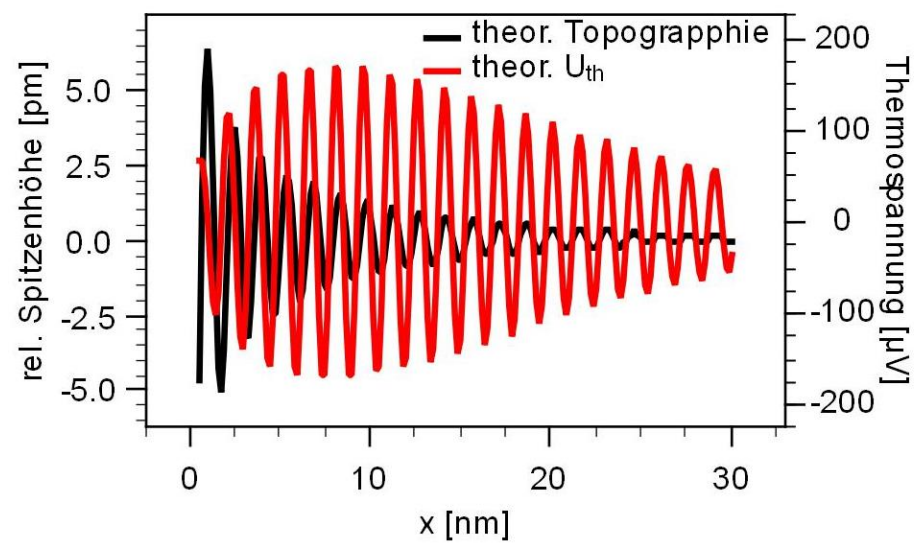

b)

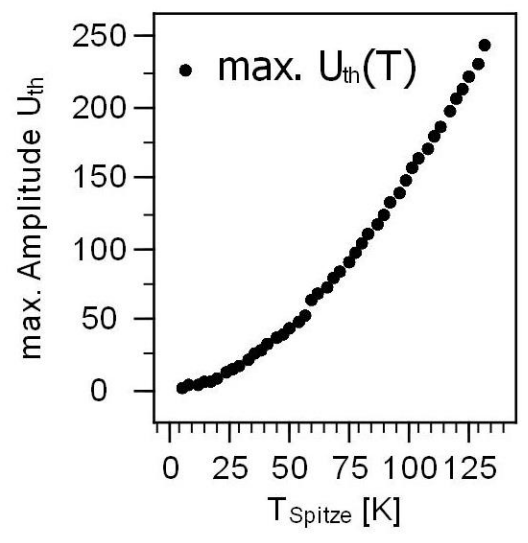

Abbildung 37: (a) Theoretisch berechnete Topographie und Thermospannung für eine unendlich ausgedehnte monoatomare $\mathrm{Cu}(111)$-Stufe $\left(R=0,35, l_{\varphi}=33 \mathrm{~nm}, T_{\mathrm{P}}=5,6 \mathrm{~K}\right)$. Charakteristische Merkmale, wie der Phasenschub der Thermospannungsoszillationen zu denen in der Topographie sowie die Einhüllende der Thermospannung, sind mit den experimentellen Daten (vgl. Abbildung 36 (c)) gut vergleichbar. (b) Berechnete maximale Amplitude der Thermospannungsoszillationen in der Umgebung der Stufe in Abhängigkeit von der Spitzentemperatur $\mathrm{T}_{\mathrm{S}}$ und Parametern aus (a).

Abbildung 37 (b) zeigt die berechnete maximale Thermospannungsamplitude in Abhängigkeit von der Spitzentemperatur bei einer Probentemperatur von 5,6(1) K. Vergleicht man die experimentell auftretenden Amplituden von 100 bis $200 \mu \mathrm{V}$ mit den theoretisch berechneten, ist nach diesem Modell für die Thermospannung eine Temperaturdifferenz in der Größenordnung von einigen $10 \mathrm{~K}$ bis $100 \mathrm{~K} \mathrm{zu}$ erwarten. Eine genauere Bestimmung der Temperaturdifferenz zwischen Spitze und Probe mit Hilfe von lateral aufgelösten Thermospannungsmessungen ist prinzipiell möglich, erfordert jedoch größere defektfreie Terrassen und Stufenkanten, so dass diese dem theoretischen Modell einer perfekten monoatomaren Stufe besser entsprechen. 


\subsection{Thermospannungssignaturen auf Graphen}

In diesem Kapitel soll auf die Eigenschaften der Thermospannung auf ein- und zweilagigem Graphen eingegangen werden. Abbildung 38 (a) zeigt einen $5 \times 5 \mathrm{~nm}^{2}$ großen Bereich von einlagigem Graphen, aufgenommen bei RT. Deutlich zu erkennen sind die atomare Korrugation des Graphengitters sowie die durch die Zwischenschicht hervorgerufene 6×6Überstruktur. Abbildung 38 (b) zeigt die dazugehörigen Potentialdaten. Diese sind zur Topographie unkorreliert und zeigen keinerlei Korrugation in Bezug auf das Graphengitter. Dies steht mit der Theorie im Einklang, dass im thermodynamischen Gleichgewicht das lokale elektrochemische Potential räumlich nicht variiert.

a)

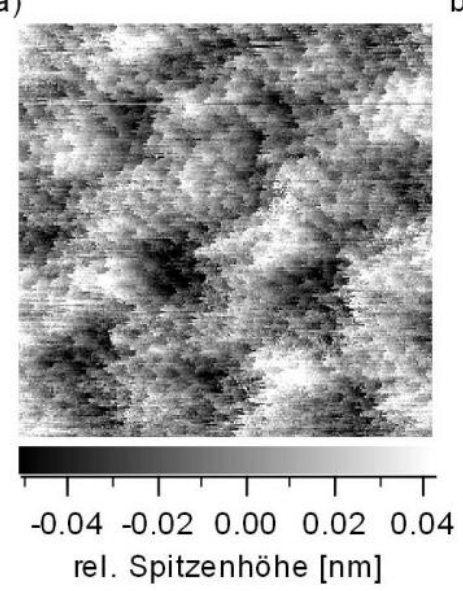

b)

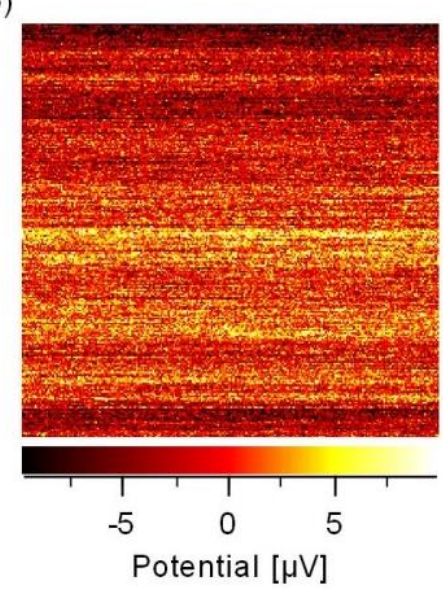

c)

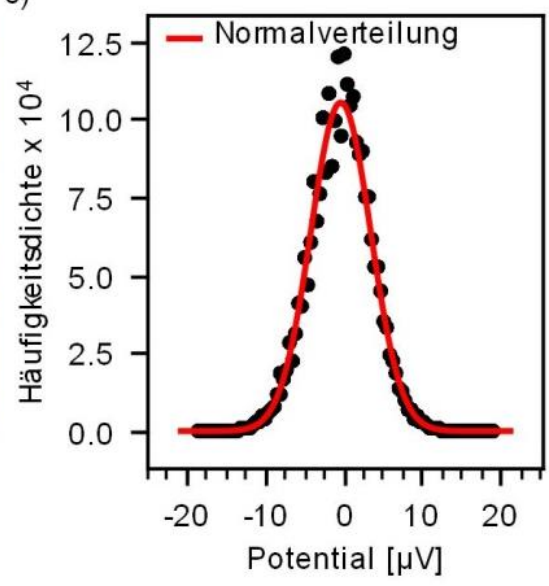

Abbildung 38: Potentiometrie Datensatz von einlagigem Graphen, aufgenommen bei RT. (a) Topographie und (b) Potentiometriedaten bei Raumtemperatur zeigen keine Korrelationen. (c) Die Potentialwerte sind normalverteilt mit einer Standardabweichung von $3,7 \mu \mathrm{V}\left(5 \times 5 \mathrm{~nm}^{2}, U_{\mathrm{Bias}}=10 \mathrm{mV}\right.$ und $\left.I_{\mathrm{T}}=600 \mathrm{pA}\right)$.

Die langwelligen Modulationen im Potentiometrie-Signal entlang der langsamen Rasterrichtung im Bereich von einigen $\mu \mathrm{V}$ werden von Potentialschwankungen der Elektronik verursacht. Die bestimmten Potentialwerte sind normalverteilt und besitzen eine Standardabweichung von 3,7 $\mu \mathrm{V}$. Dieser Wert liegt bei einem gegebenen Tunnelwiderstand von $R_{\mathrm{T}}=16,7 \mathrm{M} \Omega$ und einer gegebenen Mittelungszeit von $33 \mathrm{~ms}$ für jeden einzelnen Potentialmesswert nur etwa $30 \%$ über dem theoretisch erwarteten thermischen Rauschen des Tunnelkontaktes (siehe Kapitel 2.1.3). Ein nahezu identisches Verhalten wurde ebenso auf der metallischen $\operatorname{Si}(111) \sqrt{3} \times \sqrt{3}-$ Ag Rekonstruktion gefunden [34]. Aus den Beobachtungen lässt sich schließen, dass der Temperaturunterschied zwischen Spitze und Probe bei Raumtemperatur aufgrund der nicht auftretenden Thermospannung vernachlässigbar ist. 
Zum Vergleich ist in Abbildung 39 (a) und (b) sowohl ein Potentiometriedatensatz einlagigen Graphens sowie in Abbildung 39 (d) und (e) zweilagigen Graphens und deren Potentialverteilung in (c) und (e) gezeigt, welche bei $6 \mathrm{~K}$ Probentemperatur aufgenommen worden sind.

a)

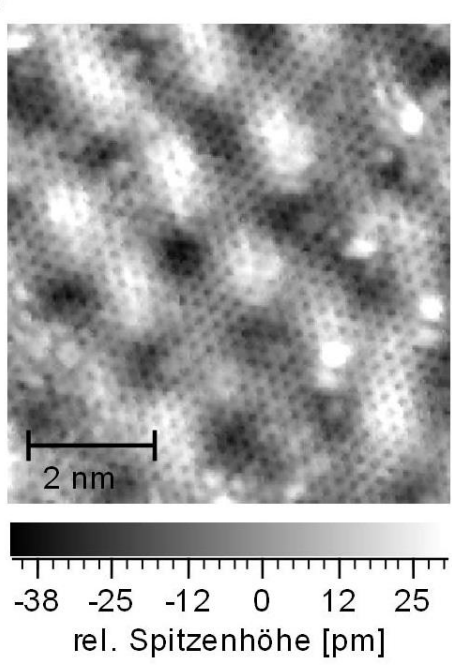

d)

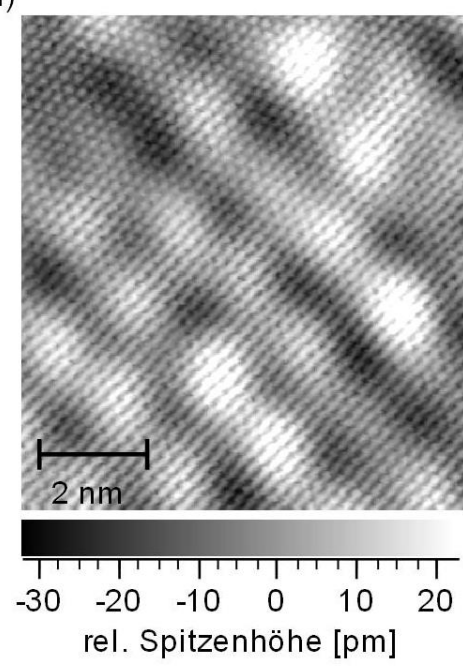

b)

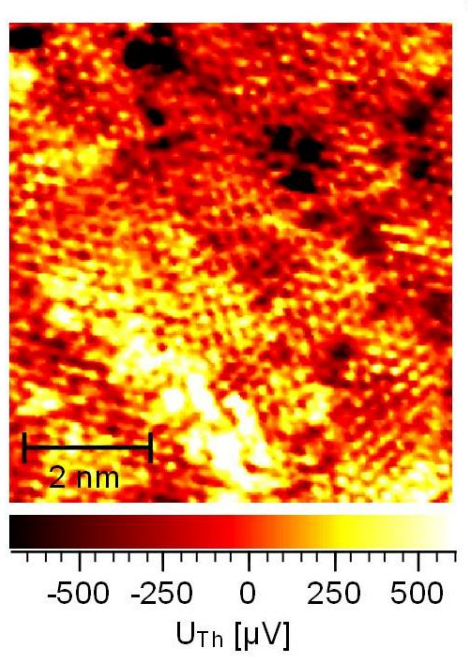

e)

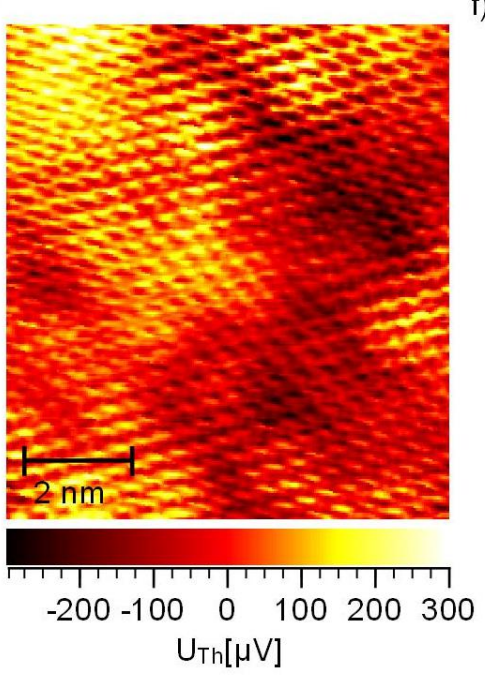

c)

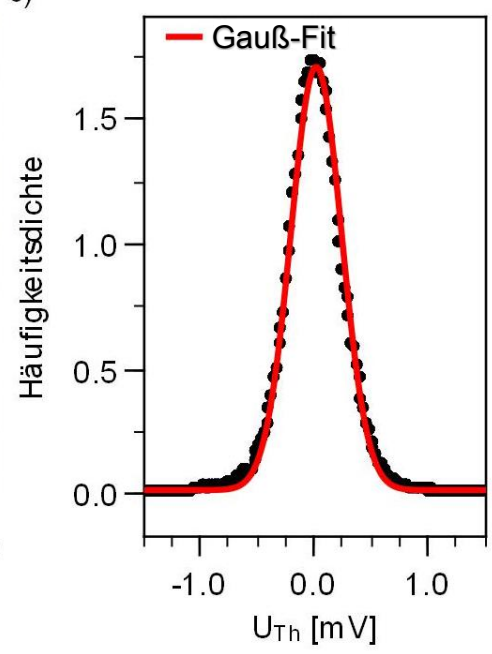

f)

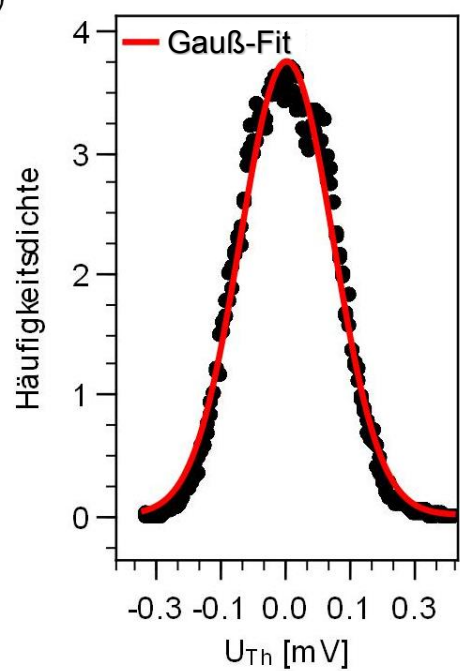

Abbildung 39: Vergleich der Thermospannungssignaturen zwischen ein- und zweilagigem Graphen bei $6 \mathrm{~K}$. Topographie- und Thermospannungsdaten sowie Verteilung der Potentialwerte sind in (a) bis (c) für einlagiges Graphen und (d) bis (f) für zweilagiges Graphen dargestellt $\left(I_{\mathrm{T}}=0,24 \mathrm{nA}, U_{\text {Bias }}=-60 \mathrm{mV}\right)$. Laterale- sowie Amplituden-Variationen in $U_{\text {Th }}$ sind auf einlagigem Graphen höher als auf zweilagigem Graphen. Die Halbwertsbreiten der angepassten Gauß-Kurven betragen für die hier gezeigten Bilder $530 \mu \mathrm{V}$ für einlagiges und $256 \mu \mathrm{V}$ für zweilagiges Graphen.

Die Topographien (vgl. Abbildung 39 (a) und (d)) zeigen wiederum die charakteristischen topographischen Merkmale von ein und zweilagigem Graphen, wie sie in Kapitel 3.5 beschrieben worden sind. Im Gegensatz zu den Raumtemperaturdaten zeigt sich eine klare 
räumliche Korrelation zwischen gemessener Topographie und lokal variierenden Potentialwerten in der Größenordnung von bis zu einigen $100 \mu \mathrm{V}$. Die Ursache hierfür ist der Temperaturgradient im Tunnelkontakt und die damit verbundene auftretende Thermospannung. Daher ist es möglich, mit dem Verfahren der Rastertunnelpotentiometrie auf ein- und zweilagigem Graphen direkt Änderungen der Ableitung der lokalen Zustandsdichte der Probe bei $E_{\mathrm{F}}$ ortsaufgelöst zu bestimmen. Aus diesem Grund sind in den Thermospannungsdaten periodische Modulationen mit identischen Wellenlängen wie in der Topographie sichtbar. Die lateralen Variationen in der lokalen Zustandsdichte bzw. deren Ableitung ergeben sich aus einer Überlagerung der elektronischen Struktur der Oberfläche, wie dem Graphengitter und der Streuung von Elektronen an Defekten.

Vernachlässigt man die Symmetrieeigenschaften der Wellenfunktionen für ein- und zweilagiges Graphen, gibt es zwei grundlegende mögliche elastische Streumechanismen auf diesen, welche im Folgenden in Anlehnung an die Arbeiten von unter anderem Mallet und Brihuega $[86,110]$ diskutiert werden sollen, die sog. Intervalley- und Intravallay-Streuung. In Abbildung 40 (a) ist ein zweidimensionaler Schnitt durch die Fermifläche sowie die zweidimensionale Brillouinzone dargestellt. Vernachlässigt man Effekte wie Trigonal Wrapping [86], besitzt sowohl ein- wie auch zweilagiges n-dotiertes Graphen um die K bzw. K'-Punkte jeweils einen isotropen Fermi-Ring mit dem Radius $\left|\mathbf{q}_{\mathbf{F}}\right|$. Der Vektor $\mathbf{q}_{\mathbf{F}}$ bezeichnet den Fermiwellenvektor, gemessen vom Mittelpunkt eines beliebigen K-Punktes. Die Vektoren k1 und k2 bezeichnen Wellenvektoren eines Ausgangs- und eines Endzustands eines gestreuten Elektrons. Bei der Intravalley-Streuung (vgl. Abbildung 40 (c)) werden Elektronen innerhalb eines Konus bzw. Fermi-Rings gestreut. Nimmt man an, dass die Streurate für gegenüberliegende Zustände mit $\mathbf{k}_{\mathbf{1}}=\mathbf{q}_{\mathbf{F}}$ und $\mathbf{k}_{\mathbf{2}}=-\mathbf{q}_{\mathbf{F}}$ auf der Fermifläche dominieren, wie das für normale zweidimensionale Elektronengase der Fall ist [111], entspricht der Betrag des Streuvektors gerade dem doppeltem Fermiwellenvektor, also |k2$\mathbf{k}_{1}|=2| \mathbf{q F}_{\mathbf{F}} \mid$. In der fouriertransformierten LDOS erwartet man daher einen Ring mit dem Radius $\left|2 \mathbf{q F}_{\mathbf{F}}\right|$ im Zentrum der Brillouin-Zone und im Realraum langwellige Modulationen mit der Fermiwellenlänge $\lambda_{\mathrm{F}} / 2$.

Bei der Intervalley-Streuung werden Elektronen von einem Fermi-Ring in den benachbarten gestreut, wie z.B. von $\mathrm{K}_{1}$ nach $\mathrm{K}_{2}{ }^{`}$ (vgl. Abbildung 40 (b)). 
a)

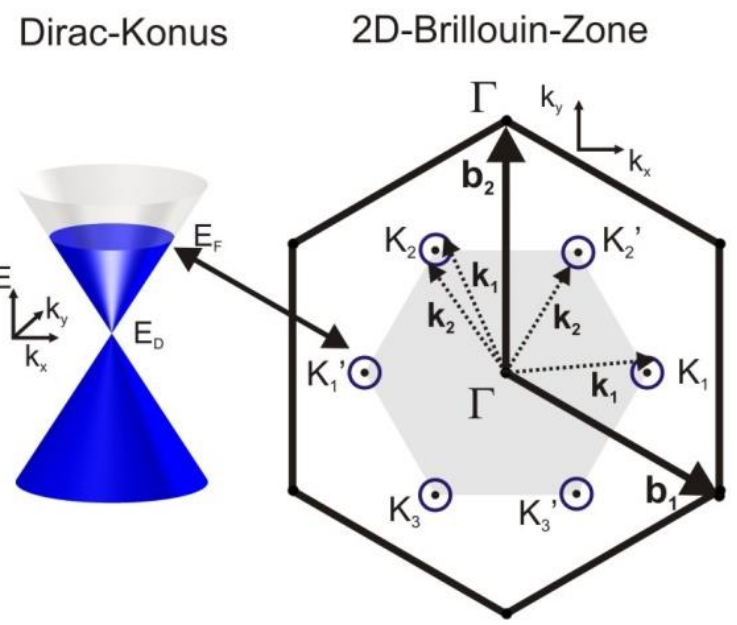

b)

Intervalley-Streuung Intravalley-Streuung

c)

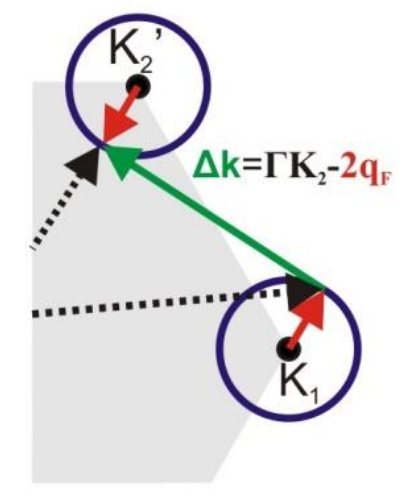

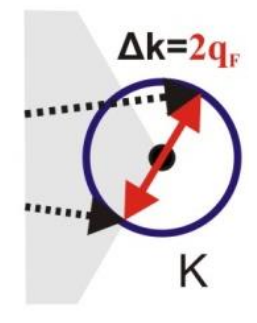

Abbildung 40: Prinzipielle elastische Streumechanismen in ein- und zweilagigem Graphen. (a) Schematische Darstellung der Dispersionsrelation von n-dotiertem, einlagigem Graphen um einen der K-Punkte sowie die 2d-Brillouin-Zone. Iso-Energieschnitte durch die Fermifläche bei den K-Punkten ergeben isotrope Ringe. Die Vektoren $\mathbf{k}_{\mathbf{1}}$ und $\mathbf{k}_{\mathbf{2}}$ bezeichnen Wellenvektoren vom ungestreuten bzw. gestreuten Elektron. (b) Streuprozess zwischen zwei Fermi-Ringen wird als Intervalley-Streuung bezeichnet. (c) Streuprozess innerhalb eines Fermirings bzw. Konus wird Intravalley-Streuung genannt.

Die Differenz der Wellenvektoren von Ausgans- und Endzustand beträgt in diesem Fall $\Delta \mathbf{k}=\Gamma \mathbf{K}_{\mathbf{2}}-2 \mathbf{q}_{\mathbf{F}}$. Allgemein erhält man somit durch die Intervalley-Streuung Streuvektoren, deren Länge nahezu den reziproken Gittervektoren $\mathbf{K}$ bzw. $\mathbf{K}^{`}$ entsprechen [60], so dass man im Realraum durch Streuung an Oberflächendefekten oder Graphenrändern eine $\sqrt{3} \times \sqrt{ } 3 \mathrm{R} 30^{\circ}$-Überstruktur erhält. Berücksichtigt man nun die Symmetrieeigenschaften der Wellenfunktionen in ein- und zweilagigem Graphen, so ist in einlagigem Graphen im Gegensatz zu zweilagigem Graphen die Intravalley-Streuung aufgrund der Pseudospinerhaltung unterdrückt. Die Intervalley-Streuung hingegen ist für beide Systeme zulässig [86, 110].

Abbildung 41 zeigt die fouriertransformierten Topographien und Potentialdaten aus Abbildung 39. In den FFT-Darstellungen der Topographien von einlagigem wie auch zweilagigem Graphen finden sich Intensitätsmaxima, welche hexagonal angeordnet sind und der Länge des reziproken Gittervektors des Graphengitters von $\left|\mathbf{b}_{1,2}\right|=29,5 \mathrm{~nm}^{-1}$ entsprechen. In den FFT-Darstellungen der Thermospannungsdaten sind neben diesen ebenso Spots der $(\sqrt{3} \times \sqrt{3}) \mathrm{R} 30^{\circ}$-Überstruktur erkennbar, welche durch die Intervalley- Streuung der Elektro- 
nen an lokalisierten Oberflächendefekten auf ein- und zweilagigem Graphen zustande kommen. Mit Hilfe der Thermospannungsdaten erhält man somit auf ein- und zweilagigem Graphen Signaturen, die vergleichbar sind mit denen von Mallet et al. Dort wurden die Streueigenschaften auf ein- und zweilagigem epitaktischem Graphen durch die Messungen der differentiellen Leitfähigkeit und damit der lokalen Zustandsdichte bei verschiedenen Energien untersucht. Der Unterschied besteht jedoch darin, dass das Thermospannungssignal zur Ableitung der LDOS bei $E_{\mathrm{F}}$ proportional ist.

a)

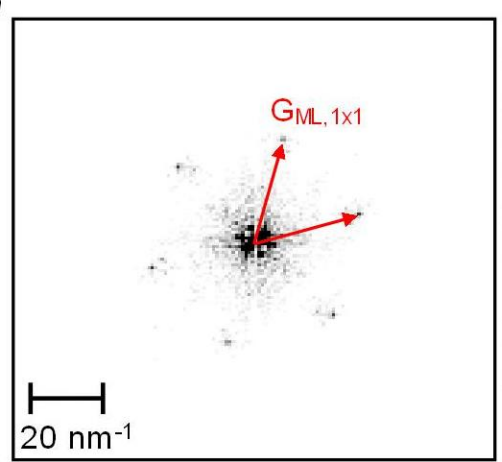

c)

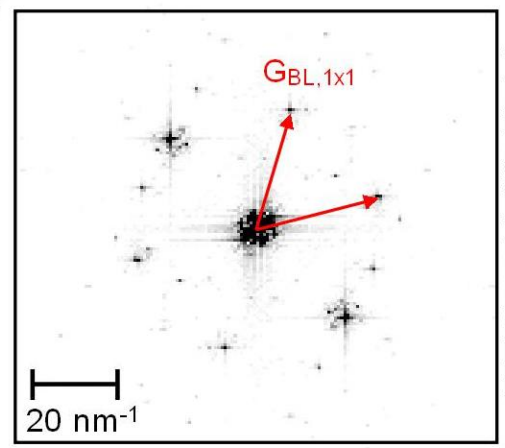

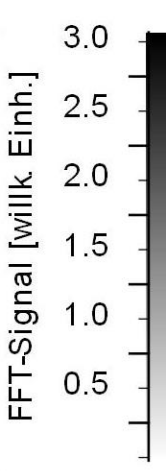

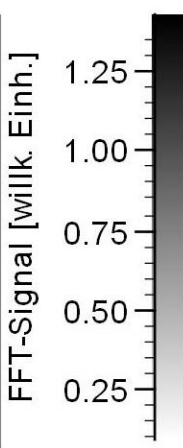

b )
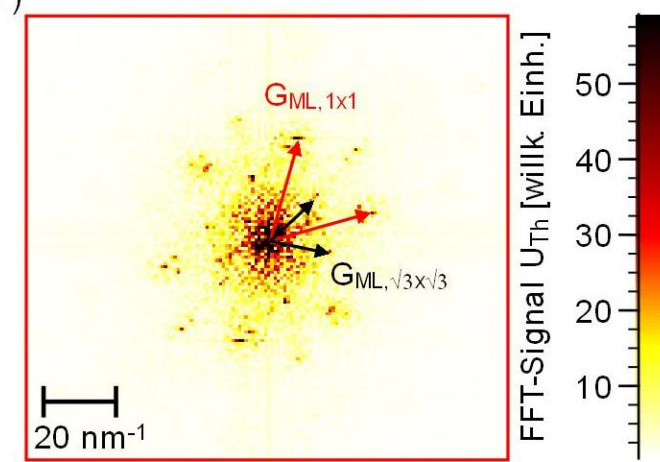

d)

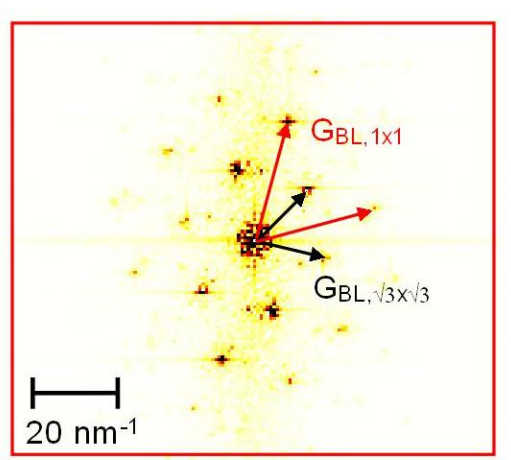

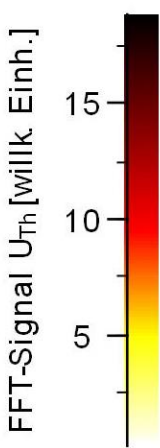

Abbildung 41: Vergleich der fouriertransformierten Topographie- und Thermospannungssignaturen von einlagigem Graphen (a, b) und zweilagigem Graphen (c, d) aus Abbildung 39. In der FFT der Topographien sind die Spots des Graphengitters zu erkennen. Zusätzlich zu diesen sind in den Thermospannungsdaten die $(\sqrt{3} \times \sqrt{3}) \mathrm{R} 30^{\circ}$-Überstruktur zu erkennen, welche durch Intervalley Streuung an Defekten zustande kommt.

Aus Abbildung 39 (b) ist ebenso erkennbar, dass Defektstrukturen, welche der Zwischenschicht zugeschrieben werden, einen besonders starken Kontrast in der Thermospannung hervorrufen und ebenso stark lokalisierte und erhöhte Kontraste an Orten vorliegen, die vermeidlich in der dazugehörigen Topographie keine Defekte erkennen lassen. Im Allgemeinen treten diese stark lokalisierten Änderungen in der Thermospannung häufiger auf einlagigem als auf zweilagigem Graphen auf. Dies ist wiederum ein Indiz, dass einlagiges 
epitaktisches Graphen elektronisch wesentlich stärker durch die darunterliegende Zwischenschicht beeinflusst wird als zweilagiges Graphen.

Neben der Intervalley-Streuung kann ebenso die Intravalley-Streuung auf zweilagigem Graphen in den Thermospannungsdaten beobachtet werden. Abbildung 42 (a) zeigt einen $90 \times 82 \mathrm{~nm}^{2}$ großen Topographieausschnitt einer zweilagigen Graphenschicht, das dazugehörige gemessene Thermospannungssignal in (b) sowie das fouriertransformierte Signal der Thermospannung in Abbildung (c) aus dem oberen Bereich in (b). In der Topographie ist im unteren Teil des Bildes eine topographische Erhöhung von 200 bis 300 pm zu erkennen. Dabei handelt es sich um eine stark ausgeprägte, wie in Kapitel 3.5.3 beschriebene Falte bzw. Wrinkle in der oberen Graphenlage.

a)

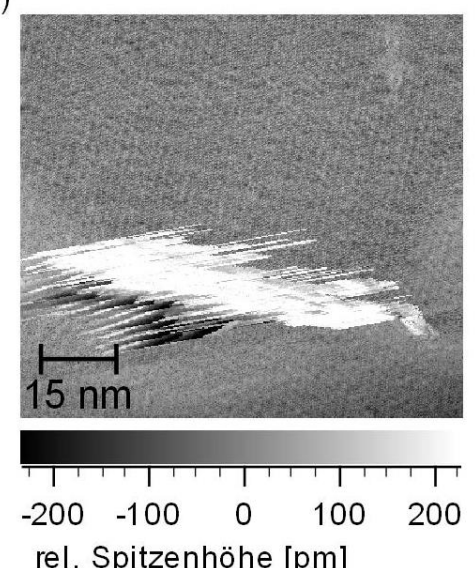

b)

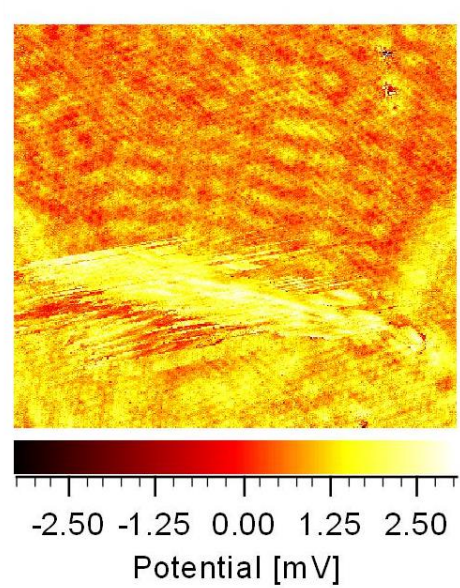

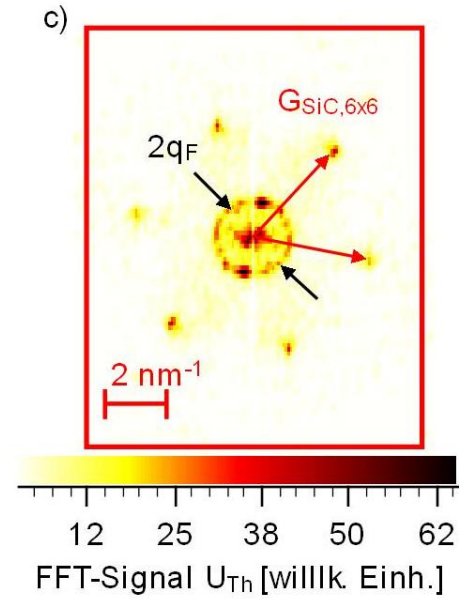

Abbildung 42: Signaturen der Intravalley-Streuung auf zweilagigem Graphen um den $\Gamma$-Punkt. (a) Topographie und (b) Thermospannungssignal $\left(I_{\mathrm{T}}=0.4 \mathrm{nA}, U_{\text {Bias }}=10 \mathrm{mV}\right.$, Datensatz aufgenommen in Zusammenarbeit mit [112]). (c) FFT-Darstellung der Thermospannungsdaten. In den Thermospannungs-daten überlagern sich die 6×6-Überstruktur mit langwelligen Modulationen der Wellenlänge von $\lambda_{\mathrm{F}} / 2=5,0(3) \mathrm{nm}$. In der FFTDarstellung sind diese durch einen isotropen Ring mit dem entsprechenden Wellenvektor bzw. Radius von $2\left|\mathbf{q}_{\mathbf{F}}\right|=1,24(5) \mathrm{nm}^{-1}$ sichtbar. Ursache der langwelligen Modulationen ist die sog. Intravalley-Streuung der Elektronen, bei der die Elektronen innerhalb eins Konus, welche um K bzw. K` liegen, vom Zustand $\mathbf{q}_{\mathbf{F}}$ in den Zustand $-\mathbf{q}_{\mathrm{F}}$ bei der Fermienergie gestreut werden.

In den Thermospannungsdaten sind deutlich langwellige Oszillationen mit einer Wellenlänge von $\lambda_{\mathrm{F}} / 2=5,0(3) \mathrm{nm}$ zu erkennen. Wie im oberen Absatz diskutiert, zeigt sich die Wellenlänge im FFT-Signal der Thermospannung durch einen isotropen Ring um $\Gamma$ mit dem Radius des doppelten Fermiwellenvektors, welcher experimentell bestimmt worden ist $\mathbf{z u}|2 \mathbf{q} \mathbf{F}|=1,24(5) \mathrm{nm}^{-1}$. Dieser Wert stimmt gut überein mit dem Wert von Mallet et al., 
die diesen über Messungen der differentiellen Leitfähigkeit um die Fermi-Energie bestimmt haben. Aus der Fermiwellenlänge kann die Elektronenkonzentration $n$ unter der vereinfachten Annahme einer linearen Dispersion mit Hilfe der Beziehung $n=k_{F}^{2} / \pi$ bestimmt werden zu $n \approx 1,3(2) \cdot 10^{13} \mathrm{~cm}^{-2}$. Die Elektronenkonzentration liegt damit in demselben Bereich wie die durch Photoemissionsdaten bestimmten Werte [19].

Ein weiteres auffälliges Merkmal in Abbildung 42 ist der unterschiedliche Thermospannungsoffset von ungefähr einem $\mathrm{mV}$ innerhalb des zweilagigen Graphens oberhalb und unterhalb des Wrinkles. Dies deutet darauf hin, dass es innerhalb von zweilagigem Graphen ebenso flächige Inhomogenitäten in der lokalen Zustandsdichte gibt, wie sie ebenso von Park et al. durch Thermospannungsmessungen beobachtet worden sind [113]. Nach S. Cho. et al. stellen Wrinkles auf epitaktischem Graphen verspannte Domänengrenzen zwischen verschiedenen AB-Stapelfolgen dar [89]. Geht man von dieser These aus, ist es denkbar, dass der Zwischenbereich in der Umgebung eines Wrinkles demnach keine AB-Stapelfolge besitzt, und somit andere elektronische Eigenschaften im Vergleich zu zweilagigem Graphen mit AB-Stapelfolge aufweisen.

Des Weiteren weisen die Thermospannungsoszillationen eine erhöhte Streuamplitude senkrecht zum topographischen Verlauf des Wrinkles auf, wie anhand des FFT-Signals zu erkennen ist. Dies deutet darauf hin, dass die Elektronen an diesen Defekten mit einer bestimmten Reflektivität gestreut werden. Da über B-Feld abhängige Ladungstransportmessungen auf epitaktisch gewachsenem Graphen auf $\mathrm{SiC}(0001)$ bei $4 \mathrm{~K}$ eine Phasenkohärenzlänge von $140 \mathrm{~nm}$ ermittelt worden ist [25], setzt sich jedoch die lokale Zustandsdichte an einem bestimmten Ort $\mathbf{r}$ aus der Superposition der Elektronenwellenfunktionen zusammen, deren Amplitude an dem betrachteten Ort von der in der Umgebung liegenden Defektkonfiguration und deren Streueigenschaften abhängig ist. Daher erhält man, wie in Abbildung 43 (b) gezeigt, ein komplexes Streumuster im Thermospannungssignal, dessen Analyse aufgrund der unzureichenden strukturellen Information nicht trivial ist. Abbildung 43 (c) zeigt eine entsprechend der Intravalley-Streuung erhöhte Intensität der Wellenvektoren $\mathbf{q F}$, dessen Richtungen senkrecht zu den ML-BL-Übergängen in der Topographie und den in Kapitel 3.5.3 identifizierten Wrinkles liegen. Ebenso ist ein Streubeitrag der Punktdefekte, welche in der Topographie in Abbildung 43 (a) erkennbar sind, denkbar. 
a)

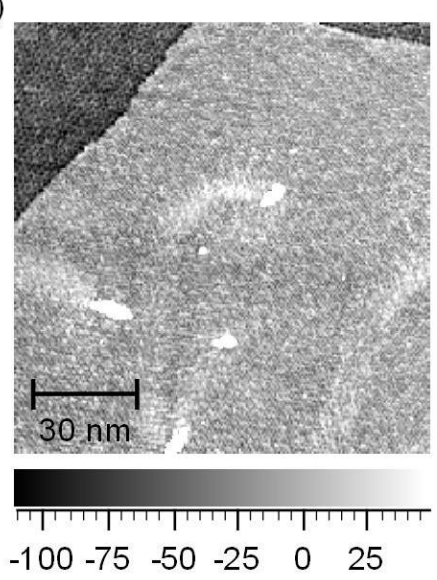

rel. Spitzenhöhe $[\mathrm{pm}]$ b)

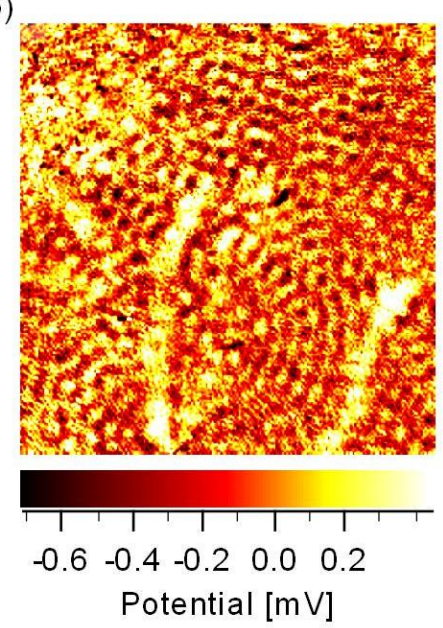

c)

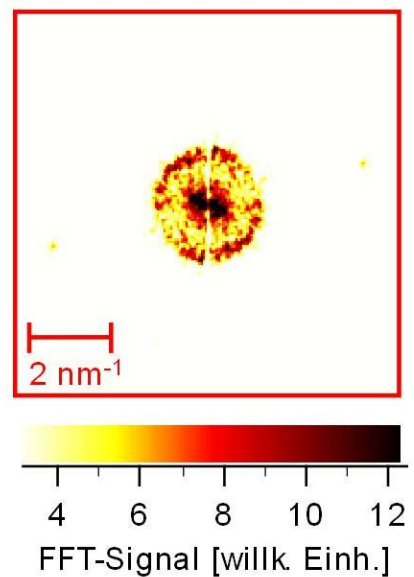

Abbildung 43: (a) Topographie von ein- und zweilagigem Graphen mit Wrinkles und Punktdefekten $\left(121 \times 118 \mathrm{~nm}^{2}, I_{\mathrm{T}}=0,1 \mathrm{nA}, U_{\text {Bias }}=20 \mathrm{mV}\right)$. (b) Potentiometrie-Signal mit komplexem Streumuster der Intravalley-Streuung durch Überlagerung der einzelnen Streubeiträge an den ML-BL-Übergängen, Wrinkles und Punktdefekten (Datensatz übernommen von [112]). (c) FFT-Ausschnitt mit Fermi-Ring, Streuvektoren in Richtung senkrecht zu den im Bild liegenden ML-BL-Übergängen und Wrinkles zeigen eine erhöhte Intensität, so dass davon ausgegangen werden kann, dass diese einen erheblichen Streubeitrag leisten.

Neben den bisher bekannten und hier auch in der Thermospannungsspektroskopie identifizierten elektronischen Signaturen um die Fermi-Energie, welche durch die Inter- und Intravalley-Streuung hervorgerufen werden, zeigt das Potentiometrie-Signal auch die 6×6Überstruktur, wie z.B. in Abbildung 42 (b) und (c) zu sehen ist. Bisher wurde angenommen, dass in zweilagigem Graphen diese, durch den Interface-Layer verursachte Überstruktur, nur aufgrund einer Höhenmodulation der zweiten Lage in STM-Topographien und Spektroskopien sichtbar ist, aber keinen wesentlichen elektronischen Beitrag bei der Fermi-Energie leisten [114]. Die Thermospannungsdaten zeigen jedoch, dass die Zwischenschicht ebenso in zweilagigem Graphen zu Änderungen in der lokalen Zustandsdichte führen. Es muss daher der Schluss gezogen werden, dass auch zweilagiges Graphen bei der FermiEnergie nicht vollständig von der Zwischenschicht entkoppelt ist. Da der Transport der Elektronen im linearen Transportregime hauptsächlich durch Zustände bei der Fermi-Energie getragen wird, müssen daher auch Streuprozesse berücksichtigt werden, welche durch die Störung der elektronischen Zustände des zweilagigen Graphens durch die Zwischenschicht hervorgerufen werden.

Dieses Kapitel zusammenfassend kann gesagt werden, dass es durch die Konstruktion des Rastertunnelmikroskops bei $6 \mathrm{~K}$ zu einer unterschiedlichen Proben- und Spitzentemperatur 
kommt und daher eine Thermospannung im Tunnelkontakt auftritt. Auf Graphen sind die mit der Potentiometrie bestimmten Thermospannungswerte in der Größenordnung von einigen $100 \mu \mathrm{V}$. Die Thermospannungsmessungen bieten die Möglichkeit, Streumechanismen direkt bei der Fermienergie zu analysieren. Dabei konnten die elastischen Streuprozesse der Inter- und Intravalley-Streuung identifiziert werden. Die Streusignaturen der Intravalley-Streuung deuten darauf hin, dass Elektronen sowohl an Übergängen von einund zweilagigem Graphen wie auch an auftretenden Wrinkles gestreut werden. Zusätzlich konnte für zweilagiges Graphen gezeigt werden, dass die Zwischenschicht eine Änderung der lokalen Zustandsdichte bei $E_{\mathrm{F}}$ hervorruft. 


\section{Ladungstransportmessungen von der atomaren bis zur mesoskopischen Skala auf epitaktischem Graphen}

\subsection{Untersuchung des Graphen-Metall-Kontaktwiderstandes auf semi- isolierendem $\mathrm{SiC}(0001)$}

Die elektronischen Eigenschaften von Graphen, wie die hohe Mobilität und die gleichzeitige Möglichkeit der Kontrolle der Ladungsträgerkonzentration über ein Gate machen es zu einem aussichtsreichen Kandidaten für zukünftige logische elektronische Bauelemente, die bisherige auf Halbleitern basierende Technologien ergänzt oder möglicherweise ersetzen. Neben den Transporteigenschaften der Graphenschicht, welche durch das darunterliegende Substrat sowie der Art und Anzahl der Defekte geprägt ist, spielt für die elektronischen Bauelemente ebenso das Metall-Graphen-Interface eine entscheidende Rolle, da eine Kontaktierung von Graphen für zukünftige elektronische Anwendungen unumgänglich ist. Für effiziente elektrische Bauelemente ist ein möglichst geringer Kontaktwiderstand zwischen den Metallelektroden und der Graphenschicht nötig [115]. Im Folgenden soll dargestellt werden, wie mit Hilfe der Raster-Kelvin-Mikroskopie (RKM) neben lokalen Variationen der Austrittsarbeit von Graphenoberflächen, wie schon in Kapitel 3.4 gezeigt worden ist, ebenso Variationen im elektrostatischen Potential auf Graphen und an Graphen-MetallKontakten untersucht werden können, wenn ein elektrischer Strom durch die Struktur fließt. Lokale Transportmessungen auf Graphen, die auf der Methode der Raster-KelvinMikroskopie beruhen, wurden bisher auf funktionalisierten Graphen-Flakes sowie vom Graphit-Kristall abgelösten Graphen-Schichten durchgeführt [116, 117].

Die hier vorgestellten Ergebnisse wurden unter dem Titel „Graphene-metal contact resistivity on semi-insulating $6 \mathrm{H}-\mathrm{SiC}(0001)$ measured with Kelvin probe force microscopy“ in Appl. Phys. Lett. 103, 051601 (2013) veröffentlicht [118]. Messungen des folgenden Kapitels sind in Zusammenarbeit mit F. Lüpke im Rahmen der betreuten Bachelorarbeit entstanden [119]. Der prinzipielle Versuchsaufbau ist schematisch in Abbildung 44 dargestellt. 


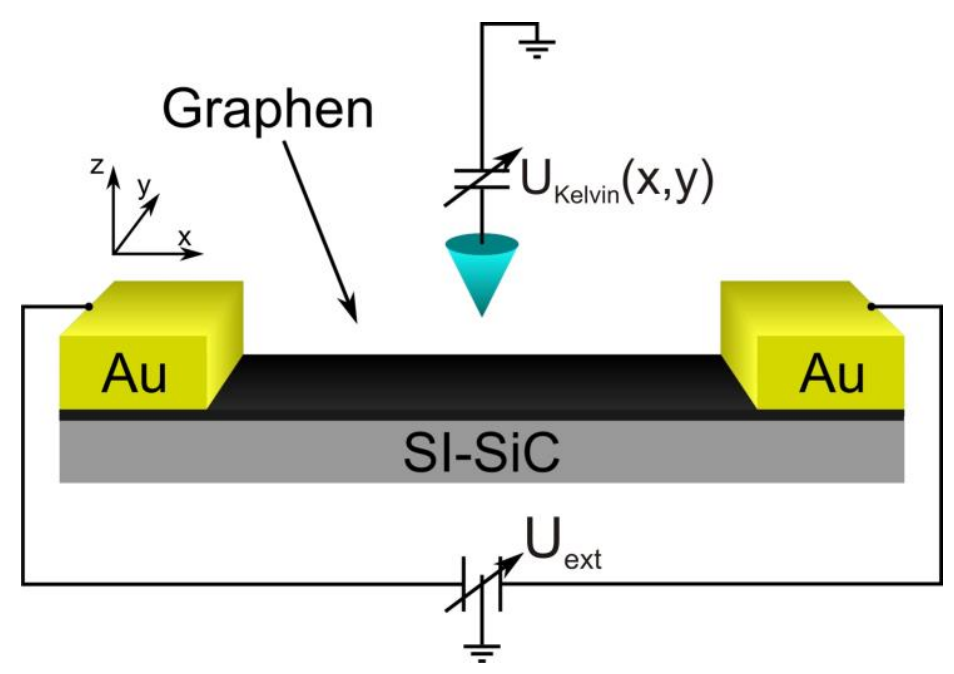

Abbildung 44: Schematische Darstellung des experimentellen Aufbaus. An die Goldkontakte wird eine Querspannung $U_{\text {ext }}$ angelegt, so dass ein elektrischer Strom durch die Graphenschicht (schwarz) fließt. Das lokale elektrostatische Potential der Probe wird durch den Abgleich mit dem elektrostatischen Potential der Spitze $U_{\text {Kelvin }}$ bestimmt.

Beim Transportexperiment wird zur Bestimmung lokaler Spannungsabfälle eine Querspannung $U_{\text {ext }}$ an die Probe angelegt. Die mit der Raster-Kelvin-Mikroskopie gemessene lokale Potentialdifferenz $U_{\text {Kelvin }}(x, y)$ setzt sich somit aus der Summe der lateral variierenden Kontaktpotentialdifferenz $U_{\mathrm{CPD}}(x, y)$ und der durch die angelegte Querspannung lokal variierenden elektrischen Potentialdifferenz $U_{\text {Trans }}(x, y)$ zusammen, so dass $U_{\text {Kelvin }}(x, y)=$ $U_{\mathrm{CPD}}(x, y)+U_{\text {Trans }}(x, y)$ gilt. Dabei wird angenommen, dass $U_{\mathrm{CPD}}(x, y)$ und $U_{\text {Trans }}(x, y)$ unabhängig voneinander sind [120]. Die Kontaktpotentialdifferenz ist dabei über die Differenz der Austrittsarbeit der Spitze $\phi_{\mathrm{S}}$ und Probe $\phi_{\mathrm{P}}$ über die Beziehung $U_{\mathrm{CPD}}=$ $-\left(\phi_{\mathrm{P}}(x, y)-\phi_{\mathrm{S}}\right) / e$ gegeben.

Unter der Voraussetzung, dass der Spannungsabfall über die Probe symmetrisch für entgegengesetzte Stromrichtungen ist $\left(U_{\mathrm{ext},+}=U_{\text {ext,-}}\right)$, kann somit $U_{\text {Trans }}(x, y)$ über die Beziehung

$$
U_{\text {Trans }}(x, y)=\frac{1}{2}\left(U_{\text {Kelvin }}^{+}(x, y)-U_{\text {Kelvin }}^{-}(x, y)\right)
$$

bestimmt werden. $U_{\text {Kelvin }}^{ \pm}(x, y)$ bezeichnet dabei das Messignal, welches für entgegen-gesetzte Polaritäten $U_{\text {ext, } \pm}$ bzw. Stromrichtungen aufgenommen worden ist. 

epitaktischem Graphen

Die Topographie- und Potentialmessungen wurden im Tapping-Modus bzw. frequenzmodulierten Kelvin-Mikroskopie-Modus an Atmosphäre bei Raumtemperatur durchgeführt. Im Gegensatz zu den verwendeten Proben bei den in dieser Arbeit gezeigten STMUntersuchungen sind die Ladungstransport-Messungen mit Hilfe der Raster-Kelvin-Methode auf einer Graphen-Probe, welche auf semi-isolierendem SiC(0001)-Substrat gewachsen wurde, untersucht worden. Die Präparation erfolgte durch indirektes resistives Heizen des semi-isolierenden SiC-Substrates durch ein darunterliegendes n-dotiertes Waferstück. Im Anschluss ist die Probe über $100 \mathrm{~nm}$ dicke Goldkontakte in Zweipunktgeometrie kontaktiert worden. Der Abstand der Kontakte betrug 1,54 mm, die Breite 2,48 mm. Der Widerstand der Probe war stromrichtungsunabhängig und lag bei $R_{\text {makro }}=810 \Omega$. Eine detaillierte Beschreibung der Präparation und Charakterisierung ist in der Bachelorarbeit von F. Lüpke zu finden [119].

Abbildung 45 zeigt eine $2 \times 2 \mu \mathrm{m}^{2}$ große Topographie und das simultan dazu aufgenommene Oberflächenpotential ohne angelegte Querspannung $\left(U_{\mathrm{ext}}=0\right)$. Im Gegensatz zu den Graphenproben, welche auf $\mathrm{n}$-dotiertem $\mathrm{SiC}(0001)$ durch direktes resistives Heizen präpariert worden sind, zeigt diese Graphenoberfläche kleinere Terrassen und eine Vielzahl von Wrinkles, welche erst ab zweilagigem Graphen auftreten [121]. Das Oberflächenpotential in Abbildung 45 (b) unterteilt sich in mehrere diskrete Werte, so dass zusammen mit der topographischen Beobachtung davon ausgegangen werden kann, dass die Graphenprobe mehrere Graphenlagen besitzt.

Abbildung 46 (a) zeigt einen $15 \times 5 \mu \mathrm{m}^{2}$ großen Topographieausschnitt, während eine Spannung von $U_{\text {ext }}= \pm 12 \mathrm{~V}$ an die Probe angelegt ist. Die Stromdichte durch die mehrlagige Graphenschicht beträgt dabei 5,97(14) A/m, so dass man über das ohmsche Gesetz und den gegebenen Abmessungen der Probe einen mittleren Flächenwiderstand des mehrlagigen Graphenfilms von $R_{\text {makro }}=1,31(6) \mathrm{k} \Omega / \square$ erhält. Abbildung 46 (b) bis (e) zeigt die zur Topographie aufgenommenen Oberflächenpotentiale sowie laterale, über den gesamten Probenbereich gemittelte Schnitte für beide Stromrichtungen. Die lineare Regression der Daten aus (d) und (e) zeigen einen nahezu symmetrischen Spannungsabfall mit einer gemittelten Steigung von 6,2(15) $10^{3} \mathrm{~V} / \mathrm{m}$, und man erhält mit der makroskopischen Stromdichte einen Flächenwiderstand von $R_{\text {mikro }}=1,04(4) \mathrm{k} \Omega / \square$. 
a)

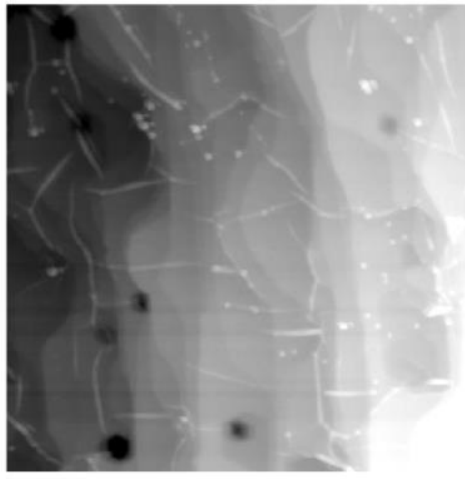

b)

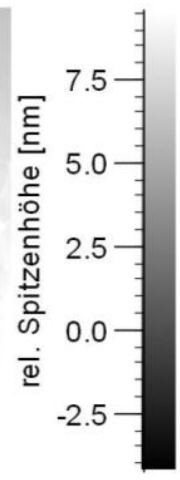

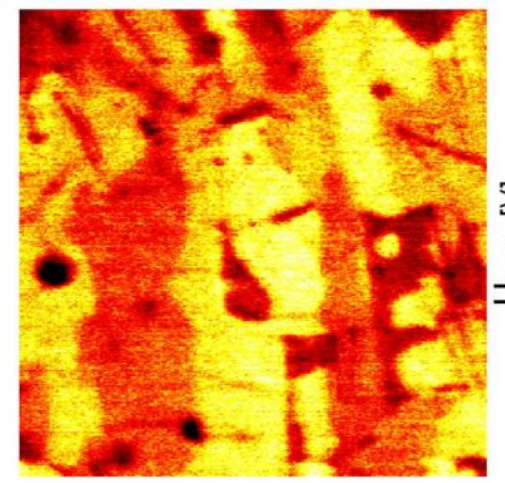

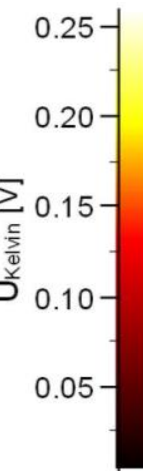

Abbildung 45: (a) $2 \times 2 \mu \mathrm{m}^{2}$ große AFM-Topographie sowie (b) simultan aufgenommenes Oberflächen-potential. Das Oberflächenpotential variiert räumlich und zeigt mehrere homogene Bereiche mit diskreten Werten. Anhand des Oberflächenpotentials kann davon ausgegangen werden, dass hier mehrlagiges Graphen vorliegt.

a)

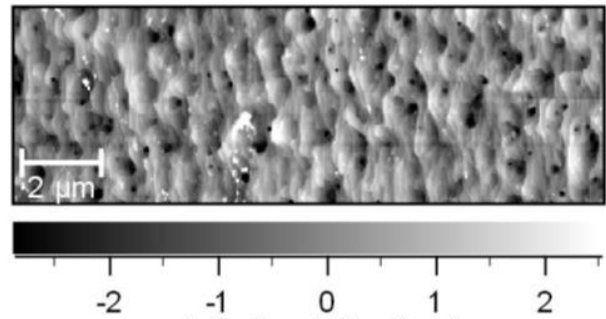

b)
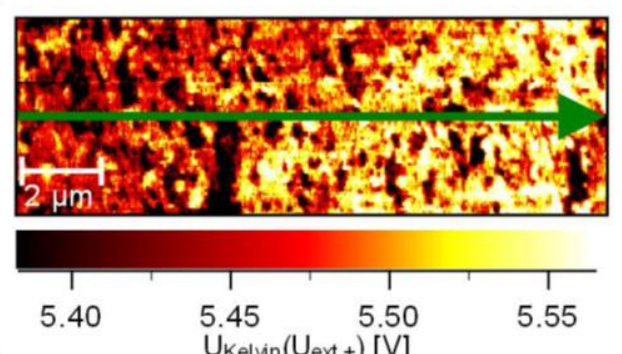

c)

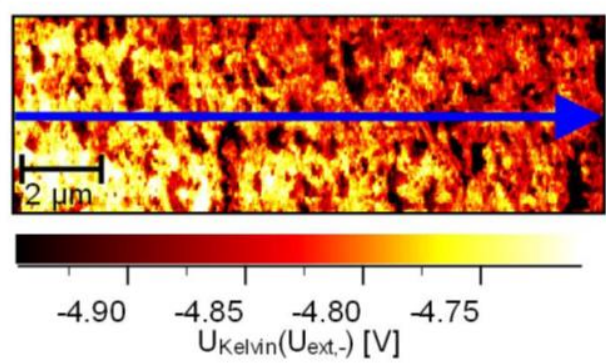

d)

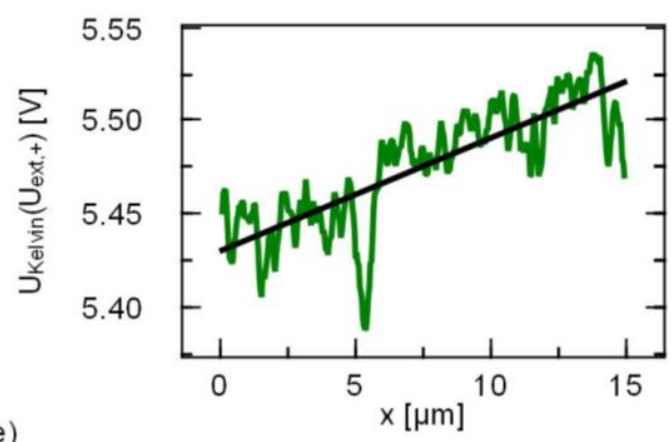

e)

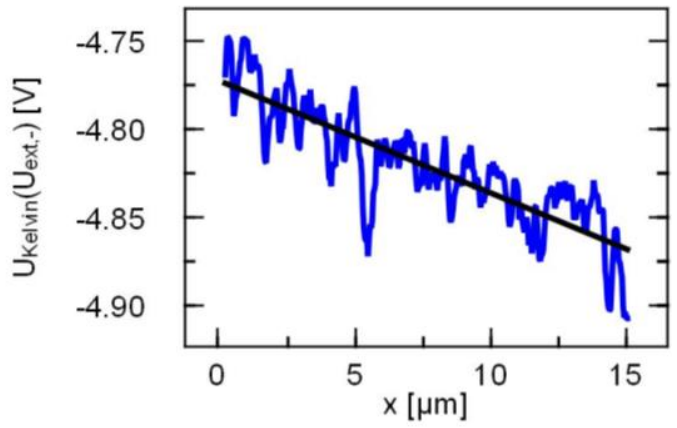

Abbildung 46: (a) $15 \times 5 \mu \mathrm{m}^{2}$ große Graphen-Topographie und (b), (c) aufgenommenes Oberflächenpotential bei einer lateralen Querspannung von $U_{\text {ext }}= \pm 12$ V. (d) und (e) Zugehörige über die gesamte Bildbreite gemittelte Schnitte aus (b) und (e) sowie linearer Fit. Das gemittelte elektrische Feld beträgt 6,06(22) $10^{3} \mathrm{~V} / \mathrm{m} \mathrm{bzw}$. $-6,33(20) \cdot 10^{3} \mathrm{~V} / \mathrm{m}$. 

epitaktischem Graphen

Um den Kontaktwiderstand zwischen den aufgedampften Goldkontakten und der Graphenschicht zu bestimmen, ist das Oberflächenpotential über einen der Goldkontakte bei einer Querspannung von $U_{\text {ext }}= \pm 8 \mathrm{~V}$ näher untersucht worden. Abbildung 47 (a) zeigt einen $20 \times 6 \mu \mathrm{m}^{2}$ großen Topographieausschnitt der Kontaktregion. Der Kontakt weist eine scharfe Kante zur Graphen-Schicht auf und hat eine Höhe von ca. 100 nm. Das Oberflächenpotential ist in Abbildung 47 (b) und (c) für entgegengesetzte Stromrichtungen $\left(U_{\text {ext }}= \pm 8 \mathrm{~V}\right.$ ) dargestellt. Die Graphenschicht zeigt im Mittel eine höhere Kontaktpotentialdifferenz als der aufgedampfte Gold-Kontakt und besitzt eine um 0,3(16) eV niedrigere Austrittsarbeit.
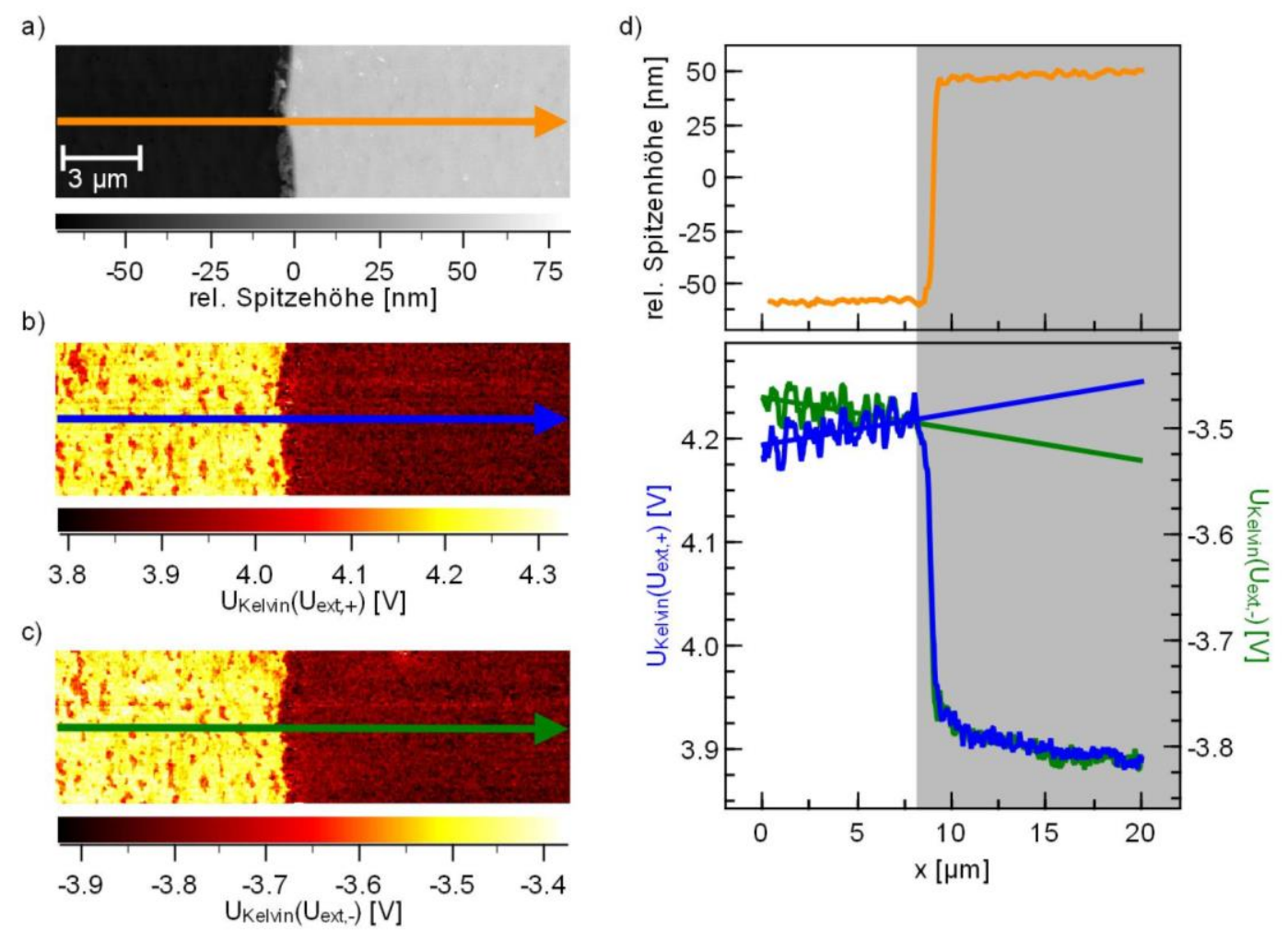

Abbildung 47: (a) 20×6 $\mu \mathrm{m}^{2}$ große Topographie am Goldkontakt und (b, c) dazugehöriges ortsaufgelöstes lokales Oberflächenpotential bei $U_{\text {ext }}= \pm 8$ V. (d) Lateral gemittelte Schnitte entlang der in (a) bis (c) durch Pfeile markierten Bereiche. Die laterale Position des Goldfilmes ist grau schraffiert.

Dieser gemittelte Wert stimmt gut mit RKM-Messungen von O. Kazakova et al. überein [76]. Wie auf den freien Graphenflächen ist auch hier ein Gradient im elektrostatischen Potential auf der Graphenfläche zu erkennen.

Dieser beträgt für beide Stromrichtungen im Mittel $\pm 3,00(24) \cdot 10^{3} \mathrm{~V} / \mathrm{m}$. Der daraus errechenbare Flächenwiderstand beträgt $0,75(7) \mathrm{k} \Omega / \square$ und liegt etwas unterhalb des zuvor auf 
der freien Fläche ermittelten Wertes. Beide ermittelten Werte zeigen, dass eine gute Übereinstimmung mit dem makroskopisch bestimmten Flächenwiderstand vorliegt und die Abweichung in der Größenordnung von \pm 30 \% liegt. Die Abweichung zwischen den makroskopisch und mikroskopisch bestimmten Flächenwiderständen können durch die Anzahl der lokalen Defekte wie Stufenkanten sowie einer inhomogenen Verteilung der GraphenLagenanzahl über die Probenoberfläche resultieren [122]. In Kapitel 5.2 bis 5.4 wird anhand von höchstauflösenden Rastertunnelpotentiometrie-Messungen der Einfluss von Defekten wie Stufenkanten auf den elektrischen Transport näher untersucht.

Änderungen der elektronischen Eigenschaften der Graphenschicht durch eine Diffusion von größeren Mengen Gold kann hier aus zwei Gründen ausgeschlossen werden. Zum einen deuten der stark lokalisierte Übergang im Oberflächenpotential an der Goldkante sowie der langreichweitige Verlauf des Oberflächenpotentials auf der Graphenschicht darauf hin, dass keine größere Menge Gold von den Goldkontakten in die Graphenschicht stattgefunden haben kann. Atomare Defekte können zwar nicht ausgeschlossen werden, ihr Einfluss kann jedoch als gering eingeschätzt werden, da diese nicht detektiert worden sind. Im Übrigen wurde die Probentemperatur während und nach der Deposition der Goldkontakte auf Raumtemperatur gehalten. Daraus kann geschlossen werden, dass bei der hier vorliegenden Raumtemperaturdeposition der Goldkontakte eine Golddiffusion im Bereich von einigen $\mu \mathrm{m}$ auszuschließen ist. Der ermittelte Flächenwiderstand liegt im Bereich bisheriger mit verschiedenen Methoden bestimmten Flächenwiderstand von $2,7 \mathrm{k} \Omega /$ auf funktionalisierten Graphen-Schichten [116], 0,3 k $\Omega / \square$ auf von Graphit-Kristall abgelösten Graphen-Schichten [123] und $0,2 \mathrm{k} \Omega /$ bis $0,8 \mathrm{k} \Omega / \square$ auf epitaktischem Graphen auf $\operatorname{SiC}(0001)[122,124]$.

Um den lokalen Spannungsabfall $U_{\text {Trans }}(x, y)$ über der Kontaktregion zu bestimmen, wurde nach Gleichung 5.1 die Differenz der für entgegengesetzte Stromrichtungen bestimmten Oberflächenpotentiale aus Abbildung 47 (b) und (c) bestimmt. Die in vertikaler Richtung gemittelten Werte sind in Abbildung 48 (a) dargestellt. Während auf dem Goldfilm aufgrund der wesentlich höheren Leitfähigkeit kein Gradient zu erkennen ist, zeigt der Bereich der Graphenschicht einen deutlichen lateralen Spannungsabfall. Der Übergangsbereich der Stufenkante ist grau unterlegt, wie in Abbildung $47 \mathrm{zu}$ sehen ist.

Um eine obere Grenze des Kontaktwiderstandes des Graphen-Gold-Übergangs anhand der in Abbildung 48 (a) gezeigten experimentellen Daten abzuschätzen, kann die Probe durch 

epitaktischem Graphen

ein zweidimensionales Widerstandsnetzwerk beschrieben werden, wie in Abbildung 48 (b) schematisch dargestellt ist. Dabei wird jedem Widerstand im Netzwerk entsprechend der Geometrie der Probe, der experimentell bestimmte Widerstand der Graphenschicht $R_{\text {mikro }}=0,75(7) \mathrm{k} \Omega / \square$, der spezifische Widerstand von Gold mit 22,14 $\mathrm{n} \Omega \cdot \mathrm{m}$ bei Raumtemperatur sowie der Kontaktwiderstand zugeordnet. Die Potentialwerte an jedem Knotenpunkt des Widerstandsnetzwerkes werden über das Knotenpotentialverfahren bestimmt. Als Randbedingungen wird der rechte Kontakt auf das experimentell bestimmte Potential und der linke Kontakt auf das Potential gesetzt, welches man durch Extrapolation des auf der Graphenschicht bestimmten Spannungsabfalles erhält.

Als Randbedingung dienen die am Kontakt bestimmten Werte des Flächenwiderstandes der Graphenschicht $R_{\text {mikro }}=0,75(7) \mathrm{k} \Omega / \square$, die makroskopisch bestimmte Stromdichte von $j_{\text {makro }}=3,98(14) \mathrm{A} / \mathrm{m}$ sowie die spezifische Leitfähigkeit von Gold bei Raumtemperatur von 22,14 $\mathrm{n} \Omega \cdot \mathrm{m}$. Für die Anpassung der in Abbildung 48 experimentell gezeigten Daten wurde als einziger freier Parameter der Simulation der Kontaktwiderstand des Gold-Graphen-Interfaces variiert.

Abbildung 48 (a) zeigt neben den experimentellen Daten die berechneten Potentialwerte der Graphenschicht für drei spezifische Kontaktwiderstände von $\rho_{\mathrm{c}}=5 \times 10^{-5} \mathrm{\Omega cm}^{2}$, $1 \times 10^{-5} \Omega \mathrm{cm}^{2}$ und $1 \times 10^{-6} \Omega \mathrm{cm}^{2}$. Der Spannungsabfall am Graphen-Gold-Interface nimmt bei höheren Kontaktwiderständen zu und führt in der Nähe der Graphen-Gold-Kante zu einem nahezu parallelen Verschieben der Potentialwerte auf der Graphenschicht. In der eingefügten Graphik in Abbildung 48 (a) ist der räumliche Verlauf der anhand des Widerstandsnetzwerkes berechneten Stromdichte entlang des Graphen-Gold-Interfaces für die drei Kontaktwiderstände dargestellt (Symbole). Die nummerisch bestimmten Werte des Stromdichteverlaufs am Gold-Graphen-Interface stimmen gut mit den analytischen Lösungen des sog. Transmission Line Modells (TLM) von Murrman und Widman [125] überein, welche zusätzlich in der eingefügten Graphik in Abbildung 48 (a) durch Linien dargestellt sind. Der Verlauf der Stromdichte zeigt, dass mit zunehmendem Abstand von der GoldGraphen-Kante die Stromdichte abnimmt bzw. direkt an der Kante am höchsten ist. 


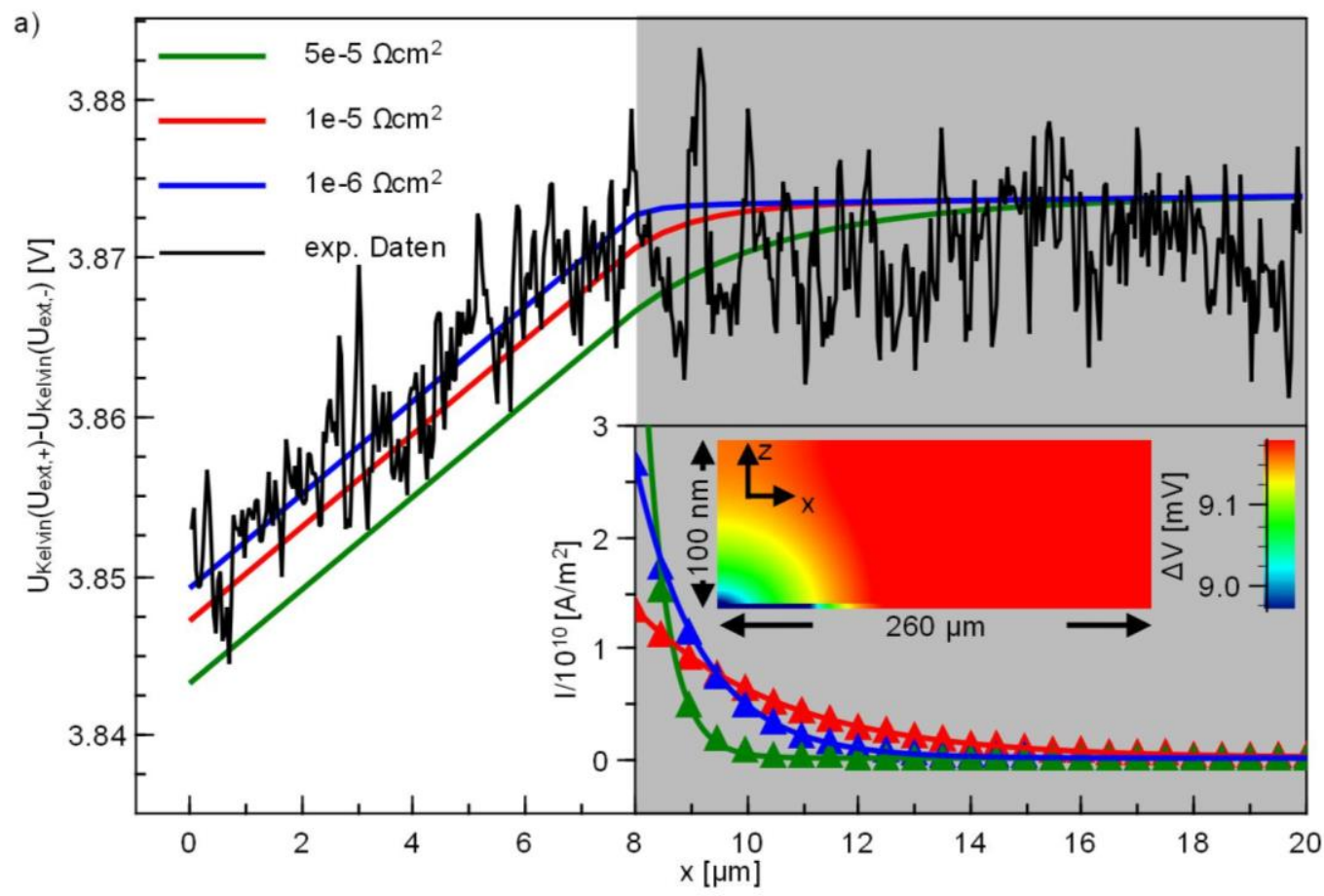

b)

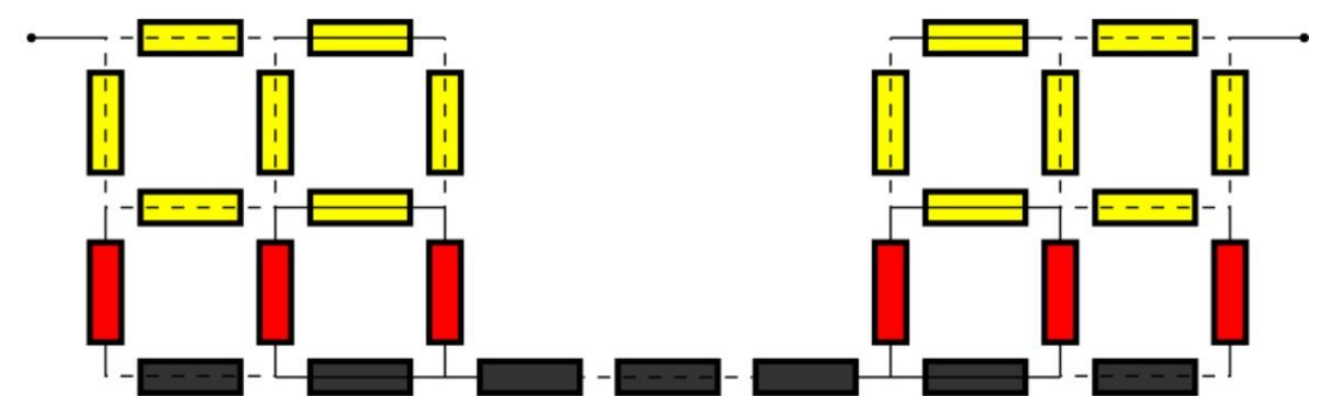

Abbildung 48: (a) Experimentell aus Abbildung 47 (b) und (c) bestimmter Potentialverlauf, über einen Graphen-Metallkontakt bei einer extern aufgeprägten Stromdichte von $j_{\text {makro }}=3,98(14) \mathrm{A} / \mathrm{m}$ sowie nummerisch berechneter Potentialverlauf der Graphenschicht für verschiedene Kontaktwiderstände von $\rho_{\mathrm{c}}=5 \times 10^{-5} \Omega \mathrm{cm}^{2}$, $1 \times 10^{-5} \Omega \mathrm{cm}^{2}$ und $1 \times 10^{-6} \Omega \mathrm{cm}^{2}$. Der grau schraffierte Bereich markiert die Position des Goldfilmes. In der eingefügten Grafik ist die entsprechende Stromdichteverteilung am Graphen-Gold-Interface dargestellt. Symbole markieren die nummerische Berechnung, Linien die analytische Lösung nach Murrman und Widman. Die zweidimensionale farbkodierte Graphik zeigt den Potentialverlauf über den gesamten Gold- Kontakt mit einer Länge von $260 \mu \mathrm{m}$ und Höhe von $100 \mathrm{~nm}$ bei $\rho_{\mathrm{c}}=1 \times 10^{-6} \Omega \mathrm{cm}^{2}$. (b) Schematische Darstellung des zur Simulation verwendeten Widerstandsnetzwerkes. Gelbe Widerstände stellen den Goldkontakt, schwarze die Graphenschicht und rote den Kontaktwiderstand des Graphen-Gold-Interfaces dar.

Dieser Effekt wird in der englischen Literatur auch als „current-crowding“ bezeichnet [125]. Das elektrostatische Potential über das Gold-Graphen-Interface verhält sich entsprechend der Stromdichte, wie in der eingefügten Grafik in Abbildung 48 (a) gezeigt ist. Der 
88 Ladungstransportmessungen von der atomaren bis zur mesoskopischen Skala auf epitaktischem Graphen

Potentialverlauf einer der Kontakte ist über die gesamte Kontaktlänge von $260 \mu \mathrm{m}$ und Kontaktfilmdicke von $100 \mathrm{~nm}$ in der Seitenansicht dargestellt. Durch den Vergleich der simulierten mit den experimentellen Daten kann eine obere Grenze für den Kontaktwiderstand zwischen der Graphenschicht und den Goldkontakten $\rho_{\mathrm{c}}=1 \times 10^{-6} \Omega \mathrm{cm}^{2}$ angegeben werden. Für höhere Kontaktwiderstände liegt die Differenz zwischen simulierten und experimentellen Potentialwerten außerhalb des Konfidenzintervalls der Messung von 1 $\sigma$. Die obere Grenze des ermittelten Kontaktwiderstandes liegt unterhalb des ermittelten Kontaktwiderstandes von $\rho_{\mathrm{c}}=5 \times 10^{-6} \Omega \mathrm{cm}^{2}$ für Nickel auf Graphen [115] und leicht oberhalb von $\rho_{\mathrm{c}}=6,3 \times 10^{-7} \Omega \mathrm{cm}^{2}$ für Gold auf funktionalisierten Graphenschichten [116] und liegt damit in der Größenordnung von bisherigen Messungen des Kontaktwiderstandes auf Graphen. 


\subsection{Rastertunnelpotentiometrie auf epitaktischem Graphen mit lateraler Quer- spannung}

In der Halbleiter-Industrie besteht ein besonderes Interesse an epitaktisch gewachsenem Graphen auf $\mathrm{SiC}$, da es großflächig auf einem ansonsten isolierendem Substrat gewachsen werden kann und sich aus der Halbleiter-Industrie zahlreiche Prozesschritte von bestehenden Produktionsverfahren übernehmen lassen. Im Allgemeinen liegt jedoch die Mobilität von epitaktischem Graphen auf $\mathrm{SiC}(0001)$ eine Größenordnung unter der Mobilität von auf Substraten wie hexagonalem Bornitrid abgelegtem oder freistehendem Graphen, welches nahe der Raumtemperatur eine Mobilität von über $2 \cdot 10^{5} \mathrm{~cm}^{2} \mathrm{~V}^{-1} \mathrm{~s}^{-1}$ erreichen können [126, 127]. Es stellt sich daher die Frage, welche morphologischen Eigenschaften der Oberfläche den elektrischen Transport beeinflussen. Beim epitaktischen Wachstum von Graphen kommt eine räumlich variierende Lagenanzahl sowie eine Vielzahl von verschiedenen Defekten, wie Stufen und Übergängen zwischen ein- und zweilagigem Graphen sowie Substratstufen vor. Dominante Streumechanismen zu identifizieren, ohne die genaue Kenntnis des Einflusses der einzelnen Defekttypen auszumachen, wird daher äußerst erschwert. Ein in den letzten Jahren verfolgter Ansatz ist unter anderem die Kombination von z.B. VierPunkt-Messungen mit an denselben Proben durchgeführten topographischen Untersuchungen über das Rasterkraft- oder Rasterelektronenmikroskop. Dabei konnte unter anderem eine Erhöhung der Leitfähigkeit und Mobilität mit abnehmender Stufendichte [128] und eine erhöhte Leitfähigkeit bei einem Stromfluss parallel zu Substratstufen im Vergleich zu einer senkrechten Orientierung beobachtet werden [129]. Im Folgenden wird der Einfluss von Defekten wie Substratstufen auf Längenskalen von $500 \times 500 \mathrm{~nm}^{2}$ bis auf Längenskalen unterhalb der Fermiwellenlänge auf ein- und zweilagigem Graphen mit der Methode der Raster-Tunnel-Potentiometrie erstmals bei $6 \mathrm{~K}$ untersucht. Der Ansatz lokale Transportuntersuchungen von epitaktischem Graphen auf $\mathrm{SiC}(0001)$ durchzuführen, die auf Basis der Raster-Tunnelpotentiometrie beruhen, wurde ebenso parallel zu dieser Arbeit von Clark et. al bei $81 \mathrm{~K}$ [130], Ji et. al. bei $77 \mathrm{~K}$ [122] und Wang et. al. [131] bei 17 K verfolgt. Diese werden am Ende des Kapitels mit den eigenen Resultaten diskutiert. 
a)

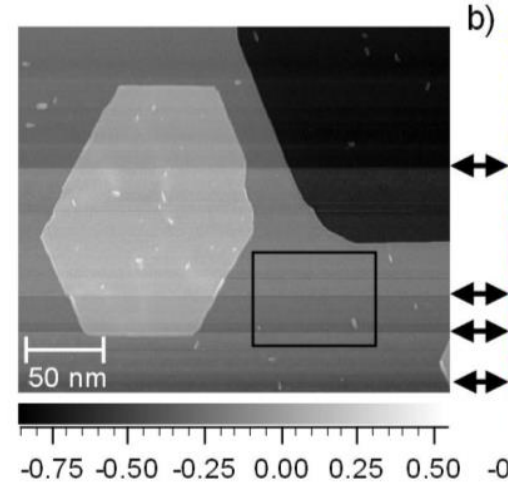

rel. Spitzenhöhe $[\mathrm{nm}]$ b)

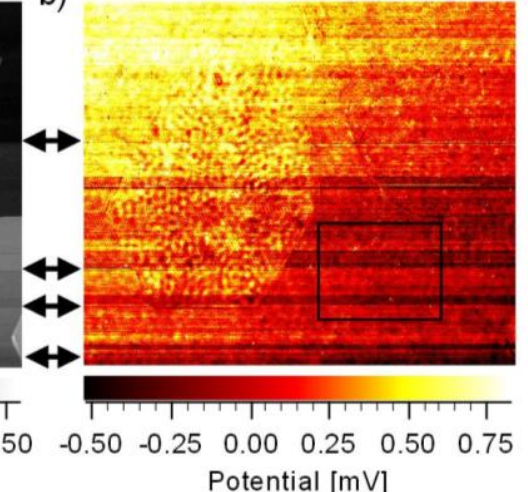

c)

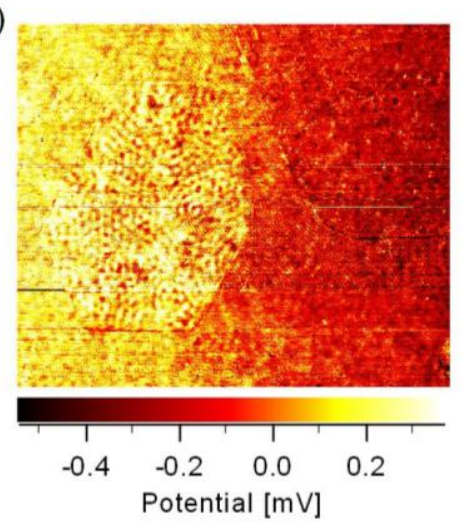

d)

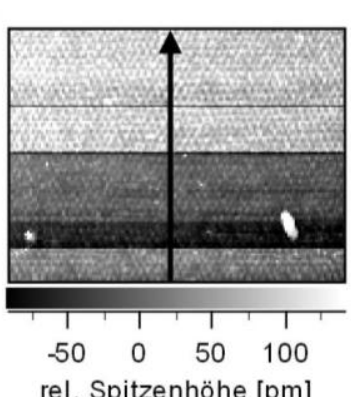

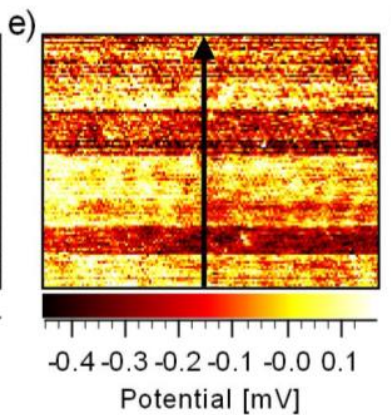

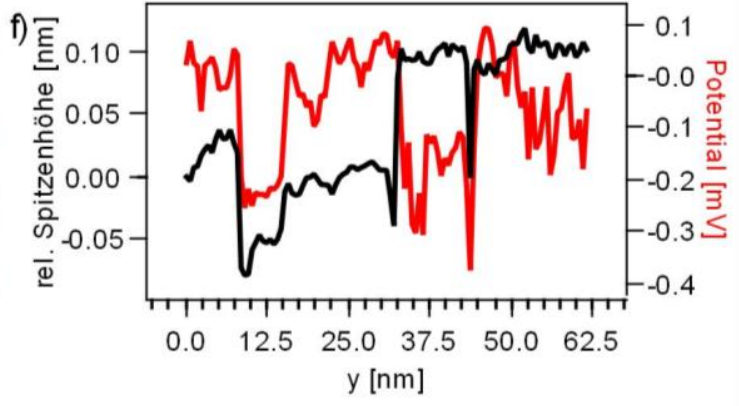

— rel. Spitzenhöhe $[\mathrm{nm}]$ — Potential $[\mathrm{mV}]$

Abbildung 49: (a) STM-Topographie der mit ein- und zweilagigem Graphen bedeckten SiC(0001)-Oberfläche ( $278 \times 236 \mathrm{~nm}^{2}, 510 \times 432$ Messpunkte, $\left.I_{\mathrm{T}}=235 \mathrm{pA}, U_{\text {Bias }}=50 \mathrm{mV}\right)$. (b) Unbearbeitete, simultan aufgenommene Potentiometrie-Daten bei einer mittleren makroskopischen Stromdichte von $j_{\text {makro }}=3,1$ (1) A/m durch die Probe. (c) Potentiometrie-Daten nach Subtraktion des Mittelwertes jeder Zeile entlang der schnellen (horizontalen) Rasterrichtung. (d), (e) Vergrößerte Ausschnitte der Topographie sowie Potentiometrie-Daten zeigen den Einfluss von Spitzenmodifikationen auf das Potentiometrie-Signal. (f) Gemittelte Werte der rel. Spitzenhöhe sowie des gemessenen Potentiometrie-Signals entlang der langsamen (vertikalen) Rasterrichtung. Abrupte, durch Spitzenmodifikationen hervorgerufene Änderungen von 20 bis 250 pm in der Spitzenhöhe führen zu Potentialsprüngen von bis zu einigen $100 \mu \mathrm{V}$.

Abbildung 49 zeigt einen Potentiometrie-Datensatz mit über 220000 Messpunkten. Die Messzeit des Bildausschnittes betrug ca. 12,5 Stunden und stellt gleichzeitig bei der gegebenen Auflösung die obere Grenze realisierbarer Messungen bei einer zur Verfügung stehenden Messzeit von ca. 15 Stunden dar. Messungen mit vergleichbarer energetischer wie auch räumlicher Auflösung bei $6 \mathrm{~K}$ sind in der Literatur zurzeit nicht bekannt. Die experimentelle Herausforderung besteht unter anderem darin, innerhalb der zur Verfügung stehenden Messzeit einen geeigneten Probenort zu finden, die STM-Spitze durch Spitzenpräparation in eine über die Messung stabile Konfiguration zu bringen und die optimalen Abbildungsparameter zu finden. 
Zur besseren Darstellung wurde in der Topographie in Abbildung 49 (a) die mittlere Ebene abgezogen. Das Potentiometrie-Signal ist in (b) unbearbeitet dargestellt. Die Topographie zeigt abrupte Sprünge entlang der langsamen (vertikalen) Rasterrichtung von $20 \mathrm{pm}$ bis 250 pm, die durch Spitzenmodifikationen innerhalb einer Rasterzeile hervorgerufen werden. Damit korreliert sind Sprünge im Potentiometrie-Signal in (b) ebenso entlang der langsamen Rasterrichtung. Schwarze Pfeile in Abbildung 49 (a) und (b) markieren einige Positionen. Zur besseren Vergleichbarkeit sind vergrößerte Bildausschnitte in (d) und (e) gezeigt. Aus gemittelten Schnitten entlang der langsamen Rasterrichtung, dargestellt in Abbildung 49 (f), wird deutlich, dass minimale Spitzenänderungen von 20 pm bis 250 pm, die ein nachregeln der rel. Spitzenhöhe zur Folge haben, Änderungen des Potentiometrie-Signals in der Größenordnung von einigen $100 \mu \mathrm{V}$ auslösen. Die Ursache hierfür ist die Änderung der elektronischen Eigenschaften der Spitze, wie der lokalen Austrittsarbeit oder aber auch der lokalen Zustandsdichte. Ändert sich eine der beiden genannten Größen während einer Messung, verändert sich ebenso nach Gleichung (4.1) der Absolutbeitrag der Thermospannung im Tunnelkontakt zum Potentiometriesignal. Daher ist es für die weitere Analyse der Potentiometriedaten in Bezug auf Ladungstransportuntersuchungen sinnvoll, den mittleren Potentialwert jeder Zeile entlang der schnellen (horizontalen) Rasterrichtung zu subtrahieren, wie in Abbildung (c) zu sehen ist. Durch diese Operation gehen strenggenommen gleichzeitig die Potential-Informationen entlang der langsamen Rasterrichtung verloren.

Zur weiteren Auswertung der Potentiometriedaten ist die Kenntnis der korrekten Abmessungen der Bilder sowie die absolute Orientierung der langsamen und schnellen Rasterrichtung in Bezug auf die Probe unerlässlich. Die gemessenen Proben wurden so in die Probenhalter eingebaut, dass bei angenommenem kartesischen Koordinatensystem die schnelle Rasterrichtung (x-Richtung) entlang der [11̄00]-Richtung verläuft und die langsame entlang der [11̄̄0]-Richtung. Eine mögliche Fehlorientierung der Proben im Probenhalter wird mit maximal $5^{\circ}$ abgeschätzt. Die Auswertung von atomar aufgelösten Bildern zeigt außerdem, dass die langsame und die schnelle Rasterrichtung nicht exakt senkrecht aufeinander stehen, sondern in einem Winkel von bis $\mathrm{zu} 84^{\circ}$ zueinander, so dass die mit dem Rastertunnelmikroskop aufgenommenen Datensätze eine leichte Scherung zeigen. Aufgrund des großen Kontaktabstandes wird davon ausgegangen, dass die makroskopische Stromrichtung der schnellen Rasterrichtung des STMs entspricht. Die durch die Scherung 

epitaktischem Graphen

und Fehlorientierung der Proben resultierende Winkelabweichung der schnellen Rasterrichtung von der makroskopischen Stromrichtung beträgt maximal $11^{\circ}$, wie anhand atomar aufgelöster Bilder ermittelt worden ist. Der daraus resultierende Fehler bezüglich der gemessenen Längen entlang der x-Achse ist somit unter insgesamt zwei Prozent und wird an dieser Stelle für die Auswertung der Transportmessungen vernachlässigt. Außerdem ist die x-Achse um max. $5 \%$ gestaucht und die y-Achse um max. $10 \%$ gestreckt. Da Potentialwerte aufgrund der obigen Argumentation nur sinnvoll entlang der x-Achse ausgewertet werden können, werden die in die Auswertung eingehenden Längenabmessungen um den systematischen gemittelten Fehler von 4 \% nach oben korrigiert.

Um aus gemessenen Potentialwerten den spezifischen Flächenwiderstand der topographischen Ausschnitte zu bestimmen, und diesen mit makroskopisch erhaltenen Werten aus Kapitel $3.4 \mathrm{zu}$ vergleichen, wird zunächst ein einfacher Ansatz des Ladungstransportes gewählt, nach dem sich gemäß des ohmschen Gesetzes $\boldsymbol{j}(\boldsymbol{r})=\sigma(\boldsymbol{r}) \cdot \boldsymbol{E}(\boldsymbol{r})$ die Stromdichte proportional zum lokalen elektrischen Feld ist. Das Potential an einem Ort $\phi(\boldsymbol{r})$ eines homogenen zweidimensionalen Leiters wird dann über die Laplace-Gleichung $-\nabla[\sigma$. $\nabla \phi(\mathbf{r})]=0$ bestimmt. Geht man davon aus, dass im betrachteten Probenbereich das Potential entlang der langsamen Rasterrichtung (y-Ache) invariant ist, verhält sich das Probenpotential linear entlang der schnellen Rasterrichtung (x-Achse). Damit gilt für das elektrische Feld $E_{x}=\left[\phi\left(x_{1}\right)-\phi\left(x_{2}\right)\right] / \Delta x$. Der spezifische Flächenwiderstand ist dann unter der Bedingung, dass die mikroskopische Stromdichte der makroskopischen entspricht gegeben über $\rho_{2 \mathrm{~d}}=\sigma_{2 \mathrm{~d}}^{-1}=E_{x} / j_{x, \text { makro }}$.

Abbildung 50 zeigt einen Potentiometriedatensatz, aufgenommen bei einem durch die Probe fließenden Strom von 5,2(1) mA und einer an den Kontakten angelegten Spannungsdifferenz von $U_{\text {ext }}=6,5 \mathrm{~V}$ bzw. $U_{\text {ext }}=-6,5 \mathrm{~V}$ für die Hin- und Rückspur. Die Topographien der Hin- und Rückspur sind in Abbildung 50 (a) und (b) dargestellt. Der Ausschnitt zeigt Bereiche mit ein- und zweilagigem Graphen sowie deren Übergänge des Typs I und II wie in Kapitel 3.2 beschrieben. Der Verlauf des lokalen elektrochemischen Potentials für entgegengesetzte Stromrichtungen ist in Abbildung 50 (c) und (d) gezeigt. Mit den entsprechenden Probenabmessungen (Kontaktabstand $l=4,2(1) \mathrm{mm}$ und Breite $b=1,8(1) \mathrm{mm}$ ) und den idealisierten Annahmen, dass der Strom homogen und nur in den oberen beiden Graphenlagen fließt, entspricht dies einer zweidimensionalen Stromdichte von 2,9(2) A/m 
bzw. $-2,9(2) \mathrm{A} / \mathrm{m}$ und einer makroskopischen Feldstärke von $\left|E_{x}\right|=\left|U_{\text {ext }} / l\right|=$ $(1550 \pm 37) \mathrm{V} / \mathrm{m}$.

a)

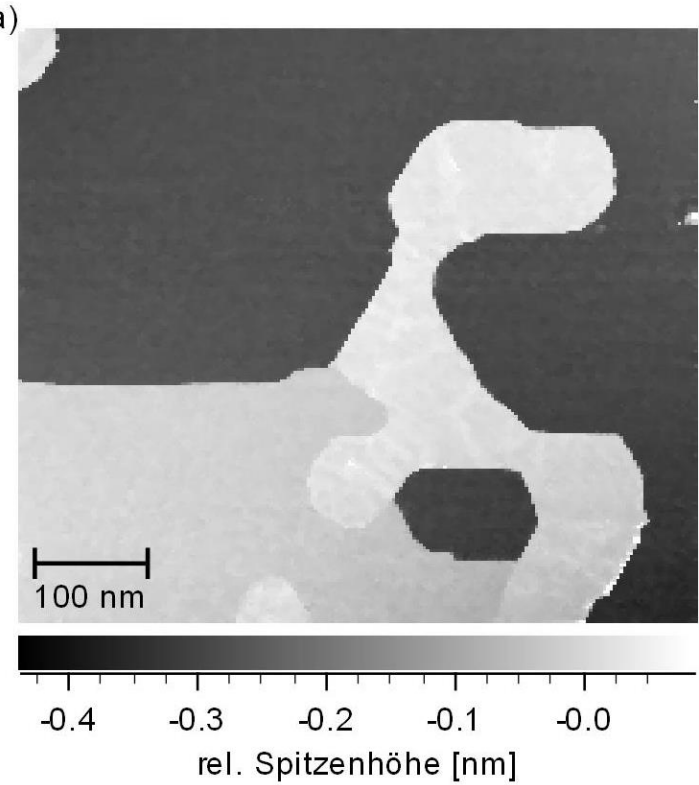

c)

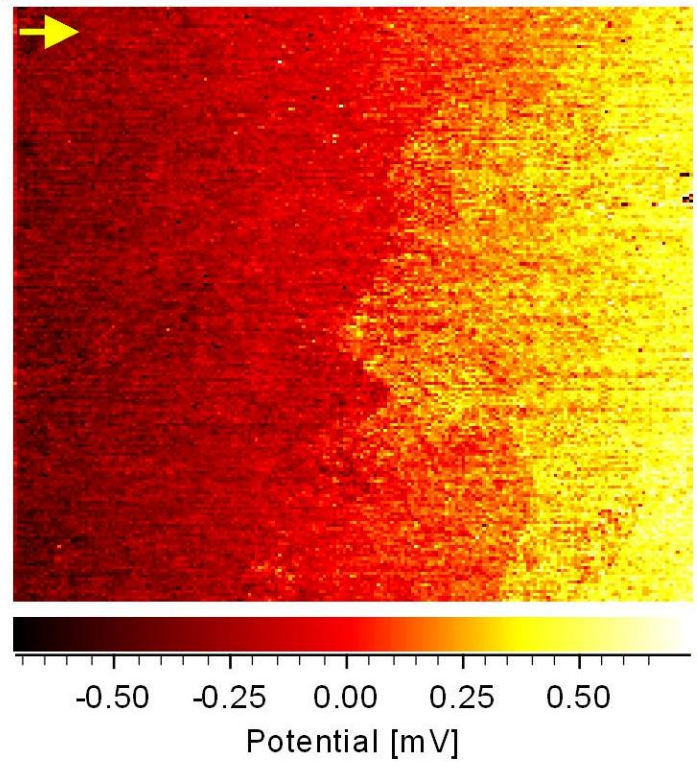

b)

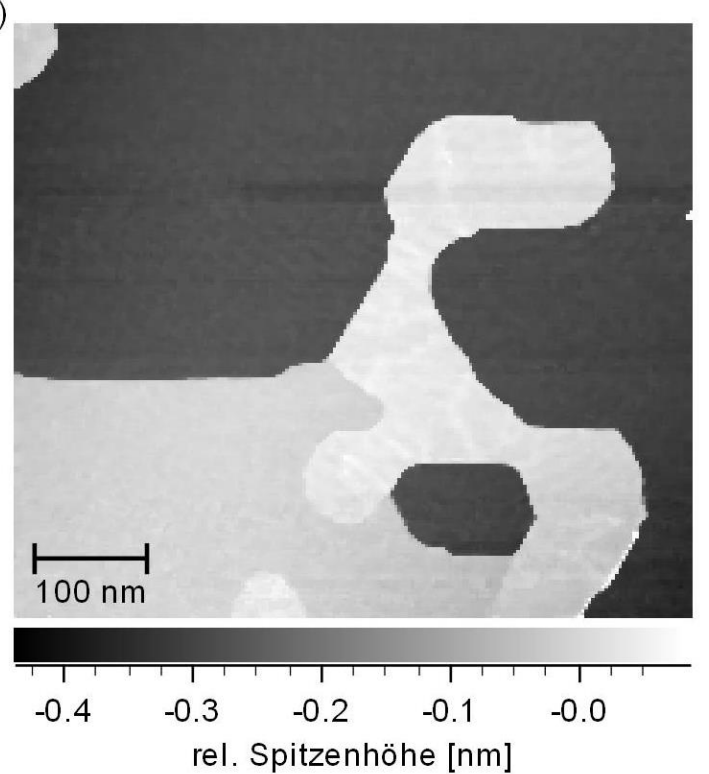

d)

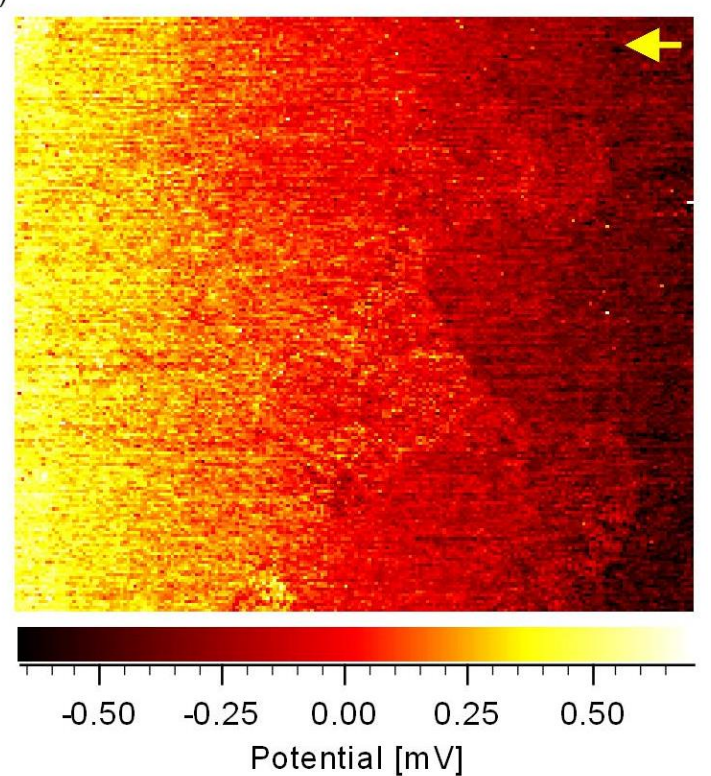

Abbildung 50: (a) Nachbearbeitete STM-Topographie $\left(624 \times 548 \mathrm{~nm}^{2}, 200 \times 200\right.$ Pixel, $U_{\text {Bias }}=30 \mathrm{mV}$, $I_{\mathrm{T}}=175 \mathrm{pA}$ ) mit ein- und zweilagigem epitaktischen Graphen entlang der Hinspur und (b) entlang der Rückspur sowie das jeweilig für entgegengesetzte Stromrichtungen aufgenommene lokale Probenpotential bei einer mittleren makroskopischen Stromdichte in horizontaler Richtung von 2,9(2) A/m in (c) bzw. -2,9(2) A/m in (d) und einem makroskopisch angelegtem elektrischen Feld von $\left|E_{x}\right|=(1550 \pm 37) \mathrm{V} / \mathrm{m}$. Gelbe Pfeile markieren die physikalische Stromrichtung. 
94 Ladungstransportmessungen von der atomaren bis zur mesoskopischen Skala auf epitaktischem Graphen

Die physikalische Stromrichtung ist mit gelben Pfeilen in den Abbildungen markiert. Die Fehler ergeben sich aus der Reproduzierbarkeit der makroskopischen Strommessung mit einer Genauigkeit von $2 \%$ und der Unsicherheit der Probenbreite.

Um den spezifischen Flächenwiderstand aus den Potentiometriedaten zu gewinnen, werden sichtbare lokale Variationen des elektrochemischen Potentials innerhalb des Bildausschnittes zunächst vernachlässigt und der Spannungsabfall über die Randbereiche der Abbildung 50 (c) und (d) bestimmt. Dazu werden die Potentialwerte aller Messpunkte entlang der langsamen Rasterrichtung des ersten und letzten Messpunktes der schnellen Rasterrichtung in einem Histogramm aufgetragen und der Mittelwert über einen Gauß-Fit ermittelt, wie in Abbildung 51 dargestellt ist. Somit erhält man für die Hin- und Rückspur einen Spannungsabfall von 858(10) $\mu \mathrm{V}$ bzw. -888(11) $\mu \mathrm{V}$ auf $611 \mathrm{~nm}$, ein gemitteltes elektrisches Feld von $\left|E_{x}\right|=1404(16) \mathrm{V} / \mathrm{m}$ bzw. $\left|E_{x}\right|=1453(19) \mathrm{V} / \mathrm{m}$, und damit einen spezifischen Flächenwiderstand von 484(34) bzw. 501(35) $\Omega / \square$. Diese Werte liegen in guter Übereinstimmung mit dem makroskopisch bestimmtem Flächenwiderstand derselben Probe von 537(33) $\Omega / \square$.

a)

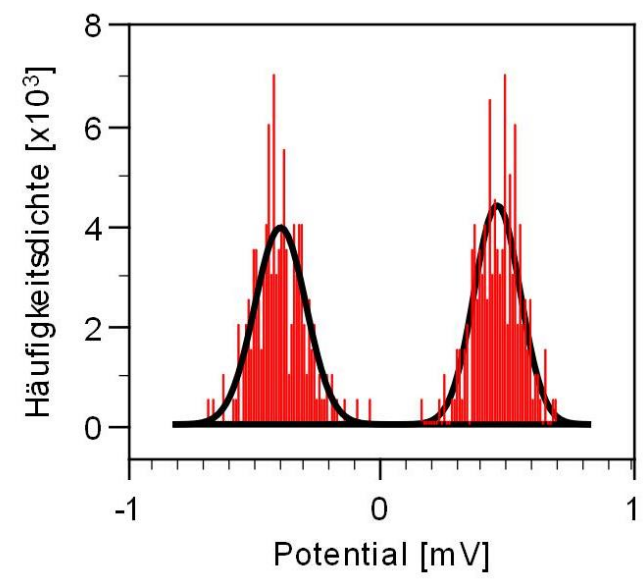

b)

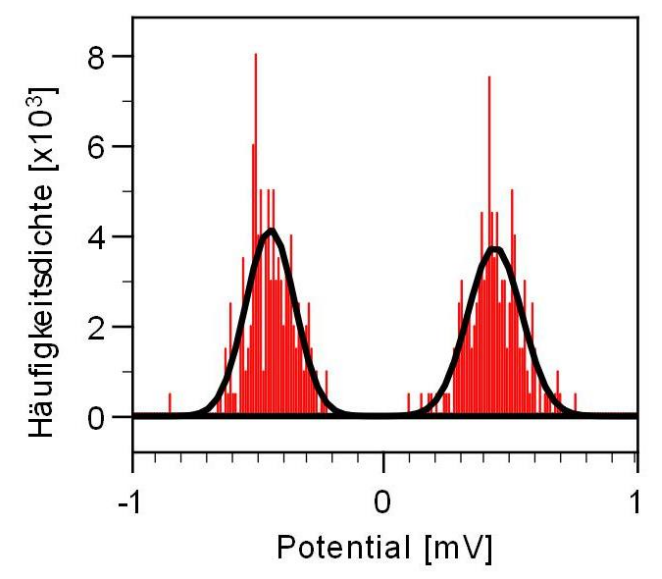

Abbildung 51: Zur Bestimmung des Spannungsabfalles: Histogramme der Randbereiche der Potentiometriedaten aus Abbildung 50, entlang der langsamen Rasterrichtung für (a) Hinspur und (b) Rückspur.

Dies bedeutet, dass im Rahmen der Messtoleranz der Spannungsabfall auf diesen Längenskalen zum einen von der Stromrichtung unabhängig ist und zum anderen, wie die sehr gute 
Übereinstimmung mit den makroskopisch angelegten elektrischen Feldern zeigt, die mesoskopisch gemittelte Stromdichte innerhalb diese Bildausschnittes der makroskopischen Stromdichte entspricht.

Ebenso ist es gelungen, Potentialmessungen für verschiedene Stromdichten im selben Topographiebereich durchzuführen. Abbildung 52 (a) zeigt eine $500 \times 500 \mathrm{~nm}^{2}$ große Topographie und in (b) das dazugehörige lokale elektrochemische Potential für vier makroskopische Querstromdichten von $j_{x}= \pm 3,8(3) \mathrm{A} / \mathrm{m}$ und $j_{x}= \pm 1,9(1) \mathrm{A} / \mathrm{m}$. Trägt man das zugehörige mittlere elektrische Feld gegen die Stromdichte auf erhält man das nach dem ohmschen Gesetz erwartete lineare Verhalten.

a)

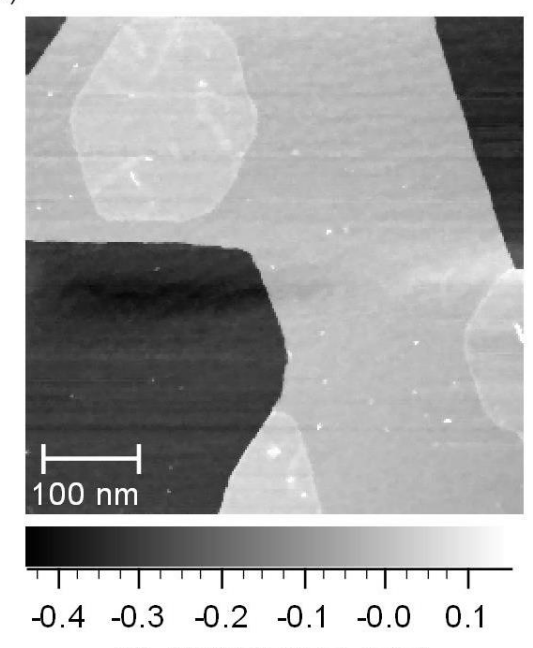

rel. Spitzenhöhe $[\mathrm{nm}]$ b)
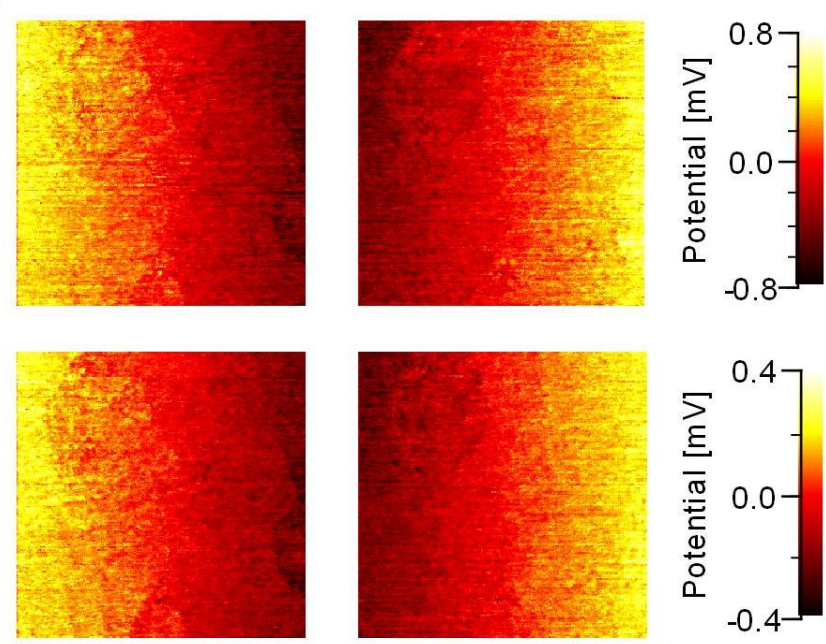

c)

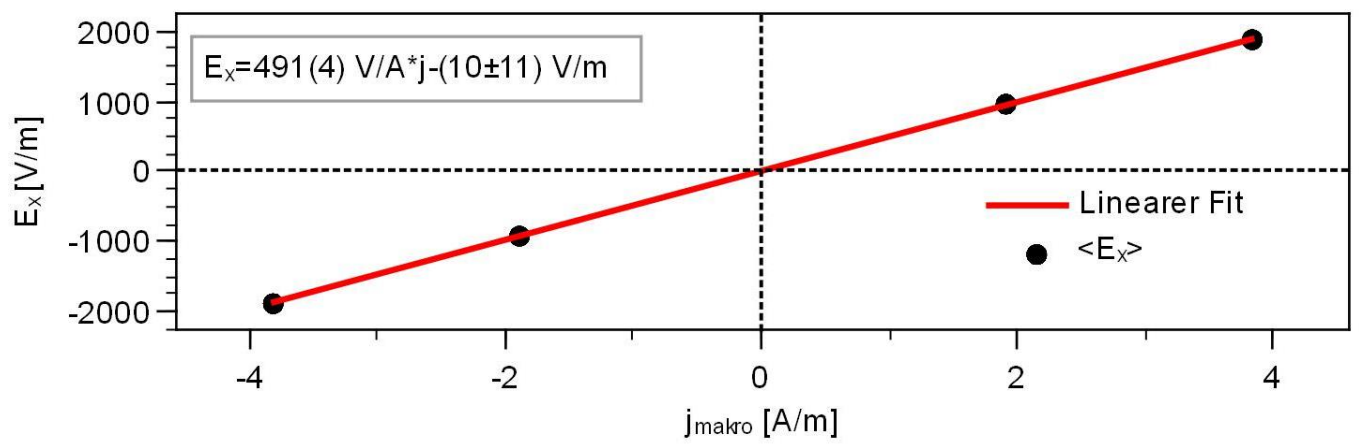

Abbildung 52: (a) 500 $\times 500 \mathrm{~nm}^{2}$ Topographie (256 $\times 256$ Pixel, $\left.I_{\mathrm{T}}=0,15 \mathrm{nA}, U_{\text {Bias }}=-0,03 \mathrm{~V}\right)$ und (b) dazu aufgenommenes lokales elektrochemisches Potential für vier makroskopische Stromdichten $j_{\text {makro. }}$ (b) Lineare Abhängigkeit des gemittelten elektrischen Feldes in Abhängigkeit von der makroskopisch angelegten Stromdichte entlang der langsamen Rasterrichtung. 
Der aus diesem Bildbereich ermittelte spezifische Flächenwiderstand beträgt 491(4) $\Omega / \square$ und weicht auch in diesem Bildausschnitt weniger als $10 \%$ vom makroskopischen Wert ab. Daher kann angenommen werden, dass innerhalb einer Bildgröße von ungefähr $500 \times 500 \mathrm{~nm}^{2}$ alle relevanten Streumechanismen enthalten sind und die mittlere mikroskopische Stromdichte nur kleine Abweichungen von der makroskopischen hat.

Um den Verlauf von $\mu_{\mathrm{ec}, \mathrm{STP}}$ auf kleineren Längenskalen zu untersuchen, ist es zunächst notwendig, das Verhalten der in Kapitel 4.2 beschriebenen Thermospannung während des Transportexperiments zu analysieren. Diese äußert sich als zusätzliche Spannungsquelle im STM und muss für die Auswertung der Transportdaten, wie im nächsten Kapitel gezeigt wird, mit einbezogen werden.

\subsubsection{Thermospannungsanteil an Ladungstransportmessungen mit der Raster- tunnelpotentiometrie sowie Grenzen der Auflösung}

Wie in Kapitel 4 diskutiert, tritt aufgrund des Temperaturunterschiedes zwischen Spitze und Probe eine Thermospannung im Tunnelkontakt auf, welche mit Hilfe der Rastertunnelpotentiometrie bestimmt werden kann. Die lateral variierende Thermospannung kommt dabei durch Änderungen in der lokalen Zustandsdichte zustande. Nach der in Kapitel 2.1.2 beschriebenen Theorie sollte sich im Fall von diffusivem Ladungstransport, die Spitzenspannung $U_{\mathrm{S}}$, die nötig ist, um den Tunnelstrom an einem Ort zu kompensieren, aus zwei Beiträgen zusammensetzen: der Thermospannung $U_{\text {Th }}$ und dem durch den lateralen Transport hervorgerufenen lokal variierenden Probenpotential $U_{\text {Trans }}$. Unter der Voraussetzung eines stromrichtungsunabhängigen Transportes invertiert sich für entgegengesetzte Stromrichtungen \pm einfach das Vorzeichen des lokalen Probenpotentials $\pm U_{\text {Trans }}$ im Gegensatz zum Vorzeichen der Thermospannung. Bildet man nun den Mittelwert der gemessenen Potentiale für entgegengesetzte Stromrichtungen, also $\left(U_{\mathrm{S},+}+U_{\mathrm{S},-}\right) / 2=$ $\left(U_{\mathrm{Trans}}+U_{\mathrm{Th}}\right) / 2+\left(-U_{\mathrm{Trans}}+U_{\mathrm{Th}} / 2\right)$, erhält man den reinen Thermospannungsanteil. Bildet man die Differenz, $\left(U_{\mathrm{S},+}-U_{\mathrm{S},-}\right) / 2$, erhält man den durch das Transportfeld resultierenden Spannungsabfall $U_{\text {Trans }}$. Um diese Hypothese zu überprüfen, wurden im Rahmen einer betreuten Masterarbeit von P. Willke [122] an ein und demselben Probenort zeilenweise zunächst $U_{\mathrm{Th}}$ und im Anschluss für entgegengesetzte makroskopische Stromrichtungen das Potentiometrie-Signal $U_{\mathrm{S}, \pm}$ bestimmt. Abbildung 53 (a) zeigt eine $375 \times 112 \mathrm{~nm}^{2}$ 
große Topographie mit einer ML-ML Stufe und einer zweilagigen Graphen-Terrasse auf der oberen Terrasse der einlagigen Graphenschicht. Abbildung 53 (b) zeigt das gemessene Thermospannungssignal ohne das lateral angelegte Transportfeld. Es wird ersichtlich, dass ein- und zweilagiges Graphen neben den in Kapitel 4.2 diskutierten unterschiedlichen Mo-

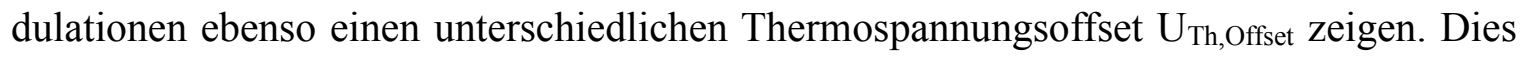
bedeutet nach der bisherigen Theorie der Thermospannung, dass ein- und zweilagiges Graphen unterschiedliche elektronische Eigenschaften bei der Fermi-Energie besitzen. In dem hier gezeigtem Beispiel beträgt die Differenz der über ein- und zweilagigem Graphen gemittelten Thermospannungswerte $U_{\mathrm{Th}, \mathrm{ML}}-U_{\mathrm{Th}, \mathrm{BL}} \approx 270 \mu \mathrm{V}$.

a)

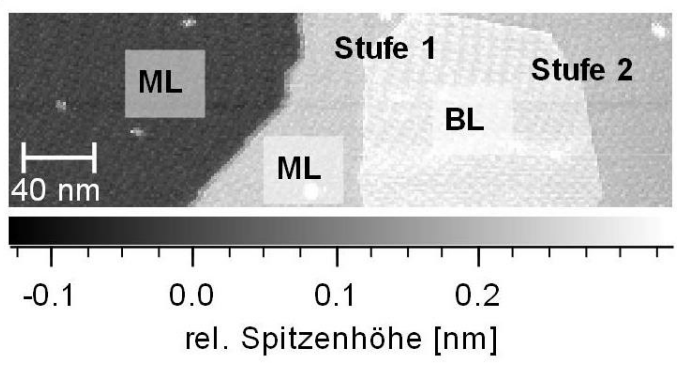

c)

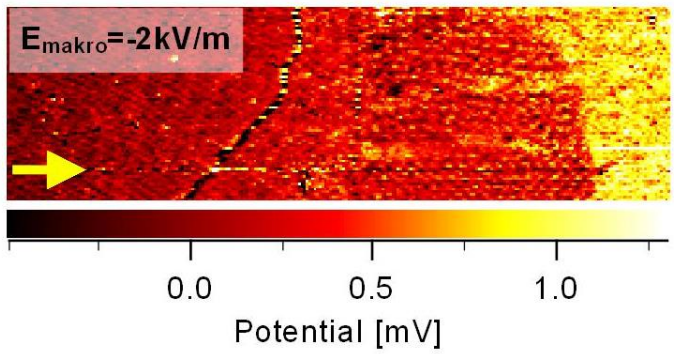

e)

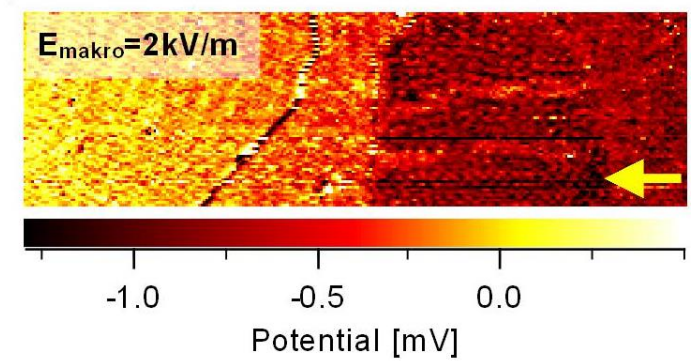

b)

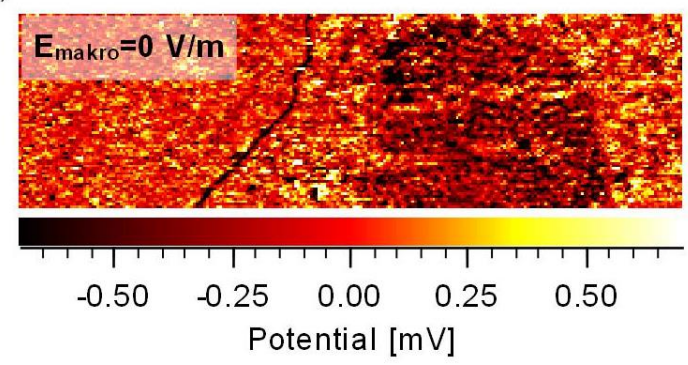

d)

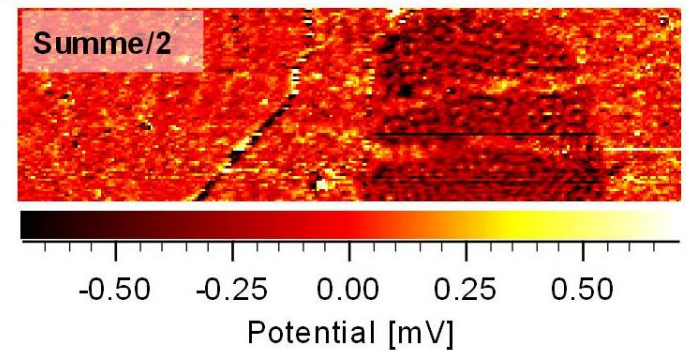

f)

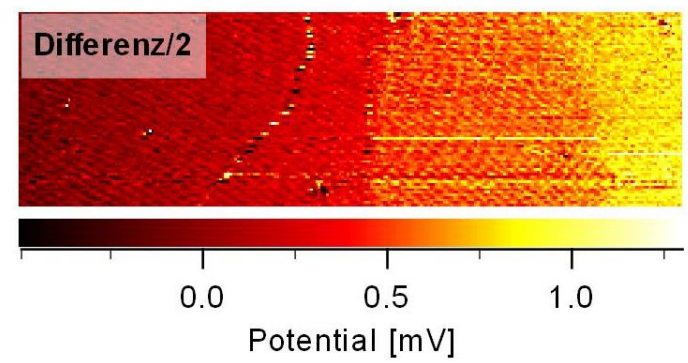

Abbildung 53: Experimentelle Evaluierung zum additiven Verhalten von $U_{\text {Th }}$ und $U_{\text {Trans }}$ zum PotentiometrieSignal (Datensatz übernommen von [112]).

In Abbildung 53 (c) und (e) sind die Potentialwerte für entgegengesetzte Stromrichtungen dargestellt. Auch hier ist ein scheinbar stromrichtungsabhängiger Spannungsabfall an den 

epitaktischem Graphen

Stufen 1 und 2 (vgl. Abbildung 53 (a)) erkennbar. Die Ursache hierfür ist jedoch nicht eine z.B. stromrichtungsabhängige Transmission der Stufenkanten, sondern der von der Stromrichtung unabhängige Beitrag der Thermospannung zu dem Potentiometrie-Signal. Bildet man den Mittelwert der Potentialwerte der entgegengesetzten Stromrichtungen aus Abbildung 53 (c) und (e), erhält man gerade den Thermospannungsbeitrag, wie in Abbildung 53 (d) dargestellt. Ein direkter Vergleich mit Abbildung 53 (b) zeigt eine sehr gute qualitative Übereinstimmung. Umgekehrt kann aus der Differenz der Potentialwerte beider entgegengesetzter Stromrichtungen der Thermospannungsanteil minimiert werden, wie es in Abbildung 53 (f) demonstriert ist.

Die Eliminierung der Thermospannung bietet den Vorteil, Spannungsabfälle zum einen genauer lokalisieren und zum anderen den tatsächlichen durch einen lateralen Strom hervorgerufenen Spannungsabfall an den ML-BL-Übergängen zu bestimmen. Am folgenden Datensatz soll der Einfluss der Spitze auf die gemessenen Beiträge der Thermospannung von ein- und zweilagigem Graphen näher untersucht werden.

Abbildung 54 zeigt ein weiteres Beispiel des Einflusses der Thermospannung auf die Ladungstransportmessungen. Die Rohdaten ohne weitere Bearbeitung sind in Abbildung 54 (a), (c) und (e) dargestellt. In der Topographie (Abbildung 54 (a), 274×124 $\mathrm{nm}^{2}$ ) ist eine zweilagige Graphen-Terrasse umgeben von einlagigem Graphen zu sehen. Durch Spitzenmodifikationen ändert sich die relative Spitzenhöhe an einigen Orten abrupt von einer Zeile zur nächsten von einigen pm bis hin zu in diesem Fall 250 pm, wie es durch den schwarzen Pfeil an einem Beispiel markiert ist. Solche Änderungen rufen in vielen Fällen ebenso eine Änderung der elektronischen Eigenschaften der Spitze hervor, und haben einen großen Einfluss auf die Potentiometriedaten. In der Regel werden zur besseren Darstellung der Topographien solche Höhenänderungen durch entsprechende graphische Bearbeitung der Daten durch z.B. den Abzug des mittleren Offsets je Zeile beseitigt (vgl. Abbildung 54 (b)). Abbildung 54 (c) und (e) zeigen die Potentialdaten für entgegengesetzte makroskopische Stromrichtungen $\left(j_{x}= \pm 3,2(2) \mathrm{A} / \mathrm{m}\right)$. Die aus den Potentialdaten (c) und (e) extrahierte Thermospannung ist in Abbildung 54 (d) dargestellt. Es zeigt sich, dass der Absolutbetrag der Thermospannung für ein- und zweilagiges Graphen $U_{\mathrm{Th}, \mathrm{ML} / \mathrm{BL}}$ in den Messungen nicht konstant ist und die Änderungen mit den in der Topographie beobachteten Spitzenmodifikationen korreliert sind. 


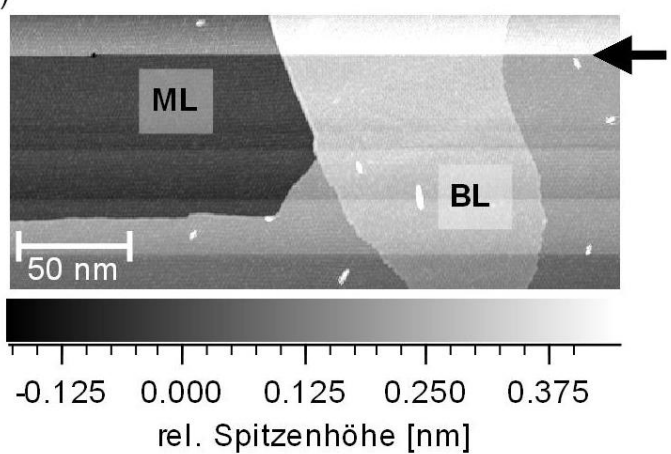

c)

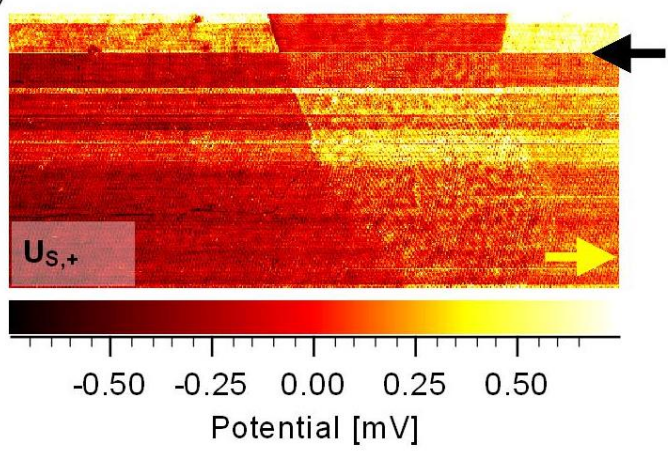

e)

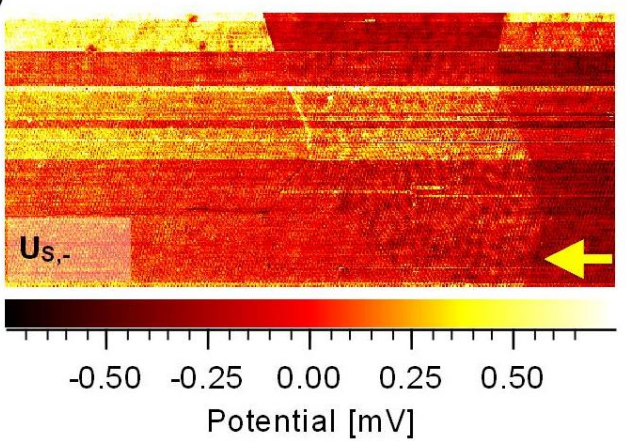

b)

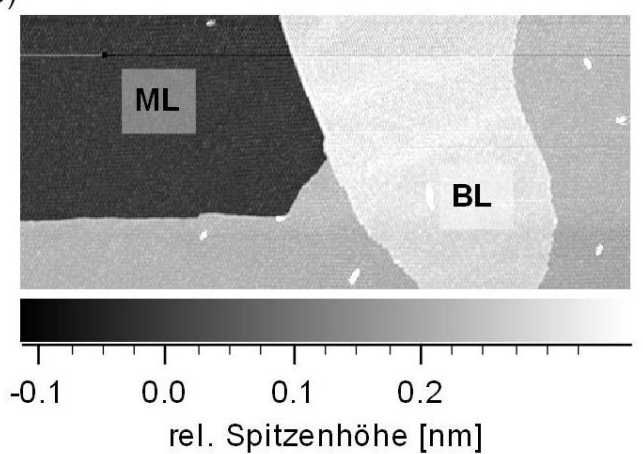

d)

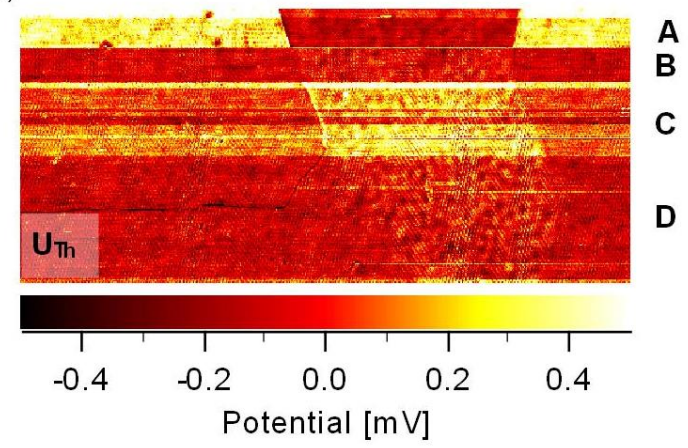

f)

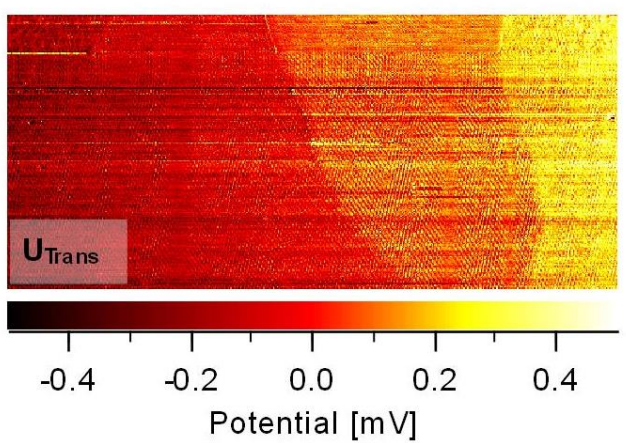

Abbildung 54: Einfluss und Eliminierung des Thermospannungsanteils $U_{\mathrm{Th}}$ vom Potentiometrie-Signal $U_{\mathrm{S}}$. (a) $274 \times 124 \mathrm{~nm}^{2}$ große Topographie einer von einlagigem Graphen umschlossenen zweilagigen Graphenterrasse vor und (b) nach Abzug des mittleren Offsets je Zeile $\left(I_{\mathrm{T}}=0,5 \mathrm{nA}, U_{\text {Bias }}=-40 \mathrm{mV}, 502 \times 228\right.$ Pixel). Die Topographie ist durch kleine Spitzenmodifikationen geprägt. (c) und (e) Rohdaten des Potentiometrie-Messsignals $U_{\mathrm{S},+}$ bzw. $U_{\mathrm{S},-}$ für entgegengesetzte Stromrichtungen $\left(j_{x}= \pm 3.2(2) \mathrm{A} / \mathrm{m}\right)$. (d) Differenz von (c) und (e) liefert den Thermospannungsanteil von $\mathrm{U}_{\mathrm{S}, \pm}$. Dieser zeigt unterschiedliche Thermospannungsdifferenzen zwischen ein- und zweilagigem Graphen zwischen $\Delta U_{\mathrm{Th} \text {,Offset }} \approx-250 \mu \mathrm{V}$ bis $\Delta U_{\mathrm{Th} \text {,Offset }} \approx 320 \mu \mathrm{V}$ abhängig von der aktuellen Spitzenkonfiguration. (f) Thermospannungsbereinigtes Potentiometrie-Signal $U_{\text {Trans }}$.

Wäre die Differenz der Thermospannungsbeiträge wiederum konstant, könnte man vermuten, dass in den Messungen das durch die Querspannung hervorgerufene lokale Potential am betrachteten Ort aufgrund von z.B. Widerstandsänderungen an den Kontakten 
100 Ladungstransportmessungen von der atomaren bis zur mesoskopischen Skala auf epitaktischem Graphen

schwankt. Jedoch variiert auch die Thermospannungsdifferenz $\Delta U_{\mathrm{Th}, \mathrm{Diff}}=U_{\mathrm{Th}, \mathrm{ML}}-$ $U_{\mathrm{Th}, \mathrm{BL}}$ zwischen ein- und zweilagigem Graphen. So beträgt in Bereich $\mathrm{A} \Delta U_{\mathrm{Th}, \text { Diff }} \approx 320$ $\mu \mathrm{V}$, in Bereich B $\Delta U_{\mathrm{Th}, \text { Diff }} \approx 0 \mu \mathrm{V}$ und in Bereich C $\Delta U_{\mathrm{Th}, \text { Diff }} \approx-250 \mu \mathrm{V}$.

Aus diesen Beobachtungen kann gefolgert werden, dass die lokale Zustandsdichte der Spitze somit einen erheblichen Einfluss sowohl auf den gemessenen Absolutbeitrag der Thermospannung als auch auf die Differenz des Thermospannungssignals zwischen einund zweilagigem Graphen hat. Die Angabe eines Seebeck-Koeffizienten der Tunnelbarriere sowie dem Übergang zwischen ein- und zweilagigem Graphen, welcher in parallel zu dieser Arbeit entstandenen Veröffentlichung von Park et. al. zu $42 \mu \mathrm{V} / \mathrm{K}$ und 9,6 $\mu \mathrm{V} / \mathrm{K}$ bestimmt worden ist [113], scheint ohne die genaue Charakterisierung der lokalen Zustandsdichte der Spitze daher nicht sinnvoll zu sein. Ohne die genaue Kenntnis der lokalen Zustandsdichte der Spitze muss wiederum zur Bestimmung von durch den Elektronentransport hervorgerufenen Spannungsabfällen an Übergängen zwischen ein- und zweilagigem Graphen immer das elektrochemische Potential für entgegengesetzte Stromrichtungen aufgenommen werden.

Die beobachteten Eigenschaften der Thermospannung haben auch einen direkten Einfluss auf die quantitative Auswertung und Interpretation des durch den Elektronentransport hervorgerufenen Spannungsabfalles an der Grenzfläche zwischen ein- und zweilagigem Graphen. Abbildung 54 (f) zeigt den aus (c) und (e) bestimmten Potentialabfall $U_{\text {Trans }}$ über diesen Bereich der Probe nach Differenzbildung. Die unterschiedlichen Thermospannungsanteile heben sich in erster Ordnung auf. Dies gilt ebenso für die Thermospannungsoszillationen auf zweilagigen Graphen, deren Ursache und Eigenschaften in Kapitel 4.2 beschrieben worden sind. In den folgenden Kapiteln wird zur Analyse der Transportdaten nach der hier vorgestellten Methode der Thermospannungsanteil $U_{\mathrm{Th}}$ entfernt.

Die durch das STM konstruktionsbedingt auftretende Thermospannung in den Transportexperimenten hat auch einen Einfluss auf die räumliche und energetische Auflösung der durch den elektrischen Transport hervorgerufenen Variation im bestimmten lokalen elektrochemischen Potential bzw. $U_{\text {Trans }}$, wie im Folgenden an einer höchstaufgelösten Nullmessung, also $U_{\text {Trans }}=0 \mathrm{~V}$ demonstriert werden soll. Wie in Kapitel 3.5 gezeigt, kann mit 
dem Verfahren der hier verwendeten Rastertunnelpotentiometrie experimentell ein Auflösungsvermögen von 3,7 $\mu \mathrm{V}$ erreicht werden. Prinzipiell unterscheidet sich die apparative Auflösung beim Anlegen einer Querspannung dabei nicht. Durch den hohen Thermospannungsanteil bei den Tieftemperaturmessungen ist man, wie oben gezeigt, zur korrekten Bestimmung von $U_{\text {Trans }}$ auf die Differenzbildung zweier Datensätze mit entgegengesetzter Stromrichtung angewiesen. Jedoch kann bei der hier verwendeten Apparatur ein und derselbe Ort durch Nichtlinearitäten in der Piezo-Ansteuerung sowie thermischer Drift nicht exakt zweimal hintereinander bestimmt werden. Dies hat zur Folge, dass der Thermospannungsanteil bei der Differenzbildung von zwei Potentiometriedaten nicht vollständig kompensiert wird, wie in Abbildung 55 anhand einer Thermospannungsmessung eines $50 \times 34 \mathrm{~nm}^{2}$ großen Bereiches von zweilagigem Graphen gezeigt ist.

Abbildung 55 (a) zeigt die Topographie der Hin- und Rückspur sowie dessen Differenz, Abbildung 55 (b) die für beide Spuren aufgenommene Thermospannung $U_{\mathrm{Th},+}$ und $U_{\mathrm{Th},-}$ und deren gemittelte Differenz $\left(U_{\mathrm{Th},+}-U_{\mathrm{Th},+}\right) / 2$. Laterale Schnitte durch alle Datensätze sind in Abbildung 55 (c) dargestellt. Werden identische Orte innerhalb der beiden Topographie-Spuren gut zur Deckung gebracht, äußert sich dies durch eine minimale Differenz, wie an den lateralen Schnitten aus Abbildung 55 (a) erkennbar ist (durch schwarze Pfeile markiert). In solchen Bereichen ist die Standardabweichung von $U_{\text {Trans }}$ minimal und beträgt $18 \mu \mathrm{V}$ in den Potentiometrie-Daten, wie in Abbildung (b) im Differenzbild der Thermospannungsdaten in Bereich A bestimmt worden ist. Bei einer schlechteren räumlichen Korrelation der Hin- und Rückspur erhält man höhere Werte von hier z.B. $44 \mu \mathrm{V}$ im Bereich B. Dies bedeutet, dass in der vorliegenden verwendeten Apparatur-Konfiguration das Auflösungsvermögen in der Bestimmung der lokalen Transportfelder $U_{\text {Trans }}$ bis um eine Größenordnung schlechter ist als im Vergleich zu thermospannungsfreien Messungen bei Raumtemperatur. 
102 Ladungstransportmessungen von der atomaren bis zur mesoskopischen Skala auf epitaktischem Graphen

a)
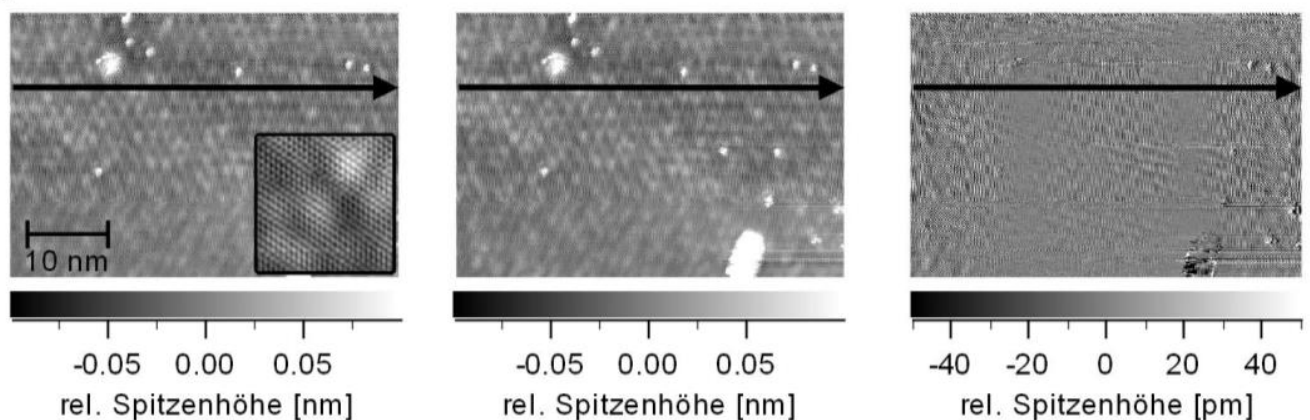

rel. Spitzenhöhe $[\mathrm{nm}]$

rel. Spitzenhöhe $[\mathrm{pm}]$

b)

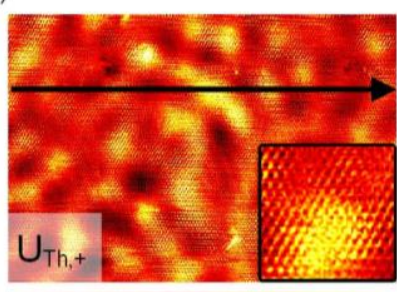

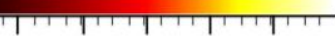

$\begin{array}{lllll}-0.2 & -0.1 & 0.0 & 0.1 & 0.2\end{array}$

Potential [mV]

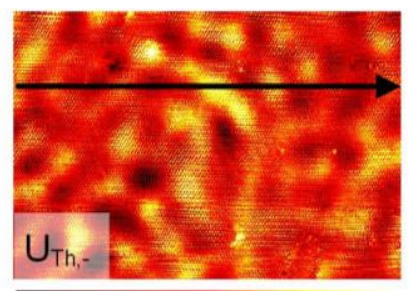

$\begin{array}{lllll}-0.2 & -0.1 & 0.0 & 0.1 & 0.2\end{array}$ Potential [m $\mathrm{m}]$

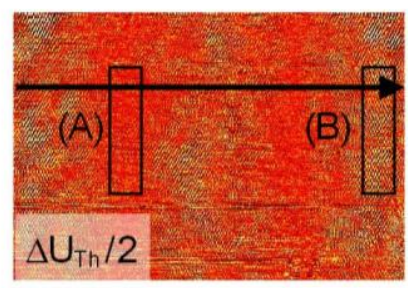

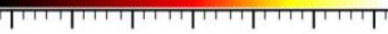

$\begin{array}{lllllll}-75 & -50 & -25 & 0 & 25 & 50 & 75\end{array}$

Potential $[\mu \mathrm{V}]$

c) _ Topographie Hinspur _ _ Topographie Rückspur — Differenz

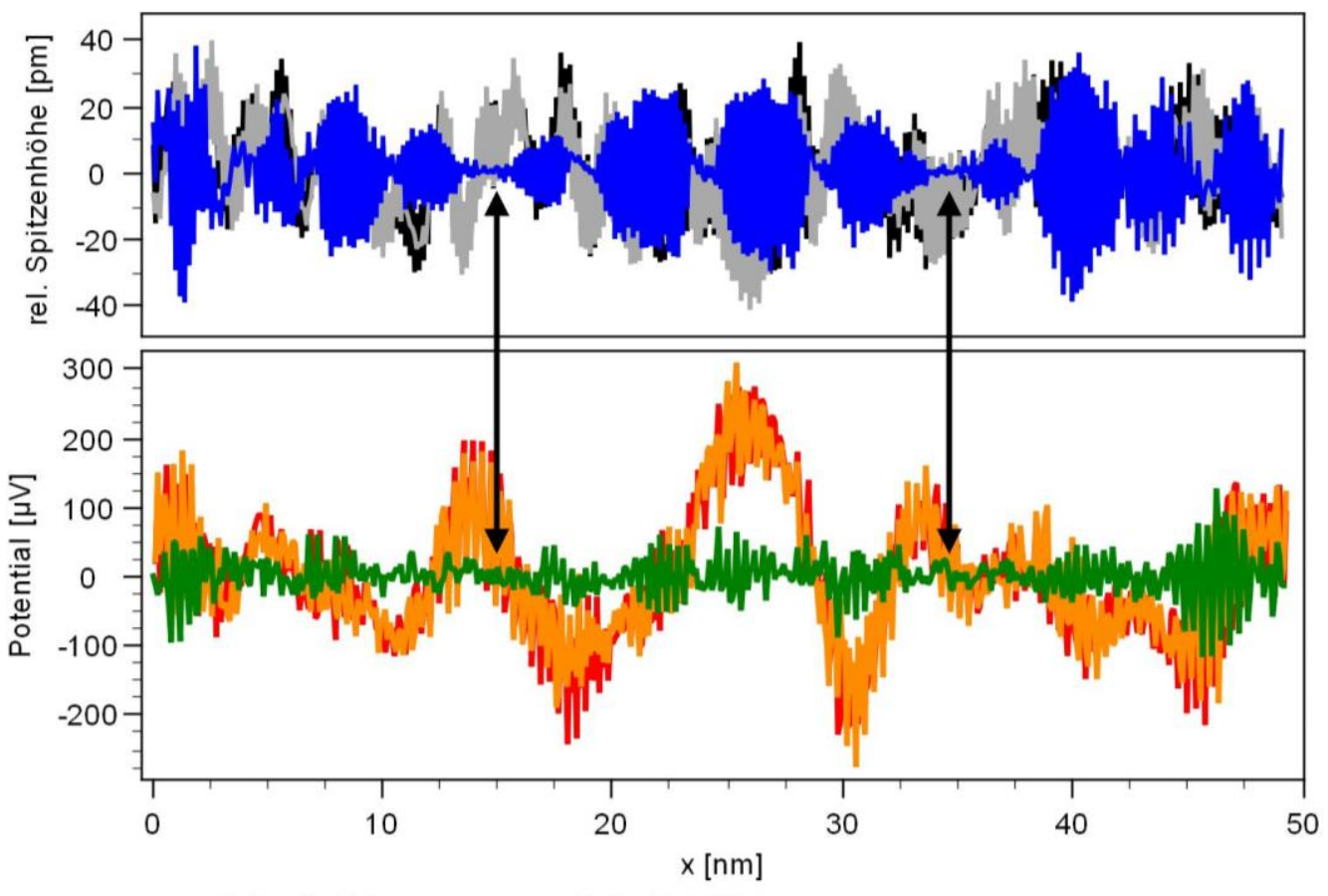

Potential Hinspur _ Potential Rückspur _ Differenz

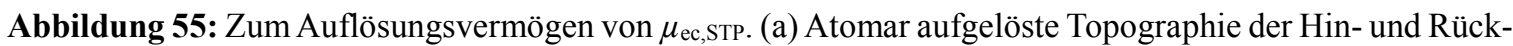
spur sowie deren Differenz (v. 1. n. r), $\left(I_{\mathrm{T}}=0,35 \mathrm{nA}, U_{\mathrm{Bias}}=-50 \mathrm{mV}, 504 \times 346\right.$ Pixel) sowie in (b) für beide Spuren aufgenommen Thermospannung $U_{\mathrm{Th},+,-.}$ (c) Laterale Schnitte entlang der schwarzen Pfeile der in (a) und (b) gezeigten Datensätze. Durch die räumlich nicht exakte Überlagerung der Messungen kommt es zu einem verminderten Auflösungsvermögen bei der Bestimmung von $\mu_{\mathrm{ec}, \mathrm{STP}}$. 


\subsection{Streubeiträge und quantitative Analyse}

Um eine genauere Analyse der Streuprozesse auf kürzeren Längenskalen vornehmen zu können, wurden Transportmessungen auf kleineren Längenskalen mit einer höheren lateralen Auflösung aufgenommen. Abbildung 56 (a) zeigt einen Topographiebereich, in dem sowohl ein- als auch zweilagiges Graphen vorhanden ist. Abbildung 56 (b) zeigt den mittleren Bereich aus (a), dessen Auflösung ungefähr um einen Faktor vier höher ist. In der Topographie sind die verschiedenen Bereiche von ein- und zweilagigem Graphen gekennzeichnet, sowie deren Übergänge des Typs I und II mit und ohne darunterliegender SiCSubstratstufe. Ebenso erkennt man in Abbildung 56 (a) und (b) Höhenvariationen auf den zweilagigen Graphenflächen in der Größenordnung von 10 bis 20 pm, welche durch Wrinkles, wie sie in Kapitel 3.5.3 vorgestellt worden sind, verursacht werden. Die zu beiden Topographien aufgenommenen Potentiometrie-Daten sind in Abbildung 56 (c) und (d) zu sehen. Es treten sowohl auf ein- als auch auf zweilagigem Graphen Spannungsabfälle in der Größenordnung von 50 bis $100 \mu \mathrm{V}$ auf $100 \mathrm{~nm}$ auf und erscheinen räumlich homogen. Stark lokalisierte Spannungsabfälle sind an den Übergängen zwischen ein- und zweilagigem Graphen auszumachen, welche in der Größenordnung von $100 \mu \mathrm{V}$ liegen. Bei genauerer Betrachtung sind ebenso an einigen Wrinkels, wie sie in Abbildung 53 durch schwarze Pfeile markiert sind, Spannungsabfälle von 10 bis $20 \mu \mathrm{V}$ auszumachen. Die stellenweise leichten periodischen Modulationen in Abbildung 56 (d) kommen durch die in Kapitel 5.2.1 beschriebene unvollständige Eliminierung des Thermospannungsanteils zustande. Im Folgenden soll die quantitative Bestimmung der spezifischen Widerstände lokalisierter Defekte sowie der auftretenden freien Flächen von ein- und zweilagigem Graphen vorgestellt werden. Als Grundlage wird angenommen, dass sich die mit der Rastertunnelpotentiometrie untersuchten Bereiche nach dem ohmschen Gesetz verhalten. Dies beinhaltet für die im Folgenden vorgestellten Methoden, dass sowohl ein- und zweilagiges Graphen eine über die Probe isotrope spezifische Leitfähigkeit $\rho_{M L}$ und $\rho_{B L}$ besitzt und sich verschiedene Stufen zwischen ein- und zweilagigem Graphen jeweils durch einen spezifischen Linienwiderstand beschreiben lassen. Zusätzlich wird die geometrische Struktur der gemessenen Bildbereiche und die damit einhergehende inhomogene Stromdichte berücksichtigt. 
104 Ladungstransportmessungen von der atomaren bis zur mesoskopischen Skala auf epitaktischem Graphen

a)

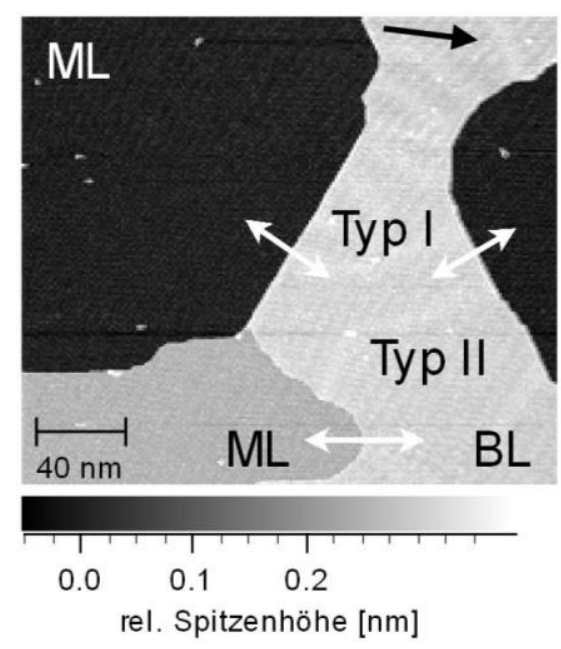

c)

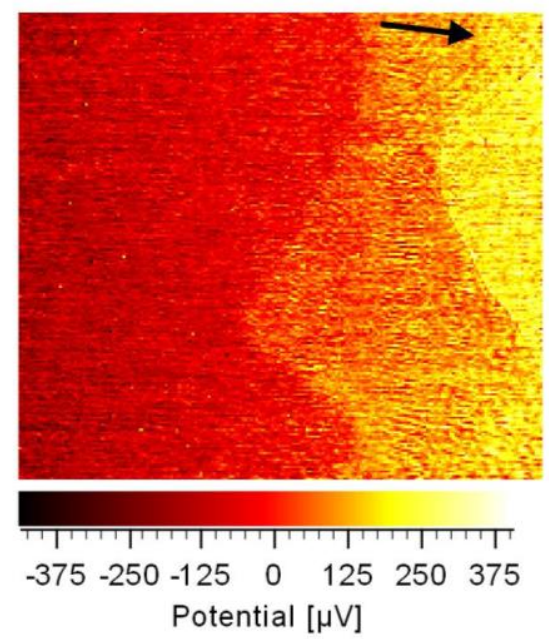

b)

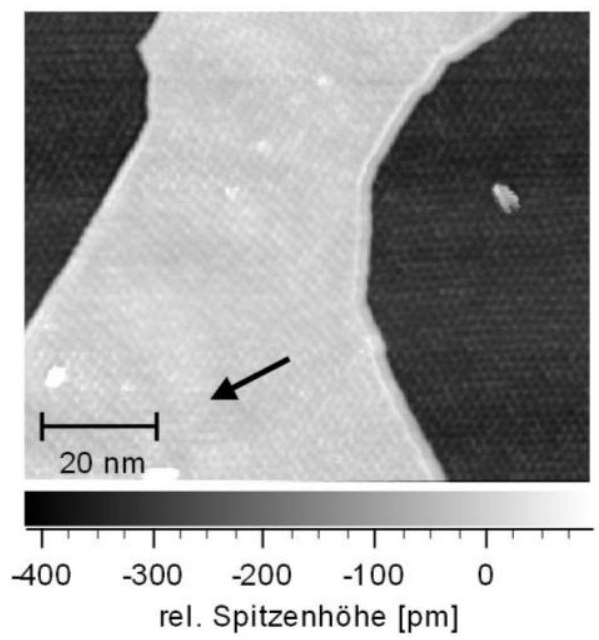

d)

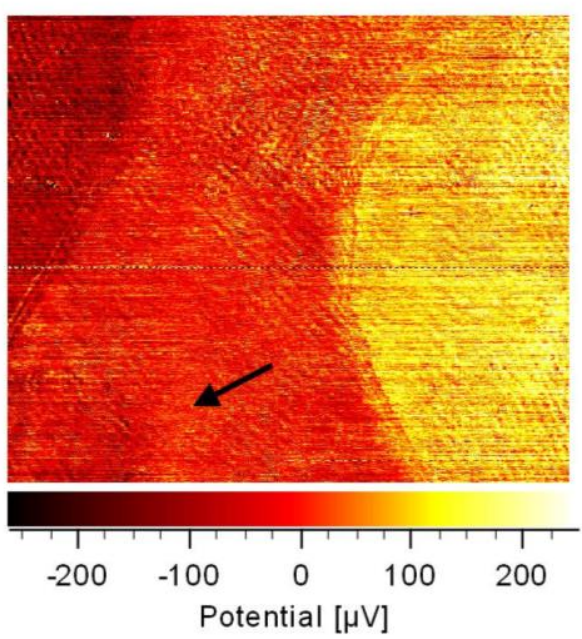

Abbildung 56: (a) Topographiebereich mit ein- und zweilagigen Graphenbedeckung zur Analyse der verschiedenen Streubeiträge $\left(236 \times 252 \mathrm{~nm}^{2}, 200 \times 200\right.$ Pixel, $\left.U_{\text {Bias }}=30 \mathrm{mV}, I_{\mathrm{T}}=175 \mathrm{pA}\right)$. (b) Bildbereich aus (a) aufgenommen mit einer höheren Auflösung $\left(98 \times 81 \mathrm{~nm}^{2}, 512 \times 512\right.$ Pixel. (c, d) Potentiometrie-Daten zu (a) bzw. (b) bei einer makroskopischen Stromdichte von $j_{\text {makro }}=|2,9 \mathrm{~A} / \mathrm{m}|$. Sowohl ein- als auch zweilagiges Graphen zeigen einen Spannungsabfall auf den freien Flächen. Ebenso sind lokalisierte Spannungsabfälle an den beiden verschiedenen Grenzflächen des Typs I und II zwischen ein- und zweilagigem Graphen erkennbar. Schwarze Pfeile markieren Wrinkles, welche ebenso zu Spannungsabfällen auf zweilagigem Graphen führen. 


\subsubsection{Selbstkonsistente Modellierung im Rahmen des ohmschen Gesetzes}

In diesem Abschnitt soll überprüft werden, in wie weit sich die experimentell erhaltenen Potentialdaten durch das mikroskopische, lokale ohmsche Gesetz konsistent beschreiben lassen oder aber Abweichungen zu diesem Modell bestehen. Dazu wird die geometrische Struktur der untersuchten Bereiche mit berücksichtigt. Es wird angenommen, dass der zu berechnende Probenbereich bis auf die Ränder stromquellenfrei ist und somit $\nabla \cdot \boldsymbol{j}=0$ gilt und die lokale Stromdichte $\boldsymbol{j}(x, y)$ durch das ohmsche Gesetzt $\boldsymbol{j}(x, y)=$ $-\sigma(x, y) \nabla \mu_{e c}(x, y)$ als Antwort auf einen Gradienten im lokalen elektrochemischen Potential und der lokalen Leitfähigkeit $\sigma(x, y)$ der verschiedenen Flächen und Defekte gegeben ist. Dieses Problem kann auf ein diskretes zweidimensionales Gitter von klassischen ohmschen Widerständen übertragen und mit dem Knotenpotentialverfahren gelöst werden. Dabei wird jedem Knotenpunkt des kubischen Gitters ein Potentialwert und den Verbindungslinien zu den benachbarten Knotenpunkten ein Widerstand bzw. Leitwert zugeordnet. Die Anzahl der diskreten Punkte auf dem Gitter ist so gewählt, dass sie der experimentellen Auflösung der gemessenen Potentialwerte entspricht. In dem Modell wird dabei ein- und zweilagigem Graphen jeweils ein isotroper Flächenwiderstand $\rho_{\mathrm{ML} / \mathrm{BL}}$ sowie den verschiedenen Übergängen ein eindimensionaler spezifischer Linienwiderstand $\rho_{\text {Defekt }}$ zugeordnet. Der Widerstand eines Bildbereiches der Rasterweite $l_{\text {Pixel }} \cdot l_{\text {Pixel }}$ ist dann für die freien Flächen gegeben über $R_{\mathrm{ML} / \mathrm{BL}}=\rho_{\mathrm{ML} / \mathrm{BL}} \cdot l_{\text {Pixel }} / l_{\text {Pixel }}=\rho_{\mathrm{ML}, \mathrm{BL}}$ und für die Übergänge definiert als $R_{\text {Defekt }}=\rho_{\text {Defekt }} \cdot l_{\text {Pixel }}^{-1}+\rho_{\mathrm{ML} / \mathrm{BL}}$. Um für ein gegebenes Widerstandsnetzwerk die Potentialverteilung bei gegebenen Randbedingungen zu erhalten, wird die Lösung des linearen Widerstandsnetzwerkes nach einer Implementierung von J. Homoth in die MatlabProgrambibliothek ermittelt [132]. Die Identifikation der verschiedenen freien Flächen sowie Stufenkanten werden anhand der Topographie ermittelt. Die laterale Position eines Überganges ist durch die halbe Höhe eines Übergangs definiert. Für die exakte Position der Kanten bzw. Übergänge wird eine Kantensuchroutine aus Matlab verwendet. Die Genauigkeit der Position der ML-BL-Übergänge bzw. ML-ML-Stufen kann dabei mit \pm einem Pixel angegeben werden. Die horizontalen Ränder der Bildbereiche werden gemäß Dirichletscher Randbedingungen entsprechend der experimentell mit Hilfe der Rastertunnel-potentiometrie erhaltenen Potentialdaten auf den Mittelwert der ersten bzw. letzten Spalte gesetzt. Die vertikalen Ränder erhalten anhand der experimentellen Daten linearisierte 
106 Ladungstransportmessungen von der atomaren bis zur mesoskopischen Skala auf epitaktischem Graphen

Potentialwerte oder aber von-Neumann-Randbedingungen ${ }^{4}$. Anschließend werden die Widerstände bestimmt, bei denen die Summe der Abweichungsquadrate zwischen der Potentialverteilung der Simulation und dem Messergebnis minimal wird. Dazu wird ein restringierter direkter Suchalgorithmus nach der Simplexmethode von Nelder und Mead verwendet, welcher in der Programmbibliothek von Matlab integriert ist [133]. Für die Stufenwiderstände wird jeweils ein Suchintervall zwischen $1 \Omega \mu \mathrm{m}$ und $400 \Omega \mu \mathrm{m}$ und für die Flächenwiderstände ein Suchintervall zwischen $30 \Omega / \square$ und $8000 \Omega / \square$ zugelassen.

Dieses Verfahren liefert in den angegeben Grenzen das optimale Verhältnis der gesuchten Widerstände zu der experimentell gegebenen Potentiallandschaft. Um quantitative Werte zu erhalten, werden im Anschluss die Widerstandswerte so skaliert, dass die mittlere Stromdichte des Bildes der experimentell ermittelten makroskopischen Stromdichte entspricht.

Abbildung $57(\mathrm{a}, \mathrm{b})$ zeigt eine $288 \times 186 \mathrm{~nm}^{2}$ große Topographie und das dazu aufgenommene, thermospannungsbereinigte Potentiometriesignal. Der Bildbereich enthält eine zweilagige Graphen-Terrasse, die durch ML-BL-Übergänge des Typs I und II in umliegendes einlagiges Graphen eingebettet ist. Eine ML-ML-Stufe verläuft von links nach rechts nahezu horizontal bis zur Mitte des Bildes. Abbildung 57 (c) zeigt das Simulationsergebnis mit den spezifischen Flächenwiderständen $\rho_{\mathrm{ML}}=527 \Omega / \square, \rho_{\mathrm{BL}}=411 \Omega / \square$ und Stufen bzw. Übergangswiderständen von $\rho_{\mathrm{ML}-\text { Typ I }}=9 \Omega \mu \mathrm{m}, \rho_{\mathrm{ML} / \mathrm{BL}-\text { Typ I }}=42 \Omega \mu \mathrm{m}$ und $\rho_{\mathrm{ML} / \mathrm{BL}-\mathrm{Typ} \text { II }}=50 \Omega \mu \mathrm{m}$. Die berechnete Potential-verteilung gibt das experimentell bestimmte Potentiometrie-Signal in allen Bildbereichen gut wieder. Abbildung 57 (d) zeigt die Differenz zwischen $\mu_{\mathrm{ec}, \mathrm{STP}}(x, y)$ und den simulierten Daten. Der Mittelwert der Absolutdifferenzen beträgt $72 \mu \mathrm{V}$.

\footnotetext{
${ }^{4}$ Für die einzelnen simulierten Bilder sind verschiedene Randbedingungen verwendet worden, abhängig von der geometrischen Struktur des Bildbereiches und der bestmöglichen Anpassung.
} 
a)

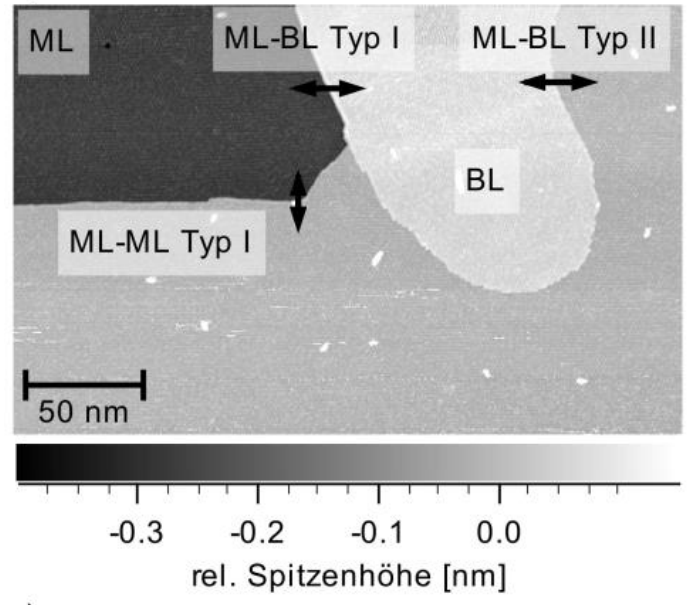

c)

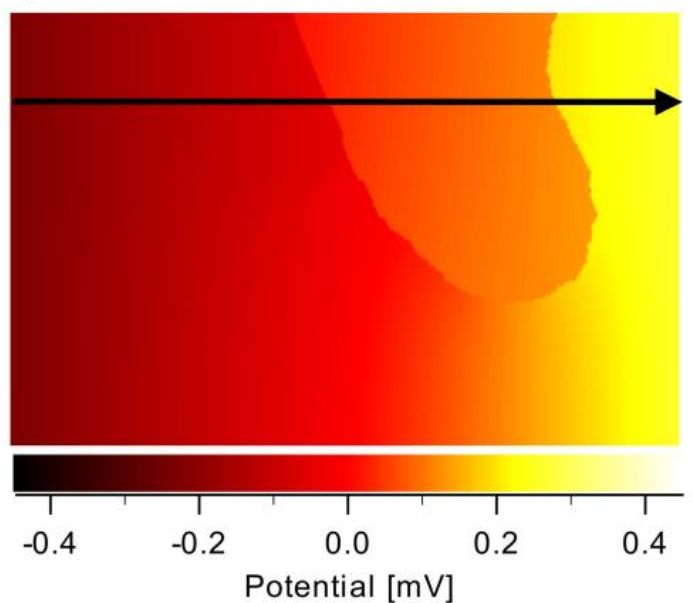

e)

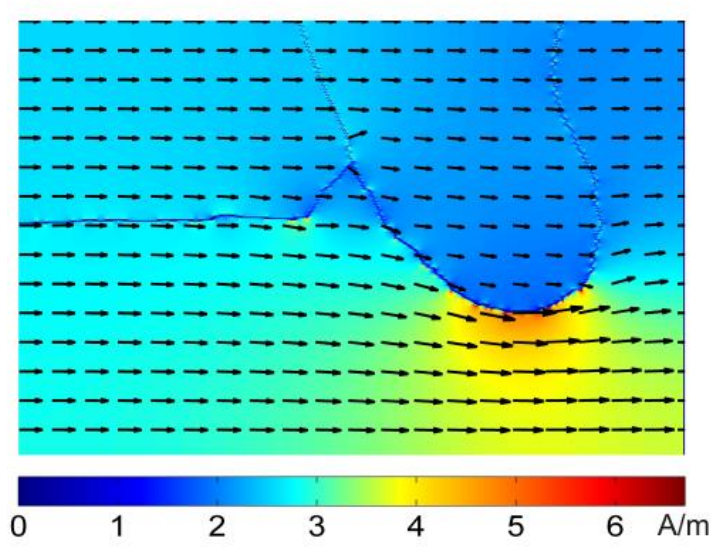

b)

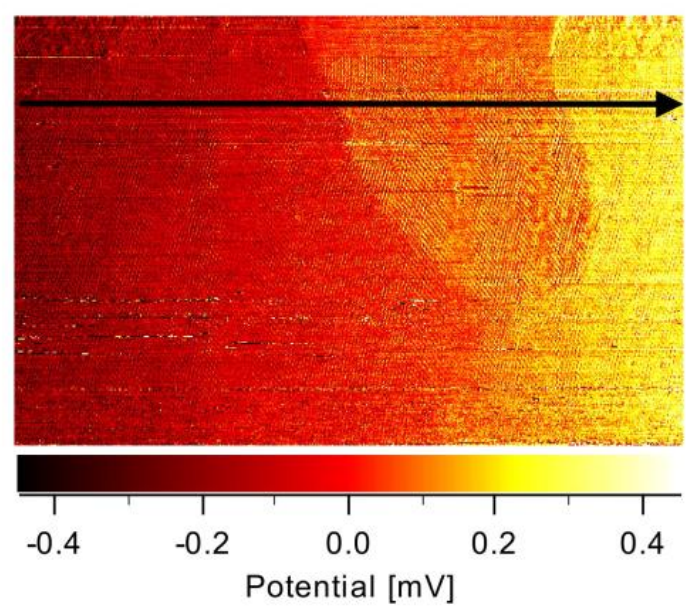

d)

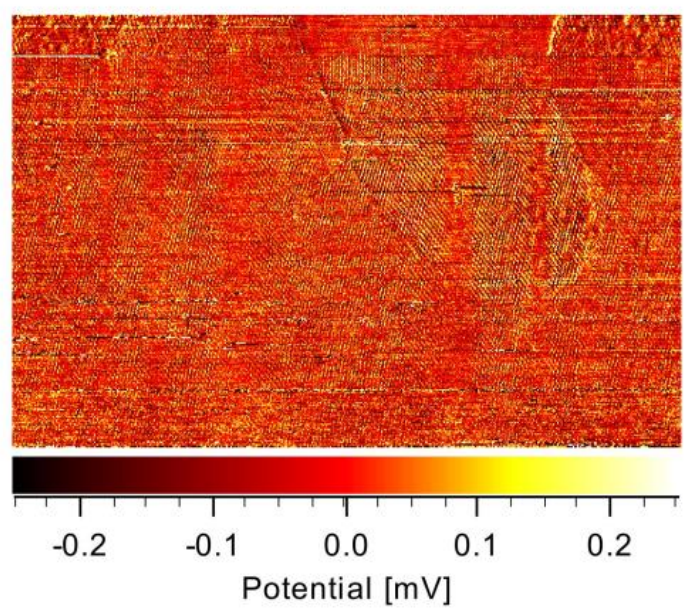

f)

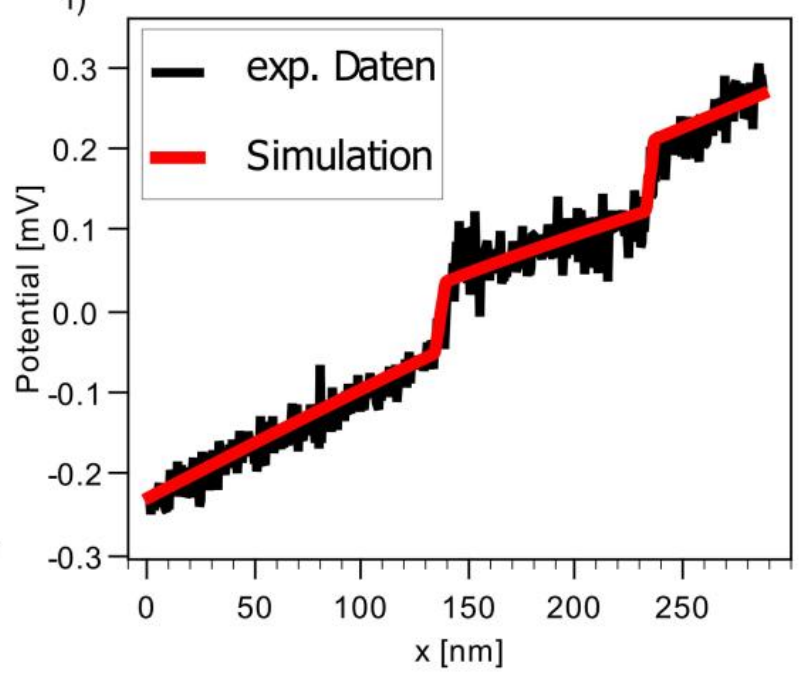

Abbildung 57: (a, b) Topographie und thermospannungsbereinigtes Potentiometriesignal $\left(288 \times 186 \mathrm{~nm}^{2}\right.$, $506 \times 372$ Pixel, $I_{\mathrm{T}}=0,3 \mathrm{nA}, U_{\text {Bias }}=40 \mathrm{mV}, j_{\text {makro }}=2,8(2) \mathrm{A} / \mathrm{m}$ ). (c) Berechnete Potentialverteilung. (d) Differenz der berechneten und experimentell bestimmten Potentialverteilung. (e) Betragsquadrat der Stromdichte und Vektorplot. Die Stromdichte variiert zwischen 0 und 6,7 A/m (f) Lateraler gemittelte Schnitte entlang der in (b) und (c) durch Pfeile markierten Bereiche. 
108 Ladungstransportmessungen von der atomaren bis zur mesoskopischen Skala auf epitaktischem Graphen

Ein Vergleich der lateralen Schnitte über den in Abbildung 57 (b) und (c) markierten Bereich, welche horizontal über die Fermiwellenlänge gemittelt worden sind, zeigt, dass der Gradient auf den freien Flächen als auch die Höhe der Potentialsprünge an den Übergängen zwischen ein- und zweilagigem Graphen gut getroffen werden.

Auf Grundlage dieser Modellierung lässt sich ebenso die lokale Stromdichte an der Oberfläche der Struktur berechnen und wie in Abbildung 57 (e) darstellen. Das Betragsquadrat der Stromdichte ist farbkodiert abgebildet, der überlagerte Vektorplot gibt die Richtung der Stromdichte an. Es wird sichtbar, dass die Stromdichte aufgrund der gegebenen Topologie und Defekte innerhalb des Bildes im Vergleich zur makroskopischen Stromdichte in diesem Fall bis zu einem Faktor zwei variiert. Dies zeigt, dass für eine quantitative Analyse im Rahmen eines mikroskopischen ohmschen Gesetzes für die gegebenen Topologien die selbstkonsistente Berechnung nötig ist.

Zur quantitativen Bestimmung der einzelnen spezifischen Leitfähigkeiten wurden für verschiedene Regionen die Potentialverteilungen berechnet. Eine detaillierte Darstellung ist im Anhang dieser Arbeit zu finden. Abbildung 58 (a) und (b) fasst die Ergebnisse für die spezifischen Widerstände der einzelnen Übergänge sowie der freien Flächen zusammen. Die Mittelwerte der einzelnen Übergänge betragen $\rho_{\mathrm{ML}-\mathrm{BL}-\mathrm{I}}=(43 \pm 18) \Omega \mu \mathrm{m}$, $\rho_{\mathrm{ML}-\mathrm{BL}-\mathrm{II}}=(80 \pm 70) \Omega \mu \mathrm{m}, \rho_{\mathrm{ML}-\mathrm{ML}-\mathrm{I}}=(5 \pm 7) \Omega \mu \mathrm{m}, \rho_{\mathrm{ML}-\mathrm{ML}-\mathrm{II}}=(34 \pm 0,2) \Omega \mu \mathrm{m}$. Der Flächenwiderstand von ein- bzw. zweilagigem Graphen beträgt $\rho_{\mathrm{ML}}=(506 \pm 107) \Omega / \square$ und $\rho_{\mathrm{BL}}=(517 \pm 199) \Omega / \square$. Das $95 \%$-Konfidenzintervall der jeweiligen Werte ist in Abbildung 58 dunkelgrau, die jeweilige Standardabweichung hellgrau unterlegt. 

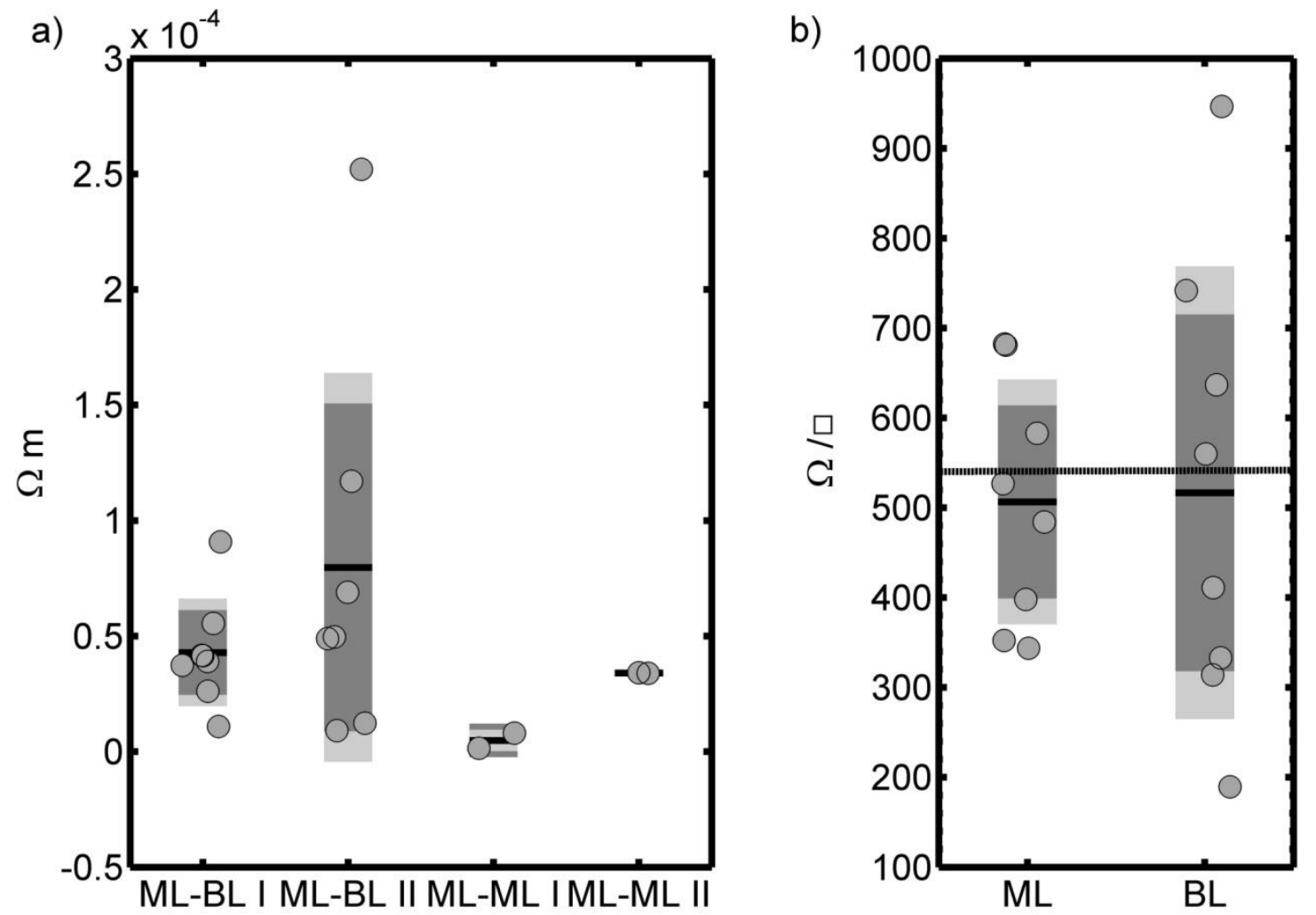

Abbildung 58: (a) Zusammenfassung der anhand der diffusiven Modellierung berechneten spezifischen Widerstände der einzelnen Stufen und (b) freien Flächen von ein- und zweilagigem Graphen. Der Mittelwert der Widerstandswerte der einzelnen Defekte ist jeweils durch schwarze Linien gekennzeichnet, Symbole markieren das Ergebnis aus jeweils einer Abbildung. Die ermittelten Werte weisen sowohl für die Stufenkantenwiderstände wie auch für die freie Fläche eine hohe Streuung auf. Das 95\%- Konfidenzintervall der ermittelten Widerstandswerte ist durch graue Balken dargestellt. Die spezifischen Stufenwiderstände betragen $\rho_{\text {ML-BL I }}$ $=(43 \pm 18) \Omega \mu \mathrm{m}, \rho_{\mathrm{ML}-\mathrm{BL} I \mathrm{II}}=(80 \pm 70) \Omega \mu \mathrm{m}, \rho_{\mathrm{ML}-\mathrm{ML} \mathrm{I}}=(5 \pm 7) \Omega \mu \mathrm{m}, \rho_{\mathrm{ML}-\mathrm{ML} \text { II }}=(34 \pm 0,2) \Omega \mu \mathrm{m}$ und die der freien Flächen $\rho_{\mathrm{ML}}=(506 \pm 107) \Omega / \square$ und $\rho_{\mathrm{BL}}(517 \pm 199) \Omega / \square$. Die Streuung der Daten ist jeweils über die Standardabweichung charakterisiert und beträgt $\sigma_{\mathrm{ML}-\mathrm{BL} \text { I }}=23 \Omega \mu \mathrm{m}, \sigma_{\mathrm{ML}-\mathrm{BL} \text { II }}=84 \Omega \mu \mathrm{m}, \sigma_{\mathrm{ML}-\mathrm{ML} \text { I }}=0,2 \Omega \mu \mathrm{m}$, $\sigma_{\mathrm{ML}}=136 \Omega / \square$ und $\sigma_{\mathrm{BL}}=252 \Omega / \square$.

Ebenso ersichtlich ist, dass auf Längenskalen von einigen $10 \mathrm{~nm}$ bis $100 \mathrm{~nm}$ räumliche Bereiche existieren, die unabhängig von der Wahl der Randbedingungen Abweichungen des Potentialverlaufes im Vergleich zu der ohmschen Modellierung aufweisen. Dies ist am folgenden Datensatz in Abbildung 59 gezeigt. Die Topographie zeigt eine sechseckige Insel zweilagigen Graphens, welche von einlagigem Graphen umschlossen ist. Im oberen rechten Bildrand ist eine doppelte Substratstufe zu sehen (ML-ML-Typ II). Der experimentelle Spannungsverlauf ist in Abbildung 59 (b), das simulierte Potential in Abbildung 59 (c) dargestellt. Die Differenz zwischen experimentellen und simulierten Daten ist in Abbildung 
110 Ladungstransportmessungen von der atomaren bis zur mesoskopischen Skala auf epitaktischem Graphen

59 (d) zu sehen. Um kurzreichweitige Variationen in der Potentialdifferenz zwischen experimentellen und simulierten Daten zu unterdrücken, wurde das Differenzbild mit einem Boxfilter von $5 \times 5$ Pixeln entsprechend einer Fläche von $5 \mathrm{~nm}^{2}$ gemittelt. So erstreckt sich im Differenzbild z.B. im unteren Bildbereich ein Probenbereich, welcher einige $10 \mu \mathrm{V}$ unter dem durch die Modellierung erwartetem Wert liegt.

a)

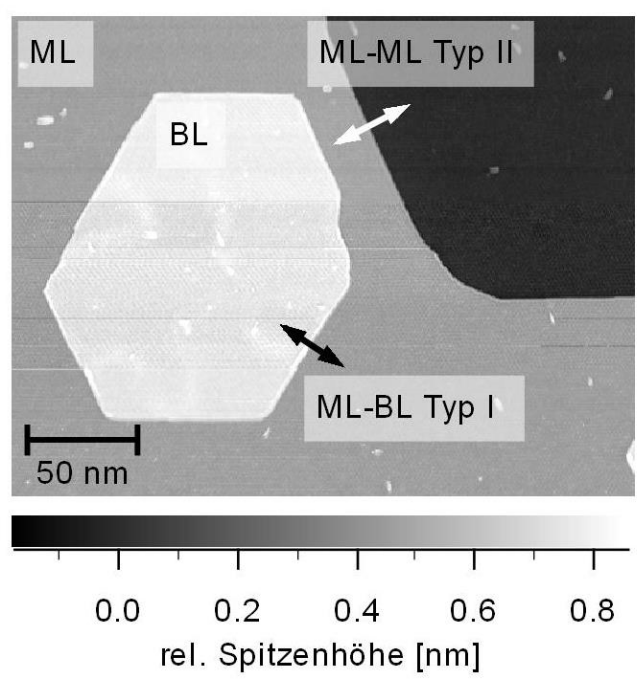

c)

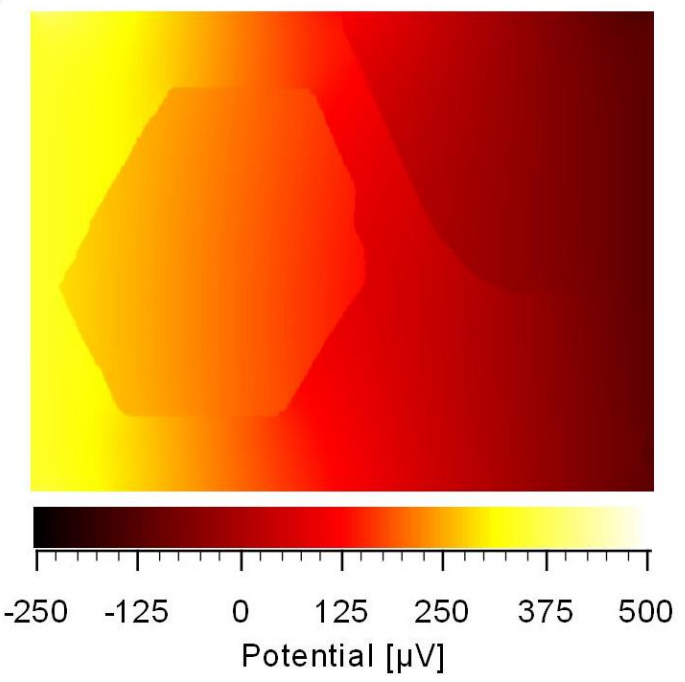

b)

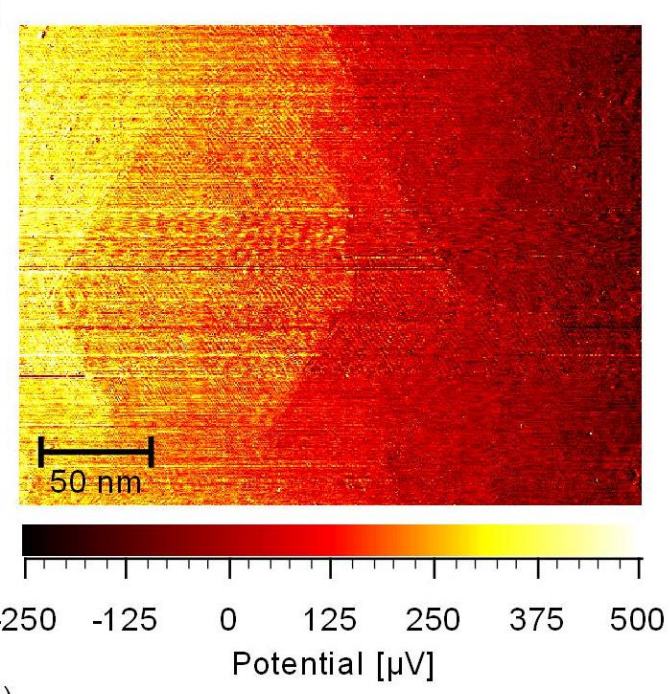

d)

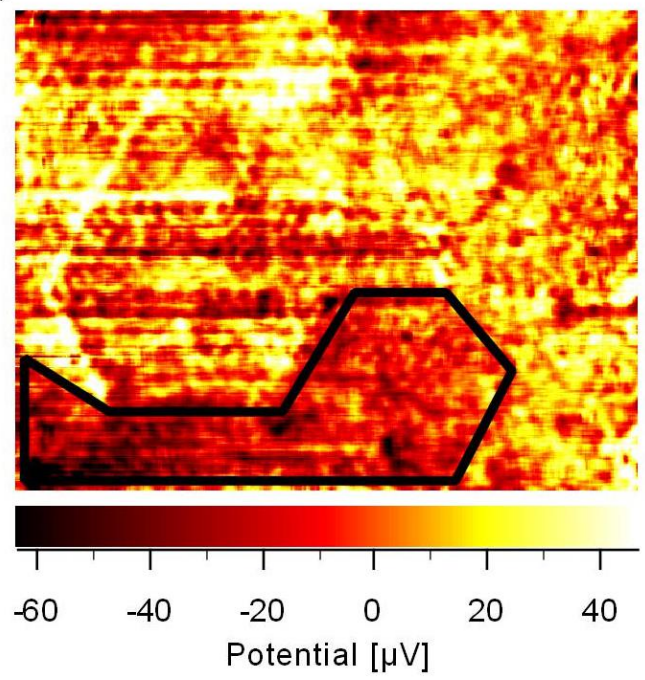

Abbildung 59: (a) Topographie und (b) thermospannungsbereinigtes Potentiometriesignal $\left(287 \times 216 \mathrm{~nm}^{2}\right.$, $504 \times 433$ Pixel, $I_{T}=0,3 \mathrm{nA}, U_{\text {Bias }}=40 \mathrm{mV}, j_{\text {makro }}=3,2(2) \mathrm{A} / \mathrm{m}$ ). (c) Berechnete Potentialverteilung. (d) Räumlich über $5 \mathrm{~nm}^{2}$ gemittelte Differenz der berechneten und experimentell bestimmten Potentialverteilung $(5 \times 5$ Pixel Box-Filter). Es sind langreichweitige Abweichungen zwischen simulierten und gemessenen Potentialwerten zu erkennen. 
Auffällig bei der hier vorgestellten Analyse ist die breite Verteilung der gefundenen Widerstandswerte. So liegen abhängig vom simulierten Bildausschnitt z.B. für ein- und zweilagiges Graphen die Widerstände für die Flächen im Intervall von $340 \Omega / \square$ bis 680 bzw. 190 $\Omega / \square$ bis sogar $950 \Omega / \square$. Stufenwiderstände der ML-BL-Übergänge sind ebenso in einem großen Intervall zwischen $10 \Omega \mu \mathrm{m}$ und $90 \Omega \mu \mathrm{m}$ für den Typ I Übergang und zwischen $9 \Omega \mu \mathrm{m}$ und $250 \Omega \mu \mathrm{m}$ für den Typ II zu finden. Es kann zwar ein Mittelwert angegeben werden, dieser charakterisiert offensichtlich aber nicht die mikroskopischen Transporteigenschaften der einzelnen Stufen.

Für die breite Verteilung der ermittelten Transportgrößen der ML-BL-Übergänge des Typs I und II sowie der freien Flächen (vgl. Abbildung 58) wie auch lokalen Abweichungen kommen verschiedene Ursachen in Frage. Zum einen besteht die Möglichkeit, dass die ohmsche Modellierung bei diesem Probensystem gerechtfertigt, aber die getroffenen Annahmen nicht ausreichend sind. So werden Defekte wie die in Kapitel 3.5.3 identifizierte Versetzung in der Zwischenschicht sowie Wrinkels und damit einhergehende Verspannungen der umliegenden Graphenbereiche in der Simulation nicht berücksichtigt, da diese aufgrund ihrer topographischen Eigenschaften nur schwer identifizierbar sind und sich bisher nicht eindeutig klassifizieren ließen. Zum anderen stellt sich die grundsätzliche Frage, ob auf diesen Längenskalen das hier vorgestellte Modell des ohmschen Ladungstransportes richtig ist, oder aber ein anderes Transportmodell die bessere Wahl zur Beschreibung des Verlaufes des elektrochemischen Potentials ist, welches z.B. Quanteninterferenzeffekte mit berücksichtigt. Aus quantenmechanischer Sicht wird eine von der Geometrie der Stufe als auch vom Winkel der auftreffenden Elektronen an einen ML-BL-Übergang abhängige Transmission erwartet $[122,134,135]$. Eine experimentelle Bestätigung konnte hierfür bisher jedoch nicht gegeben werden. Außerdem werden bei den theoretischen Berechnungen spezifische Eigenschaften des darunterliegenden Substrates nicht berücksichtigt. Dabei werden in der Regel zwei Stufentypen diskutiert, die gestaffelten (engl. armchair) und die gezackten (engl. zickzag). In epitaktischem Graphen auf $\mathrm{SiC}(0001)$ treten überwiegend gestaffelte Graphenkanten auf $[136,137]$ wie in Abbildung 59 an der hexagonalen zweilagigen Grapheninsel zu erkennen ist. Es kommen aber auch, wie in Abbildung 58 (a) zu sehen, beliebige andere Orientierungen vor. Innerhalb der eigenen Daten findet sich keine Korrelation zwischen der Stufenkantenorientierung und dem Widerstand einzelner Stufen. Ebenso ist vorstellbar, dass die idealisierte Vorstellung von selbst zwei zu bestimmenden von der Orientierung abhängigen Stufenwiderständen an z.B. ML-BL-Übergängen des 
112 Ladungstransportmessungen von der atomaren bis zur mesoskopischen Skala auf epitaktischem Graphen

Typs I nicht weitreichend genug ist. Aufgrund der starken Unordnung und Einbettung der Stufen in die Umgebung, ist es ebenso denkbar, dass in epitaktischem Graphen die Transmission jeder einzelnen Stufe von ihrer individuellen atomaren Struktur abhängt und dies die breite Verteilung der erhaltenen Widerstandswerte erklärt.

\subsubsection{Diskussion und Einordnung in bisherige Ladungstransportuntersuchungen}

Neben den hier vorgestellten Ergebnissen wurden auf epitaktischem Graphen verschiedene Transportstudien auf mesoskopischen Längenskalen zur Bestimmung von Transportgrößen wie spezifischen Stufenwiderständen und Leitfähigkeiten der freien Flächen geführt. Im Folgenden sollen parallel zu dieser Arbeit veröffentlichte Ergebnisse für die freien Flächen von ein- und zweilagigem Graphen und im Anschluss die der Stufenübergänge im Kontext der eigenen Ergebnisse diskutiert werden.

Tabelle 1 fasst die quantitativen Ergebnisse der einzelnen Studien im Kontext der eigenen Ergebnisse zusammen. Allgemein ist ein quantitativer Vergleich der einzelnen Arbeiten schwierig, da es sich zum einen um verschiedene Untersuchungsmethoden und Umgebungen (RT bis $6 \mathrm{~K}$, UHV und Atmosphäre) als auch um verschiedene Proben (4H-SiC, $6 \mathrm{H}-$ $\mathrm{SiC}$ ) und Präparationsmethoden handelt. Im Vergleich zu den bisherigen Veröffentlichungen wird bei der Darstellung der eigenen Widerstandswerte zusätzlich das Intervall, indem sich die Widerstandswerte der eingeteilten Defekte befinden, angegeben. Dadurch soll hier betont werden, dass es sich bei der gefundenen Streuung der Messdaten vermutlich nicht um eine klassische, apparative Messunsicherheit handelt. Es ist eher davon auszugehen, dass eine strikte Einteilung bzw. Katalogisierung in z.B. einen ML-BLStufenkantenwiderstand des Typs I aufgrund der mikroskopisch variierenden Defekt-struktur nicht ausreichend ist.

Ji et al. haben mit Hilfe der Rastertunnelpotentiometrie auf epitaktischem Graphen auf semi-isolierendem $\mathrm{SiC}(0001)$ den Spannungsabfall auf den freien Flächen als auch auf verschiedenen Stufen und Übergängen zwischen ein- und zweilagigem Graphen bei Raumtemperatur und $72 \mathrm{~K}$ untersucht [122]. Sie beobachten ebenso ein lineares Verhalten zwischen Gradienten im elektrochemischen Potential auf den freien Flächen und den 
Spannungsabfällen an lokalisierten Defekten wie Übergängen zwischen ein- und zweilagigem Graphen.

\begin{tabular}{|c|c|c|c|c|c|c|c|c|c|c|}
\hline Verfahren & $\begin{array}{l}\text { Substrat } \\
\text { Polytyp }\end{array}$ & $\begin{array}{c}\mathrm{T} \\
{[\mathrm{K}]}\end{array}$ & $\begin{array}{c}\mathrm{p} \\
{[\mathrm{mbar}]}\end{array}$ & $\begin{array}{c}\mathrm{n} \\
{\left[\mathrm{cm}^{-2}\right]}\end{array}$ & $\begin{array}{c}\rho_{\mathrm{ML}} \\
{[\Omega / \square]}\end{array}$ & $\begin{array}{c}\rho_{\mathrm{BL}} \\
{[\Omega / \square]}\end{array}$ & $\begin{array}{l}\rho_{\mathrm{ML}-\mathrm{BL} I} \\
{[\Omega \mu \mathrm{m}]}\end{array}$ & $\begin{array}{c}\rho_{\mathrm{ML}-\mathrm{BL} \text { II }} \\
{[\Omega \mu \mathrm{m}]}\end{array}$ & $\begin{array}{l}\rho_{\mathrm{ML}-\mathrm{ML}} \\
{[\Omega \mu \mathrm{m}]}\end{array}$ & $\begin{array}{c}\rho_{\text {ML-ML II }} \\
{[\Omega \mu \mathrm{m}]}\end{array}$ \\
\hline STM/STP & $\begin{array}{c}6 \mathrm{H} \\
\text { n-dotiert }\end{array}$ & 6 & $<2 \cdot 10^{-11}$ & $1,3(2) \cdot 10^{13}$ & $\begin{array}{c}340-680 \\
(506 \pm 107)\end{array}$ & $\begin{array}{c}190-950 \\
(517 \pm 199)\end{array}$ & $\begin{array}{c}10-90 \\
(43 \pm 18)\end{array}$ & $\begin{array}{c}9-250 \\
(80 \pm 70)\end{array}$ & $\begin{array}{l}1,5-8 \\
(5 \pm 7)\end{array}$ & $\begin{array}{c}33,9- \\
34,1 \\
(34 \pm 0,2)\end{array}$ \\
\hline $\begin{array}{c}\text { STM/STP } \\
{[122]}\end{array}$ & $\begin{array}{c}6 \mathrm{H} / 4 \mathrm{H} \\
\text { semi- } \\
\text { isol. }\end{array}$ & 72 & k. A. & $\sim 1 \cdot 10^{13}$ & $\sim 200 \pm 25$ & $\sim 200$ & k. A. & $20,9 \pm 5,7$ & k.A. & $6,9 \pm 2,9$ \\
\hline $\begin{array}{c}\text { STM/STP } \\
{[130]}\end{array}$ & $\begin{array}{l}4 \mathrm{H} \\
\text { k.A. }\end{array}$ & 81 & $4 \cdot 10^{-10}$ & $\sim 1 \cdot 10^{13}$ & 833 & 769 & k. A. & $1,65^{5}$ & $1,3^{4}$ & k. A \\
\hline $\begin{array}{c}\text { Hall-Bar } \\
\text { [138] }\end{array}$ & $\begin{array}{c}4 \mathrm{H} \\
\text { semi- } \\
\text { isol. }\end{array}$ & $\begin{array}{c}293- \\
25\end{array}$ & k. A. & k. A. & $\approx 400$ & k. A. & k. A. & k. A. & k. A. & k. A. \\
\hline AFM [134] & $\begin{array}{c}4 \mathrm{H} \\
\text { semi- } \\
\text { isol. }\end{array}$ & 293 & k. A. & k. A. & $640 \pm 136$ & $313 \pm 30$ & k. A. ${ }^{6}$ & k. A. ${ }^{5}$ & k. A. & k. A. \\
\hline
\end{tabular}

Tabelle 1: Vergleich eigener Ergebnisse (grau schraffiert) mit bisherigen quantitativen Ergebnissen von Transportuntersuchungen auf mesoskopischer Längenskale mit Hilfe von Rastersondentechniken und HallBar-Strukturen auf epitaktischem Graphen. Bei den eigenen Daten ist sowohl das Intervall innerhalb, dessen die einzelnen ermittelten Widerstandswerte liegen, wie auch der Mittelwert mit dazugehörigem Konfidenzintervall angegeben. Die Variationen der eigenen Messergebnisse sind dabei nicht als Fehlerangabe zu verstehen, sondern setzen sich aus der Inhomogenität der Probe zusammen. Zur Übersichtlichkeit sind nur Werte aus den aufgelisteten Studien für Defekte und freie Flächen aufgeführt, die auch in dieser Arbeit ermittelt worden sind.

${ }^{5}$ gemittelter Wert für entgegengesetzte Stromrichtungen

${ }^{6}$ Es wird der erhöhte spezifische Flächenwiderstand über dem Übergang von ein- und zweilagigem Graphen angegeben. Dieser beträgt $\rho_{\mathrm{ML} / \mathrm{BL}}=(928 \pm 210) \Omega / \square$. 
114 Ladungstransportmessungen von der atomaren bis zur mesoskopischen Skala auf epitaktischem Graphen

Der von Ji et al. über die Rastertunnelpotentiometrie ermittelte Wert für einlagiges Graphen von (200 \pm 28$) \Omega / \square$ bzw. (227 \pm 36$) \Omega / \square$ für Probenverkippungen von $0,05^{\circ}$ bzw. $0,06^{\circ}$ ist um einen Faktor zwei niedriger [122] als der in dieser Arbeit bestimmte Wert.

Die quantitative Bestimmung der spezifischen Flächen- und Stufenwiderstände erfolgte jedoch nicht anhand der in Kapitel 5.3.1 vorgestellten selbstkonsistenten Modellierung, sondern es wurde angenommen, dass die mikroskopische Stromdichte innerhalb eines Bildbereiches der makroskopischen entspricht. Es wurde mit Hilfe der linearen Regression die mittlere lokale elektrische Feldstärke $\left\langle E_{x}\right\rangle$ bestimmt und der spezifische Flächenwiderstand gemäß $R_{2 d}=\sigma_{2 d}^{-1}=E_{x} / j_{x \text {,makro }}$ berechnet. Für die Berechnung der Widerstände zwischen den einzelnen Übergängen wurden die Spannungsabfälle an den Übergängen bestimmt und die Widerstände gemäß des ohmschen Gesetzes über die makroskopischen Stromdichte ermittelt. Die von Ji et al. in Tabelle 1 aufgelisteten Werte berücksichtigen daher nicht die lokalen Variationen der Stromdichte innerhalb eines Bildausschnittes, welche in der Größenordnung von einem Faktor zwei liegen können, wie in Kapitel 5.3.1 gezeigt worden ist.

Eine weitere Rastertunnelpotentiometrie-Studie von Clark et. al. [130] ermittelt mit Hilfe von Vierpunktmessungen mit Kontaktabständen kleiner als $2 \mu \mathrm{m}$ vergleichbare Flächenwiderstände für ein- und zweilagiges epitaktisches Graphen bei $81 \mathrm{~K}$.

Giannazzo et. al. erhalten auf 4H-SiC an Atmosphäre mit Hilfe des leitfähigen Rasterkraftmikroskops Werte von $(640 \pm 136) \Omega / \square$ für einlagiges Graphen und (313 \pm 60$) \Omega / \square$ für zweilagiges Graphen. Die größere Differenz des spezifischen Flächenwiderstandes zwischen ein- und zweilagigem Graphen im Vergleich zu den eigenen Werten kann daran liegen, dass besonders von einlagigem Graphen die elektronischen Eigenschaften der Oberfläche an Atmosphäre möglicherweise durch Adsorbate sowie einem Wasserfilm beeinflusst werden. Daher ist es denkbar, dass an Atmosphäre durchgeführte Messungen für einlagiges Graphen einen höheren Flächenwiderstand liefern. Des Weiteren besitzt diese Methode eine wesentlich geringere laterale Auflösung von ca. $20 \mathrm{~nm}$. Jobst et. al. erhalten einen nahezu identischen Flächenwiderstand im Vergleich zu dem hier bestimmten von ungefähr $400 \Omega / \square$, welcher bei $25 \mathrm{~K}$ an Hall-Bar-Strukturen von einigen $\mu \mathrm{m}$ Länge und sub $\mu \mathrm{m}$ Breite bestimmt worden ist [138]. 
Aus den ermittelten Flächenwiderständen und der in Kapitel 4.2 bestimmten Ladungsträgerkonzentration von $n \approx 1,3(2) \cdot 10^{13} \mathrm{~cm}^{-2}$ können Transportgrößen wie die mittlere Mobilität $\mu$ oder die mittlere freie Weglänge $l_{\mathrm{mfp}}$ der Ladungsträger für ein- und zweilagiges Graphen abgeschätzt werden. Für die mittlere freie Weglänge $l_{\text {mfp }}$ ergeben sich aus den in Tabelle 1 nach der selbstkonsistenten Modellierung erhaltenen Werten über die Beziehung $l_{\mathrm{mfp}, \mathrm{ML} / \mathrm{BL}}=\sigma_{\mathrm{ML} / \mathrm{BL}} \cdot h /\left(2 \cdot e^{2} \cdot \sqrt{\pi \cdot n}\right)$ Werte von $l_{\mathrm{mfp}, \mathrm{ML}}=40(9) \mathrm{nm}$ und $l_{\mathrm{mfp}, \mathrm{BL}}=$ 39(16) nm. Mit Hilfe der Beziehung $\sigma=\mu \cdot e \cdot n$ erhält man für zweilagiges Graphen eine Mobilität von $\mu_{\mathrm{BL}}=(929 \pm 386) \mathrm{cm}^{2} / \mathrm{Vs}$ und für einlagiges Graphen eine Mobilität von $\mu_{\mathrm{ML}}=$ $(949 \pm 248) \mathrm{cm}^{2} /$ Vs. Dabei wird davon ausgegangen, dass ein- und zweilagiges Graphen eine nahezu identische Elektronenkonzentration $n_{\mathrm{ML}} \approx n_{\mathrm{BL}}$ besitzen, wie es Photoemissionsdaten zeigen [19].

Die alternative Bestimmung der Mobilität über Hall-Messungen an Hall-Bar und Van der Pauw-Strukturen mit Strukturgrößen von $500 \mathrm{~nm}$ bis $5 \mathrm{~mm}$ von ein- bis zweilagigem Graphen bei vergleichbarer Elektronenkonzentration liefern für tiefe Temperaturen (5-25 K) Werte zwischen $1000 \mathrm{~cm}^{2} / \mathrm{Vs}$ und $2000 \mathrm{~cm}^{2} / \mathrm{Vs}$ und stimmen mit den hier rein mikroskopisch mit Hilfe der Rastertunnelpotentiometrie bestimmten Werten gut überein [139, 140]. Bei Raumtemperatur werden im Mittel Werte von $1000 \mathrm{~cm}^{2} /$ Vs erreicht $[138,141]$.

Im Allgemeinen ist die experimentell bestimmte Mobilität von epitaktischem Graphen auf $\mathrm{SiC}(0001)$ wesentlich geringer als die theoretisch erwartete. Für ideales Graphen ohne Defekte erhalten V. Perebeinos et. al. auf Grundlage der semiklassichen Boltzmanntheorie unter Berücksichtigung der Elektronwechselwirkung mit optischen und akustischen Phononen des Graphens sowie der Elektronenwechselwirkung mit polaren Oberflächenphononen des Substrates für Raumtemperatur eine Mobilität von $\mu=81500 \mathrm{~cm}^{2} /$ Vs bei einer Elektronenkonzentration von $5 \cdot 10^{12} \mathrm{~cm}^{2} /$ Vs. Für tiefe Temperaturen ist der Einfluss der Phononen vernachlässigbar und die theoretisch vorhergesagte Mobilität steigt auf Werte von $\mu>10^{6} \mathrm{~cm}^{2} / \mathrm{Vs}$ [142]. Daher wird vermutet, dass der Restwiderstand des epitaktischen Graphens sich aus verschiedenen Streubeiträgen zusammensetzt. Es werden unter anderem die Streuung an Defekten im Interface und dem Substrat, wie positive geladene Störstellen $[140,143]$ und stark lokalisierte Defekte [49], welche z.B. durch fehlende Kohlenstoffatome in der Graphenschicht selbst oder Adatomen auftreten können, diskutiert. 
116 Ladungstransportmessungen von der atomaren bis zur mesoskopischen Skala auf epitaktischem Graphen

Die in Kapitel 3.5.1 identifizierten in der Zwischenschicht liegenden Defekte könnten als geladene Störstellen die Mobilität in den Graphenschichten entsprechend herabsetzten bzw. die mittlere freie Weglänge erhöhen und damit für den hohen Restwiderstand verantwortlich sein.

Ebenso spielt bei den epitaktisch gewachsenen Graphen-Proben die Dichte, Anzahl und Art der auftretenden Stufen und charakterisierten Übergänge bei makroskopischen Transportuntersuchungen eine entscheidende Rolle. So ist der Spannungsabfall auf der freien Fläche von einlagigem Graphen auf einer Länge von $85 \mathrm{~nm}$ identisch zu dem an einem ML-BLÜbergang des Typs I. Der Einfluss der Falten bzw. Wrinkles sowie den auftretenden Punktdefekten, wie sie in Kapitel 3.5.3 identifiziert worden sind, kann anhand der lokalen Transportuntersuchungen nicht eindeutig bestimmt werden, da ein Spannungsabfall an diesen Strukturen nur schwer zu bestimmen ist.

Die Rastertunnelpotentiometrie-Studie von Clark et. al. [130] erhält sehr niedrige Werte für einen untersuchten ML-BL-Übergang sowie einem ML-ML-Übergang mit jeweils darunterliegender Substratstufe. Jedoch ist hier anzumerken, dass überraschenderweise auf den freien Flächen vor- und hinter den Übergängen kein Gradient im elektrochemischen Potential zu erkennen ist.

Ji et al. fanden einen linearen Zusammenhang zwischen dem spezifischen Stufenkantenwiderstand von Übergängen einlagigen Graphens und deren Stufenhöhe. So beträgt der Stufenkantenwiderstand von ML-ML-Stufen der Höhe $0,5 \mathrm{~nm}(6,9 \pm 2,9) \Omega \mu \mathrm{m}$, für $1 \mathrm{~nm}$ hohe Stufen $(14,9 \pm 3,6) \Omega \mu \mathrm{m},(24,7 \pm 4,3) \Omega \mu \mathrm{m}$ für 1,5 nm Stufenhöhe [122]. Für Übergänge zwischen ein- und zweilagigem Graphen wurden etwas höhere Werte von $(20,9 \pm 5,7) \Omega \mu \mathrm{m}$ für ML-BL-Übergänge des Typs II und $(28 \pm 4,7) \Omega \mu \mathrm{m}$ für ML-BL-Übergänge, bei denen vermutlich drei Substratstufen unter dem einlagigem Graphen liegen bestimmt. Nach Angabe der Autoren ist die Anzahl der an verschiedenen Positionen der Probe untersuchten Übergänge bzw. Stufen vergleichbar zu denen in dieser Arbeit. Die Streuung der erhaltenen Widerstandswerte für den ML-BL-Übergang des Typs II ist etwas niedriger als die in dieser Arbeit bestimmten Widerstandswerte. Daten zu einer quantitativen Auswertung anhand einer selbstkonsistenten Modellierung wurde nicht publiziert, ein relevanter Unterschied im Ergebnis hingegen ausgeschlossen. 
Eine Übereinstimmung mit den eigenen Daten findet sich in der Tendenz, dass der spezifische Widerstand von ML-ML-Übergängen mit der Anzahl der darunterliegenden Substratstufen wächst und dass im Mittel die Flächenwiderstände von ein- und zweilagigem Graphen sich nicht signifikant unterscheiden. Der Widerstand von ML-BL-Übergänge des Typ I und II ist nach beiden Auswertmethoden höher als der des ML-ML-Übergangs des Typs I. Da ML-BL-Übergänge des Typs I und II eine vergleichbare bzw. niedrigere Stufenhöhe von 330 pm bzw. 70 pm wie ML-ML-Übergänge des Typs I (250 pm) besitzen, ist davon auszugehen, dass sich die mikroskopischen Streumechanismen in ML-BL-Übergängen im Vergleich zu ML-ML-Übergängen unterscheiden.

Für die Ursache des erhöhten Widerstandes an ML-ML-Übergängen und ML-BLÜbergängen werden verschiedene physikalische Ursachen diskutiert. Auf die einzelnen Theorien soll im Zusammenhang mit höchstauflösenden Messungen des Verlaufes des elektrochemischen Potentials in der Nähe von ML-BL-Übergängen und ML-MLÜbergängen im nächsten Kapitel eingegangen werden.

\subsection{Verlauf des elektrochemischen Potentials in der Umgebung von lokalisierten Defekten}

Bisher wurde der räumliche Verlauf des elektrochemischen Potentials auf größeren Längenskalen analysiert und versucht diesen mit dem Modell des klassischen diffusiven Ladungstransportes zu beschreiben. Größte Änderungen des elektrochemischen Potentials sind an lokalisierten Defekten wie den ML-BL und ML-ML-Übergängen beobachtet worden. Im Folgenden soll der Verlauf des elektrochemischen Potentials an diesen Defekten innerhalb der Fermiwellenlänge $\left(\lambda_{\mathrm{F}} \approx 10 \mathrm{~nm}\right)$ vor und hinter einem Übergang näher untersucht werden. Im einfachsten Fall einer quantenmechanischen Barriere sollte der Spannungsabfall symmetrisch um den Ort der Barriere abfallen und die räumliche Ausdehnung stark lokalisiert sein [29]. Die Ausdehnung des Potentialsprungs im elektrochemischen Potential hängt vermutlich von zwei Faktoren ab. Zum einen wird der Verlauf durch die Breite und Höhe des Streupotentials beeinflusst werden. Zum anderen wird die Ankopplung von

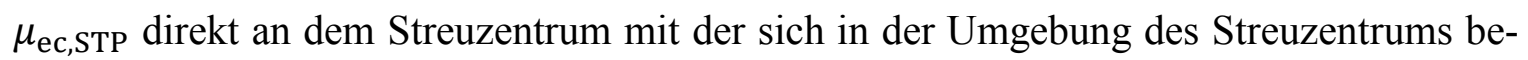
findlichen Potentialverteilung eine Rolle spielen. Erste Untersuchungen diesbezüglich wurden an monoatomaren Stufen der Si(111)- $\sqrt{3} \times \sqrt{ } 3-A g$ Rekonstruktion mit Hilfe des STP- 
118 Ladungstransportmessungen von der atomaren bis zur mesoskopischen Skala auf epitaktischem Graphen

Verfahrens durchgeführt [132]. Es konnte gezeigt werden, dass $\mu_{\mathrm{ec}, \mathrm{STP}}$ eine endliche Übergangsweite von $0,5 \mathrm{~nm}$ hat und der eigentliche Potentialsprung um $\lambda_{\mathrm{F}} / 4$ vor der monoatomaren Stufe lokalisiert ist.

Als physikalische Ursache für den erhöhten Widerstand an ML-BL-Übergängen und MLML-Übergängen werden verschiedene Effekte, die zur Streuung der Elektronen führen, diskutiert. Am Übergang von einlagigem Graphen wird ein durch die Substratstufe hervorgerufenes Abheben der oberen Graphenlage verursachtes endliches Streupotential vermutet [144]. Bei ML-BL-Übergängen geht man von einer schlechten Kopplung der Wellenfunktionen der einzelnen Lagen am Interface als Hauptursache für den Widerstand aus [122, 134]. Diese beiden Theorien sollen im Zusammenhang mit dem hier bestimmten Verlauf des lokalen elektrochemischen Potentials in der Umgebung von ML-BL- und ML-MLÜbergängen diskutiert werden. Der atomar aufgelöste Verlauf des elektrochemischen Potentials an einem ML-BL-Übergangs des Typs I ist in Abbildung 60 mit einer Diskretisierungsweite von $0,1 \mathrm{~nm}$ aufgenommen worden. In der Topographie von Abbildung 60 (a) ist auf der linken Seite einlagiges und auf der rechten Seite zweilagiges Graphen zu erkennen. Die obere Graphenschicht am Übergang zwischen dem ein- und zweilagigem Graphen ist durchgängig, die Topographie zeigt im Bereich des Überganges eine leichte Überhöhung bzw. Wölbung von 100 pm, bevor die mittlere Höhe der zweilagigen Graphenschicht erreicht wird. Dabei kann es sich wie bei den in Kapitel 3.5.3 gezeigten Wrinkles um eine Faltung der oberen, durchgängigen Graphenschicht handeln. Der Verlauf von $\mu_{\mathrm{ec}, \mathrm{STP}}$ ist in Abbildung 60 (b) zu erkennen. Betrachtet man innerhalb der Fermiwellenlänge vor und hinter dem Streuzentrum das elektrochemische Potential, beobachtet man vor und hinter dem Übergangsbereich ein nahezu konstantes Potential und am Übergang selbst einen auf wenige Nanometer lokalisierten Anstieg. 
a)

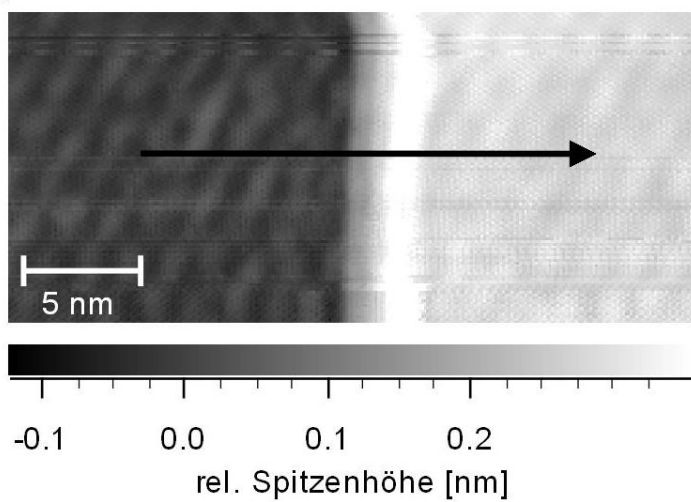

b)

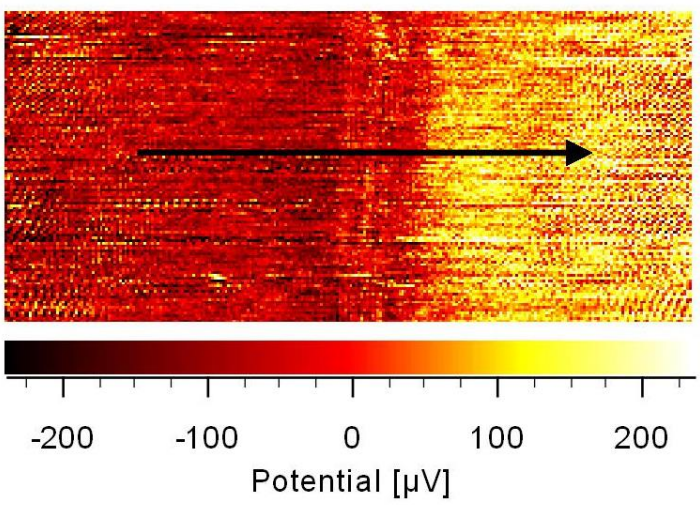

c)

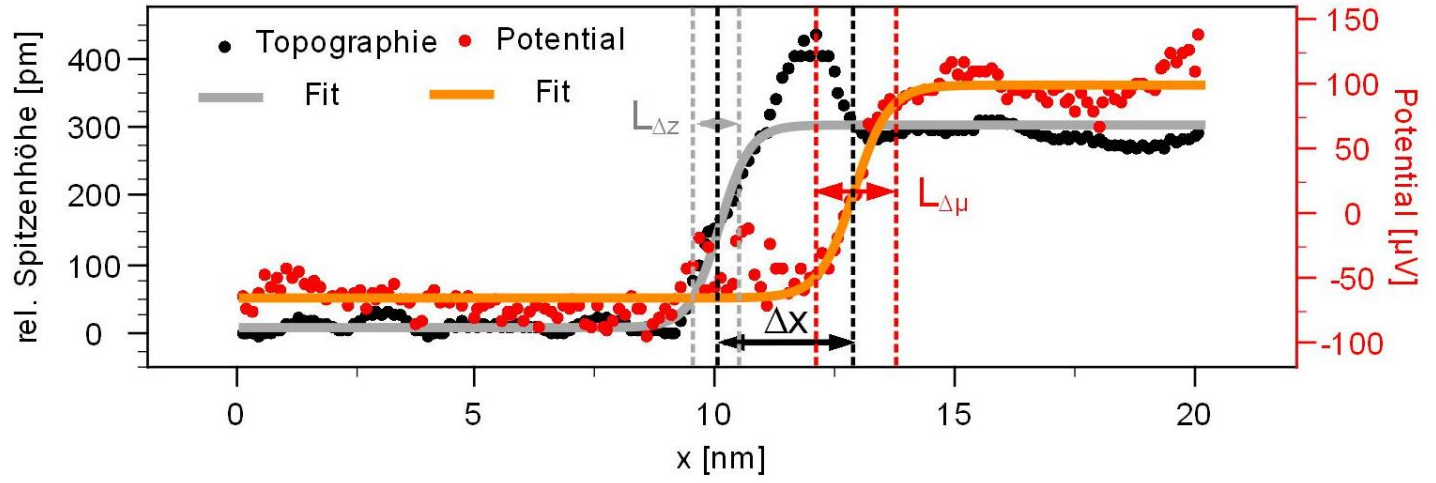

d)
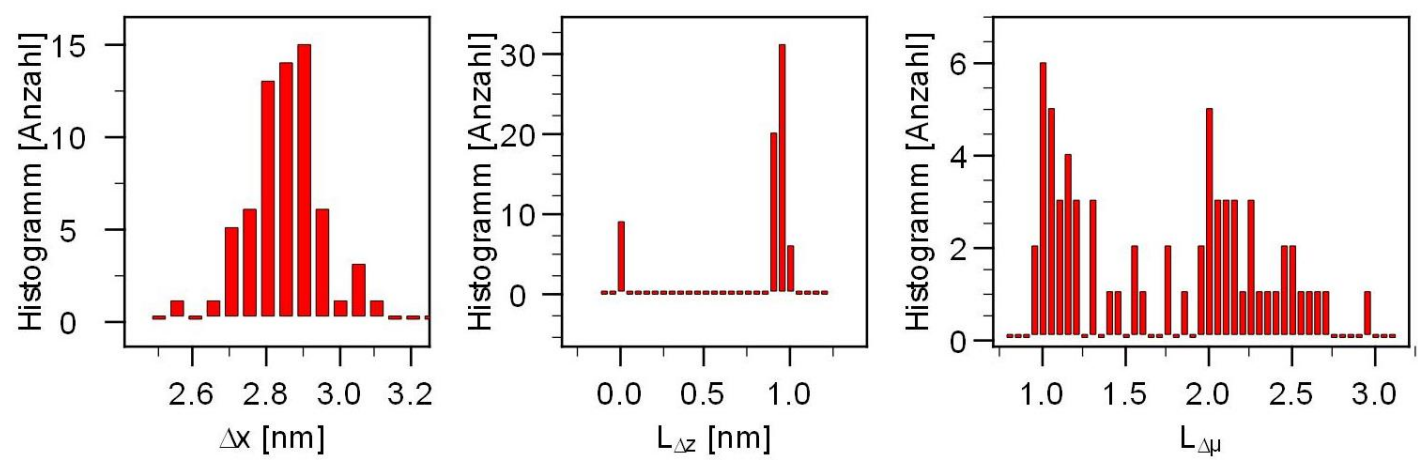

Abbildung 60: (a) Topographie eines ML-BL-Übergangs des Typs I und (b) aufgenommenes thermospannungsbereinigtes lokales Potential $\left(I_{\mathrm{T}}=240 \mathrm{pA}, U_{\mathrm{Bias}}=-40 \mathrm{mV}, j_{\text {makro }}=3,8 \mathrm{~A} / \mathrm{m}, 19 \times 14 \mathrm{~nm}^{2}, 253 \times 116\right.$ Pixel $)$. (c) Schnitt entlang des in (a) und (b) eingezeichneten Bereiches. Der Spannungsabfall an dem ML-BLÜbergang zeigt einen lateralen Versatz $\Delta \mathrm{x}$ im Vergleich zum Übergang zwischen ein- und zweilagigem Graphen. Sowohl der topographische Übergang als auch der Spannungsabfall sind stark lokalisiert. ML-BLÜbergang als auch der Potentialsprung werden jeweils durch eine angepasste Stufenfunktion beschrieben. (d) Histogramme des zeilenweise bestimmten lateralen Versatzes $\Delta \mathrm{x}$, lateralen Ausdehnung der Topographie $L_{\Delta \mathrm{z}}$ sowie Ausdehnung $L_{\Delta \mu}$ des Sprungs im elektrochemischen Potential anhand angepasster Stufenfunktionen. 
120 Ladungstransportmessungen von der atomaren bis zur mesoskopischen Skala auf epitaktischem Graphen

Die laterale Position der Stufe und des Potentialsprungs fallen dabei nicht zusammen, wenn man die Position der Stufe bzw. des Potentialsprungs bei jeweils $50 \%$ ihres Anstieges definiert.

Um den Verlauf des Potentials im Bezug zum Übergang in der Topographie näher zu untersuchen, ist ein lateraler Schnitt der Topographiedaten als auch Potentiometriedaten in Abbildung 60 (c) dargestellt. Der Verlauf der Stufe $h(x)$ als auch des elektrochemischen Potentials $\mu_{\mathrm{ec}, \mathrm{STP}}(x)$ lässt sich in erster Näherung gut durch eine nummerisch angepasste Stufenfunktion der Form

$$
\begin{gathered}
\mu_{\mathrm{ec}, \mathrm{STP}}(x)=\Delta \mu_{\mathrm{ec}} \cdot\left(1+e^{\frac{x-x_{0}}{L_{\Delta \mu}}}\right)^{-1}+\Delta \mu_{\mathrm{Offset}} \text { bzW. } \\
h(x)=\Delta z \cdot\left(1+e^{\frac{x-x_{0}}{L_{\Delta z}}}\right)^{-1}+\Delta z_{\mathrm{Offset}}
\end{gathered}
$$

beschreiben. Dabei legt $x_{0}$ die absolute Position, $\Delta \mu_{\mathrm{ec}}$ bzw. $\Delta z$ die jeweiligen Amplituden und $L_{\Delta \mu}$ und $L_{\Delta z}$ die laterale Ausdehnung der Topographie sowie des elektrochemischen Potentialsprunges fest. Aufgrund der Variationen des Messsignals und zur Erhöhung der Auflösung wird in Abbildung 60 zuvor ein Boxfilter, welcher jeweils über 6 Zeilen den Mittelwert bestimmt, verwendet. In Abbildung 60 (d) ist die Verteilung der erhaltenen Größen für den lateralen Versatz zwischen Topographie- und Potentialsprung sowie deren laterale Ausdehnung dargestellt. Als Mittelwert erhält man für $\Delta x=2,8(5) \mathrm{nm}, L_{\Delta \mu}=$ $1,7(6) \mathrm{nm}$ und $L_{\Delta z}=0,8(4) \mathrm{nm}$. Als Variation der bestimmten Größen ist die Standardabweichung angegeben.

Zum Vergleich ist in Abbildung 61 (a) und (b) der Verlauf der Topographie sowie von $\mu_{\text {ec,STP }}$ an einem ML-ML-Übergang mit zwei darunter liegenden Substratstufen dargestellt. Um die Daten analog zu den in Abbildung 60 vorgestellten Daten auszuwerten, wurden alle Zeilen in $\mathrm{x}$-Richtung derart verschoben, dass die Stufe senkrecht entlang der y-Achse ausgerichtet ist. Dieselbe Operation wird mit den Potentialwerten durchgeführt. Abbildung 61 (c) zeigt einen Ausschnitt der transformierten Topographie- und Potentialdaten aus Abbildung 61 (a) und (b). Diese Ausrichtung der Daten erlaubt eine Mittelung der Potentialwerte entlang der vertikalen Richtung. Trotz der im Vergleich zu Abbildung 60 geringeren Auflö- 
a)

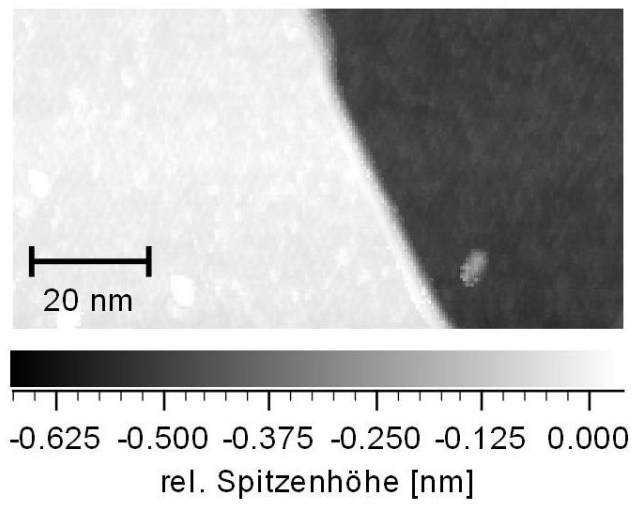

c)
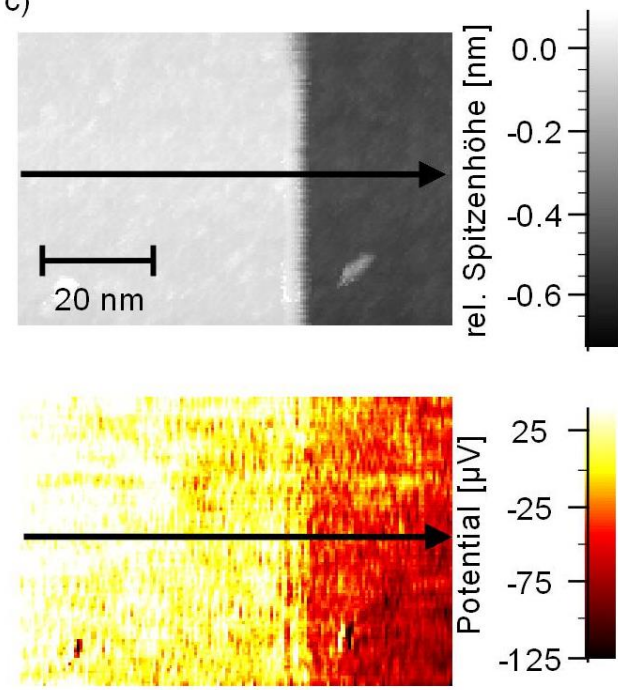

b)

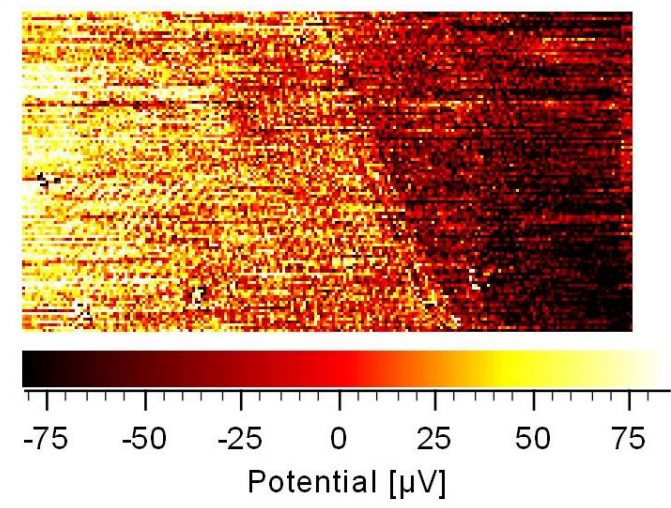

d)
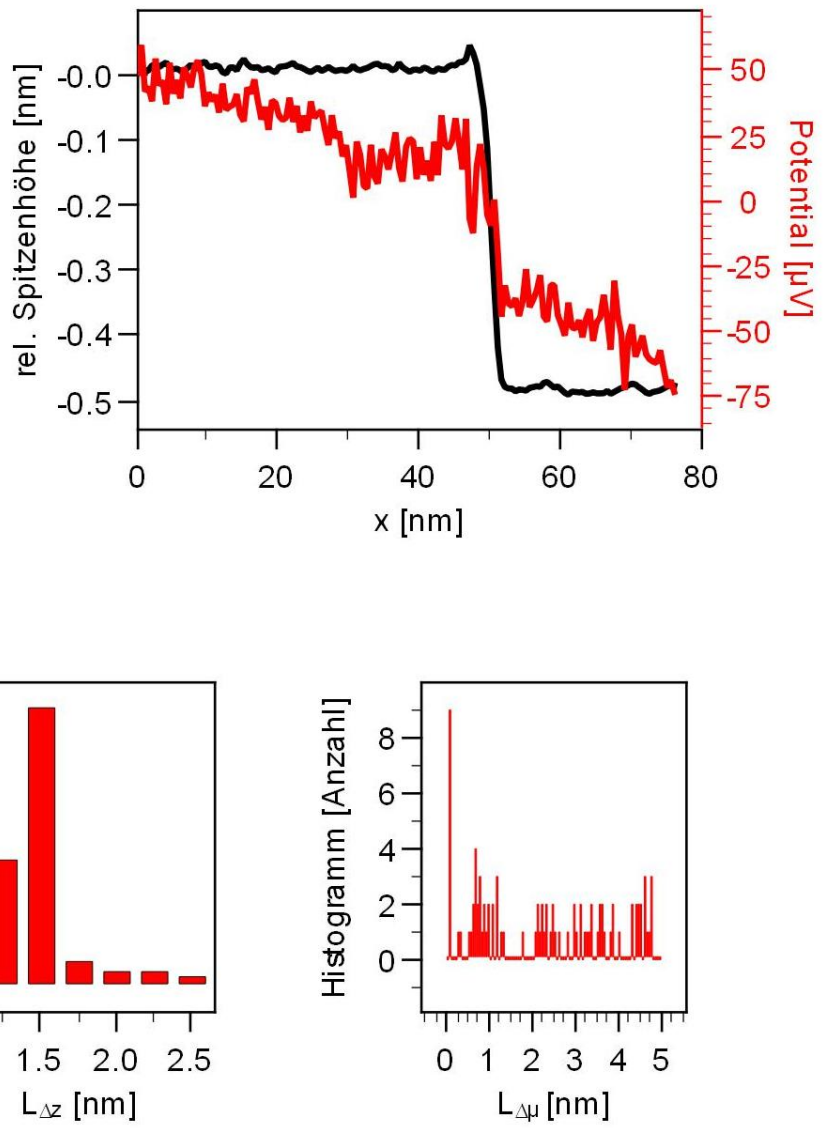

Abbildung 61: (a, b)Topographie und Probenpotential an einem ML-ML-Übergang mit zwei darunter-liegenden Substratstufen $\left(104 \times 56 \mathrm{~nm}^{2}, U_{\mathrm{Bias}}=70 \mathrm{mV}, I_{\mathrm{T}}=1 \mathrm{nA}\right)$. (c) Linienweise ausgerichtete Topographie- und Potentialwerte in der Umgebung des Übergangs. (d) Über $10 \mathrm{~nm}$ entlang der vertikalen Rasterrichtung gemittelte Topographie- sowie Potentiometrie-Daten. (e) Histogramme des zeilenweise bestimmten lateralen Versatz $\Delta x$, lateralen Ausdehnung der Topographie $L_{\Delta z}$ sowie Ausdehnung $L_{\Delta \mu}$ des Sprunges im elektrochemischen Potential anhand angepasster Stufenfunktionen. 
122 Ladungstransportmessungen von der atomaren bis zur mesoskopischen Skala auf epitaktischem Graphen

sung von 0,5 nm ist anhand des Schnittes in Abbildung 61 (e) zu erkennen, dass der Sprung in $\mu_{\text {ec,STP }}$ im Gegensatz zu dem in Abbildung 60 gezeigten ML-BL-Übergang direkt an der Stufe lokalisiert ist. Als Mittelwert erhält man aus den Abbildung 61 (e) dargestellten Histogrammen $\Delta x=(0,02 \pm 1) \mathrm{nm}, L_{\Delta \mu}=(2,4 \pm 1,7) \mathrm{nm}$ und $L_{\Delta z}=(1,5 \pm 0,3) \mathrm{nm}$.

Zur systematischen Untersuchung des Verlaufes des elektrochemischen Potentials an lokalisierten Defekten wurden 13 ML-BL-Übergänge des Typs I und II sowie drei ML-MLÜbergange mit einer Stufenhöhe von 250 pm bzw. 500 pm untersucht. Die laterale Auflösung der Daten ist bei allen untersuchten Datensätzen $\leq 0,5 \mathrm{~nm}$. Folgende Tabelle fasst die Ergebnisse zusammen:

\begin{tabular}{|c|c|c|c|}
\hline & $\Delta x[\mathrm{~nm}]$ & $L_{\Delta \mu}[\mathrm{nm}]$ & $L_{\Delta z}[\mathrm{~nm}]$ \\
\hline $\begin{array}{c}\text { ML-BL-Übergang } \\
\text { Typ I \& II }\end{array}$ & $2,0(5)$ & $4,0 \pm 1,3$ & $0,5(4)$ \\
\hline $\begin{array}{c}\text { ML-ML-Übergang } \\
\text { Typ I \& II }\end{array}$ & $0,3(4)$ & $2 \pm 1,7$ & $0,9(5)$ \\
\hline
\end{tabular}

Die Potentialsprünge an einem ML-BL-Übergänge zeigen im Mittel einen lateralen Versatz des Potentialsprungs von $\Delta x=2,0(5) \mathrm{nm}$ und sind auf dem zweilagigen Graphen lokalisiert. Im Vergleich zur Übergangsweite $L_{\Delta z}$ in der Topographie ist die laterale Ausdehnung des Potentialsprungs größer und beträgt $L_{\Delta \mu}=(4,0 \pm 1,3) \mathrm{nm}$.

Die ML-ML-Übergänge zeigen hingegen keinen signifikanten Versatz zwischen dem topographischen Übergang und dem Sprung im elektrochemischen Potential. Die unterschiedliche räumliche Lokalisierung und Ausdehnung von $\mu_{\mathrm{ec}, \mathrm{STP}}$ ist neben dem höheren Widerstand für ML-BL-Übergänge im Vergleich zu ML-ML-Übergängen ein starkes Indiz für unterschiedliche Streumechanismen an den verschiedenen Defekttypen, wie im Folgenden erläutert werden soll.

\section{ML-ML-Übergang}

Um die Streueigenschaften an ML-ML-Übergängen zu untersuchen, wurde von Low et. al. zum einen der Einfluss der Krümmung und Verspannung innerhalb der Ebene von bis zu $1 \%$ und zum anderen die elektrische Kopplung der Graphenlage zum darunterliegenden SiC-Substrat untersucht. Der Einfluss der Krümmung wird für Stufen mit einer bzw. zwei 
darunterliegenden Substratstufen mit $R<0,01 \Omega \mu \mathrm{m}$ angegeben, und der Beitrag durch die Verspannung der Graphenschicht in der Ebene mit $R<1 \Omega \mu \mathrm{m}$ abgeschätzt. Der wesentliche Beitrag zum Stufenwiderstand kommt nach Low et al. durch eine vom Abstand der Zwischenschicht und der oberen Graphenschicht abhängigen Dotierung zustande. Die lineare Abhängigkeit des Widerstandes von der Stufenhöhe von ML-ML-Übergängen kann anhand theoretischer Berechnungen von Low et al. gut beschrieben werden und zeigt mit den experimentellen Daten von Ji et al. eine gute Übereinstimmung [144]. In Abbildung 62 ist der von Low et al. berechnete Verlauf der ersten Graphenlage relativ zur darunterliegenden Zwischenschicht an Stufenkanten unterschiedlicher Höhe dargestellt.

a)

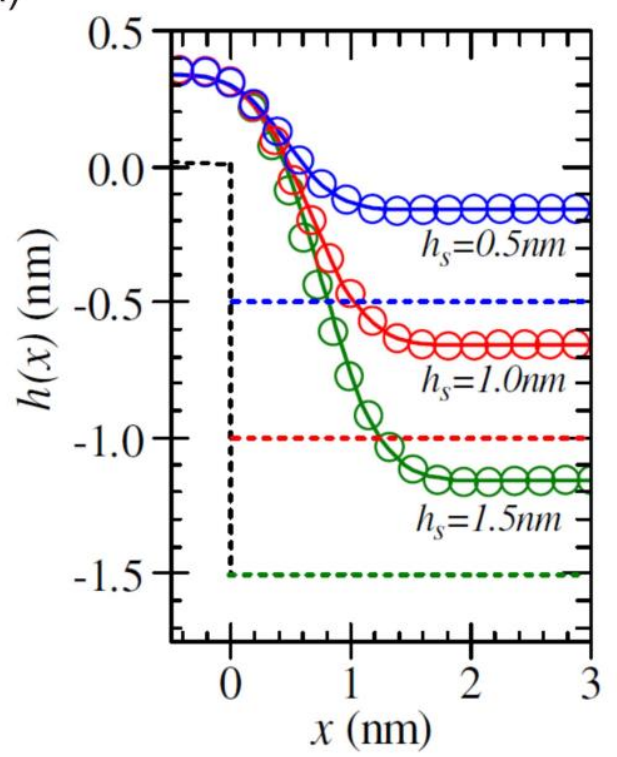

b)

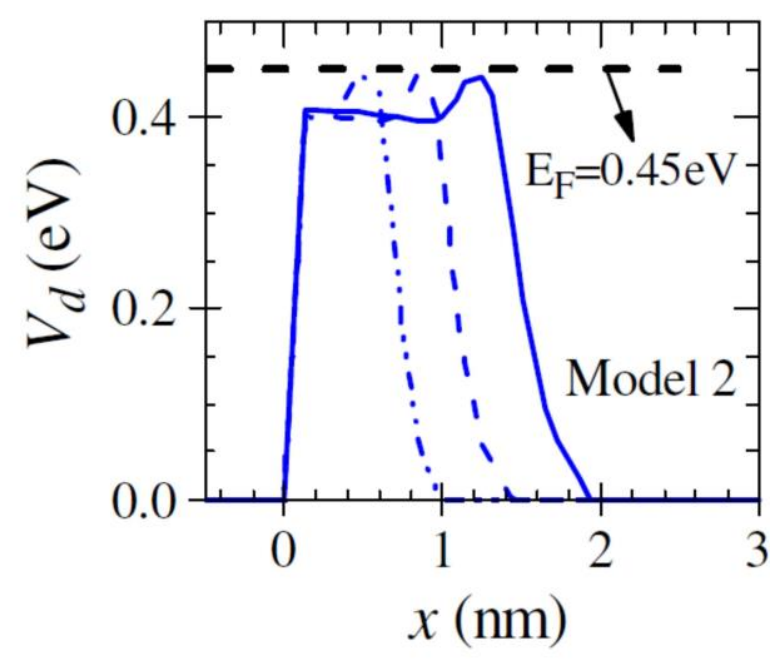

Abbildung 62: (a) Theoretisch berechneter Verlauf der oberen Graphenschicht für drei unterschiedliche Stufenhöhen von ML-ML-Übergängen und (b) berechnetes Streupotential für die entsprechenden Stufenhöhen nach [144]. Das Streupotential ist nahezu kastenförmig, die Breite der Potentialbarriere nimmt näherungsweise linear mit ansteigender Stufenhöhe zu und kann als symmetrisch um den Kantenverlauf der Graphenlage angenommen werden.

Während im Gleichgewichtsfall der Abstand zur Zwischenschicht 2,6 nm beträgt, löst sich an der Stufe die erste Graphenlage von der Zwischenschicht ab, so dass sich der Abstand zur darunterliegenden Lage vergrößert. Während einlagiges Graphen auf epitaktischem Graphen im Gleichgewichtsabstand auf der freien Fläche stark n-dotiert ist ( $E_{\mathrm{F}} \approx 0,44 \mathrm{eV}$ ), nimmt mit zunehmendem Abstand der Graphenlage die Kapazität und damit die in die obere Graphenschicht induzierte Ladungsdichte ab. Nach diesem Modell ergibt sich ein 
124 Ladungstransportmessungen von der atomaren bis zur mesoskopischen Skala auf epitaktischem Graphen

wie in (b) gezeigtes kastenförmiges vor der eigentlichen Substratstufe lokalisiertes Streupotential, welches abhängig von der Stufenhöhe breiter wird. Für die niedrigste Stufenhöhe ergibt sich eine Ausdehnung des Streupotentials von ca. $1 \mathrm{~nm}$.

Demnach ist nach dieser Theorie und den postulierten Eigenschaften des elektrochemischen Potentials ein symmetrisch um dieses Streupotential lokalisierter Potentialsprung im elektrochemischen Potential zu erwarten. Sowohl die laterale Position des experimentell bestimmten Potentialsprungs in Bezug auf die Topographie der ML-ML-Übergänge $\Delta x_{\mathrm{ML}-\mathrm{ML}}$ wie auch die bestimmte mittlere Ausdehnung von $L_{\Delta \mu, \mathrm{ML}-\mathrm{ML}}$ stimmen sehr gut mit dieser Modellvorstellung überein.

\section{ML-BL-Übergang}

Um den dominierenden Streumechanismus an ML-BL-Übergängen zu verstehen, wurden Quantentransport-Berechnungen an gezackten und gestaffelten BL-ML-BL-Übergängen von Giannazzo et. al durchgeführt [134]. Abbildung 63 zeigt schematisch die zur Berechnung verwendete Struktur, die zwei gezackte ML-BL-Übergänge enthält (BL-ML-BLStruktur), die durch einlagiges Graphen verbunden sind. Der berechnete Leitwert der in (a) gezeigten Struktur ist in Abbildung 63 (b) dargestellt. Das Ergebnis zeigt einen starken Einbruch der Leitfähigkeit im Energieintervall von $\pm 0,48 \mathrm{eV}$ um den Dirac-Punkt für die gezackte BL-ML-BL-Struktur im Vergleich zu einlagigem Graphen. Für die gestaffelte BL-ML-BL-Struktur wird für das Valenzband $\left(E-E_{\mathrm{F}}<0\right)$ ein vergleichbarer Einbruch des Leitwertes beobachtet, während für das Leitungsband der Streumechanismus nicht so stark zu dominieren scheint. Der Streumechanismus wird durch eine schwache Kopplung der Wellenfunktionen des $\pi$ bzw. $\pi^{*}$-Bandes des einlagigen Graphens und der Wellenfunktionen der ersten beiden Bänder des zweilagigen Graphens verursacht. Abbildung 63 (c) zeigt die zugehörige berechnete Transmission über die gesamte Struktur aus (a) sowie im Inset für ein- und zweilagiges Graphen. Nach Giannazzo et. al ändert der Streumechanismus sowohl für gezackte als auch gestaffelte ML-BL-Übergänge seine Eigenschaften nicht, wenn bei ansonsten gleichbleibender Geometrie der Bereich des einlagigen Graphens und damit der Abstand der beiden ML-BL-Übergänge auf unter 2,8 nm verkürzt wird. 
(a) $d_{1 L}$

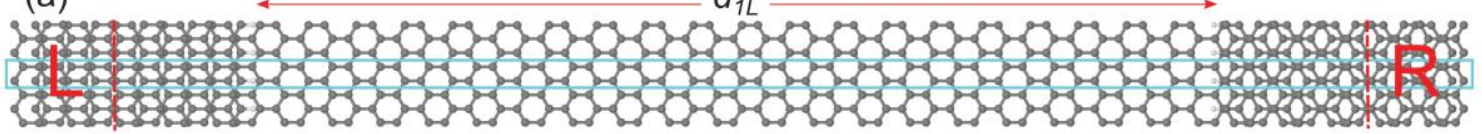

(b)
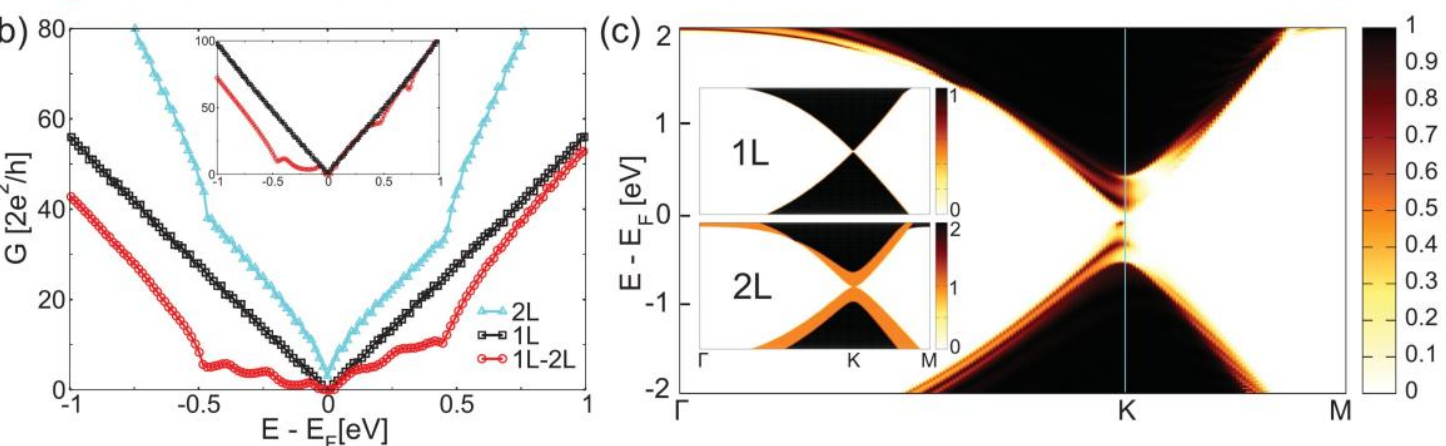

Abbildung 63: Simulation der Transporteigenschaften eines BL-ML-BL-Übergangs nach Giannazzo et al. [134] (a) Schematische Darstellung der simulierten Struktur. An den Kontakten links und rechts befindet sich zweilagiges Graphen, in der Mitte einlagiges Graphen der Länge $d_{1 L}$. Die ML-BL-Übergänge sind gezackt. (b) Berechneter Leitwert $G$ in Abhängigkeit von der Energie im Intervall $\pm 1 \mathrm{eV}$ für eine Simulationsstruktur aus einlagigem Graphen (schwarz, 1L), zweilagigem Graphen (blau, 2L) und einem wie in (a) dargestellten gezacktem BL-ML-BL-Übergang (rot, 1L-2L). Der Inset zeigt den Leitwert einlagigen Graphens (schwarz) im Vergleich zu einem gestaffeltem BL-ML-BL-Übergang.

Daraus kann geschlossen werden, dass der Streumechanismus stark lokalisiert ist und entsprechend sowohl für gezackte als auch gestaffelte ML-BL-Übergänge auf der Seite von einlagigem Graphen räumlich auf einen Bereich unterhalb von 1,4 nm eingeschränkt sein muss.

Die hier experimentell gefundene Variation von $L_{\Delta \mu} \approx 4 \mathrm{~nm}$ und gleichzeitige Lokalisierung auf der oberen Terrasse ist mit diesem Modell zunächst vereinbar. Jedoch wird von Giannazzo et al. unter anderem nicht das Abheben der Monolage an dem Übergang berücksichtigt. Betrachtet man nochmals Abbildung 60 (c), erkennt man, dass das elektrochemische Potential auch schon am Ort der Stufenkante einen leichten Anstieg zeigt. Daher ist im Rahmen der genannten Modelle davon auszugehen, dass an ML-BL-Übergängen beide Streumechanismen berücksichtigt werden müssen. Die Transmission der Stufe sollte durch das Abheben der oberen Graphenlage und durch die schlechte Ankopplung der Wellenfunktionen zwischen ein- und zweilagigem Graphen herabgesetzt werden. Die für die beiden Streumechanismen räumlichen Bereiche müssen jedoch nicht zwingend zusammenfallen, so dass, wie auch die experimentellen Daten zeigen, die Übergangsweite im elektrochemi- 
126 Ladungstransportmessungen von der atomaren bis zur mesoskopischen Skala auf epitaktischem Graphen

schen Potential $L_{\Delta \mu, \mathrm{ML}-\mathrm{BL}}$ tendenziell größer als bei einem ML-ML-Übergang ist. Eine Erklärung für den lateralen Versatz des Potentialsprunges an einem ML-BL-Übergang auf die Seite des zweilagigen Graphen liefert die Theorie nicht. Es ist jedoch festzuhalten, dass wenn auf Längenskalen unterhalb der Fermiwellenlänge der Abfall im lokalen elektrochemischen Potential mit dem Ort des Streupotentials übereinstimmt, der größte Anteil des Streupotentials erst auf der oberen Terrasse lokalisiert zu sein scheint. Der laterale Versatz des Potentialsprungs hin zum zweilagigen Graphen von im Mittel 2 nm kann im Vergleich zu einlagigem Graphen z.B. dadurch erklärt werden, dass die ungestörten elektronischen Eigenschaften des zweilagigen Graphen erst auf der oberen Terrasse nach der beschriebenen topographischen Überhöhung einsetzten. Dabei ist es denkbar, dass unter der abgehobenen oberen Graphenlage im Bereich von $2 \mathrm{~nm}$ keine weitere Graphenlage darunterliegt.

Abbildung 64 zeigt Messungen der räumlich aufgelösten differentiellen Leitfähigkeit im Energiebereich von -130 mV bis $130 \mathrm{mV}$ an einem ML-BL-Übergang des Typs I. Abbildung 64 (a) ist die Topographie und in Abbildung 64 (b) ein lateraler Schnitt entlang des ML-BL-Überganges dargestellt. Der topographische Verlauf des ML-BL-Übergangs weist ebenso wie in Abbildung 60 eine Überhöhung der oberen Graphenlage am Übergang auf. In Abbildung 64 (c) ist die differentielle Leitfähigkeit bei $\mathrm{E}_{\mathrm{F}}$ dargestellt. Im direkten Vergleich zwischen dem Bereich mit einer einlagigen Graphen- und zweilagigen Graphen-Bedeckung erkennt man Unterschiede im dI/dU-Signal, welches in erster Näherung proportional zur lokalen Zustandsdichte ist. Mit der topographischen Überhöhung ist ebenso eine Erhöhung der differentiellen Leitfähigkeit über die Werte des ein- und zweilagigen Graphens sichtbar. In Abbildung 64 (d) ist die differentielle Leitfähigkeit im Energiebereich von -130 bis $+130 \mathrm{mV}$ entlang des in Abbildung 64 (a) durch einen grauen Pfeil gekennzeichneten und in Abbildung 64 (b) dargestellten Bereiches aufgetragen. Die größte Änderung der differentiellen Leitfähigkeit am Übergang erstreckt sich über 1-2 nm am Ort der topographischen Überhöhung der oberen Graphen-Schicht. Auf dem ein- und zweilagigem Graphen sind zwar ebenso Modulationen erkennbar, diese fallen jedoch nicht so stark wie direkt am Übergang aus. 
a)

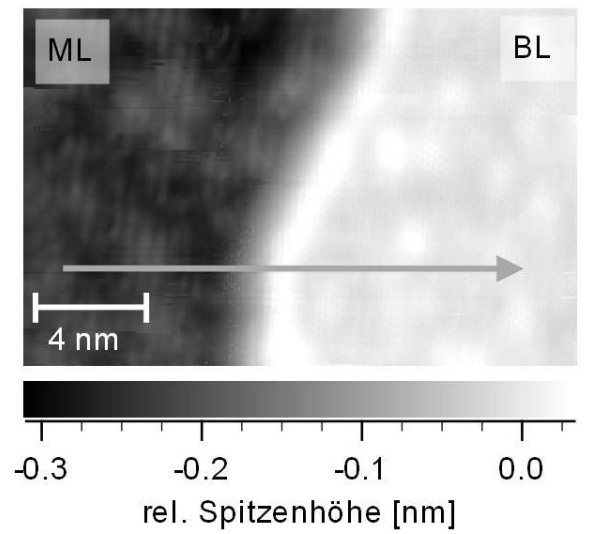

c)

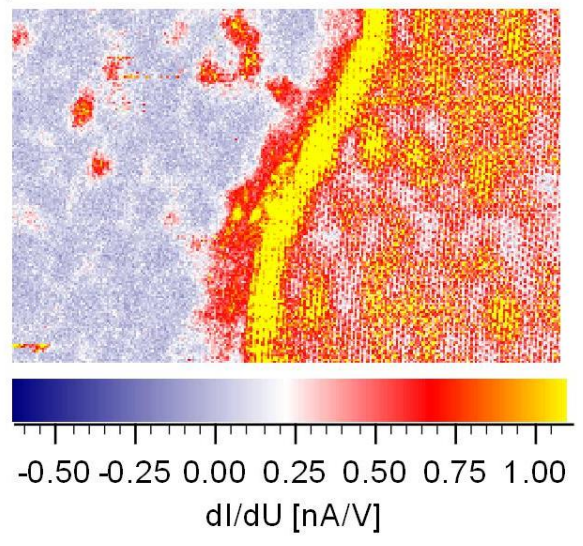

b)

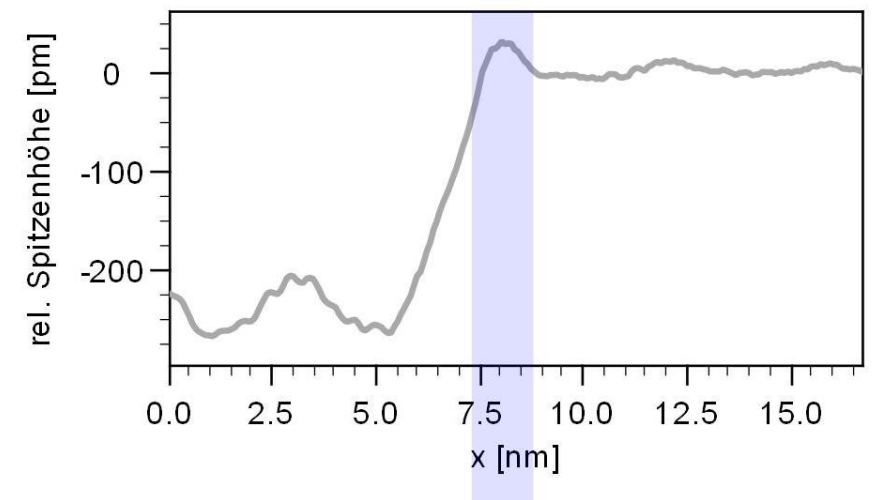

d)

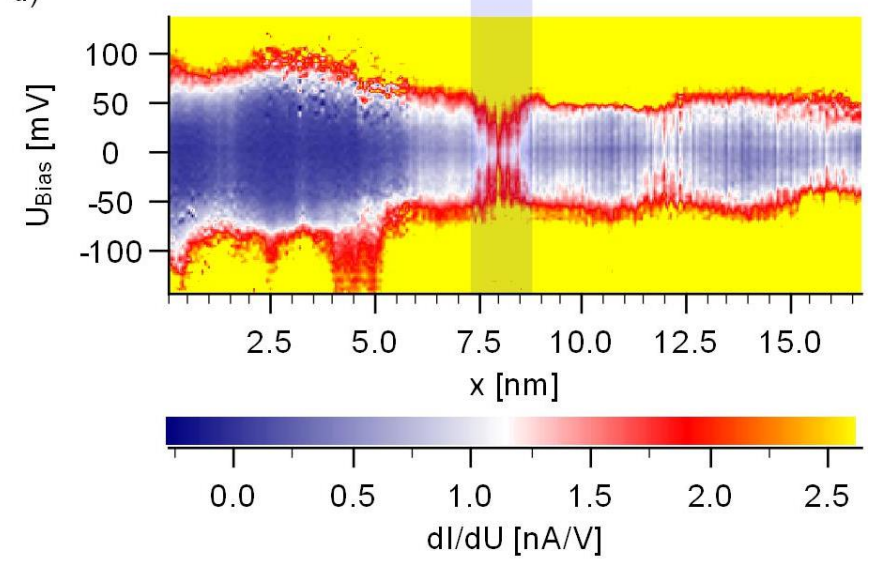

Abbildung 64: Direkter Vergleich der elektronischen Eigenschaften von ein- und zweilagigem Graphen in der unmittelbaren Umgebung eines ML-BL-Übergangs (Typ I). (a) Topographie und (b) horizontaler Schnitt entlang des ML-BL-Übergangs. (c) Signal der differentiellen Leitfähigkeit bei E $E_{F}$ zeigt verschiedene elektronische Kontraste für einlagiges und zweilagiges Graphen sowie am Übergang im Bereich des ,gefalteten“ Graphens ein erhöhtes dI/dU-Signal. (d) räumlich aufgelöstes dI/dU-Signal im Spannungsbereich von -130 $\mathrm{mV}$ bis $+130 \mathrm{mV}$ entlang des horizontalen Schnittes aus (a) bzw. (b). Neben der Änderung der differentiellen Leitfähigkeit zwischen ein- und zweilagigem Graphen ist ebenso ein mit dem ,gefalteten“ Graphenbereich korrelierte Änderung der differentiellen Leitfähigkeit erkennbar (blau schraffierter Bereich).

Zusammenfassend lässt sich festhalten, dass die elektronischen Eigenschaften am ML-BLÜbergang im Bereich des beschriebenen topographischen Überganges im Vergleich zu einund zweilagigem Graphen gestört sind und sich die Störung über die Ausdehnung der Stufenkante $L_{\Delta z}$ und zusätzlich über die Überhöhung bzw. Wölbung der oberen Graphenlage erstreckt. Dieses Ergebnis deckt sich mit der Beobachtung der lateralen Ausdehnung des Potentialsprungs von $L_{\Delta \mu} \approx 4 \mathrm{~nm}$. 
128 Ladungstransportmessungen von der atomaren bis zur mesoskopischen Skala auf epitaktischem Graphen

Eine endgültige theoretische Beschreibung und Erklärung zum lateralen Versatz des Potentialsprunges kann an dieser Stelle nicht gegeben werden. Der Verlauf des elektrochemischen Potentials an ML-BL-Übergängen kann aber für zukünftige theoretische Modellierungen genutzt werden, um ein adäquates Transportmodell über diese zu beschreiben und quantitativ mit den hier gewonnen Beobachtungen zu vergleichen. 


\section{Zusammenfassung und Ausblick}

In dieser Arbeit werden die lokalen elektronischen Eigenschaften sowie der Ladungstransport bis auf atomare Längenskalen von epitaktischem Graphen auf der SiC(0001)-Oberfläche charakterisiert. Dazu wird neben den etablierten Rastersondenverfahren erstmals bei $6 \mathrm{~K}$ und unter UHV-Bedingungen die Methode der Rastertunnelpotentiometrie eingesetzt.

Hierzu wurden epitaktisch gewachsene Graphenproben auf der 6H-Si(0001)-Oberfläche unter UHV-Bedingungen durch resistives Heizen präpariert und anschließend elektrisch kontaktiert. Mit Hilfe des Rasterkraftmikroskopie und niederenergetischen Elektronenbeugung wurde die Morphologie der Proben untersucht. Es konnten heterogene Proben mit einer Bedeckung von ein- und zweilagigem Graphen präpariert werden, die eine direkte vergleichende Untersuchung mit dem Rastertunnelmikroskop ermöglicht. Ergänzend konnte zur Bestimmung der Lagenanzahl der gebildeten Graphenschichten die Differenz des Oberflächenpotentials von ein- und zweilagigem Graphen an Atmosphäre durch die Raster-Kelvin-Mikroskopie ermittelt werden.

Für Transportexperimente und zukünftige Anwendungen spielt der Kontaktwiderstand zwischen epitaktisch gewachsenem Graphen und den kontaktierenden Elektroden eine entscheidende Rolle. Es ist erstmals gelungen, durch räumlich aufgelöste Messungen am Gold-Graphen-Interface auf semi-isolierenden SiC(0001) eine obere Grenze des Kontaktwiderstandes von $\rho_{\mathrm{c}}=1 \times 10^{-6} \Omega \mathrm{cm}^{2}$ abzuschätzen.

Die Untersuchung der epitaktisch gewachsenen Graphenproben mit der Methode der Rastertunnelmikroskopie ermöglichen die eindeutige Identifizierung von ein- und zweilagigem Graphen und deren hexagonale atomare Struktur, die über mehrere $100 \mathrm{~nm}^{2}$ keine Punktdefekte zeigen. Die unter der Graphenschicht liegende Zwischenschicht zeigt eine stark ungeordnete quasiperiodische Struktur mit zahlreichen Trimeren, die ebenso bei einer Bedeckung der Zwischenschicht mit ein- und zweilagigem Graphen abgebildet werden können. Einlagiges Graphen ist elektronisch auf atomaren Längenskalen im untersuchten Energiebereich stark inhomogen. Es konnten im Energiebereich von $\pm 100 \mathrm{mV}$ zahlreiche lokalisierte, räumlich variierende Zustände identifiziert werden, die selbst bei der Fermienergie auf Längenskalen von $5 \mathrm{~nm}^{2}$ zu Variationen in der Zustandsdichte führen. Auf 
zweilagigem Graphen sind Variationen in der lokalen Zustandsdichte geringer. Für die präparierten Proben konnte der Dirac-Punkt bei $320 \mathrm{meV}$ mit einer Bandlücke von $110 \mathrm{mV}$ identifiziert werden. Dies steht im Einklang mit bisherigen Untersuchungen auf diesem Probensystem. Um den für den elektronischen Transport relevanten Energiebereich bei $E_{\mathrm{F}}$ zu spektroskopieren, wurde der Effekt der Thermospannung im Tunnelkontakt ausgenutzt. Dies liefert neue Einblicke in die elektronische Struktur der Graphenoberfläche bei $E_{\mathrm{F}}$. Die räumliche Variation der Thermospannung bei abgeschätzten Temperaturdifferenzen von einigen 10 bis $100 \mathrm{~K}$ liegt bei einigen 10 bis $100 \mu \mathrm{V}$ sowohl auf atomarer Skala als auch zwischen ein- und zweilagigem Graphen und ist sehr empfindlich auf die atomaren Eigenschaften der eingesetzten STM-Spitze. Die hohe laterale und energetische Auflösung des Verfahrens ermöglicht die Analyse von Streuprozessen wie der Intra- und Intervalley-Streuung und zeigt im Gegensatz zu bisherigen Annahmen, dass auch noch zweilagiges Graphen elektronisch von der Zwischenschicht beeinflusst wird.

Die starke elektronische Inhomogenität der Proben bei der Fermi-Energie spiegelt sich auch in den Transportexperimenten mit der Rastertunnelpotentiometire wider. Es zeigen sich signifikante Spannungsabfälle auf ein- und zweilagigen Graphenflächen und an lokalisierten Defekten wie ML-ML und ML-BL-Übergängen. Der Potentialverlauf kann gut durch ein klassisches ohmsches Transportmodell mit spezifischen Widerständen beschrieben werden. Die quantitative Analyse liefert spezifische Widerstände der einzelnen Defekte, die in der Größenordnung bisheriger Transportuntersuchungen liegen. Dabei zeigt sich, dass einund zweilagiges epitaktisches Graphen nahezu identische Mobilitäten von $\sim 1000 \mathrm{~cm}^{2} / \mathrm{Vs}$ bzw. mittlere freie Weglängen von $\sim 40 \mathrm{~nm}$ bei $6 \mathrm{~K}$ aufweisen. Diese Werte liegen weit unter den theoretisch erwarteten einer defektfreien Graphenoberfläche.

Im Zuge der Transportmessungen wurde ebenso der Einfluss der Thermospannung im Tunnelkontakt untersucht. Für Ladungstransportmessungen stellt sie einen zunächst unerwünschten Nebeneffekt dar, da die Variationen in der Thermospannung in derselben GröBenordnung wie Variationen im lokalen elektrochemischen Potential im Fall eines Transportexperiments sind. Dies kann zu Fehlinterpretationen bei der Bestimmung von Spannungsabfällen führen. Jedoch konnte im Rahmen der experimentellen Auflösung gezeigt werden, dass sich die Thermospannung rein additiv verhält und für Messungen des lokalen elektrochemischen Potentials mit entgegensetzten Stromrichtungen eliminieren 
lässt. Dennoch ist die Auflösung der Ladungstransportmessungen durch die Thermospannung begrenzt.

Des Weiteren wurde der Verlauf von $\mu_{\mathrm{ec}, \mathrm{STP}}$ in der unmittelbaren Umgebung von Übergängen zwischen ein- und zweilagigem sowie einlagigem Graphen untersucht. Die Spannungsabfälle sind auf einen Bereich kleiner $\lambda_{\mathrm{F}} / 2$ lokalisiert. In Bezug auf den topographischen Verlauf zeigt sich für den Übergang zwischen ein- und zweilagigem Graphen ein lateraler Versatz hin zum zweilagigen Graphen. Als Ursache wird ein kombinierter Streumechanismus aus einer lokalen Änderung der Dotierung und Fehlanpassung der Wellenfunktionen am Übergang zwischen ein- und zweilagigem Graphen vorgeschlagen. Hier sind in Zukunft sowohl theoretische Berechnungen als auch Experimente nötig, die unter Berücksichtigung der Topologie zum einen die elektronische Struktur als auch die Transmission über solche Übergänge ermitteln.

Da gezeigt werden konnte, dass mit der hier verwendeten Rastertunnelpotetiometrie mikroskopische Einblicke in den Ladungstransport bei $6 \mathrm{~K}$ zu erhalten sind, stehen in Zukunft zahlreiche Möglichkeiten für weitere Studien offen. Um zum Beispiel den möglichen Einfluss der Zwischenschicht zu vermindern, ist in Zukunft die Untersuchung von Proben interessant, bei denen diese mit Wasserstoff passiviert worden ist. Wasserstoffpassivierte Proben zeigen außerdem eine wesentlich geringere Elektronenkonzentration, so dass möglicherweise auch lokal aufgelöste Transportmessungen nahe am Dirac-Punkt möglich werden. Eine weitere Möglichkeit den lokalen Ladungstransport in Abhängigkeit der Dotierkonzentration der Graphenschichten zu betrachten, ist es, die Lage der Fermienergie durch Dotierung der Graphenschichten mit zusätzlich aufgebrachten oder ins Graphengitter eingebauten Atomen wie z.B. Stickstoff gezielt zu verändern. Um einen Einblick in die räumliche Potentialverteilung in mesoskopische Transportregime zu erhalten und Quanteninterferenzeffekte wie z.B. die schwache Lokalisierung während eines Transportexperiments zu beobachten, sollen in Zukunft auf diesem Probensystem magnetfeldabhängige Ladungstransportmessungen mit der Rastertunnelpotentiometrie durchgeführt werden. 


\section{Anhang}

In diesem Anhang werden weitere Potentiometrie-Datensätze präsentiert, die mit Hilfe der in Kapitel 5.3.1 vorgestellten ohmschen Modellierung zur quantitativen Bestimmung der einzelnen Widerstandswerte, welche in Kapitel 5.3.1 in Abbildung 58 herangezogen wurden. In den Abbildungen 65 bis 70 sind jeweils in (a) die Topographie, in (b) das über die Rastertunnelpotentiometrie bestimmte, thermospannungsbereinigte lokale elektrochemische Potential, in (c) die nach Kapitel 5.3.1 beschriebene über die ohmsche Modellierung erhaltene Potentialverteilung und in (d) die Differenz der experimentell bestimmten und berechneten Potentialverteilung dargestellt. Die zugehörigen ermittelten Widerstände der einzelnen Defekte sind in den jeweiligen Bildunterschriften zu finden.

a)

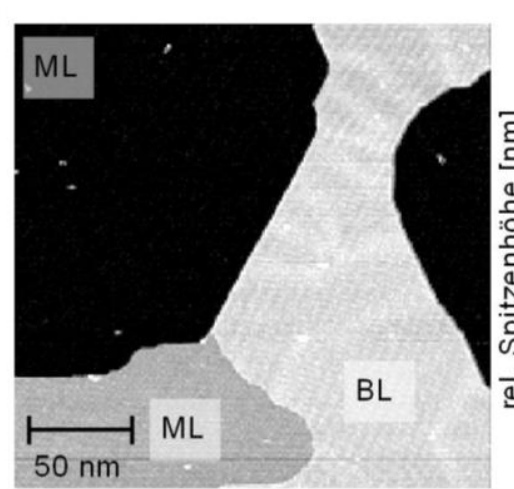

c)

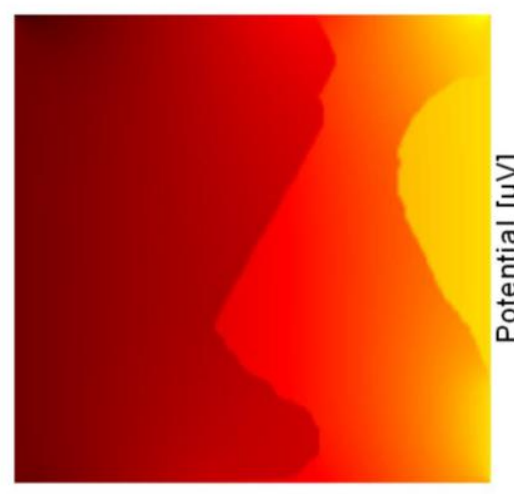

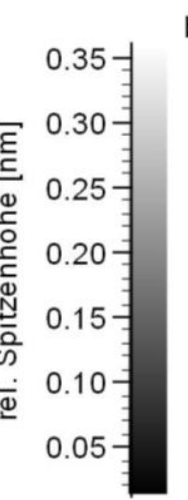

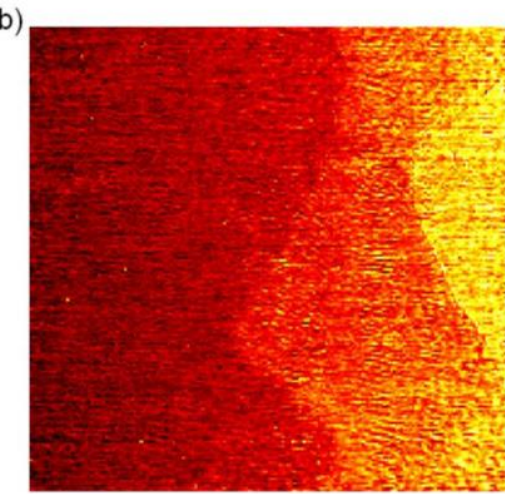

d)

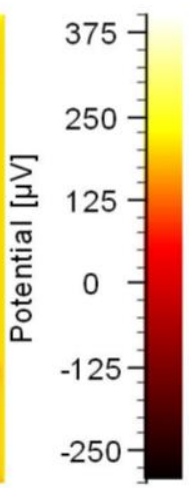

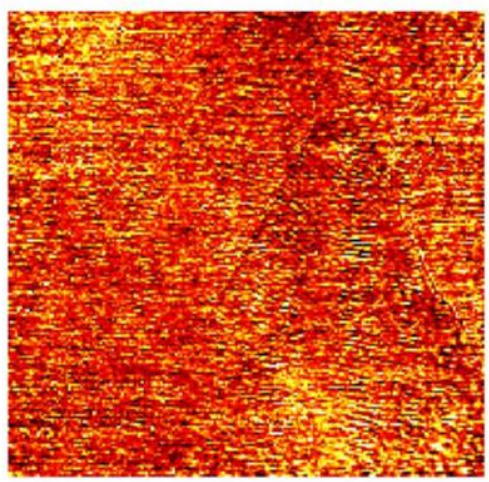
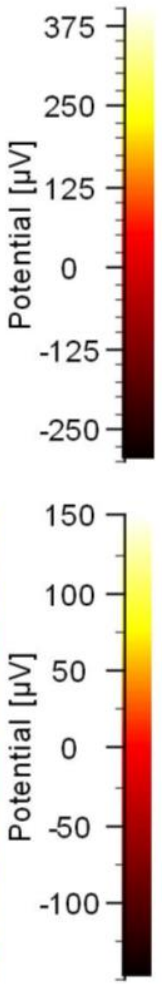

Abbildung 65: (a) Topographie, $\left(236 \times 252 \mathrm{~nm}^{2}, 200 \times 200\right.$ Pixel, $\left.U_{\text {Bias }}=30 \mathrm{mV}, I_{\mathrm{T}}=175 \mathrm{pA}\right)$, (b) experimentelle Potentialverteilung, (c) Simulationsergebnis mit $\rho_{\mathrm{ML}-\mathrm{BL} \text { I }}=55,6 \Omega \mu \mathrm{m}, \rho_{\mathrm{ML}-\mathrm{BL} \mathrm{II}}=117 \Omega \mu \mathrm{m}, \rho_{\mathrm{ML}}=$ $352 \Omega / \square$ und $\rho_{\mathrm{BL}}=637 \Omega / \square$ und (d) Differenz von (b) und (c). 
a)

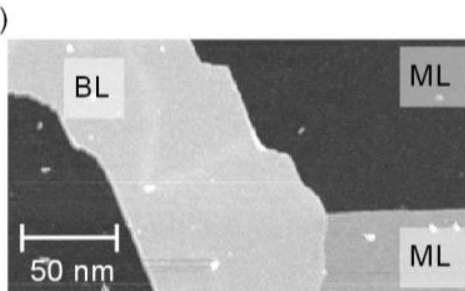

c)

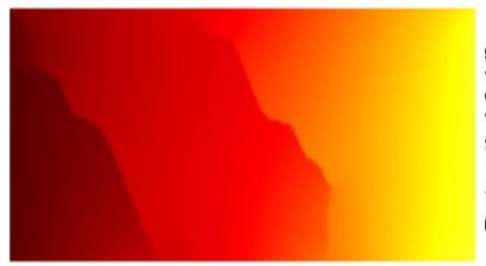

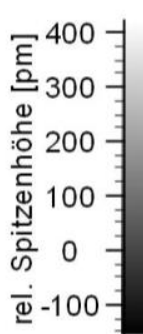

b)

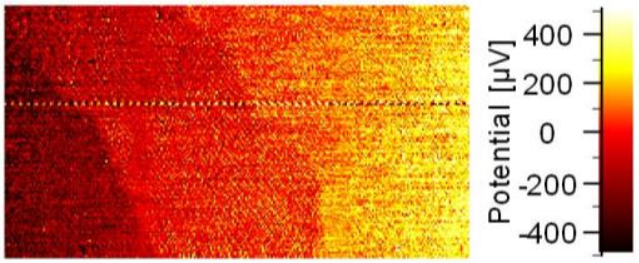

d)
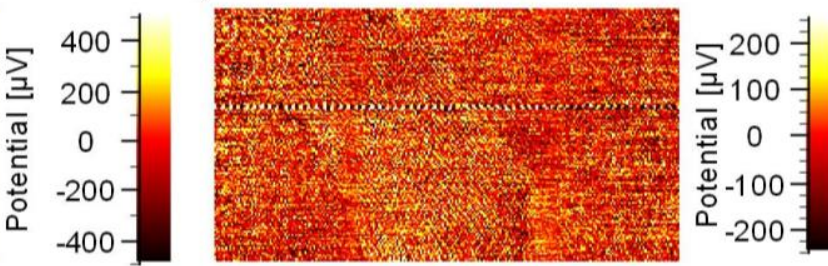

Abbildung 66: (a) Topographie, $\left(250 \times 119 \mathrm{~nm}^{2}, 253 \times 139\right.$ Pixel, $\left.U_{\text {Bias }}=-20 \mathrm{mV}, I_{\mathrm{T}}=350 \mathrm{pA}\right),(\mathrm{b})$ experimentelle Potentialverteilung, (c) Simulationsergebnis mit $\rho_{\mathrm{ML}-\mathrm{BLI}}=91 \Omega \mu \mathrm{m}, \rho_{\mathrm{ML}-\mathrm{BL} \mathrm{II}}=9 \Omega \mu \mathrm{m}, \rho_{\mathrm{ML}}=$ $559 \Omega / \square$ und $\rho_{\mathrm{BL}}=682 \Omega / \square$ und (d) Differenz von (b) und (c).
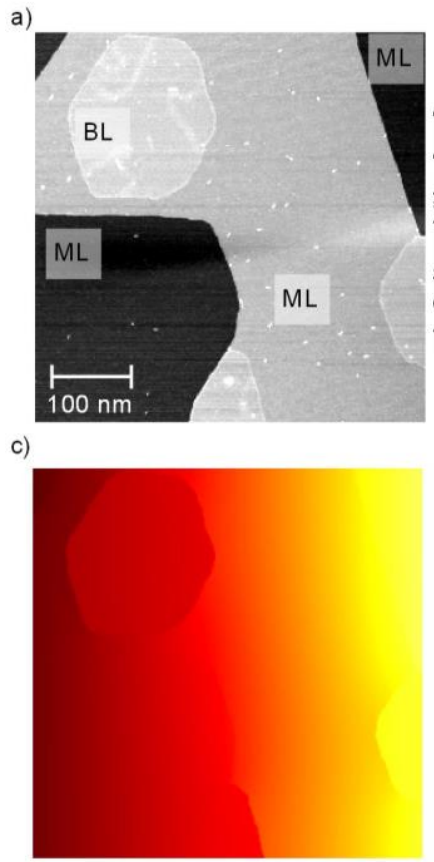
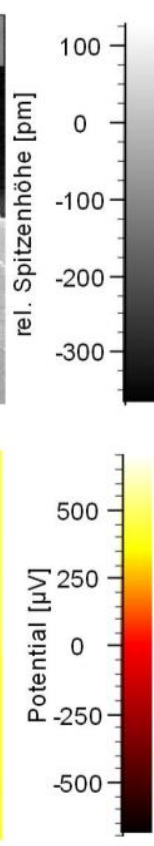
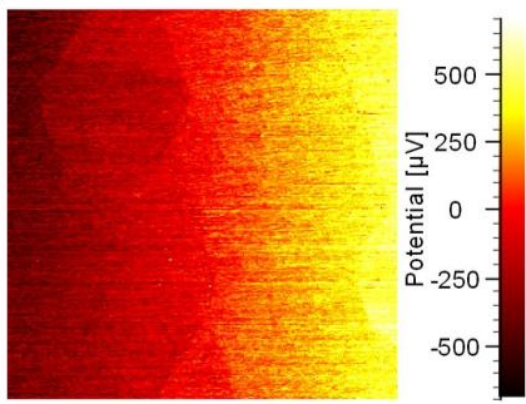

d)

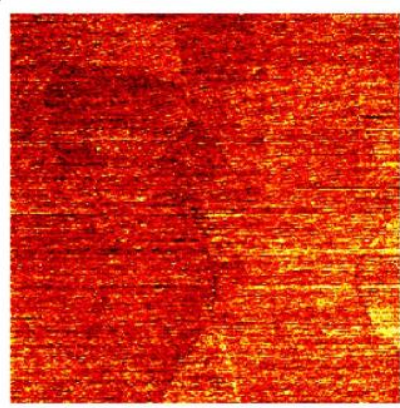

Abbildung 67: (a) Topographie $\left(500 \times 500 \mathrm{~nm}^{2}, 256 \times 256\right.$ Pixel, $\left.\mathrm{I}_{\mathrm{T}}=0,15 \mathrm{nA}, \mathrm{U}_{\mathrm{B}}=-0,03 \mathrm{~V}\right)$, (b) experimentelle Potentialverteilung, (c) Simulationsergebnis mit $\rho_{\mathrm{ML}-\mathrm{BLI}}=39 \Omega \mu \mathrm{m}, \rho_{\mathrm{ML}-\mathrm{BL} \mathrm{II}}=12 \Omega \mu \mathrm{m}, \rho_{\mathrm{ML}-\mathrm{MLI}}=$ $8 \Omega \mu \mathrm{m}, \rho_{\mathrm{ML}}=343 \Omega / \square$ und $\rho_{\mathrm{BL}}=189 \Omega / \square$ und (d) Differenz von (b) und (c). 
a)

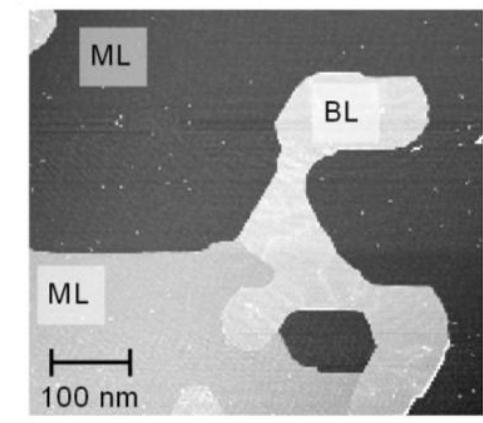

c)

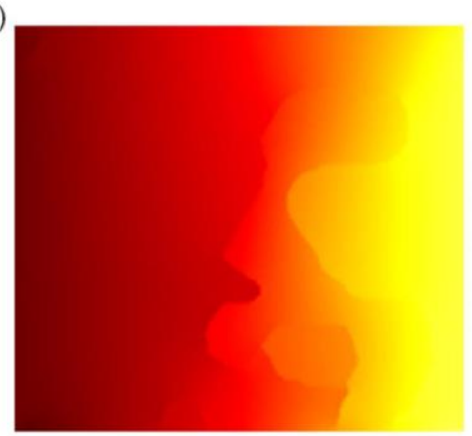

b)
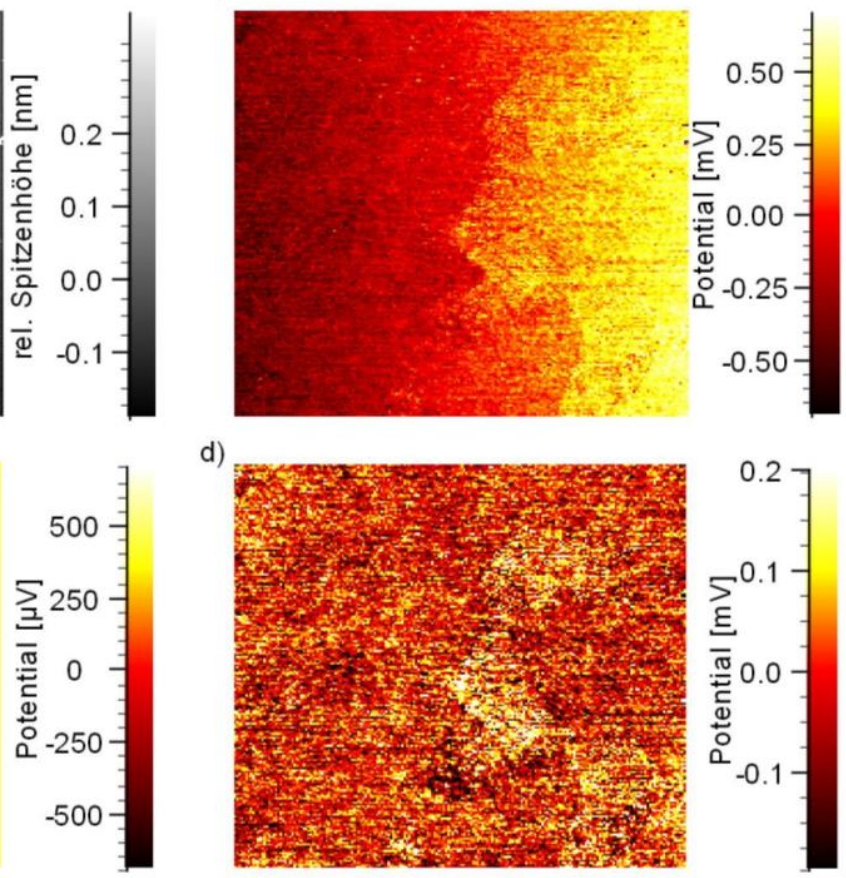

d)

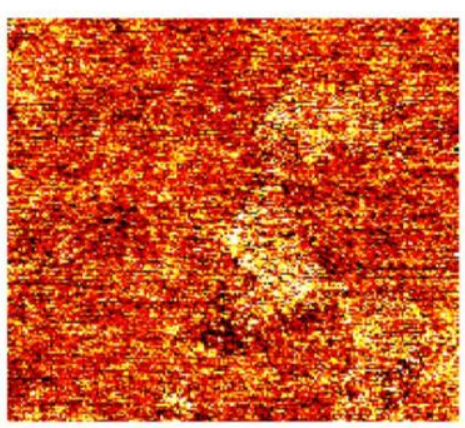

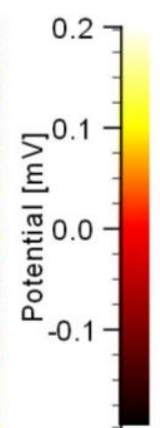

Abbildung 68: Topographie $\left(624 \times 548 \mathrm{~nm}^{2}, 200 \times 200\right.$ Pixel, $\left.U_{\mathrm{Bias}}=30 \mathrm{mV}, I_{\mathrm{T}}=175 \mathrm{pA}\right)$, (b) experimentelle Potentialverteilung, (c) Simulationsergebnis mit $\rho_{\mathrm{ML}-\mathrm{BL} \mathrm{I}}=37 \Omega \mu \mathrm{m}, \quad \rho_{\mathrm{ML}-\mathrm{BL} \mathrm{II}}=252 \Omega \mu \mathrm{m}, \quad \rho_{\mathrm{ML}}=$ $398 \Omega / \square$ und $\rho_{\mathrm{BL}}=946 \Omega / \square$ und (d) Differenz von (b) und (c).

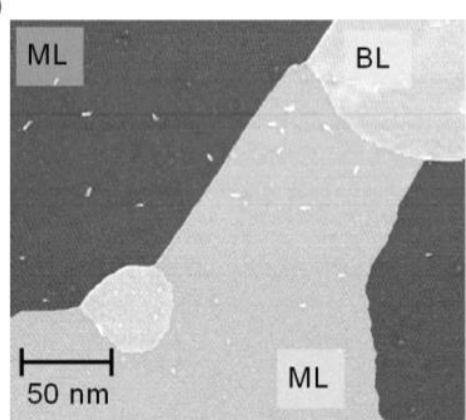

c)

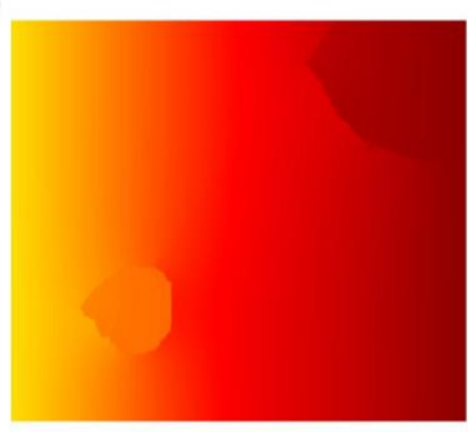

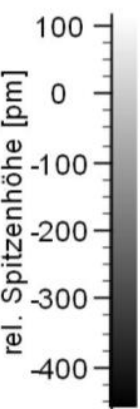

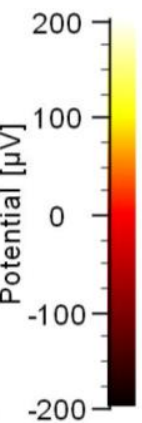

b)
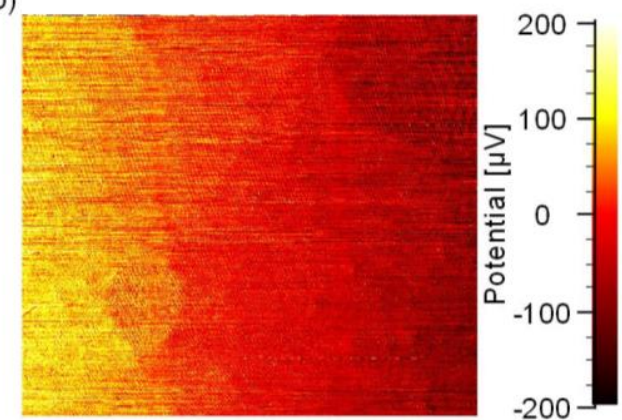

d)

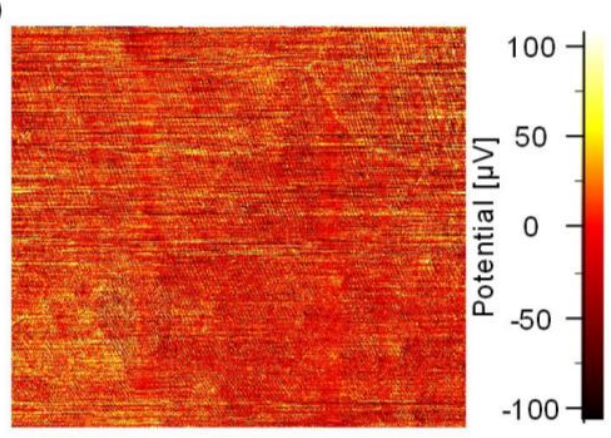

Abbildung 69: Topographie $\left(257 \times 228 \mathrm{~nm}^{2}, 507 \times 511\right.$ Pixel, $\left.U_{\mathrm{Bias}}=30 \mathrm{mV}, I_{\mathrm{T}}=175 \mathrm{pA}\right)$, (b) experimentelle Potentialverteilung, (c) Simulationsergebnis mit $\rho_{\mathrm{ML}-\mathrm{BL} \mathrm{I}}=11 \Omega \mu \mathrm{m}, \rho_{\mathrm{ML}-\mathrm{BL} \mathrm{II}}=69 \Omega \mu \mathrm{m}, \rho_{\mathrm{ML}-\mathrm{ML} \mathrm{I}}=$ $1,5 \Omega \mu \mathrm{m}, \rho_{\mathrm{ML}}=484 \Omega / \square$ und $\rho_{\mathrm{BL}}=333 \Omega / \square$ und (d) Differenz von (b) und (c). 
a)

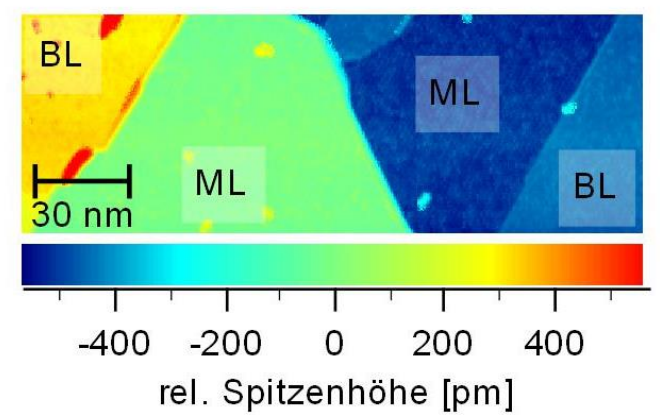

c)

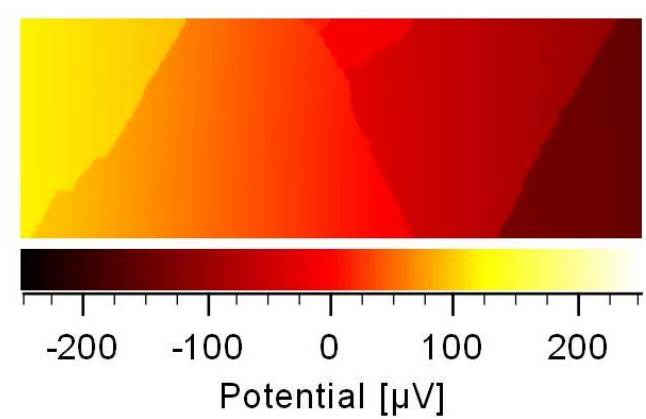

b)

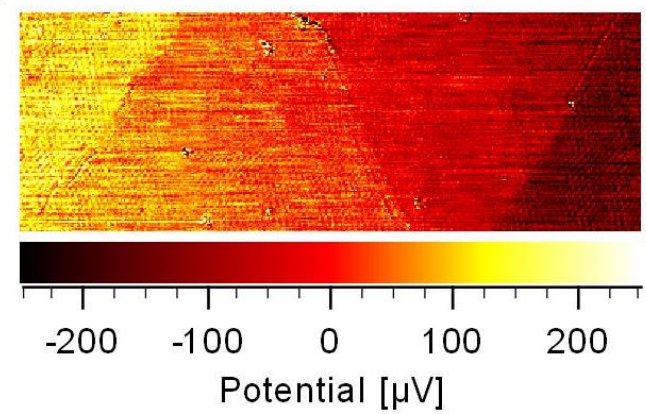

d)

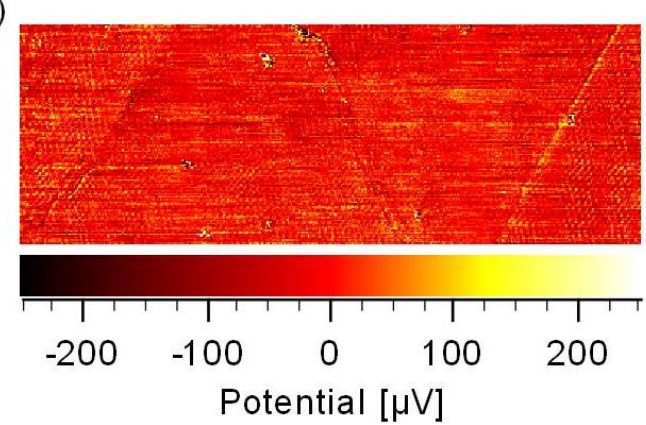

Abbildung 70: Topographie $\left(197 \times 37 \mathrm{~nm}^{2}, 395 \times 146\right.$ Pixel, $\left.U_{\mathrm{Bias}}=-70 \mathrm{mV}, I_{\mathrm{T}}=580 \mathrm{pA}\right)$, (b) experimentelle Potentialverteilung, (c) Simulationsergebnis mit $\rho_{\mathrm{ML}-\mathrm{BL} \mathrm{I}}=26 \Omega \mu \mathrm{m}, \rho_{\mathrm{ML}-\mathrm{BL} \mathrm{II}}=49 \Omega \mu \mathrm{m}, \rho_{M \mathrm{~L}-\mathrm{ML} \mathrm{II}}=$ 33,9 $\Omega \mu \mathrm{m}, \rho_{\mathrm{ML}}=681 \Omega / \square$ und $\rho_{\mathrm{BL}}=314 \Omega / \square$ und (d) Differenz von (b) und (c). 


\section{Literaturverzeichnis}

[1] http://www.itrs.net, 2012.

[2] T. Shinada, S. Okamoto, T. Kobayashi, and I. Ohdomari. Enhancing semiconductor device performance using ordered dopant arrays. Nature, 437(7062):1128-1131, 2005.

[3] K. Teichmann, M. Wenderoth, S. Loth, R. G. Ulbrich, J. K. Garleff, A. P. Wijnheijmer, and P. M. Koenraad. Controlled charge switching on a single donor with a scanning tunneling microscope. Phys. Rev. Lett., 101(7):076103-, 2008.

[4] C. Tao, W. G. Cullen, and E. D. Williams. Visualizing the electron scattering force in nanostructures. Science, 328(5979):736-740, 2010.

[5] P. Muralt and D. W. Pohl. Scanning tunneling potentiometry. Applied Physics Letters, 48(8):514-516, 1986.

[6] A. Bannani, C. A. Bobisch, and R. Möller. Local potentiometry using a multiprobe scanning tunneling microscope. Review of Scientific Instruments, 79(8):-, 2008.

[7] J. R. Kirtley, S. Washburn, and M. J. Brady. Direct measurement of potential steps at grain boundaries in the presence of current flow. Phys. Rev. Lett., 60(15):1546-1549, 1988.

[8] J. P. Pelz and R. H. Koch. Extremely low noise potentiometry with a scanning tunneling microscope. Review of Scientific Instruments, 60(3):301-305, 1989.

[9] M. Rozler and M. R. Beasley. Design and performance of a practical variable-temperature scanning tunneling potentiometry system. Review of Scientific Instruments, 79(7):-, 2008.

[10] P. R. Wallace. The band theory of graphite. Phys. Rev., 71(9):622-634, 1947.

[11] R. Saito, G. Dresselhaus, and M.S. Dresselhaus. Physical Properties of Carbon Nanotubes. IMPERIAL COLLEGE PRESS AND DISTRIBUTED BY WORLD SCIENTIFIC PUBLISHING CO., 1998.

[12] C. Bena and G. Montambaux. Remarks on the tight-binding model of graphene. New Journal of Physics, 11(9):095003-, 2009.

[13] E. McCann. Graphene Nanoelectronics - Metrology, Synthesis, Properties and Aplication.

[14] T. Ando, T. Nakanishi, and R. Saito. Berry's phase and absence of back scattering in carbon nanotubes. J. Phys. Soc. Jpn., 67:2857, 1998.

[15] P. L. McEuen, M. Bockrath, D. H. Cobden, Y.-G. Yoon, and S. G. Louie. Disorder, pseudospins, and backscattering in carbon nanotubes. Phys. Rev. Lett., 83(24):5098-5101, 1999. 
[16] A. Luican, Guohong Li, A. Reina, J. Kong, R. R. Nair, K. S. Novoselov, A. K. Geim, and E. Y. Andrei. Single-layer behavior and its breakdown in twisted graphene layers. Phys. Rev. Lett., 106(12):126802-, 2011.

[17] E. McCann and V. I. Falko. Landau-level degeneracy and quantum hall effect in a graphite bilayer. Phys. Rev. Lett., 96(8):086805-, 2006.

[18] E. McCann, D. S. L. Abergel, and V. I. Falko. Electrons in bilayer graphene. Solid State Communications, 143(1-2):110-115, 2007.

[19] T. Ohta, A. Bostwick, J. L. McChesney, T. Seyller, K. Horn, and E. Rotenberg. Interlayer interaction and electronic screening in multilayer graphene investigated with angle-resolved photoemission spectroscopy. Phys. Rev. Lett., 98(20):206802-, 2007.

[20] J. Bardeen. Tunneling from many-particle point of view. Phys. Rev. Lett., 6:57-59, 1991

[21] J. Tersoff and D. R. Hamann. Theory of the scanning tunnelling microscope. Phys. Rev. B, 31:805-813, 1985.

[22] R. J. Hamers. Atomic-resolution surface spectroscopy with the scanning tunnelling microscope. Annual Review of Physical Chemnistry, 40:531-559, 1989

[23] N. D. Lang. Spectroscopy of single atoms in the scanning tunnelling microscope. Phsy. Rev. B, 34: 5947-5950, 1986

[24] T. Ihn. Semiconductor nanostructures: quantum states and electronic transport. Oxford Univ. Press, 2010

[25] A. Mahmood, C. Naud, C. Bouvier, F. Hiebel, P. Mallet, J.-Y. Veuillen, L. P. Levy, D. Chaussende, and T. Ouisse. Epitaxial graphene morphologies probed by weak (anti)-localization. Journal of Applied Physics, 113(8):-, 2013.

[26] C. W. J. Beenakker and H. van Houten. Quantum transport in semiconductor nanostructures. In Henry Ehrenreich and David Turnbull, editors, Semiconductor Heterostructures and Nanostructures, volume Volume 44, pages 1-228. Academic Press, 1991.

[27] M. Di Ventra. Electrical Transport in Nanoscale Systems. Cambridge University Press, 2008.

[28] M. J. McLennan, Y. Lee, and S. Datta. Voltage drop in mesoscopic systems: A numerical study using a quantum kinetic equation. Phys. Rev. B, 43(17):13846-13884, 1991.

[29] S. Datta. Electronic Transport in Mesoscopic Systems (Cambridge Studies in Semiconductor Physics and Microelectronic Engineering). Cambridge University Press, May 1997.

[30] A. Marx R. Gross. Festkörperphysik. Oldenbourg Verlag, 2012.

[31] Y. Imry and R. Landauer. Conductance viewed as transmission. Rev. Mod. Phys., 71(2):S306S312, 1999. 
[32] K. Besocke. An easily operable scanning tunneling microscope. Surface Science, 181(12):145-153, March 1987.

[33] K. Teichmann. Scanning tunneling spectroscopy of space charge regions in semiconductors: From single donor to heterostructure systems. PhD thesis, Universität Göttingen, 2012.

[34] T. Druga, M. Wenderoth, J. Homoth, M. A. Schneider, and R. G. Ulbrich. A versatile high resolution scanning tunneling potentiometry implementation. Review of Scientific Instruments, 81(8):-, 2010.

[35] H. Birk, M. J. M. de Jong, and C. Schönenberger. Shot-noise suppression in the single electron tunneling regime. Phys. Rev. Lett., 75(8):1610-1613, 1995.

[36] H. Nyquist. Thermal agitation of electric charge in conductors. Phys. Rev., 32(1):110-113, July 1928.

[37] J. B. Johnson. Thermal agitation of electricity in conductors. Phys. Rev., 32(1):97-109, 1928.

[38] X. Z. Yu, C.G. Hwang, C.M. Jozwiak, A. Köhl, A.K. Schmid, and A. Lanzara. New synthesis method for the growth of epitaxial graphene. Journal of Electron Spectroscopy and Related Phenomena, 184(3-6):100-106, 2011.

[39] J. Hass, F. Varchon, J. E. Millan-Otoya, M. Sprinkle, N. Sharma, W. A. de Heer, C. Berger, P. N. First, L. Magaud, and E. H. Conrad. Why multilayer graphene on $4 \mathrm{H}-$ $\mathrm{SiC}(0001)$ behaves like a single sheet of graphene. Phys. Rev. Lett., 100(12):125504-, 2008 .

[40] A.J. Van Bommel, J.E. Crombeen, and A. Van Tooren. Leed and auger electron observations of the SiC(0001) surface. Surface Science, 48(2):463-472, 1975.

[41] K. V. Emtsev, F. Speck, Th. Seyller, L. Ley, and J. D. Riley. Interaction, growth, and ordering of epitaxial graphene on $\mathrm{SiC}(0001)$ surfaces: A comparative photoelectron spectroscopy study. Phys. Rev. B, 77(15):155303-, 2008.

[42] I. Forbeaux, J.-M. Themlin, and J.-M. Debever. Heteroepitaxial graphite on 6H-SiC(0001): Interface formation through conduction-band electronic structure. Phys. Rev. B, 58(24):16396-16406, 1998.

[43] Fredrik Owman and Per Martensson. Stm study of the SiC(0001) surface. Surface Science, 330(1):L639-L645, 1995.

[44] A. N. Hattori, T. Okamoto, S. Sadakuni, J. Murata, K. Arima, Y.Y. Sano, K. Hattori, H. Daimon, K. Endo, and K. Yamauchi. Formation of wide and atomically flat graphene layers on ultraprecision-figured 4H-SiC(0001) surfaces. Surf. Sci., 605(5-6):597-605, 2011.

[45] H. Hibino, H. Kageshima, and M. Nagase. Epitaxial few-layer graphene: towards single crystal growth. Journal of Physics D: Applied Physics, 43(37):374005-, 2010. 
[46] H. Huang, W. Chen, S. Chen, and A. T. S. Wee. Bottom-up growth of epitaxial graphene on 6H-SiC(0001). ACS Nano, 2(12):2513-2518, 2008.

[47] P. Lauffer, K. V. Emtsev, R. Graupner, Th. Seyller, L. Ley, S. A. Reshanov, and H. B. Weber. Atomic and electronic structure of few-layer graphene on $\mathrm{SiC}(0001)$ studied with scanning tunneling microscopy and spectroscopy. Phys. Rev. B, 77(15):155426, 2008.

[48] M. Hupalo, E. H. Conrad, and M. C. Tringides. Growth mechanism for epitaxial graphene on vicinal $6 \mathrm{H}-\mathrm{SiC}(0001)$ surfaces: A scanning tunneling microscopy study. Phys. Rev. B, 80(4):041401-, 2009.

[49] H. Hibino, S. Tanabe, S. Mizuno, and H. Kageshima. Growth and electronic transport properties of epitaxial graphene on SiC. Journal of Physics D: Applied Physics, 45(15):154008-, 2012.

[50] K. V. Emtsev, A. Bostwick, K. Horn, J. Jobst, G. L. Kellogg, L. Ley, J. L. McChesney, T. Ohta, S. A. Reshanov, J. Rohrl, E. Rotenberg, A. K. Schmid, D. Waldmann, H. B.Weber, and T. Seyller. Towards wafer-size graphene layers by atmosphericpressure graphitization of silicon carbide. Nat Mater, 8(3):203-207, 2009.

[51] W. A. de Heer, C. Berger, M. Ruan, M. Sprinkle, X. Li, Y. Hu, B. Zhang, J. Hankinson, and E. Conrad. Large area and structured epitaxial graphene produced by confinement controled sublimation of silicon carbide. Proceedings of the National Academy of Sciences, 108(41):16900-16905, 2011.

[52] M. H. Oliveira, Jr., T. Schumann, M. Ramsteiner, J. M. J. Lopes, and H. Riechert. Influence of the silicon carbide surface morphology on the epitaxial graphene formation. Appl. Phys. Lett., 99(11):111901-3, 2011.

[53] T. Filleter, K. V. Emtsev, Th. Seyller, and R. Bennewitz. Local work function measurements of epitaxial graphene. Appl. Phys. Lett., 93(13):133117-3, 2008.

[54] J. Hass, J. E. Millan-Otoya, P. N. First, and E. H. Conrad. Interface structure of epitaxial graphene grown on 4H-SiC(0001). Phys. Rev. B, 78(20):205424-, 2008.

[55] Y. Baskin and L. Meyer. Lattice constants of graphite at low temperatures. Phys. Rev., 100(2):544-544, 1955.

[56] F. Varchon, R. Feng, J. Hass, X. Li, B. Ngoc Nguyen, C. Naud, P. Mallet, J.-Y. Veuillen, C. Berger, E. H. Conrad, and L. Magaud. Electronic structure of epitaxial graphene layers on SiC: Effect of the substrate. Phys. Rev. Lett., 99(12):126805-, 2007.

[57] W. Chen, H. Xu, L. Liu, X. Gao, D. Qi, G. Peng, S. C. Tan, Y. Feng, K. P. Loh, and A. T. S. Wee. Atomic structure of the 6H-SiC(0001) nanomesh. Surface Science, 596(1-3):176186, 2005. 
[58] W. J. Ong and E. S. Tok. Role of si clusters in the phase transformation and formation of (6 $\tilde{\mathrm{A}}-6)$-ring structures on $6 \mathrm{H}-\mathrm{SiC}(0001)$ as a function of temperature: An stm and xps study. Phys. Rev. B, 73(4):045330-, 2006.

[59] Y. Qi, S. H. Rhim, G. F. Sun, M. Weinert, and L. Li. Epitaxial graphene on SiC(0001): More than just honeycombs. Phys. Rev. Lett., 105(8):085502-, 2010.

[60] G. M. Rutter, N. P. Guisinger, J. N. Crain, E. A. A. Jarvis, M. D. Stiles, T. Li, P. N. First, and J. A. Stroscio. Imaging the interface of epitaxial graphene with silicon carbide via scanning tunneling microscopy. Phys. Rev. B, 76(23):235416-, 2007.

[61] F. Varchon, P. Mallet, J.-Y. Veuillen, and L. Magaud. Ripples in epitaxial graphene on the Siterminated $\mathrm{SiC}(0001)$ surface. Phys. Rev. B, 77(23):235412-, 2008.

[62] C. Riedl, U. Starke, J. Bernhardt, M. Franke, and K. Heinz. Structural properties of the graphene-SiC $(0001)$ interface as a key for the preparation of homogeneous large terrace graphene surfaces. Phys. Rev. B, 76(24):245406-, 2007.

[63] Gabriele Sclauzero and Alfredo Pasquarello. Stability and charge transfer at the interface between $\mathrm{SiC}(0001)$ and epitaxial graphene. Microelectronic Engineering, 88(7):1478-1481, 2011.

[64] S. W. Poon. Dynamics of Epitaxial Graphene growth and adsorptions of Cobalt. PhD thesis, Physics Depatement National University of Singapore, 2010.

[65] T. W. Hu, F. Ma, D. Y. Ma, D. Yang, X. T. Liu, K. W. Xu, and Paul K. Chu. Evidence of atomically resolved $6 \times 6$ buffer layer with long-range order and short-range disorderduring formation of graphene on $6 \mathrm{H}-\mathrm{SiC}$ by thermal decomposition. Appl. Phys. Lett., 102(17):171910-4, 2013.

[66] T. Ohta, A. Bostwick, Th. Seyller, K. Horn, and E. Rotenberg. Controlling the electronic structure of bilayer graphene. Science, 313(5789):951-954, 2006.

[67] J. Ristein, S. Mammadov, and Th. Seyller. Origin of doping in quasi-free-standing graphene on silicon carbide. Phys. Rev. Lett., 108(24):246104-, 2012.

[68] A. Bostwick, T. Ohta, T. Seyller, K. Horn, and E. Rotenberg. Quasiparticle dynamics in graphene. Nat Phys, 3(1):36-40, 2007.

[69] S. Kopylov, A. Tzalenchuk, S. Kubatkin, and V. I. Falko. Charge transfer between epitaxial graphene and silicon carbide. Appl. Phys. Lett., 97(11):112109-3, 2010.

[70] F. Owman and P. Martensson. The SiC(0001) reconstruction studied with STM and LEED. Surface Science, 369(1-3):126-136, 1996.

[71] C. Riedl, A. A. Zakharov, and U. Starke. Precise in situ thickness analysis of epitaxial graphene layers on $\mathrm{SiC}(0001)$ using low-energy electron diffraction and angle resolved ultraviolet photoelectron spectroscopy. Applied Physics Letters, 93(3):033106, 2008. 
[72] A. Ferreira da Silva, J. Pernot, S. Contreras, B. E. Sernelius, C. Persson, and J. Camassel. Electrical resistivity and metal-nonmetal transition in n-type doped 4H-SiC. Phys. Rev. B, 74(24):245201-, 2006.

[73] H. Hibino, H. Kageshima, M. Kotsugi, F. Maeda, F.-Z. Guo, and Y. Watanabe. Dependence of electronic properties of epitaxial few-layer graphene on the number of layers investigated by photoelectron emission microscopy. Phys. Rev. B, 79(12):125437-, 2009.

[74] T. L. Burnett, R. Yakimova, and O. Kazakova. Identification of epitaxial graphene domains and adsorbed species in ambient conditions using quantified topography measurements. $J$. Appl. Phys., 112(5):054308-7, 2012.

[75] R. Pearce, J. Eriksson, T. Iakimov, L. Hultman, A. Lloyd Spetz, and R. Yakimova. On the differing sensitivity to chemical gating of single and double layer epitaxial graphene explored using scanning kelvin probe microscopy. ACS Nano, 7(5):4647-4656, 2013.

[76] O. Kazakova, V. Panchal, and T. Burnett. Epitaxial graphene and graphen based devices studied by electrical scanning probe microscopy. Crystals, 3(1):191-233, 2013.

[77] P. Martensson, F. Owman, and L. I. Johansson. Morphology, atomic and electronic structure of 6H-SiC(0001) surfaces. phys. stat. sol. (b), 202(1):501-528, 1997.

[78] S. Nie and R. M. Feenstra. Tunneling spectroscopy of graphene and related reconstructions on SiC(0001). In J. Vac. Sci. Technol. A, volume 27, pages 1052-1057. AVS, 2009.

[79] J. Cervenka, K. van de Ruit, and C. F. J. Flipse. Giant inelastic tunneling in epitaxial graphene mediated by localized states. Phys. Rev. B, 81(20):205403-, 2010.

[80] J. Cervenka, K. van der Ruit, and Kees Flipse. Effect of local doping on the electronic properties of epitaxial graphene on SiC. phys. stat. sol. (a), 207(3):595-598, 2010.

[81] V. W. Brar, Y. Zhang, Y. Yayon, T. Ohta, J. L. McChesney, A. Bostwick, E. Rotenberg, K. Horn, and M. F. Crommie. Scanning tunneling spectroscopy of inhomogeneous electronic structure in monolayer and bilayer graphene on SiC. Appl. Phys. Lett., 91(12):122102-3, 2007.

[82] L. Vitali, C. Riedl, R. Ohmann, I. Brihuega, U. Starke, and K. Kern. Spatial modulation of the dirac gap in epitaxial graphene. Surface Science, 602(22):L127-L130, 2008.

[83] Y. Zhang, V. W. Brar, F. Wang, C. Girit, Y. Yayon, M. Panlasigui, A. Zettl, and M. F. Crommie. Giant phonon-induced conductance in scanning tunnelling spectroscopy of gate-tunable graphene. Nat. Phys., 4(8):627-630, 2008.

[84] P. Mallet, F. Varchon, C. Naud, L. Magaud, C. Berger, and J.-Y. Veuillen. Electron states of mono- and bilayer graphene on SiC probed by scanning-tunneling microscopy. Phys. Rev. B, 76(4):041403-, 2007. 
[85] K. S. Kim, T.-H. Kim, A. L. Walter, T. Seyller, H. W. Yeom, E. Rotenberg, and A. Bostwick. Visualizing atomic-scale negative differential resistance in bilayer graphene. Phys. Rev. Lett., 110(3):036804-, 2013.

[86] P. Mallet, I. Brihuega, S. Bose, M. M. Ugeda, J. M. Gómez-Rodriguez, K. Kern, and J. Y. Veuillen. Role of pseudospin in quasiparticle interferences in epitaxial graphene probed by high-resolution scanning tunneling microscopy. Phys. Rev. B, 86(4):045444-, 2012.

[87] S. Goler, C. Coletti, V. Piazza, P. Pingue, F. Colangelo, V. Pellegrini, K. V. Emtsev, S. Forti, U. Starke, F. Beltram, and S. Heun. Revealing the atomic structure of the buffer layer between $\mathrm{SiC}(0001)$ and epitaxial graphene. Carbon, 51(0):249-254, 2013.

[88] N. Ferralis, R. Maboudian, and C. Carraro. Evidence of structural strain in epitaxial graphene layers on 6H-SiC(0001). Phys. Rev. Lett., 101(15):156801, 2008.

[89] S. Cho, S. D. Kang, W. Kim, Eui-Sup Lee, Sung-Jae Woo, Ki.-J. Kong, I. Kim, H.- D. Kim, T. Zhang, J. A. Stroscio, Y.-H. Kim, and H.-K. Lyeo. Thermoelectric imaging of structural disorder in epitaxial graphene. Nat. Mater., 12(10):913-918, 2013.

[90] L. Bürgi, O. Jeandupeux, H. Brune, and K. Kern. Probing hot-electron dynamics at surfaces with a cold scanning tunneling microscope. Phys. Rev. Lett., 82(22):4516-4519, 1999.

[91] M. F. Crommie, C. P. Lutz, and D. M. Eigler. Imaging standing waves in a two-dimensional electron gas. Nature, 363(6429):524-527, 1993.

[92] Oersted. Notiz von neuen electrisch - magnetischen Versuchen des Herrn Seebeck in Berlin. Ann. Phys., 73(4):430-432, 1823.

[93] von Yelin. Der Thermomagnetismus der Metalle, eine neue Entdeckung. Ann. Phys., 73(4):415-429, 1823.

[94] O. I. Shklyarevskii, A. G. M. Jansen, J. G. H. Hermsen, and P. Wyder. Thermoelectric voltage between identical metals in a point-contact configuration. Phys. Rev. Lett., 57(11):13741377, 1986.

[95] M. Kohler. Thermischer Kontaktwiderstand von Metallen und der dadurch verursachte temperatursprung im Kontakt. Neuartige Thermokräfte im einmetallischen Kreis und ihr Zusammenhang mit dem Benedickseffekt. Ann. Phys., 430(7-8):542-554, 1940.

[96] C.R. Leavens and G.C. Aers. Vacuum tunnelling thermopower: Normal metal electrodes. Solid State Communications, 61(5):289-295, 1987.

[97] J. A. Stovneng and P. Lipavsky. Thermopower in scanning-tunneling-microscope experiments. Phys. Rev. B, 42(14):9214-9216, 1990.

[98] C. C. Williams and H. K. Wickramasinghe. Scanning thermal profiler. Appl. Phys. Lett., 49(23):1587-1589, 1986. 
[99] C. C. Williams and H. K. Wickramasinghe. Microscopy of chemical-potential variations on an atomic scale. Nature, 344(6264):317-319, 1990.

[100] C. C. Williams and H. K. Wickramasinghe. Scanning chemical potential microscope: A new technique for atomic scale surface investigation. In J. Vac. Sci. Technol. B, volume 9, pages 537-540, Boston, Massachusetts (USA), 1991. AVS.

[101] D. Hoffmann, A. Haas, T. Kunstmann, J. Seifritz, and R. Möller. Thermovoltage in scanning tunneling microscopy. In J. Vac. Sci. Technol. A, volume 15, pages 1418-1422, Philadelphia, Pennsylvania (USA), 1997. AVS.

[102] D. Hoffmann, J. Seifritz, B. Weyers, and R. Möller. Thermovoltage in scanning tunneling microscopy. Journal of Electron Spectroscopy and Related Phenomena, 109(1-2):117-125, 2000.

[103] A. Rettenberger, C. Baur, K. Lauger, D. Hoffmann, J. Y. Grand, and R. Möller. Variation of the thermovoltage across a vacuum tunneling barrier: Copper islands on $\mathrm{Ag}$ (111). Appl. Phys. Lett., 67(9):1217-1219, 1995.

[104] K. J. Engel, M. Wenderoth, N. Quaas, T. C. G. Reusch, K. Sauthoff, and R. G. Ulbrich. Thermovoltage mapping of standing electron waves on $\mathrm{Au}(111)$ surfaces at low temperatures. Phys. Rev. B, 63(16):165402-, 2001.

[105] D. Hoffmann, J. Y. Grand, R. Möller, A. Rettenberger, and K. Läuger. Thermovoltage across a vacuum barrier investigated by scanning tunneling microscopy: Imaging of standing electron waves. Phys. Rev. B, 52(19):13796-13798, 1995.

[106] A. Schneider, M. Wenderoth, K. J. Engel, M. A. Rosentreter, A. J. Heinrich, and R. G. U1brich. Local electronic structure at steps on $\mathrm{Au}$ (111) investigated by the thermovoltage in scanning tunneling microscopy. 66(1):S161-S165, 1998.

[107] G. Hörmandinger. Imaging of the $\mathrm{Cu}$ (111) surface state in scanning tunneling microscopy. Phys. Rev. B, 49(19):13897-13905, 1994.

[108] S. Crampin, J. Kröger, H. Jensen, and R. Berndt. Phase coherence length and quantum interference patterns at step edges. Phys. Rev. Lett., 95(2):029701-, 2005.

[109] O. Jeandupeux, L. Bürgi, A. Hirstein, H. Brune, and K. Kern. Thermal damping of quantum interference patterns of surface-state electrons. Phys. Rev. B, 59(24):15926-15934, 1999.

[110] I. Brihuega, P. Mallet, C. Bena, S. Bose, C. Michaelis, L. Vitali, F. Varchon, L. Magaud, K. Kern, and J. Y. Veuillen. Quasiparticle chirality in epitaxial graphene probed at the nanometer scale. Phys. Rev. Lett., 101(20):206802-, 2008.

[111] E. H. Hwang and S. Das Sarma. Screening-induced temperature-dependent transport in twodimensional graphene. Phys. Rev. B, 79(16):165404-, 2009. 
[112] P. Willke. Elektronenstreuung in epitaktisch gewachsenem Graphen auf atomarer Skala. Master's thesis, Universität Göttingen, 2013.

[113] J. Park, G. He, R. M. Feenstra, and An-Ping Li. Atomic-scale mapping of thermoelectric power on graphene: Role of defects and boundaries. Nano Lett., 13(7):3269-3273, 2013.

[114] P. Mallet, F. Varchon, C. Naud, L. Magaud, C. Berger, and J.-Y. Veuillen. Electron states of mono- and bilayer graphene on sic probed by scanning-tunneling microscopy. Phys. Rev. B, 76(4):041403-, 2007.

[115] K. Nagashio, T. Nishimura, K. Kita, and A. Toriumi. Contact resistivity and current flow path at metal/graphene contact. Appl. Phys. Lett., 97(14):143514-3, 2010.

[116] L. Yan, C. Punckt, I. A. Aksay, W. Mertin, and G. Bacher. Local voltage drop in a single functionalized graphene sheet characterized by kelvin probe force microscopy. Nano Lett., 11(9):3543-3549, 2011.

[117] Young-Jun Yu, Yue Zhao, Sunmin Ryu, Louis E. Brus, Kwang S. Kim, and Philip Kim. Tuning the graphene work function by electric field effect. Nano Lett., 9(10):3430-3434, 2009.

[118] T. Druga, M. Wenderoth, F. Lüpke, and R. G. Ulbrich. Graphene-metal contact resistivity on semi-insulating $6 \mathrm{H}-\mathrm{SiC}(0001)$ measured with kelvin probe force microscopy. Appl. Phys. Lett., 103(5):051601-4, 2013.

[119] F. Lüpke. Epitaktisches wachstum von ultradünnen graphitschichten auf der $\mathrm{SiC}(0001)$ Oberfläche: Charakterisierung durch Raster-Kraft- und Raster-Kelvin-Mikroskopie. Master's thesis, IV. Physikalisches Institut Universität Göttingen, 2011.

[120] K1.-D. Katzer, W. Mertin, G. Bacher, A. Jaeger, and K. Streubel. Voltage drop in an (Al[sub $\mathrm{x}$ ] Ga[sub $1-\mathrm{x}]$ )[sub 0.5] $\operatorname{In}[\mathrm{sub} 0.5$ ]p light-emitting diode probed by kelvin probe force microscopy. Appl. Phys. Lett., 89(10):103522-3, 2006.

[121] G. F. Sun, J. F. Jia, Q. K. Xue, and L. Li. Atomic-scale imaging and manipulation of ridges on epitaxial graphene on 6h-sic(0001). Nanotechnology, 20(35):355701-, 2009.

[122] Shuai-Hua Ji, J. B. Hannon, R. M. Tromp, V. Perebeinos, J. Tersoff, and F. M. Ross. Atomicscale transport in epitaxial graphene. Nat Mater, 11(2):114-119, 2012.

[123] M. S. Kang, K. T. Kim, J. U. Lee, and W. H. Jo. Direct exfoliation of graphite using a nonionic polymer surfactant for fabrication of transparent and conductive graphene films. $J$. Mater. Chem. C, 1(9):1870-1875, 2013.

[124] J. Krupka and W. Strupinski. Measurements of the sheet resistance and conductivity of thin epitaxial graphene and Sic films. Appl. Phys. Lett., 96(8):082101-3, 2010.

[125] H. Murrmann and D. Widmann. Current crowding on metal contacts to planar devices. Electron Devices, IEEE Transactions on, 16(12):1022-1024, 1969. 
[126] K.I. Bolotin, K.J. Sikes, Z. Jiang, M. Klima, G. Fudenberg, J. Hone, P. Kim, and H.L. Stormer. Ultrahigh electron mobility in suspended graphene. Solid State Communications, 146(9-10):351-355, 2008.

[127] X. Du, I. Skachko, A. Barker, and E. Y. Andrei. Approaching ballistic transport in suspended graphene. Nat. Nano., 3(8):491-495, 2008.

[128] C. Dimitrakopoulos, A. Grill, T. J. McArdle, Z. Liu, R. Wisnieff, and D. A. Antoniadis. Effect of $\mathrm{SiC}$ wafer miscut angle on the morphology and hall mobility of epitaxially grown graphene. Applied Physics Letters, 98(22):-, 2011.

[129] M. K. Yakes, D. Gunlycke, J. L. Tedesco, P. M. Campbell, R. L. Myers-Ward, C. R. Eddy, D. K. Gaskill, P. E. Sheehan, and A. R. Laracuente. Conductance anisotropy in epitaxial graphene sheets generated by substrate interactions. Nano Lett., 10(5):1559-1562, 2010.

[130] K. W. Clark, X.-G. Zhang, I. V. Vlassiouk, G. He, R. M. Feenstra, and A.-P. Li. Spatially resolved mapping of electrical conductivity across individual domain (grain) boundaries in graphene. ACS Nano, 7(9):7956-7966, 2013.

[131] W. Wang, K. Munakata, M. Rozler, and M. R. Beasley. Local transport measurements at mesoscopic length scales using scanning tunneling potentiometry. Phys. Rev. Lett., 110(23):236802-, 2013.

[132] J. Homoth. Das elektrochemische Potential auf der atomareb Skala: Untersuchung des Ladungstransports eines stromtragenden zweidimensionalen Elektronengases mit Hilfe der Raster-Tunnel-Potentiometrie. PhD thesis, Universität Göttingen, 2008.

[133] John D'Errico. fminsearchbnd. MATLAB Central File Exchange (http://www.mathworks.com/matlabcentral/fileexchange/8277-fminsearchbnd-fminsearchcon), 2012.

[134] F. Giannazzo, I. Deretzis, A. La Magna, F. Roccaforte, and R. Yakimova. Electronic transport at monolayer-bilayer junctions in epitaxial graphene on SiC. Phys. Rev. B, 86(23):235422-, 2012 .

[135] T. Nakanishi, M. Koshino, and T. Ando. Transmission through a boundary between monolayer and bilayer graphene. Phys. Rev. B, 82(12):125428-, 2010.

[136] T. W. Hu, D. Y. Ma, F. Ma, and K. W. Xu. Preferred armchair edges of epitaxial graphene on 6H-SiC(0001) by thermal decomposition. Applied Physics Letters, 101(24):-, 2012.

[137] G. M. Rutter, N. P. Guisinger, J. N. Crain, P. N. First, and J. A. Stroscio. Edge structure of epitaxial graphene islands. Phys. Rev. B, 81(24):245408-, 2010.

[138] J. Jobst, D. Waldmann, F. Speck, R. Hirner, D. K. Maude, T. Seyller, and H. B. Weber. Quantum oscillations and quantum hall effect in epitaxial graphene. Phys. Rev. B, 81(19):195434, 2010 . 
[139] A. J. M. Giesbers, P. Prochazka, and C. F. J. Flipse. Surface phonon scattering in epitaxial graphene on 6H-SiC. Phys. Rev. B, 87(19):195405-, 2013.

[140] J. Jobst, D. Waldmann, F. Speck, R. Hirner, D. K. Maude, T. Seyller, and H. B. Weber. Transport properties of high-quality epitaxial graphene on $6 \mathrm{H}-\mathrm{SiC}(0001)$. Solid State Communications, 151(16):1061-1064, 2011.

[141] J. L. Tedesco, B. L. VanMil, R. L. Myers-Ward, J. M. McCrate, S. A. Kitt, P. M. Campbell, G. G. Jernigan, J. C. Culbertson, C. R. Eddy, Jr., and D. K. Gaskill. Hall effect mobility of epitaxial graphene grown on silicon carbide. Appl. Phys. Lett., 95(12):122102-3, 2009.

[142] V. Perebeinos and Ph. Avouris. Inelastic scattering and current saturation in graphene. Phys. Rev. B, 81(19):195442-, 2010.

[143] S. Lara-Avila, A. Tzalenchuk, S. Kubatkin, R. Yakimova, T. J. Janssen, K. Cedergren, T. Bergsten, and V. Falko. Disordered fermi liquid in epitaxial graphene from quantum transport measurements. Phys. Rev. Lett., 107(16):166602-, 2011.

[144] T. Low, V. Perebeinos, J. Tersoff, and Ph. Avouris. Deformation and scattering in graphene over substrate steps. Phys. Rev. Lett., 108(9):096601-, 2012.

[145] N. Ashcroft and D. Mermin. Solid State Physics. Thomson Learning, Toronto, 1976. 


\section{Abkürzungsverzeichnis}

ADU Analog-Digital-Umsetzer

AFM Rasterkraftmikroskopie, engl. Atomic Force Microscopy

BL zweilagiges Graphen

DAU Digital-Analog-Umsetzer

$\Delta U_{\mathrm{CPD}} \quad$ Kontaktpotentialdifferenz

dI/dU differentielle Leitfähigkeit

e Elementarladung

E Energie

$E_{\mathrm{F}} \quad$ Fermi-Energie

$f(E) \quad$ Besetzungswahrscheinlichkeit der Ladungsträger

$I_{\mathrm{T}} \quad$ Tunnelstrom

$\boldsymbol{j}_{\text {makro }} \quad$ makroskopisch bestimmte Stromdichte

$l_{\mathrm{mfp}} \quad$ mittlere freie Weglänge nach dem Drude-Sommerfeld-Modell

$l_{\varphi} \quad$ Phasenkohärenzlänge

$\lambda_{F} \quad$ Fermiwellenlänge

LDOS lokale Zustandsdichte, engl. Local Density of States

LEED niederenergetische Elektronenbeugung, engl. Low Energy Electron Diffraction

LEEM niederenergetische Elektronenmikroskopie, engl. Low Energy Electron Microscopy

ML einlagiges Graphen

$\mu_{c} \quad$ chemisches Potential

$\mu_{e c} \quad$ elektrochemisches Potential im thermodynamischen Gleichgewicht

$\mu_{e c, S T P} \quad$ über das STP-Verfahren bestimmte ortsabhängige elektrochemische Potential

$n \quad$ Ladungsträgerkonzentration

$\phi \quad$ elektrostatisches Potential

$\phi_{\mathrm{P}} \quad$ lokale Austrittsarbeit der Probe

$\phi_{\mathrm{S}} \quad$ Austrittsarbeit der Spitze

STM Rastertunnelmikroskopie, engl. Scanning Tunneling Microscopy

STP Rastertunnelpotentiometrie, engl. Scanning Tunneling Potentiometry

STS Rastertunnelspektroskopie, engl. Scanning Tunneling Spectroscopy

$T_{\mathrm{P}} \quad$ Probentemperatur 
$T_{\mathrm{S}} \quad$ Spitzentemperatur

$U \quad$ Spannung, $U=\Delta \mu_{e c} / e$

$U_{\text {Bias }} \quad$ Spannungsdifferenz zwischen Spitze und Probe

$U_{\text {ext }} \quad$ Spannung zum Aufprägen eines lateralen Stromes durch die Probenoberfläche

$U_{\text {Kelvin }} \quad$ mit der Raster-Kelvin-Mikroskopie gemessene, räumlich variierende elektrostatische Potentialdifferenz an einer Oberfläche

$U_{\mathrm{Th}} \quad$ Thermospannung

$U_{\text {Trans }} \quad$ Thermospannungsbereinigtes Potentiometrie-Signal bzw. ortsabhängiger Spannungsabfall, hervorgerufen durch einen lateral durch die Probenoberfläche fließenden Strom

UHV Ultra-Hoch-Vakuum 


\section{Danksagung}

An dieser Stelle möchte ich allen Menschen danken, die zum Gelingen dieser Arbeit beigetragen haben und mich während der Promotionszeit begleitet haben.

Zunächst möchte ich Herrn Prof. Dr. Rainer G. Ulbrich für die Ermöglichung dieser Arbeit, seiner Diskussionsbereitschaft und seinen zahlreichen Anregungen danken. Herrn Prof. Dr. Hans Hofsäss möchte ich für die Übernahme der Zweitkorrektur danken.

Ein besonderes Dankeschön richtet sich an Dr. Martin Wenderoth für die Betreuung, die ausdauernde Diskusionsberreitschaft und motivierenden Gespräche während der Erstellung der Dissertation.

Für die gute Zusammenabreit und den Zusammenhalt möchte ich allen ehemaligen und jetzigen Gruppenmitlgiedern der Tunneltruppe danken: Karen Teichmann, Tim Iffländer, Philipp Kloth, Henning Prüser, Karolin Löser, Steffen Rolf-Pissarczyk, Sergej Burbach, Christian Werner und Till Hatje sowie den am Graphenprojekt beteiligten Philip Willke, Felix Lüpke und Philipp Ansorg. Es war immer eine auch außerhalb der Labore und Büros entspannte und unterhaltsame Zeit.

Ein spezielles Dankeschön gebührt Bernhard Spicher, der nicht nur für technische Fragen im Labor immer eine hilfreiche Antwort parat hatte, sondern auch durch seine unnachahmbare Art immer für gute Stimmung gesorgt hat.

Allen weiteren Mitarbeitern des IV. Physikalischen Institutes, insbesondere dem Leiter der Elektronikwerkstatt, Rasit Köster, bei der unermüdlichen Suche nach verlorengegangenen Bits und bei der Optimierung der Messelektronik.

Ganz wichtig war mir die Unterstützung durch meine Familie, besonders meinen Eltern, die mich stets auf meinem Weg begleitet haben.

Zuletzt möchte ich Dir danken Katrin, für die Unterstützung Tag und Nacht im Labor und während des Schreibens sowie der unglaublichen Geduld, die Du während dieser Zeit aufgebracht hast. Danke! 


\section{Lebenslauf}

Name:

Staatsangehörigkeit:

Geburtsdatum:

Geburtsort:

\section{Ausbildung}

seit $04 / 2008$

$12 / 2007$

$10 / 2001$

$06 / 2000$
Thomas Christian Druga

deutsch

13.08.1980

Hameln

Dissertation: „Graphen auf Siliziumcarbid: elektronische Eigenschaften und Ladungstransport"“

Betreuer: Prof. Dr. Rainer G. Ulbrich und Dr. Martin Wenderoth an der Georg August Universität Göttingen

Diplom im Fach Physik

an der Georg August Universität Göttingen

Beginn des Physikstudiums

an der Georg August Universität Göttingen

Allgemeine Hochschulreife

am Viktoria-Luise-Gymnasium Hameln

\section{Publikationen}

1. T. Druga, M. Wenderoth, F. Lüpke and R. G. Ulbrich

Graphene-metal contact resistivity on semi-insulating $6 \mathrm{H}-\mathrm{SiC}(0001)$ measured with Kelvin probe force microscopy,

Appl. Phys. Lett. 103, 051601 (2013).

2. T. Druga, M. Wenderoth, J. Homoth, M. A. Schneider and R. G. Ulbrich A versatile high resolution scanning tunneling potentiometry implementation, Rev. Sci. Instrum. 81, 083704 (2010).

3. J. Homoth, M. Wenderoth, T. Druga, L. Winking, R. G. Ulbrich, C. A. Bobisch, B. Weyers, A. Bannani, E. Zubkov, A. M. Bernhart, M. R. Kaspers and R. Möller Electronic transport on the nanoscale: ballistic transmission and Ohm's law, Nano Lett., 2009, 9 (4), pp 1588-1592.

4. J. Homoth, M. Wenderoth, K. Engel, T. Druga, S. Loth and R. G. Ulbrich Reconstruction of the local density of states in Ag(111) surfaces using scanning tunneling potentiometry,

Phys. Rev. B 76, 193407 (2007). 


\section{Präsentationen}

1. Philipp Ansorg, Thomas Druga, Martin Wenderoth, and Rainer G. Ulbrich Graphene growth by Faco-to-Face method and occurrence of step bunching process Frühjahrstagung der DPG in Regensburg (2013), O 35.85

2. Felix Lüpke, Martin Wenderoth, Thomas Druga, and Rainer G. Ulbrich Investigation of graphene contact resistivity on semi-insulating silicon carbide by Kelvin Probe Force Microscopy Frühjahrstagung der DPG in Regensburg (2013), O 35.88

3. Thomas Druga, Martin Wenderoth, and Rainer G. Ulbrich Local electronic structure of graphene on Si(0001) studied by scanning tunneling microscopy and spectroscopy

Frühjahrstagung der DPG in Dresden (2011), O 60.50

4. Thomas Druga, Martin Wenderoth, and Rainer G. Ulbrich Preparation and characterization study of graphene on $\mathrm{SiC}(0001)$ Frühjahrstagung der DPG in Regensburg (2010), O 41.116

5. Thomas Druga, Martin Wenderoth, Menko A. Schneider, and Rainer G. Ulbrich A Novel Scanning Tunneling Potentiometry Setup with Microvolt Resolution Frühjahrstagung der DPG in Dresden (2009), O 27.21

6. Lars Winking, Martin Wenderoth, Tim Iffländer, Thomas Druga, and Rainer G. Ulbrich Cross-sectional Scanning Tunneling Microscopy across a Metal-Semiconductor Interface Structural and Electronic Properties on the Atomic Scale Frühjahrstagung der DPG in Dresden (2009), O 40.8

7. Thomas Druga., Martin Wenderoth, Jan Homoth, Lars Winking, Rainer G. Ulbrich, Rolf Möller, Bastian Weyers, Armin Bannani, Evgeny Zubkov, Alexander Bernhart, Mark Kaspers Mapping the local transport field in the vicinity of scattering centers with Scanning Tunneling Potentiometry International Conference on Nanoscience + Technology (ICN+T 2008) in Keystone

8. Jan Homoth, Martin Wenderoth, Thomas Druga, Lars Winking, Rainer G. Ulbrich, Mark Kaspers, Alexander Bernhart, Bastian Weyers, Evgeny Zubkov, Rolf Möller and Christian Bobisch

The nanoscale electrochemical potential of a current carrying surface state resolved with Scanning Tunneling Potentiometry

Frühjahrstagung der DPG in Dresden (2008), O 43.28

9. Jan Homoth, Martin Wenderoth, Thomas Druga, Lars. Winking and Rainer G. Ulbrich Imaging of local potentials and thermovoltages with the STM Frühjahrstagung der DPG in Dresden (2006), O29.44 
10. Jan Homoth, Martin Wenderoth, Thomas Druga, Lars Winking and Rainer G. Ulbrich STM-investigations of metal films near the percolation threshold on GaAs(110) DPG-Frühjahrstagung in Dresden (2006), O29.64 